\title{
Ultrafast Control of Magnetism in Ferromagnetic Semiconductors via Photoexcited Transient Carriers
}

\author{
by \\ Ingrid Anda Cotoros \\ B.S. (California Institute of Technology) 2002 \\ M.A. (University of California at Berkeley) 2006 \\ A dissertation submitted in partial satisfaction of the \\ requirements for the degree of \\ Doctor of Philosophy \\ in \\ Physics \\ in the \\ GRADUATE DIVISION \\ of the \\ UNIVERSITY OF CALIFORNIA, BERKELEY
}

Committee in charge:

Professor Daniel Chemla, Co-chair

Professor Dung-Hai Lee, Co-chair

Professor Ramamoorthy Ramesh

Professor Eugene E. Haller

Fall 2008 
Ultrafast Control of Magnetism in Ferromagnetic Semiconductors via Photoexcited Transient Carriers

Copyright 2008

by

Ingrid Anda Cotoros 


\author{
Abstract \\ Ultrafast Control of Magnetism in Ferromagnetic Semiconductors \\ via Photoexcited Transient Carriers \\ by \\ Ingrid Anda Cotoros \\ Doctor of Philosophy in Physics \\ University of California, Berkeley \\ Professor Daniel Chemla, Co-chair \\ Professor Dung-Hai Lee, Co-chair
}

The field of spintronics offers perspectives for seamless integration of coupled and intertunable electrical and magnetic properties in a single device. For integration of the spin degree of freedom with current electronic technology, new semiconductors are needed that show electrically-tunable magnetic properties at room temperature and above. Dilute magnetic semiconductors derived from III-V compounds, like GaMnAs and InMnAs, show coupled and tunable magnetic, transport, and optical properties, due to the fact that their ferromagnetism is hole-mediated. These unconventional materials are ideal systems for manipulating the magnetic order by changing the carrier polarization, population density, and energy band distribution of the complementary subsystem of holes. This is the main theme we cover in this thesis. 
In particular, we develop a unique setup by use of ultraviolet pump, near-infrared probe femtosecond laser pulses, that allows for magneto-optical Kerr effect (MOKE) spectroscopy experiments. We photo-excite transient carriers in our samples, and measure the induced transient magnetization dynamics. One set of experiments performed allowed us to observe for the first time enhancement of the ferromagnetic order in GaMnAs, on an ultrafast time scale of hundreds of picoseconds. The corresponding transient increase of Curie temperature $\left(\mathrm{T}_{C}\right.$, the temperature above which a ferromagnetic material loses its permanent magnetism) of about $1 \mathrm{~K}$ for our experimental conditions is a very promising result for potential spintronics applications, especially since it is seconded by observation of an ultrafast ferromagnetic to paramagnetic phase transition above $T_{C}$. In a different set of experiments, we "write" the magnetization in a particular orientation in the sample plane. Using an ultrafast scheme, we alter the distribution of holes in the system and detect signatures of the particular memory state in the subsequent magnetization dynamics, with unprecedented hundreds of femtosecond detection speed. The femtosecond cooperative magnetic phenomena presented here further our understanding of Mn-hole correlations in III-V dilute magnetic semiconductors, and may well represent universal principles of a large class of carrier-mediated ferromagnetic materials. Thus they offer perspectives for future terahertz $\left(10^{12} \mathrm{~Hz}\right)$ speed "spintronic" functional devices.

Professor Daniel Chemla

Professor Dung-Hai Lee

Dissertation Committee Co-chairs 


\section{To my family}

my home away from home

\section{To Milla and peanut}

light of my life

\&

To Leo

meu amor, o ar que eu respiro 


\section{Contents}

List of Figures $\quad$ iv

List of Tables vii

1 Introduction $\quad 1$

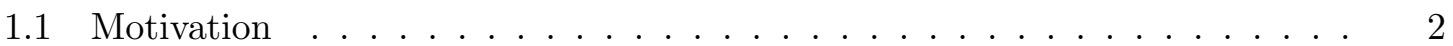

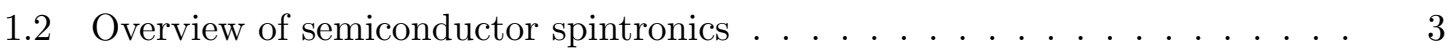

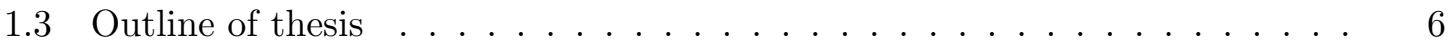

2 Background 9

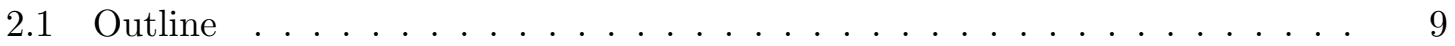

2.2 Dilute magnetic semiconductors . . . . . . . . . . . . . . . . . 10

2.2 .1 III-V Ferromagnetic DMS . . . . . . . . . . . . . . . . . . . 11

2.2 .2 Origin of ferromagnetism in III-V DMS . . . . . . . . . . . . . . . . 13

2.2 .3 (Ga,Mn)As and beyond . . . . . . . . . . . . . . 17

2.2.4 Manipulation of magnetic order via photonic or electric stimuli . . . 19

2.2.5 Memory effects and magnetic memory read-out . . . . . . . . . 23

2.3 Magneto-optical Kerr effect . . . . . . . . . . . . . . . . . . 28

2.4 Femtosecond magneto-optics _. . . . . . . . . . . . . . . 35

2.5 Conclusions . . . . . . . . . . . . . . . . . . . 40

3 Experimental Setup $\quad 41$

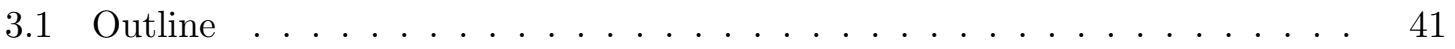

3.2 Samples . . . . . . . . . . . . . . . . . . . . . 42

3.3 The ultrafast laser system . . . . . . . . . . . . . . . . . . . . 48

3.4 Magneto-optical cryostat . . . . . . . . . . . . . . . 52

3.4.1 Cooling the magnet . . . . . . . . . . . . . . 55

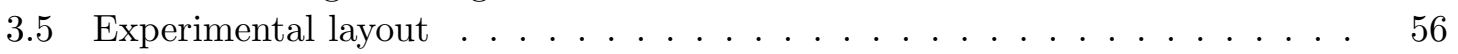

3.6 Time-resolved spectroscopy . . . . . . . . . . . . . . . . . 61

3.6.1 Magneto-optical Kerr effect measurements . . . . . . . . . . . . . 61

3.6.2 Magnetic circular dichroism measurements . . . . . . . . . . . . . 62

3.6.3 Differential reflectivity measurements . . . . . . . . . . . . . 64 
3.7 Experimental parameters and signal analysis $\ldots \ldots \ldots \ldots \ldots$

3.7.1 Excitation density . . . . . . . . . . . . . . . 65

3.7 .2 Signal analysis . . . . . . . . . . . . . . . . 67

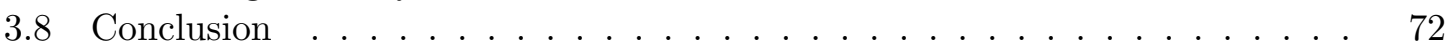

4 Ultrafast Ferromagnetic Phase Enhancement $\quad \mathbf{7 3}$

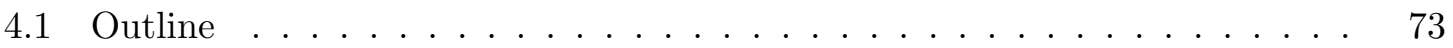

4.2 Magnetization amplitude dynamics in MBE-grown

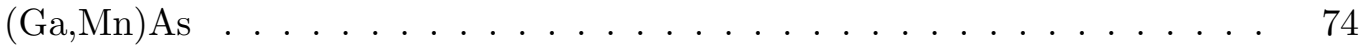

4.2 .1 Charge dynamics . . . . . . . . . . . . . . . . 75

4.2.2 Ferromagnetic phase enhancement . . . . . . . . . . . . 77

4.2.3 Signatures of phase transition . . . . . . . . . . . . . 85

4.2.4 Studies on quasi-2D MBE-grown GaMnAs . . . . . . . . . . . . 89

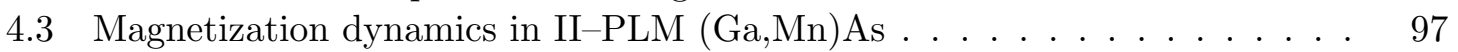

4.3 .1 Charge dynamics . . . . . . . . . . . . . . . . . 98

4.3.2 Giant ferromagnetic phase enhancement . . . . . . . . . . . . . . 99

4.3.3 Discussion . . . . . . . . . . . . . . . . . . . . . . . . . . 103

4.3.4 Ferromagnetic phase enhancement in co-doped samples . . . . . . . 108

4.4 Conclusion . . . . . . . . . . . . . . . . . . . . . 111

5 Coherent Magnetization Dynamics and Magnetic Memory Effects 113

5.1 Outline . . . . . . . . . . . . . . . . . . . 113

5.2 Ultrafast detection of magnetic memory states . . . . . . . . . . . . . 114

5.3 Nanosecond timescale coherent magnetization dynamics . . . . . . . . . . 124

5.3 .1 Magnetic memory . . . . . . . . . . . . . . . . . . 125

5.3.2 Thermal and non-thermal effects in

coherent magnetization dynamics . . . . . . . . . . . . . . 130

5.4 Conclusion . . . . . . . . . . . . . . . . . . . 138

6 Conclusion $\quad 139$

A MOKE: Probe Polarization Rotation vs. Ellipticity Change 142

B Data Acquisition $\quad 144$

$\begin{array}{ll}\text { C Magnetization Dynamics in 3D } & 148\end{array}$

$\begin{array}{ll}\text { Bibliography } & 150\end{array}$ 


\section{List of Figures}

2.1 First report on ferromagnetic $(\mathrm{Ga}, \mathrm{Mn}) \mathrm{As} \ldots \ldots . \ldots . \ldots . . \ldots 13$

2.2 RKKY FM/AFM interaction . . . . . . . . . . . . . . 16

2.3 RKKY interaction vs. distance . . . . . . . . . . . . . . . 16

2.4 Experimental $\mathrm{T}_{C}$ in $(\mathrm{Ga}, \mathrm{Mn})$ As vs. doping concentration . . . . . . . . 18

2.5 PM-FM phase transition induced by photo-generated holes in (In,Mn)As . 21

2.6 Magnetic semiconductor FET schematics . . . . . . . . . . . . . 22

2.7 Reversible, isothermal, electric field operation of magnetic FET . . . . . . . 22

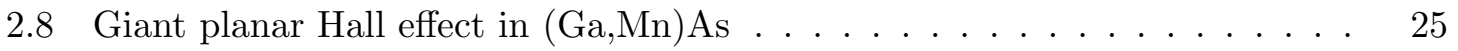

2.9 Giant planar Hall effect in $(\mathrm{Ga}, \mathrm{Mn}) \mathrm{As} \ldots \ldots \ldots . . \ldots . . \ldots 25$

2.10 Giant magnetic linear dichroism in $(\mathrm{Ga}, \mathrm{Mn}) \mathrm{As} \ldots \ldots . . \ldots 27$

2.11 Double transition schematics . . . . . . . . . . . . . . . . 31

2.12 Energy level splitting induced by microscopic interactions . . . . . . . . . . 33

2.13 Three MOKE configurations . . . . . . . . . . . . . . . . 34

2.14 Comparison of pump-induced Kerr rotation and ellipticity in Ni films . . . 37

2.15 Comparison of pump-induced Kerr rotation and ellipticity in $\mathrm{CoPt}_{3} \ldots \ldots$

3.1 GaMnAs samples structures . . . . . . . . . . . . . . . . . 43

3.2 Mn concentration in GaMnAs samples . . . . . . . . . . . . . . . . 43

3.3 LT-MBE sample $\mathrm{M}(\mathrm{T})$ profile . . . . . . . . . . . . . . . . . . 45

3.4 LT-MBE sample hysteresis loop at $5 \mathrm{~K} \ldots \ldots \ldots \ldots \ldots$

3.5 II-PLM sample M(T) profile . . . . . . . . . . . . . . . . 47

3.6 II-PLM sample hysteresis loop at $5 \mathrm{~K} \ldots \ldots \ldots \ldots$. . . . . . . . . . . . . . . . . . 47

3.7 II-PLM sample $\mathrm{R}(\mathrm{T})$ profile . . . . . . . . . . . . . . . . . . . 47

3.8 Schematics of Kerr mode-locking . . . . . . . . . . . . . . . . . . . . 48

3.9 Cavity of Mira 900 laser . . . . . . . . . . . . . . . . . 50

3.10 Typical laser autocorrelation _. . . . . . . . . . . . . . 50

3.11 Schematics of magneto-optical cryostat . . . . . . . . . . . . . 53

3.12 Temperature and magnetic field control . . . . . . . . . . . . . 54

3.13 Ultrafast magnetization dynamics experimental setup . . . . . . . . . . 57

3.14 Beam geometry . . . . . . . . . . . . . . . . . . . 59

3.15 Elliptical beam passing through a QWP . . . . . . . . . . . 63

3.16 Schematics of MOKE signal detection . . . . . . . . . . . . 68 
3.17 Schematics of MCD signal detection . . . . . . . . . . . . . 70

3.18 3D Trajectory of pump-induced magnetization dynamics . . . . . . . . . . 72

4.1 Above $\mathrm{T}_{C}$ differential reflectivity measurements in LT-MBE (Ga,Mn)As: ps

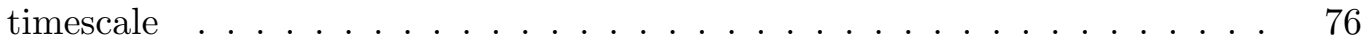

4.2 Above $\mathrm{T}_{C}$ differential reflectivity measurements in LT-MBE (Ga,Mn)As: ns

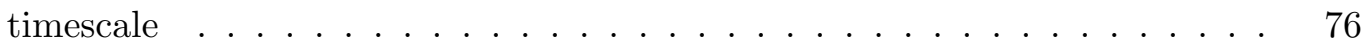

4.3 FM phase enhancement through hole-density-tuning schematics . . . . . . 78

4.4 Temporal traces of photoinduced magnetization changes at different temper-

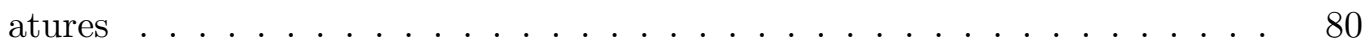

4.5 Temperature dependence of photoinduced demagnetization and magnetization enhancement components . . . . . . . . . . . . . 83

4.6 Qualitative temperature dependence of $\mathrm{Mn}$ and holes spin polarization . . . 84

4.7 Magnetic field dependence of photoinduced demagnetization and magnetization enhancement components $1 \mathrm{~K}$ above $\mathrm{T}_{C} \ldots \ldots \ldots$. . . . . 87

4.8 Build-up time for establishing FM phase vs. magnetic field . . . . . . . . . . 87

4.9 Dynamics signatures of transient para to FM phase transition . . . . . . . 88

4.10 Valence band profile of $\delta$-doped $(\mathrm{Ga}, \mathrm{Mn}) \ldots \ldots \ldots$

4.11 Magnetization versus temperature in quasi-2D (Ga,Mn)As . . . . . . . . 91

4.12 Room temperature $\Delta \mathrm{R} / \mathrm{R}$ in quasi-2D (Ga,Mn)As: ps timescale . . . . . . 92

4.13 Room temperature $\Delta \mathrm{R} / \mathrm{R}$ in quasi-2D (Ga,Mn)As: ns timescale . . . . . . 92

4.14 Comparison of MOKE and MCD signals in quasi-2D (Ga,Mn)As . . . . . 93

4.15 MOKE signal vs. T in quasi-2D (Ga,Mn)As . . . . . . . . . . . . . . 94

$4.16 \Delta \mathrm{R} / \mathrm{R}$ signal vs. $\mathrm{T}$ in quasi-2D $(\mathrm{Ga}, \mathrm{Mn}) \mathrm{As} \ldots \ldots \ldots . \ldots . \ldots . \ldots 9$

4.17 Time slices of dynamic MOKE signal and static properties vs. T . . . . . 96

4.18 Time slices of transient $\Delta \mathrm{R} / \mathrm{R}$ signal and static properties vs. $\mathrm{T} \ldots \ldots$. . . 96

4.19 Room temperature differential reflectivity measurements in II-PLM (Ga,Mn)As 99

4.20 Quenching of induced magnetization at low temperatures in sample C . . . 100

4.21 Temperature dependence of photo-induced magnetization amplitude dynamics in sample C . . . . . . . . . . . . . . . . . . . 102

4.22 Giant FM phase enhancement in sample C . . . . . . . . . . . . . . . 102

4.23 Schematics of top layer phase transition in II-PLM (Ga,Mn)As . . . . . . . 104

4.24 Simulation of $\Delta \mathrm{M} / \mathrm{M}(\mathrm{T})$ enhancement in II-PLM (Ga,Mn)As for $p \propto x \ldots 106$

4.25 Simulation of $\Delta \mathrm{M} / \mathrm{M}(\mathrm{T})$ enhancement in II-PLM (Ga,Mn)As for $p \propto x^{11}$. 107

4.26 Photo-enhanced FM phase in sample D . . . . . . . . . . . . . . . . 109

4.27 Large FM phase enhancement in sample D . . . . . . . . . . . . . . . 109

4.28 FM phase dynamics in sample E . . . . . . . . . . . . . . . . . . 110

5.1 Magnetic memory states in $(\mathrm{Ga}, \mathrm{Mn}) \mathrm{As} \ldots \ldots \ldots \ldots \ldots \ldots$

5.2 Photo-induced four-state magnetic hysteresis . . . . . . . . . . . . 117

5.3 Photo-induced dynamics of "pure" magnetic memory states . . . . . . . . . 119

5.4 Schematics of magnetic field pulse effects . . . . . . . . . . . . . . . 121

5.5 Magnetic memory as observed in ns timescale coherent magnetization dynamics 126

5.6 Magnetic memory in coherent magnetization dynamics hysteresis . . . . . 129

5.7 Thermal effects in coherent magnetization dynamics . . . . . . . . . . 131 
5.8 Phenomenological fit of coherent magnetization dynamics . . . . . . . . . 132

5.9 Thermal effects on magnetization precession frequency . . . . . . . . . . . 133

5.10 Magnetic anisotropy fields as a function of temperature . . . . . . . . . . 134

5.11 Fourier transform of coherent magnetization oscillation at $5 \mathrm{~K} \ldots \ldots$. . . 136

5.12 Magnetic field dependence of M precessional trajectory . . . . . . . . . . . 136

5.13 Magnetic field dependence of magnon frequency . . . . . . . . . . . . 137

A.1 Overlap of the MOKE-MCD signals on the ns timescale . . . . . . . . . 143

A.2 Overlap of the MOKE-MCD signals on the ps timescale . . . . . . . . 143

B.1 LabVIEW testdetector.vi . . . . . . . . . . . . . . . . . . 145

B.2 LabVIEW stagemove.vi . . . . . . . . . . . . . . . . . . 146

B.3 LabVIEW pump-probe PP.vi . . . . . . . . . . . . . . . 147

C.1 Magnon precession in 3D . . . . . . . . . . . . . . . . 149 


\section{List of Tables}

3.1 Properties of $\mathrm{Ga}_{1-x} \mathrm{Mn}_{x}$ As samples investigated in this work . . . . . . . 44

3.2 Essential equipment list . . . . . . . . . . . . . . . . 60 


\section{Acknowledgments}

"In science, the proof of the pudding is in the eating, that's the only way to counter skepticism" Daniel Chemla. I start by thanking my thesis adviser for giving me the opportunity to work in his group, and for guiding me along the way. He was an incredible role-model to me, and always supported me in my research. For that, I am very grateful. Most of all, he taught me courage, and how to persevere against all odds.

A very special THANK YOU goes to Jigang Wang, a brilliant experimentalist with an inquisitive mind, now professor at Iowa State University. As a postdoc in our group, Jigang was a dedicated mentor to me and a dear friend throughout my years at Cal. He taught me everything I know about ultrafast MOKE spectroscopy, and guided me in my research, data acquisition and interpretation, conference presentations, and my writing. I am sure that our friendship and collaboration will continue throughout our lives.

During the course of my graduate research, I had the good fortune to work with a talented and hard-working bunch of young scientists. Keshav Dani was the one to "recruit" me to the group, and to teach me basic optics techniques and operation of the magnet. I had many inspiring conversations, fun hours, and life lessons from Rupert Huber, my personal model of ethics, Ben Schmid, who taught me squash, and Nils Nielsen, the ever pragmatic source of advice. I wish them all the best of luck.

I am grateful to my collaborators, Profs. Jacek Furdyna and Xinyu Liu at University of Notre Dame, for providing me with the best quality GaMnAs samples, and generously sharing their extended experience; to Prof. Ilias Perakis at University of Crete, in Greece, for his ever innovative contribution to our interpretations of data; to Prof. Oscar Dubon and 
to Peter Stone at our very own UC Berkeley, for their openhanded partnership on studying ion-implanted, pulsed-laser melted GaMnAs. I am also thankful to Wladek Walukiewicz at LBNL and Lukasz Cywinski at University of Maryland for our very stimulating discussions on magnetization dynamics in dilute magnetic semiconductors.

Several people showed me great kindness and encouragement to finish my Ph.D. In particular, I thank Robert Kaindl and Prof. Eugene Haller for providing me much needed feedback in writing my thesis. I also thank Profs. Dung-Hai Lee, Ramamoorthy Ramesh, and Marjorie Shapiro for serving on my qual and thesis committees.

I appreciate the help of Anne Takizawa, a tremendous asset to the Physics department, and in my eyes, one of its vital ingredients. I particularly thank Profs. Eugene Commins, Hitoshi Murayama, Marvin Cohen, and Eugene Haller, who through their incredible dedication as educators, made my classroom experience at Cal unique, and one that I will tell my grandchildren about.

Last but not least, I would like to thank my friends and my family. They were an incredible source of support, encouragement, and inspiration. My friend and my mentor, Ab, thank you for always believing in me, and for teaching me the most important life lessons. My family, thank you for your sacrifices, for allowing me to cross the globe to follow my passion, and for your ever present love. And my own small family, Leo and Milla, thank you for your purest love, patience, support and incredible joy you bring to my life.

This work was supported by the Director, Office of Science, Office of Basic Energy Sciences, of the U.S. Department of Energy under Contract No. DE-AC02-05CH11231. 


\section{Chapter 1}

\section{Introduction}

Those who control materials will control technology Tadahiro Sekimoto

Information-processing semiconductor-based devices are one of the most sophisticated, most complex, most high-performance, but also most expensive technologies nowadays. Switching within information-processing devices is performed by the controlled motion of small pools of charge, while information storage and retrieval in magnetic storage devices is performed by reorienting magnetic domains. New fabrication lines with costs approaching $\$ 3.5$ billion, with $25 \%$ of the tools obsolete and needing replacement every three years, put a tremendous pressure on this technology for progress. But for how long can this progress be fueled by the miniaturization of transistors in microprocessors and of memory cells in magnetic and optical discs? And how will the needs for reduced power consumption and integration with modern multifunctional/ bio devices be addressed? Semiconductor spintronics offers an innovative direction towards the development of hybrid devices that could perform logic, communications and storage operations within the same technology. 


\subsection{Motivation}

The spin degree of freedom of elementary particles, mostly ignored in the development of charge-based electronic devices, has come into attention in the past decade as basis for potential future spintronic devices. Exploring and understanding the spin states of magnetic semiconductors and their quantum-confined structures in particular has gathered tremendous interest, fueled by the perspectives of seamless integration of coupled and inter-tunable electrical and magnetic properties in a single device. In order to integrate the spin degree of freedom with current electronic technology, new semiconductors are needed that show electrically-tunable magnetic properties at room temperature and above.

One of the most remarkable progress in the field of semiconductor spintronics was the discovery of carrier-mediated, high Curie temperature ferromagnetic (FM) order in (III,Mn) V dilute magnetic semiconductors, like GaMnAs, or InMnAs. These materials show coupled and tunable magnetic, transport, and optical properties, due to hole-mediated ferromagnetism. The manganese $(\mathrm{Mn})$ ions replacing the III $^{r d}$ group atoms provide local magnetic moments, as well as acceptors (holes) to the system. These valence band holes antiferromagnetically couple to the $\mathrm{Mn}$ ions, ensuring the long range order in the system. These unconventional materials are ideal systems in which to control the magnetic order by manipulating the complementary subsystem of holes, in particular its population density, spin polarization, and energy distribution. 


\subsection{Overview of semiconductor spintronics}

For the past few decades, Moore's law has held true: miniaturization has lead to an exponential increase in the quantity of information that can be processed, stored, and transmitted per unit area of microprocessor, memory, and fiberglass, respectively. Nowadays, a modern integrated circuit contains $\approx 1,000,000,000$ transistors, each smaller than $100 \mathrm{~nm}$. Nanotechnology has taken a pivotal role since the $100 \mathrm{~nm}$ lengthscale per transistor threshold has been crossed at the beginning of this century. As transistor manufacturing matures, the price per unit continues to decrease. However, as the size has reached the nanoscale, fundamental physical limitations due to quantum effects are beginning to increasingly affect the basic functionality of transistors and other circuit elements. Thus, there is prevalent sense that in the near future a disruptive innovation is needed to continue the ever increasing trends in data processing, storing, encoding, and transmission.

Among the many future directions into which nanotechnology can evolve, the field of spintronics plays a major role. Spintronics is an emerging technology aiming to understand spin transport electronics and electron spin phenomena. Its goal is to exploit the intrinsic spin degree of freedom (and its associated magnetic moment) of electron in addition to or in place of the charge (or orbital) degree of freedom to create novel multifunctional solid state devices.

An ambitious goal of spintronics is to create magnetoresistive random access memory (MRAM), a device that would combine the advantages of non-volatile magnetic memory (i.e., using ferromagnetic storage elements) and dynamic random access memory (DRAM, based on accumulated electric charge, which requires frequent refreshing). This requires 
novel methods to magnetize memory cells and read back the direction of the written magnetization without use of mechanical systems. Another needed innovation is the ability to control magnetization isothermally, by means of light or electric field: in contrast, large fractions of the energy spent by current devices goes to controlling magnetization (storing data) via magnetic fields generated indirectly by electric currents.

Another goal of spintronics is to create hardware for quantum information science, by applying modern schemes for performing computations using the superposition and entanglement of quantum states rather than the binary on/off system used in electronics. Experiments conducted by David Awschalom's group in Santa Barbara have shown that spin degrees of freedom are of particular importance as they maintain their phase coherence significantly longer than the orbital (charge) degrees of freedom [80]. Therefore, the spin of electrons is a much more suitable choice than the charge of electrons for spintronic devices. Spin nanostructures might thus alter the basic principles not only in the design of electronic elements, but also in the very computer architecture that has been in use for half a century.

A diverse range of materials are used in today's research on spin electronics. Most advanced studies are on magnetic multilayers, systems which exhibit giant magnetoresistance (GMR) [5, 10]. The Nobel prize in physics was awarded to Albert Fert and Peter Grünberg for the discovery of GMR. GMR comes from spin-dependent scattering at adjacent interfaces between non-magnetic and magnetic metals, which changes when the magnetic field aligns magnetization of particular layers [13]. This effect has been successfully applied and is exploited commercially by manufacturers of read/ write heads of high-density hard-disks drives. More recent work has focused on spin-dependent tunneling via an oxide 
film. In the case of crystalline $\mathrm{MgO}$ sandwiched between contacts of amorphous $\mathrm{Fe}-\mathrm{Co}-\mathrm{B}$ layers, remarkably, the difference between tunneling resistance for anti-parallel vs. parallel orientation of the magnetization (tunnel magnetoresistance, TMR) reaches a factor of three at $300 \mathrm{~K}[65,105,38]$. Moreover, the magnetization direction can be switched by an electric current, at current densities below $10^{6} \mathrm{~A} \mathrm{~cm}^{-2}$ [33]. This opens the door to direct magnetization writing via current pulses.

Last but not least, such structures can be used for injecting highly polarized spin currents into semiconductors. Spin transistors, which are devices composed of two layers of ferromagnetic conductors separated by a non-magnetic material, rely on the injection of spin-polarized carriers in the non-magnetic layer. The electrical conductivity then depends on the relative direction of the magnetization vectors in the ferromagnetic layers. Spin transistors offer an energy-conserving and fast switching device alternative to regular transistors, as they allow current to be controlled without changing the carrier concentration.

In the course of the years, semiconductor spintronics has evolved into a rather broad research field. For instance, progress in fabrication and studies of spin quantum gates of double quantum dots has been described by van der Wiel et al. [89]. A comprehensive survey on spin-orbit effects and the present status of spin semiconductor transistors has been written by Zutic, Fabian, and Das Sarma [37]. Finally, Jungwirth et al. have reviewed various aspects of the theory of $(\mathrm{Ga}, \mathrm{Mn}) \mathrm{As}$ and related materials [40]. Furthermore, more comprehensive reviews of the entire semiconductor spintronics field are also available $[100$, $62,4]$. 


\subsection{Outline of thesis}

We began this thesis by providing a motivation for our focus on ferromagnetic semiconductors as basis of future spintronic devices. This study merges the physics of collective ordering and dynamics of spin states in ferromagnetic semiconductors with ultrafast time-resolved spectroscopy techniques as powerful tools to probe spin relaxation of non-equilibrium photoexcited carriers, transient dynamics of ferromagnetic order, and photo-induced dynamic phase transitions. These results have the potential to open avenues for integration of the spin degree of freedom into current electronic applications, in particular terahertz speed spintronic devices and multi-functional systems.

Chapter 2 provides the background material for understanding the experiments. The previous discovery of carrier-mediated ferromagnetic order in (III,Mn)V semiconductors, one of the most important advances in semiconductor spintronics, is discussed here in the context of dilute magnetic semiconductors. Basic concepts for understanding this charge-mediated ferromagnetic order, and relevant (static) experiments on the electrical/photo control of ferromagnetism in (III,Mn)Vs are presented next. These experiments have sparked intense interest in exploring the novel functionalities arising from the interplay of charges, spins, and photons in semiconductors. We end the chapter presenting the magneto-optical Kerr effects, and reviewing important time-domain experiments exploring the dynamic magneto-optical processes in GaMnAs.

In chapter 3, we outline the sampled studied, the experimental apparatus, and the different techniques that allow us to study photoexcited carrier spin relaxation dynamics, ferromagnetic order dynamics, and ultrafast phase transitions. In particular, we describe in 
detail the experimental apparatus and methods, including formulae that allow us to extract magnetization $(\mathrm{M})$ or other relevant physical quantities out of the raw data.

Experimental results are presented in chapters 4 and 5. Chapter 4, in particular, focuses on the ultrafast enhancement of the ferromagnetic order in both MBE-grown GaMnAs, and ion-implanted GaMnAs. In MBE-grown GaMnAs, the enhancement of the ferromagnetic order comes as a consequence of photoexciting extra carriers that participate in mediating ferromagnetism and thus strengthening this order parameter. As a consequence, a transient phase transition is expected to occur right above $\mathrm{T}_{C}$ and, indeed, signatures of such a transition are observed. In ion-implanted GaMnAs, the enhancement, while fundamentally the same at a microscopic level, exhibits a different macroscopic experimental signature, due to the varying concentration of Mn from the sample surface inwards. As a consequence, the enhancement of ferromagnetic order is drastically larger, but disappears at a specific temperature below $\mathrm{T}_{C}$. We can explain this behavior by phase transitions that occur in the first few $\mathrm{nm}$ at the sample surface, i.e., in layers that are not in a ferromagnetic state before photoexcitation.

We discuss the theoretical framework used to explain various dynamics observed in GaMnAs at photoexcitation. This includes the initial demagnetization, which is due to the photoexcitation of spin-unpolarized hot carriers in high momentum states. These carriers, as it turns out, experience effective spin scattering with the magnetic moments, inducing a pulse-limited demagnetization effect. On the longer time scale, phonon-scattering induced demagnetization is also observed. On a timescale of several 100 ps, a build-up of the magnetic order is observed, due to the excess photoexcited carriers that no participate in 
mediating ferromagnetism. A stronger ferromagnetic order translates into increased $T_{C}$. In ion-implanted GaMnAs, we find that a refinement of this model is needed to account for the spatially-varying concentration of Mn atoms inwards from the surface, and consequently, the variation of hole concentration and $\mathrm{T}_{C}$.

In chapter 5 we present some interesting effects of magnetic memory states in GaMnAs on the magnetization dynamics in this system. Based on the observed behavior, we propose an ultrafast detection scheme that allows for read-out of magnetic memory states with sub-picosecond (ps) speed. We also present the coherent effects of magnetic memory states on the magnetization dynamics on a longer, nanosecond (ns) timescale. We find that the dynamics on both sub-ps and ns timescales can be explained by a combination of non-thermal, photoexcited carriers effects, and a thermal, phonon-assisted mechanism. We attribute the non-thermal effects to a pulse-limited magnetic field pulse which arises from the photoexcitation and distribution of carriers in high momentum states, which modifies the existing magnetic anisotropy fields. The thermal effects can be explained in turn to be due to a phonon-assisted modification of magnetic anisotropy fields, turning on a magnon precession.

The last chapter presents a summary of this work, emphasizing the critical role of magneto-optical spectroscopy for understanding (III,Mn)V semiconductors. We end with describing a few new leads for interesting work that can build on the results presented in this thesis and give an overview of future directions in the field of spintronics. 


\section{Chapter 2}

\section{Background}

\subsection{Outline}

We talked about the "why". In this chapter, we will present the "how": how to make semiconductors magnetic, and how to control magnetism. We will present the experiments that have captured the intense interest of the scientific community in exploring the novel functionalities arising from the interplay of charges, spins, and photons. In particular, we will discuss the manipulation of magnetic order in ferromagnetic semiconductors via photonic or electric stimuli, and the giant magneto-transport and magneto-optical effects in these systems, which bring about rich memory effects and allow for super-resolution magnetic state read-out.

Background material for understanding our samples and experiments, a review of properties of dilute magnetic semiconductors, with particular focus on III-Mn-V materials, and concepts we use in probing magnetization dynamics at ultrafast timescales (i.e., magneto-optical Kerr effect and femtosecond magneto-optics) will also be presented. 


\subsection{Dilute magnetic semiconductors}

In today's modern technology, information is stored in localized magnetic moments, processed by charge currents, and transmitted using light. To enhance the performance of devices, both the charge and spin of electrons ought to be used. There are two directions that immediately come into mind on how one should go about incorporating both electronic properties into spintronics devices: one could inject spin carriers in semiconductors, or directly use magnetic semiconductor materials.

However, it is difficult to interconnect first-generation spintronic devices, based on alloys of magnetic metals (nickel, cobalt, iron), with current-generation semiconductor technology. This is mainly due to crystal structure incompatibilities between metallic alloys and semiconductors, such as lattice mismatch, difference in conductivity, and low spin injection ratio.

On the other hand, the semiconductor industry materials of choice for devices and integrated circuits, Si and GaAs, do not naturally contain magnetic ions and are nonmagnetic. Their magnetic Landé-g factors are so small that they would require enormous magnetic fields to differentiate between, and thus put to use the two possible electron spin orientations. Even though some natural ferromagnetic semiconductors do exist (such as

europium chalcogenides and semiconducting spinels that have a periodic array of magnetic elements), such materials are very hard to grow, and their crystal structure is also quite different from that of Si or GaAs.

These problems can all be significantly reduced by exploiting one of the most useful properties of semiconductors, the ability to accept dopants that change their properties. 
Usually n or p-type, doping can be extended to include magnetic dopants, such as magnetic transition metal ions (mainly Mn), into semiconductors such as GaAs and CdTe [24, 2]. In these materials, dilute magnetic semiconductors (DMS), a sizable portion of lattice sites has been substituted by elements such as $\mathrm{Mn}$, giving rise to localized magnetic moments in the semiconductor matrix (i.e., (Ga,Mn)As, $(\mathrm{Zn}, \mathrm{Cr}) \mathrm{Se},(\mathrm{Pb}, \mathrm{Eu}) \mathrm{Te})$. Of key importance to DMS is a spin-dependent coupling between localized spin states and those in the valence and conduction bands. Because of this coupling, holes in DMS mediate a ferromagnetic interaction between the localized spins, which makes it possible to control magnetism by controlling the carrier density in semiconductors.

II-VI DMS based on CdTe and ZnSe, in which the valence of the cations matches that of the common magnetic dopant ions such as Mn have been the focus of intense study [27]. Though these materials and their heterostructures are relatively easy to prepare both in bulk and in thin epitaxial layers, and despite some progress obtained in doping them $[78,6]$, they have traditionally been difficult to dope (e.g., nitrogen doping), which makes them less suitable for applications. Also, the main magnetic interaction in II-Mn-VI materials is the antiferromagnetic exchange interaction between Mn spins, which results in paramagnetic $(\mathrm{PM})$, antiferromagnetic $(\mathrm{AFM})$, or spinglass behavior. Ferromagnetism in II-VI, Mndoped semiconductors was discovered only recently in modulation-doped CdMnTe quantum wells, and only at very low temperatures $[32,20]$.

\subsubsection{III-V Ferromagnetic DMS}

An important class of DMS are the magnetic semiconductors derived from III-V compounds. Since III-V semiconductors are already used in a variety of electronic and 
optoelectronic devices (i.e., microwave transistors in cell phones, semiconductor lasers for CDs), the introduction of III-V based DMS semiconductors opens up the possibility of using a variety of magnetic phenomena to expand on the functionalities of already established optical and electrical devices.

The low solubility of magnetic elements (such as Mn) in the III-V compounds has been the biggest obstacle to obtain the high dopant concentration necessary to make these semiconductors magnetic. Since one expects magnetic effects roughly proportional to the concentration of magnetic ions, one doesn't expect a major change in properties at the solubility limit of magnetic impurities, usually on the order of $10^{18} \mathrm{~cm}^{-3}$ or less.

A breakthrough was made with the discovery that low temperature molecular beam epitaxy (MBE) allowed the growth of III-V DMS with magnetic ion concentrations far above the solubility limit. When a high concentration of magnetic elements is introduced in excess of the solubility limit, a second phase begins to form if conditions are near equilibrium. However, if the crystal is grown by MBE at low temperatures, the thermal energy is not enough to allow the formation of this second phase, yet it allows epitaxial growth of a single-crystal alloy. The effort to grow new III-V based DMS by low-temperature MBE was rewarded with successful epitaxial growth of uniform (In,Mn)As films on GaAs substrates by Munekata in 1989 [57], where partial ferromagnetic order was found, and ferromagnetic (Ga,Mn)As films by Ohno in 1996 [63] (Fig. 2.1). Moreover, dependencies of lattice constants versus Mn composition in (In,Mn)As and (Ga,Mn)As films extrapolate to the same value (i.e., the lattice constant of hypothetical zincblende MnAs), building a strong argument that all of the Mn atoms are incorporated in the zincblende alloy in these 
compounds.

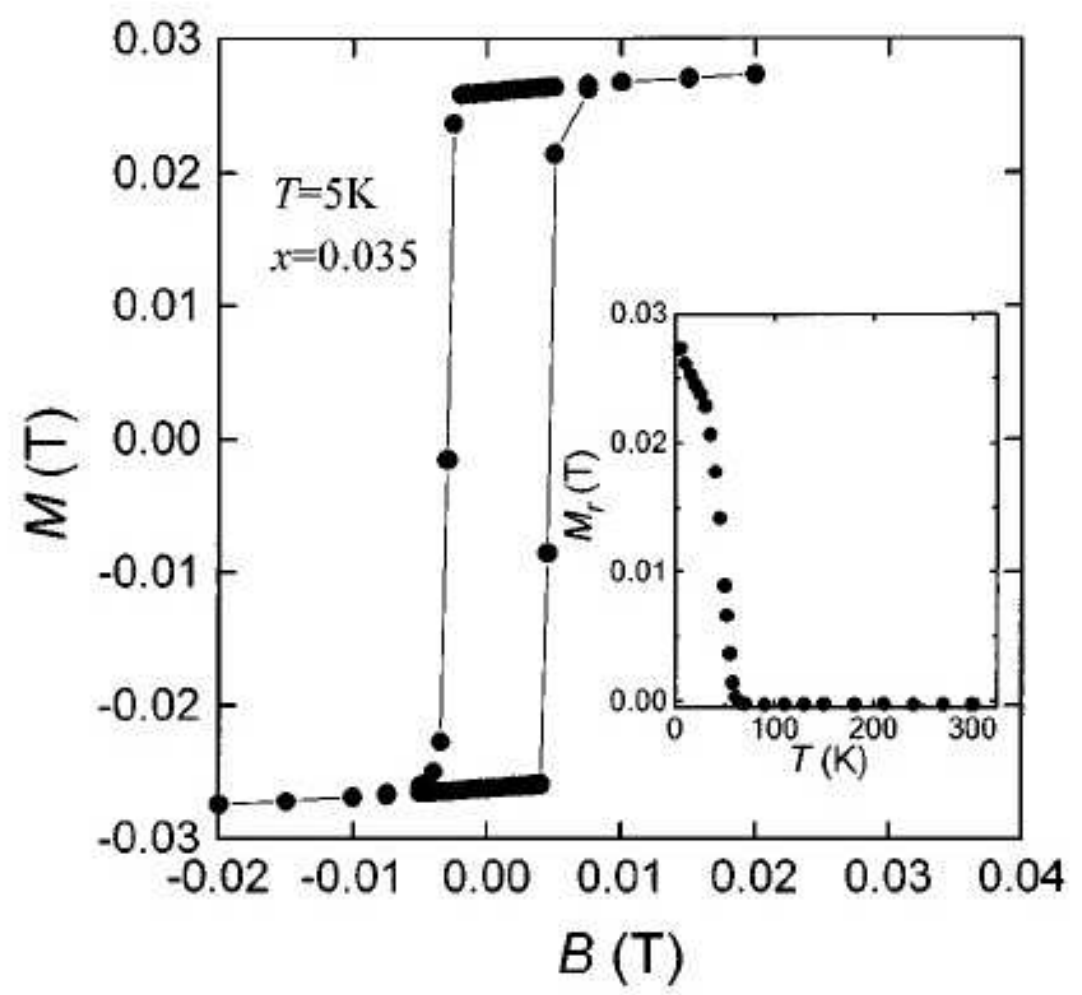

Figure 2.1: From ref. [63]: Magnetic field dependence of magnetization $\mathrm{M}$ at $5 \mathrm{~K}$ for a (Ga,Mn)As film with $\mathrm{x}=3.5 \% \mathrm{Mn}$ content. The field was applied parallel to the sample surface. Inset shows the temperature dependence of the remnant $\mathrm{M}$ of the same sample.

\subsubsection{Origin of ferromagnetism in III-V DMS}

The magnetic interaction between Mn spins has been shown to be antiferromagnetic in n-type (In,Mn)As [91] and in fully carrier compensated (Ga,Mn)As [73]. This implies that the ferromagnetic interaction is hole induced. It is now widely accepted that the ferromagnetism in $(\mathrm{Ga}, \mathrm{Mn})$ As arises from the hole-mediated interaction between local magnetic moments of the $\mathrm{Mn}_{G a}$ acceptors, but details of the mechanism are still poorly understood and important parameters remain unknown [40]. The double-exchange model, 
involving the interaction $-J \overrightarrow{S_{i}} \overrightarrow{\sigma_{i}}$ between the magnetic impurity spin $\vec{S}_{i}$ and the electron spin $\overrightarrow{\sigma_{i}}$ has been the starting point in nearly all theoretical studies. In the mean-field picture (Zener model), long-range ferromagnetic interaction between the $\mathrm{S}=5 / 2 \mathrm{Mn}^{2+}$ ions is mediated by a uniform itinerant-carrier spin polarization caused by an effective uniform magnetic field, resulting from a site averaging of local impurity fields. An alternative mechanism for the ferromagnetic coupling between impurity-spins involves the hole-mediated Ruderman-Kittel-Kasuya-Yosida (RKKY) interaction. This mean-field theory has been proposed to explain the ferromagnetism in $(\mathrm{Ga}, \mathrm{Mn}) \mathrm{As}$ [21], since the RKKY framework was shown to successfully explain the carrier-induced ferromagnetism in a IV-VI compound $(\mathrm{Pb}, \mathrm{Sn}, \mathrm{Mn}) \mathrm{Te}[84]$.

The RKKY interaction was originally proposed by M.A. Ruderman and Charles Kittel of U. C. Berkeley [69] as a means of explaining unusually broad nuclear spin resonance lines that had been observed in natural metallic silver. It was later expanded by Tadao Kasuya of Nagoya University [42] from nuclear spins to localized inner d-electron spins, and by Kei Yosida of U. C. Berkeley [102] to give a Hamiltonian that describes all of (d-electron spin)-(d-electron spin), (nuclear spin)-(nuclear spin), and (d-electron spin)(nuclear spin) interactions. The theory uses second-order perturbation theory to describe an indirect exchange coupling whereby the inner d-electron spins of one atom couple to a conduction electron via the exchange interaction, and this conduction electron then interacts with another d-electron spin, thus creating a correlation energy between the two spins. The theory is based on Bloch wavefunctions, and is therefore only applicable to crystalline systems. 
Assuming a spin dependent energy interaction of the form $H=-J \vec{\sigma} \vec{S}_{i} \delta(\mathbf{r}-$ $\mathbf{R}_{\mathbf{i}}$ ), a general susceptibility can be calculated [106], and evaluated to a simple analytic function with a second-derivative susceptibility at $q=2 k_{F}$. A Fourier transform (to the spatial distribution of magnetization in the neighborhood of the localized moment) shows the singularity to give rise to an oscillatory term, falling off as $1 / r^{3}$ at larger distances. This translates into a FM/ AFM interaction oscillation with distance between the two spins (Figs. 2.2 and 2.3). This prediction of RKKY was successfully applied to explain coupling between thin layers of magnetic materials separated by a non-magnetic spacer, found to oscillate between FM and AFM as a function of spacer size, and led to the discovery of GMR [28].

With the hole concentration determined from magneto-transport measurements, the Curie temperature $\mathrm{T}_{C}$ can be calculated from the exchange constant. Although the results are slightly dependent on the cut-off length of the RKKY interaction, the calculated $\mathrm{T}_{C}$ shows good agreement with the experimentally determined $\mathrm{T}_{C}[55]$. The understanding of the ferromagnetism of $(\mathrm{Ga}, \mathrm{Mn}) \mathrm{As}$ is, however, still not adequate. For example, the behavior of the critical scattering may be qualitatively different when the spin-spin interaction is of long range as opposed to the short range interaction [32]. Also, theoretical work based on first principles calculations found unusual crystallographic directional dependence of the exchange energy that brings into question the RKKY-based models [54]. Another experimental result on hole polarizations using hot-photoluminescence spectra found that most of the valence band holes are not free, but bound to Mn acceptors or confined to an impurity band, thus making them unable to support the RKKY-like exchange interaction via degenerate or weakly localized holes [72]. 


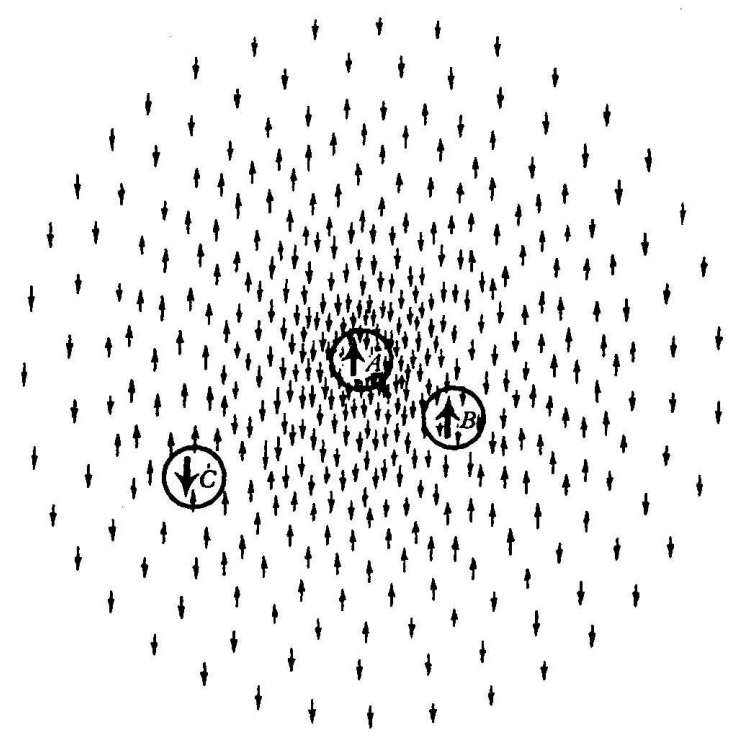

Figure 2.2: From ref. [106]: Oscillations of spin polarization of the s-electrons (conduction) about the magnetic impurity A lead to a ferromagnetic interaction with the neighboring impurity B, but may favor antiferromagnetism at a greater distance, C.

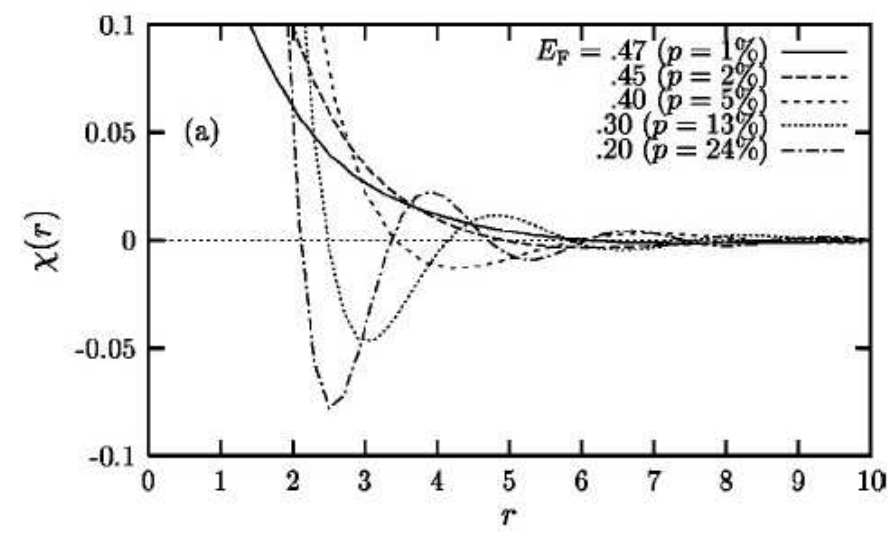

Figure 2.3: From ref. [79]: The behavior of the magnetic susceptibility $\chi(r)$ with $r$, the average $\mathrm{Mn}-\mathrm{Mn}$ separation, for different hole doping concentrations $\mathrm{p}$. 
There are still issues remaining to be studied such as to what extent the "pure" RKKY interaction is applicable to $(\mathrm{Ga}, \mathrm{Mn})$ As. However, extensions to RKKY that take into consideration the spatial variations of the impurity-induced carrier spin polarization beyond linear theory [79], or dynamical mean-field theory that includes local charge and spin fluctuations [15] are still the workhorse in predicting magnetic behavior and transition temperatures for (Ga,Mn)As.

\subsection{3 (Ga,Mn)As and beyond}

The key difference between (Ga,Mn)As and II-VI DMS is that in (Ga,Mn)As, the Mn substituting for the trivalent cation Ga is simultaneously an acceptor and a source of magnetic moments, while in II-VI it does not act as an acceptor. A small amount of Mn ions incorporated into GaAs provides itinerant holes that modify the valence-band structure of the host material and mediate the coupling between the localized spins, giving rise to ferromagnetism. Today, strongly ferromagnetic GaMnAs films are grown by MBE at low temperatures with concentrations up to $9 \%$ [104] and despite steady progress, $\mathrm{T}_{C}$ of only up to $173 \mathrm{~K}$ [41]. Theoretical critical-temperature calculations based on the kinetic-exchange model predict room-temperature ferromagnetism in (Ga,Mn)As with 10\% substitutional Mn content [8]. However, the current $\mathrm{T}_{C}$ record cannot be broken until DMS material with a higher concentration of substitutional Mn ions can be grown (Fig. 2.4). The position of the Fermi level controls the number of $\mathrm{Mn}_{G a}$ ions and the hole concentration, thus affecting the Curie temperature. Photoemission studies showed that the relative position of the Fermi level is found to be concentration dependent. For concentrations around $3.5 \%-5 \%$ it is located very close to the valence-band maximum, while for concentrations outside this 


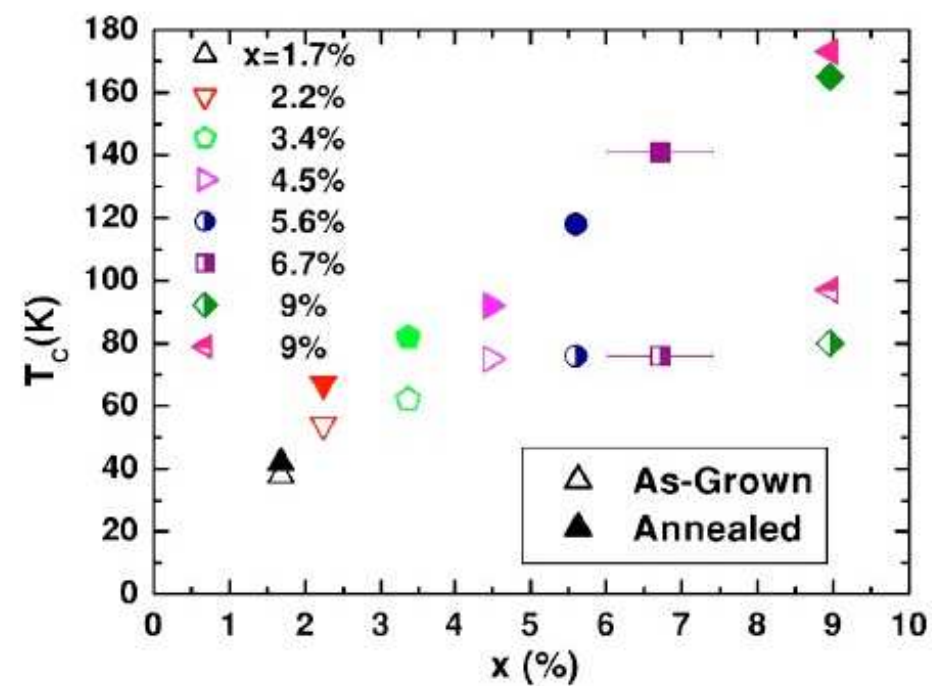

Figure 2.4: From ref. [41]: Experimental Curie temperature vs. total Mn doping. Open symbols correspond to as-grown samples, half-open symbols to as-grown samples with large charge compensation, and filled symbols to annealed samples. Error bars are shown only for the $\mathrm{x}=6.7 \%$ sample.

range, higher as well as lower, the Fermi level is found to be pinned at about $0.15 \mathrm{eV}$ higher energy [1].

Low-temperature MBE grown $(\mathrm{Ga}, \mathrm{Mn})$ As films display several types of intrinsic defects. Mn ions can substitute $\mathrm{Ga}$ to form substitutional $\mathrm{Mn}_{G a}$, enter interstitial positions to form $\mathrm{Mn}_{I}$, or reside at random sites to form $\mathrm{Mn}_{A s}$ clusters $[104,23]$. The $\mathrm{Mn}_{G a}$ ions act as acceptors with a level at about $110 \mathrm{meV}$ above the top of the valence band, each binding a shallow hole $[23,49]$. Self-compensation occurs when the interstitial $\mathrm{Mn}_{I}$ ions reduce the carrier density and the number of active $\mathrm{Mn}_{G a}$ ions by forming antiferromagnetic $\mathrm{Mn}_{\mathrm{Ga}}{ }^{-}$ $\operatorname{Mn}_{I}$ pairs $[104,23,82,11]$. Recent experiments which show that the Curie temperature increases with low-temperature annealing (around 555-600 K) can be explained by the creaking of the relatively weak $\mathrm{Mn}_{I}-\mathrm{Mn}_{G a}$ pairs. In this case, the $\mathrm{Mn}_{I}$ ions segregate to the film surface and form oxides $[104,23,56]$. Other than neutral $\mathrm{Mn}_{G a}$ acceptors, ionized 
$\mathrm{Mn}_{G a}(\mathrm{Mn} 3+)$ ions also exist [39]. Another common defect in (Ga,Mn)As is the As antisite, which acts as a deep donor, thus removing As-antisites could increase the magnetic moment and Curie temperature [46]. A study using positron annihilation spectroscopy and infrared absorption measurements showed that the As-antisite concentration increases with the Mn doping concentration [88].

The ability to tune the ferromagnetism of $(\mathrm{Ga}, \mathrm{Mn}) \mathrm{As}$ by varying the Mn concentration can already be exploited in quantum information hardware, such as spin sources and detectors of single spins. Although it is a big challenge to realize room temperature ferromagnetism in $(\mathrm{Ga}, \mathrm{Mn}) \mathrm{As}$, the system is still very useful in demonstrating spintronics applications concepts. One can apply the knowledge obtained from this material to new ferromagnetic semiconductor materials that show considerable spontaneous magnetization and coupling between the semiconductor and magnetic degrees of freedom at room temperature. The Mn-doped wide-bandgap nitrides (i.e., (Ga,Mn)N) and oxides (i.e., p-(Zn,Mn)O) are particularly promising. ( $\mathrm{Zn}, \mathrm{Mn}) \mathrm{O}$ is generally antiferromagnetic, but doping with other transition metals such as $\mathrm{V}, \mathrm{Cr}, \mathrm{Fe}$, Co and Ni can result in half-metallic ferromagnetism [18]. Considerable research in semiconductors resulted in room temperature ferromagnetism in a wide class of magnetically doped nitrides and oxides, such as (Ga,Mn)N [81], (Al,Cr)N [101], (Zn,V)O [70], (Zn,Co)O [68], and ( $\mathrm{Sn}, \mathrm{Co}) \mathrm{O}_{2}$ [59]. Another remarkable development is the observation of ferromagnetism persisting up to room temperature in ( $\mathrm{Zn}, \mathrm{Cr}) \mathrm{Te}$ [71].

\subsubsection{Manipulation of magnetic order via photonic or electric stimuli}

The enhancement of ferromagnetism (FM) and increase of the Curie temperature is a highly interesting topic in III-Mn-V DMS. The capability to turn on and off the mag- 
netic properties of these systems, especially on ultrafast timescales, would be tremendously useful for realizing future spintronic devices and multifunctional systems. The mean-field model of hole-mediated FM in III-Mn-Vs indicates that a change in the hole concentration induces a change in the FM interaction among Mn spins, leading to an increase or decrease of the transition temperature $\mathrm{T}_{C}$. The study of (photo) carrier-induced magnetic order is a very interesting and challenging subject. In II-VI-based DMS (CdMnTe), light-induced dynamic magnetic effects have been studied by the micro-SQUID technique, in which circularly polarized light has been utilized to yield spin-polarized carriers and thus magnetic polarons [3].

For the first time in III-Mn-V DMS, Koshihara and his coworkers have shown in 1997 that the strength of ferromagnetic spin exchange can be controlled by changing the hole concentration in $(\mathrm{In}, \mathrm{Mn}) \mathrm{As} /(\mathrm{Ga}, \mathrm{Al}) \mathrm{Sb}$ heterostructures by means of photogenerated carriers [47]. The consequence is an enhanced magnetization and, in some cases, a magnetic phase change. Using a $100 \mathrm{~W}$ halogen incandescent lamp, p-( $\left.\operatorname{In}_{0.94} \mathrm{Mn}_{0.06}\right) \mathrm{As} / \mathrm{GaSb}$ heterostructures were irradiated with white light of wavelengths ranging from $0.8 \mu(1.55 \mathrm{eV})$ to $1.4 \mu \mathrm{m}(0.88 \mathrm{eV})$. When samples were irradiated with light, an increase in magnetization occurred gradually (about 20 min to establish a steady state condition). The enhanced magnetization persisted at low temperatures $(\mathrm{T}<35 \mathrm{~K})$ even after the light was switched off. Light-induced changes in magnetization and Hall resistivity curves at $5 \mathrm{~K}$ are shown in figure 2.5. Before irradiation, the magnetization changes nonlinearly with an external magnetic field with no hysteretic behavior, consistent with proximity to the FM phase transition. After light irradiation, a hysteresis loop develops, indicative of a FM phase, induced 
by the presence of photo-generated excess holes.

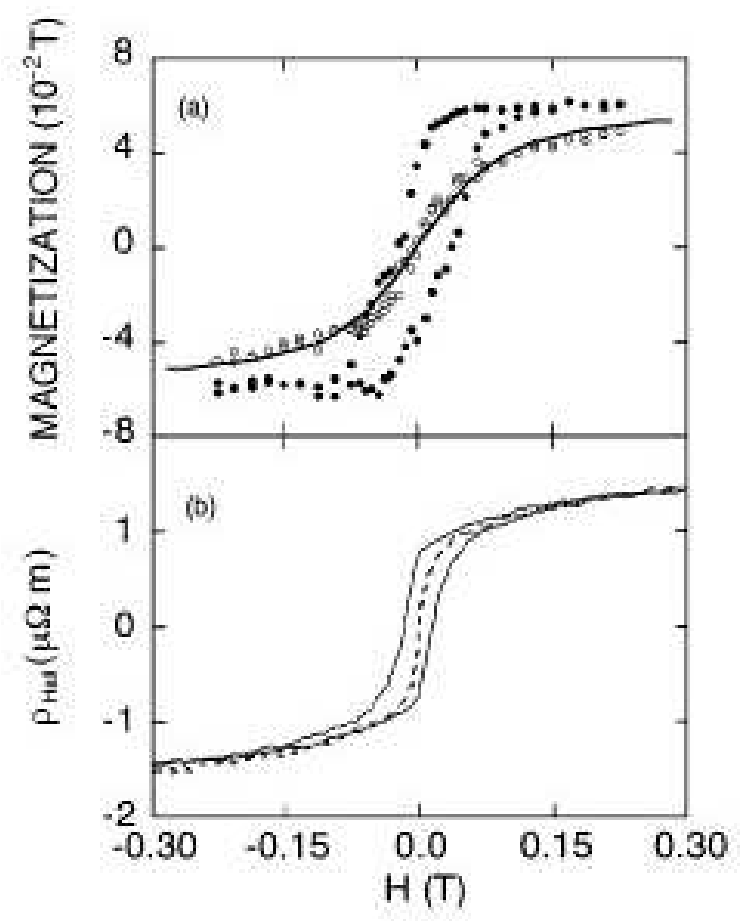

Figure 2.5: From ref. [47]: (a) Magnetization curves at $5 \mathrm{~K}$ observed before (open circles) and after (solid circles) light irradiation. Solid line shows a theoretical curve. (b) Hall resistivity $\rho_{\text {Hall }}$ ) observed at $5 \mathrm{~K}$ before (dashed line) and after (solid line) light irradiation.

Another way to modify the carrier concentration in semiconductors is by the field effect, offering the possibility to operate a magnetic field-effect transistor (FET). This has been demonstrated by Ohno and coworkers in 2000 on an insulating-gate FET structure with an (In,Mn)As channel [61]. Figure 2.6 shows a schematic of the FET structure, while figure 2.7 shows Hall resistivities (proportional to the magnetization perpendicular to the film) under three different gate biases, and the corresponding magnetization curves. The (In,Mn)As film is under tensile strain, making the easy axis (along which the magnetization aligns) perpendicular to the film plane, thus $\mathrm{R}_{\text {Hall }}$ measures magnetization hysteresis curves. At zero gate bias $\left(\mathrm{V}_{G}=0 \mathrm{~V}\right)$ the channel is slightly ferromagnetic at $22.5 \mathrm{~K}$ (soft hysteresis). 


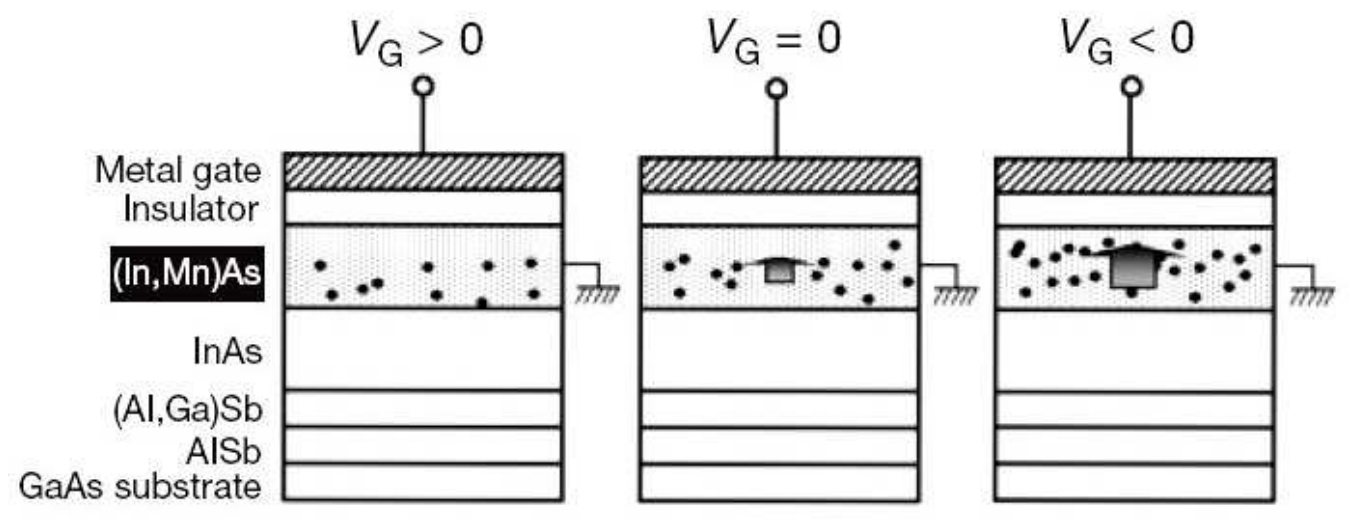

Figure 2.6: From ref. [61]: Field effect control of hole-induced FM in a magnetic semiconductor FET. Cross-section of a metal-insulator-semiconductor structure under gate bias $\mathrm{V}_{G}$. Negative $\mathrm{V}_{G}$ increases hole concentration, resulting in enhancement of the FM interaction among $\mathrm{Mn}$ ions, while positive $\mathrm{V}_{G}$ has opposite effect. The arrow shows schematically the magnitude of the Mn magnetization.
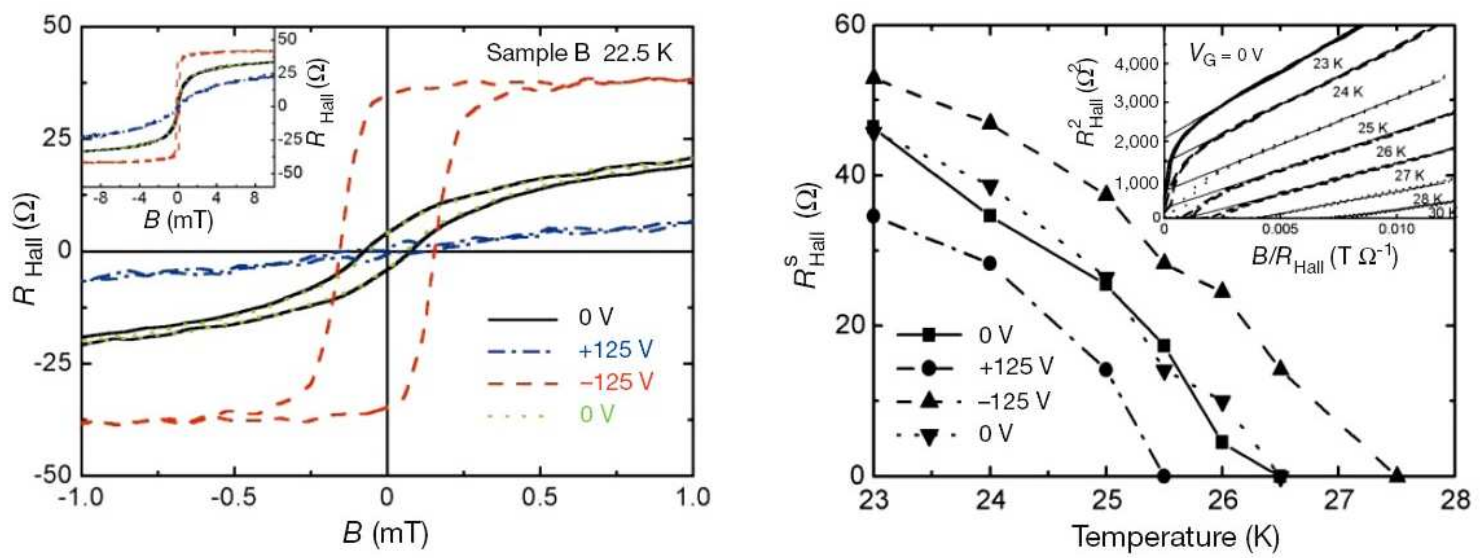

Figure 2.7: From ref. [61]: Left: $\mathrm{R}_{\text {Hall }}$ versus $\mathrm{B}$ field under three different gate biases at $22.5 \mathrm{~K}$ showing reversible, isothermal, electric field operation of magnetic FET. At $\mathrm{V}_{G}=$ $0 \mathrm{~V},+125 \mathrm{~V}$, and $-125 \mathrm{~V}$, the magnetic semiconductor layer is slightly FM, paramagnetic, and strongly FM, respectively. Right: Magnetization curves indicate $\pm 1 \mathrm{~K}$ modulation of $\mathrm{T}_{C}$ upon application of $\mathrm{V}_{G}= \pm 125 \mathrm{~V}$. 
Applying $\mathrm{V}_{G}=+125 \mathrm{~V}$ partially depletes the holes and reduces the FM interaction, resulting in paramagnetic behavior (no hysteresis). $\mathrm{V}_{G}=-125 \mathrm{~V}$ induces an accumulation of holes, and the sample shows clear hysteresis behavior.

Both of these two experiments rely on static measurements, in which the role of the Mn-hole correlations is unclear, as are the timescales of the microscopic, non-equilibrium processes taking place after light absorption. This strongly motivates the use of ultrafast experimental tools to push control of FM to technologically important, ultrafast timescales and to unravel the effects of the Mn-hole correlations in the photo-induced phenomena.

\subsubsection{Memory effects and magnetic memory read-out}

A practical aspect of interest for III-Mn-V DMS comes from the promise they hold over metallic magnetic devices for improved performance and integration into semiconductor spintronics. Both the semiconductor and the magnetic research communities pay close attention to the giant magneto-transport and magneto-optical effects in these systems, which bring about rich memory effects and allow for super-resolution magnetic state read-out.

A giant planar Hall effect was reported for the first time in 2003 by Tang and collaborators [87] in (Ga,Mn)As. This effect was accompanied by large switching effects in the Hall resistance that occurred at distinct magnetic fields. First, they noticed that a spontaneous transverse voltage develops because of the spin-orbit coupling in response to longitudinal current flow in epitaxial thin film (Ga,Mn)As devices. This effect occurs in the absence of an applied magnetic field, and is about 4 orders of magnitude stronger than in metallic ferromagnets [35]. Secondly, in a giant planar Hall resistance measurement, when 
sweeping a magnetic field oriented in-plane along a specific direction between $\pm 0.1 \mathrm{~T}$, large switching events occur at distinct values of the magnetic field (Fig. 2.8).

The anomalous switching behavior of the Hall resistance shown in figure 2.8 can be explained by a two jump sequence of the magnetization that normally lies along the [100] axis, first to a perpendicular, then to the negative [100] direction, as shown in figure 2.9. This switching occurs due to the competition between the external magnetic field and the internal magnetic anisotropy fields (which try to pin the magnetization along a preferred "easy axis" direction). The next two jumps complete the loop, and correspond to the magnetization continuing its rotation back to the initial position. This electrical transport experiment is remarkable in that it sheds light on the mechanism of magnetization behavior in $(\mathrm{Ga}, \mathrm{Mn}) \mathrm{As}$, and allows for extraction of the cubic and uniaxial anisotropy fields, while the high signal-to-noise ratio offers new possibilities for investigation of nanoscale spintronic devices.

In a magneto-optical experiment analogous to the magneto-transport measurements of Tang, a very similar effect has been reported by Kimel in 2005 [43]. They show that magnetic linear dichroism (MLD) in ( $\mathrm{Ga}, \mathrm{Mn}) \mathrm{As}$ is predominantly sensitive to the specific electronic states in the valence band that are responsible for the ferromagnetism, which they identify as close to the Fermi surface. MLD is the dichroism of linearly polarized light induced in a medium by an external magnetic field or magnetization. The effect originates from the difference of the dielectric function for light polarized parallel or perpendicular to the magnetization in the magnetic layer, and results in a rotation of the polarization of the reflected light. The MLD signal is proportional to $\mathbf{M}^{2}$, which itself has a term $\propto \bar{M}_{s p} \bar{H}$, 


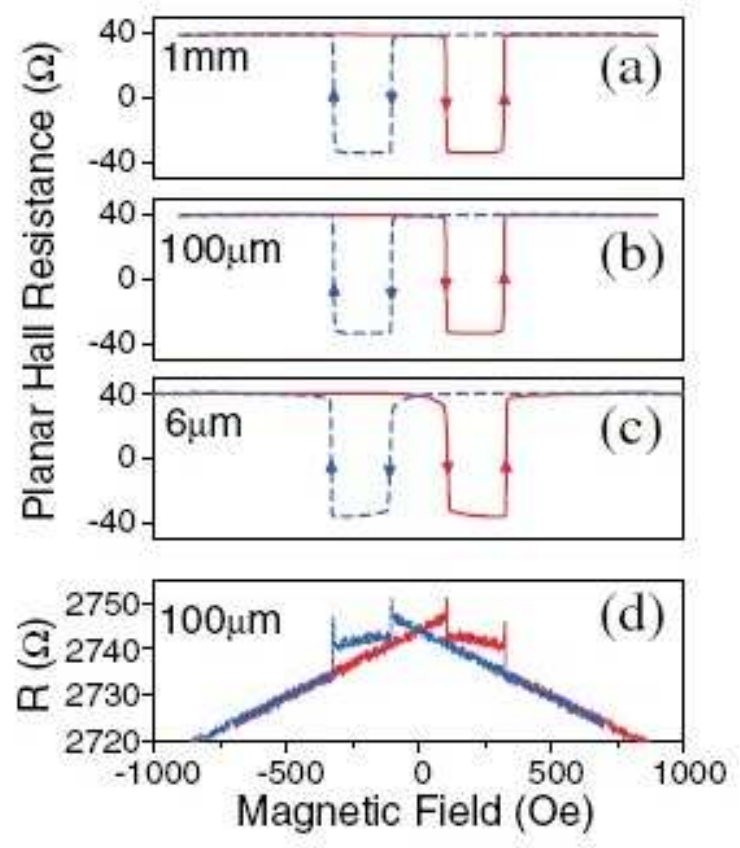

Figure 2.8: From ref. [87]: (a)-(c) Planar Hall resistance for Hall bars (1 mm, $100 \mu \mathrm{m}, 6$ $\mu \mathrm{m}$-wide) at $4.2 \mathrm{~K}$ as a function of in-plane magnetic field at fixed orientation. (d) Field dependent sheet resistance of a $100 \mu \mathrm{m}$-wide Hall bar.

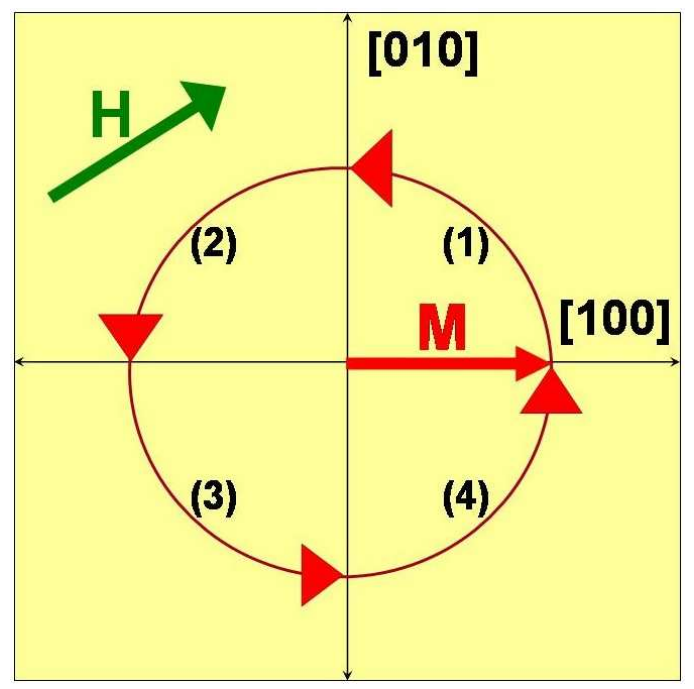

Figure 2.9: Large, switching events occur in the giant planar Hall resistivity of (Ga,Mn)As at external field sweep due to two $90^{\circ}$ jumps of the magnetization (that normally lies along an easy axis along [100]), first to a perpendicular, then to the negative direction. When the field is swept back, the next two jumps complete the loop, and correspond to the magnetization continuing its rotation back to the initial position. 
the scalar product between the spontaneous magnetization $\bar{M}_{s p}$ and the applied magnetic field $\bar{H}$.

The experiment by Kimel et. al. shows M-shaped MLD hysteresis loops as shown in figure 2.10. The signal exhibits jumps at $\mathrm{H}_{12}= \pm 9 \mathrm{mT}$ and $\mathrm{H}_{23}= \pm 110 \mathrm{mT}$, extremely sensitive to the orientation of the incoming light polarization. The shape of the hysteresis loops, similar to the one observed in the giant planar Hall resistance experiments, originates from the four-fold magnetic anisotropy of this material, with easy axes along both [100] and [010]. The process of magnetization reversal in an external magnetic field happens via $90^{\circ}$ jumps of the magnetization between the four easy axes directions that occurs when an external magnetic field is swept in the sample plane, as illustrated in figure 2.10 (a), causing abrupt changes in $\bar{M}_{s p} \bar{H}$ and the MLD signal. It is worth noting that the polar MOKE signal (Fig. 2.10(c)) shows a maximum around $1.55 \mathrm{eV}$ probe photon energy in (Ga,Mn)As. We will later use this effect and this photon energy in our ultrafast magneto-optical studies of $(\mathrm{Ga}, \mathrm{Mn}) \mathrm{As}$.

It has been shown that the magnetic anisotropy of $(\mathrm{Ga}, \mathrm{Mn}) \mathrm{As}$ films is largely controlled by epitaxial strains [77], with tensile and compressive strains inducing in-plane and out-of plane moment orientation, respectively. Bellow $\sim \mathrm{T}_{C} / 2[98]$ the magnetization can lie along one of four in-plane easy axis directions. Both of the experiments presented above allow for the unambiguous detection of the magnetization state, but are static equilibrium measurements with very slow read-out times. This naturally leads us to ask the question whether these states could be detected on an ultrafast, technologically relevant timescale, by a magneto-optical, ultrafast detection scheme. 

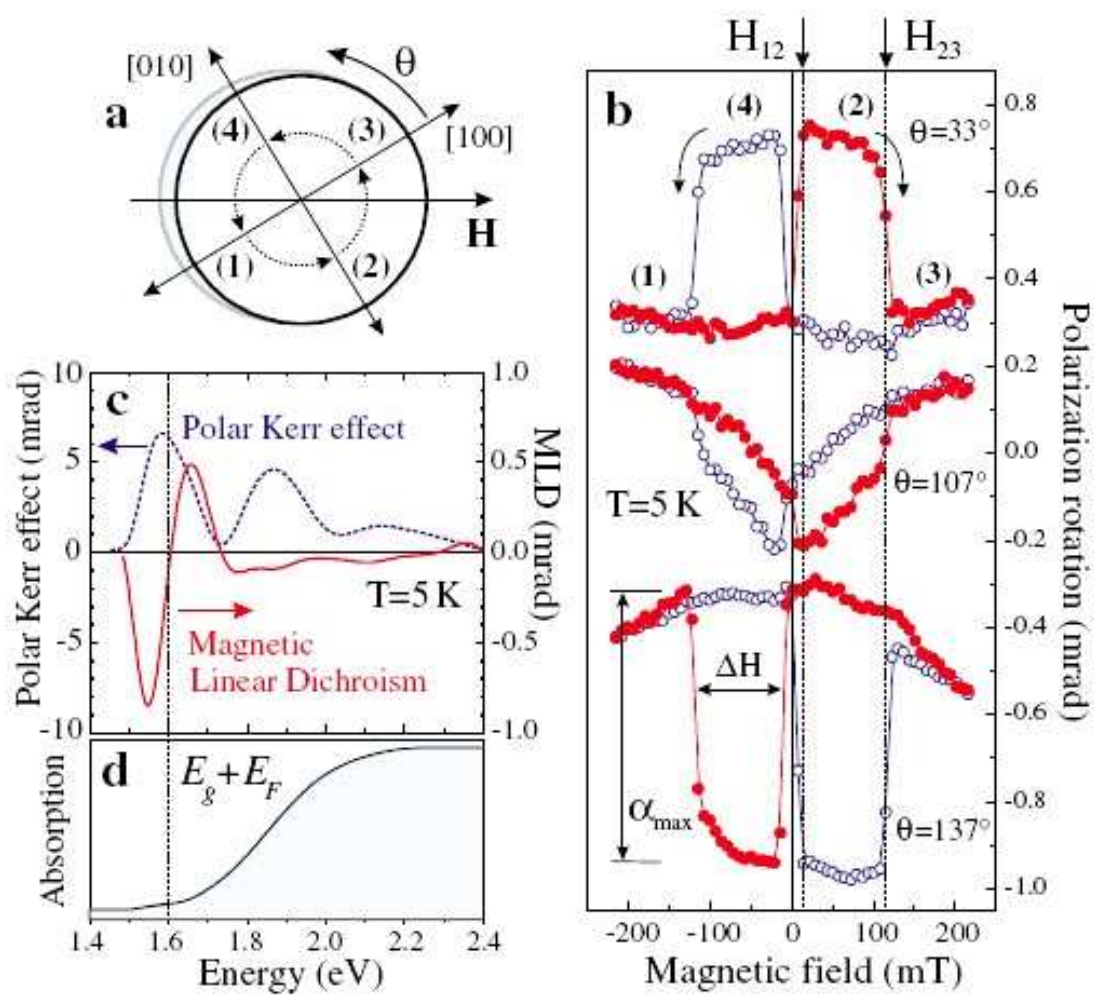

Figure 2.10: From ref. [43]: Magnetization switching in (Ga,Mn)As observed by magnetic linear dichroism. (a) (Ga,Mn)As sample orientation with respect to the applied magnetic field, and illustration of the four-step magnetization reversal process as consecutive $90^{\circ}$ jumps (shown by dotted arrows) between the four easy directions (1)-(4). (b) Field dependencies of the magnetic linear dichroism for different angles $\theta$ between the incident polarization and the [100] crystallographic direction, measured at a wavelength of $\lambda=815$ nm. (c) Spectra of polar magneto-optical Kerr effect and magnetic linear dichroism (at $\theta$ $\left.=135^{\circ}\right)$. (d) Absorption spectrum. 


\subsection{Magneto-optical Kerr effect}

We proceed to present here the basic concepts and techniques of magneto-optics spectroscopy which we use to study ultrafast dynamics of magnetization in magnetic materials. We first review the Kerr effect in ferromagnets, which is fundamental for our exploration of photoinduced modifications of magnetic order.

Faraday was the first to discover that when linearly polarized light travels through flint glass under an applied magnetic field, it undergoes a rotation of its plane of polarization proportional to the magnitude of the field [25]. This phenomenon, now called Faraday rotation, occurs both in non-magnets as well as in ferromagnets. In ferromagnets, the rotation is substantial $\left(10^{4}-10^{6} \mathrm{deg} / \mathrm{cm}\right)$ even for very small external fields, making them suitable for applications such as magnetic field sensors, optical isolators, and circulators [34]. A smaller polarization rotation is also observed in reflection, and is known as Kerr rotation.

When linearly polarized light reflects along the $\hat{z}$ direction perpendicular to the surface of a magnetized sample, with the magnetization vector along $\hat{z}$, it undergoes a rotation of the polarization plane. This comes as a consequence of the non-zero off-diagonal components of the dielectric susceptibility tensor:

$$
\tilde{\epsilon}=\left(\begin{array}{ccc}
\epsilon_{x x} & \epsilon_{x y} & 0 \\
\epsilon_{y x} & \epsilon_{y y} & 0 \\
0 & 0 & \epsilon_{x x}
\end{array}\right)
$$

where:

$$
\epsilon_{i j}=\epsilon_{i j}^{\prime}+i \epsilon_{i j}^{\prime \prime}=\epsilon_{i j}(M, \omega)
$$


These off-diagonal components translate into different microscopic responses to right and left circularly polarized light, the two normal modes of light propagation through the magnetized sample. $\sigma^{+}$and $\sigma^{-}$light see different propagation speeds and different absorption coefficients, and thus different reflection coefficients, $\mathrm{r}^{+}$and $\mathrm{r}^{-}$. This is the origin of the Kerr rotation and ellipticity.

Consider a plane wave of the form:

$$
\begin{aligned}
& \vec{E}=\vec{E}_{0} \exp [-i(\omega t-\vec{k} \vec{r})] \\
& \vec{H}=\vec{H}_{0} \exp [-i(\omega t-\vec{k} \vec{r})]
\end{aligned}
$$

governed by Maxwell's equations:

$$
\begin{aligned}
\nabla \times \vec{E} & =-\mu_{0} \frac{\partial \vec{H}}{\partial t} \\
\nabla \times \vec{H} & =\epsilon_{0} \epsilon \frac{\partial \vec{E}}{\partial t}
\end{aligned}
$$

Eliminating $\vec{H}$ we obtain:

$$
\vec{k}(\vec{k} \vec{E})-k^{2} \vec{E}+\epsilon_{0} \mu_{0} \omega^{2} \epsilon \vec{E}=0
$$

For light propagating along the $\hat{z}$ direction, also the direction of the magnetization M, we have $\vec{k} \vec{E}=0$ since $\vec{k}=(0,0, k)$ and $\vec{E}=\left(E_{x}, E_{y}, 0\right)$. We let $k_{0}=c \omega=\sqrt{\epsilon_{0} \mu_{0}} \omega$ and $N=\frac{k}{k_{0}}$ to obtain the eigenvalue equations:

$$
\begin{aligned}
& \left(N^{2}-\epsilon_{x x}\right) E_{x}-\epsilon_{x y} E_{y}=0 \\
& \epsilon_{x y} E_{x}+\left(N^{2}-\epsilon_{x x}\right) E_{y}=0
\end{aligned}
$$

and the eigenmodes of light propagation:

$$
\sigma^{ \pm}=E_{x} \pm i E_{x}
$$




$$
N_{ \pm}=\frac{k_{ \pm}}{k_{0}}=\sqrt{\epsilon_{x x} \pm i \epsilon_{x y}}
$$

Linearly polarized light incident along the $\hat{z}$ direction, can be decomposed into two in-phase circularly polarized light waves of equal amplitude $\sigma^{+}$and $\sigma^{-}$. At reflection off of the surface of a ferromagnet with magnetization $\mathrm{M}$ along $\hat{z}$, the two modes will experience different reflection coefficients:

$$
r_{ \pm}=\frac{N_{ \pm}-1}{N_{ \pm}+1}
$$

Thus if the incident light had $\vec{E}_{i n}=\left(E_{x}, 0,0\right)$, then the reflected light will be $\vec{E}_{r e f}=$ $\left(r_{x} E_{x}, r_{y} E_{x}, 0\right)$, effectively undergoing a rotation of complex angle

$$
\Theta_{K}=\theta_{K}+i \eta_{K}
$$

known as the Voigt vector. In this notation, $\eta_{K}$ represents the ellipticity, or the ratio of the minor to major axes of the reflected electric field ellipsoid, and $\theta_{K}$ represents the rotation of the major axis of the ellipsoid with respect to the incident polarization direction. Hence, when linearly polarized light is reflected from the sample surface, it becomes elliptically polarized and undergoes a rotation of the polarization vector. When the anisotropic indices are induced by magnetization, these effects are known as magnetic circular dichroism (MCD) and magneto-optical Kerr effect (MOKE). To be more precise, the particular geometry described above is referred to as polar geometry, and the effect is known as polar MOKE. If we carry out the calculations (for a detailed description, see [86]), in the case of negligible absorption, we obtain:

$$
\begin{aligned}
& \theta_{K}=-\frac{\epsilon_{x y}^{\prime}}{n\left(n^{2}-1\right)} \\
& \eta_{K}=-\frac{\epsilon_{x y}^{\prime \prime}}{n\left(n^{2}-1\right)}
\end{aligned}
$$




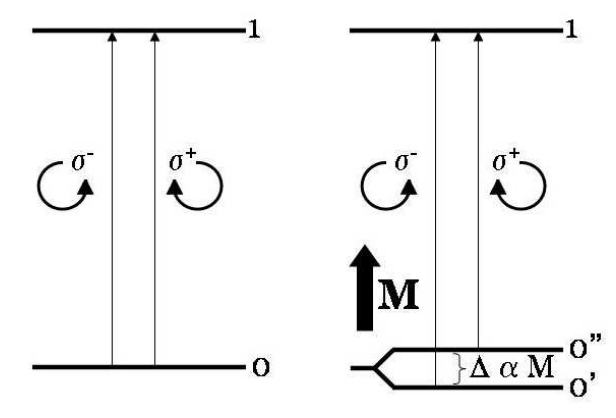

Figure 2.11: Energy levels split by an internal magnetic field.

To get an intuitive understanding on the dependence of $\epsilon_{x y}$ on the internal magnetization M, consider a transition between two levels 0 and 1 (Fig. 2.11). In the presence of the magnetic field generated by the internal magnetization $\mathrm{M}$, the 0 level is energy split into spin up and spin down levels. The energy difference is proportional to the magnetic field generated by the internal magnetization $\Delta \propto \mathbf{M}$. Thus the Kerr rotation originates from the selection rule differences between the allowed $\sigma^{+}$and $\sigma^{-}$transitions. Both real and imaginary parts of the off-diagonal tensor component $\epsilon_{x y}$ can be calculated to show that $\epsilon_{x y}^{\prime} \propto \Delta$ and $\epsilon_{x y}^{\prime \prime} \propto \Delta$, such that we can conclude:

$$
\begin{gathered}
\theta_{K}=-\frac{\epsilon_{x y}^{\prime}}{n\left(n^{2}-1\right)} \propto \Delta \propto \mathbf{M} \\
\eta_{K}=\frac{\epsilon_{x y}^{\prime \prime}}{n\left(n^{2}-1\right)} \propto \Delta \propto \mathbf{M}
\end{gathered}
$$

These formulas are well known in the literature, and indicate the fact that the Kerr rotation angle and ellipticity are proportional to the sample magnetization, and usually do not exceed 1 degree. In ultrafast applications, one measures MOKE and MCD signals by monitoring the time-resolved changes in the angle of rotation of the polarization vector and in ellipticity, 
which in effect reflect dynamics of the magnetization in the sample. As a true sign of their magneto-optical origin, the two signals should show the same magnetization dynamics, up to a proportionality constant (see Appendix A for such a verification on our signals).

We mention (for a derivation, see [103]) that the complex polar MOKE angle for an arbitrary incidence angle $\theta_{i}$ (and corresponding angle of refraction $\theta_{r}$ ) is given by:

$$
\begin{aligned}
& \Theta_{K}^{p}=\frac{\cos \theta_{i}}{\cos \left(\theta_{r}+\theta_{i}\right)} \times\left[i \epsilon_{x y} / \sqrt{\epsilon_{x x}}\left(\epsilon_{x x}-1\right)\right] \\
& \Theta_{K}^{s}=\frac{\cos \theta_{i}}{\cos \left(\theta_{r}-\theta_{i}\right)} \times\left[i \epsilon_{x y} / \sqrt{\epsilon_{x x}}\left(\epsilon_{x x}-1\right)\right]
\end{aligned}
$$

The situation is more complex in the UV, visible, and NIR parts of MOKE spectra for ferromagnetic (III,Mn)Vs, due to light absorption via interband transitions which are difficult to fully take into account. In addition, calculation of the MOKE angle requires a microscopic model of the susceptibility tensor.

Let's consider the main interactions that affect the magnitudes and features of MOKE signals, starting with the self consistent central field Hamiltonian $\mathrm{H}_{0}$, and adding on the correlation energy $\mathrm{H}_{e e}$, spin orbit interaction $\mathrm{H}_{S L}$, crystal field interaction $\mathrm{H}_{C F}$, and finally the exchange interaction $\mathrm{H}_{e x}$ and Zeeman splitting $\mathrm{H}_{z}$ (Fig. 2.12). The nondiagonal elements of the dielectric tensor are sensitive to these level splittings. These interactions remove the degeneracy of the multiplet state in solids, leading to different transitions for the $\sigma^{+}$and $\sigma^{-}$photons. Therefore, the MOKE and MCD signals, sensitive to the different transitions of the polarized photons, depend on the transition strength, exchange coupling, and spin orbit coupling strength. This, in turn, makes them sensitive to, for example, $d$ states in transition-metal ions because of the strong coupling of these states. Thus, the MOKE NIR spectrum in GaMnAs can be tied to spin-polarized electronic transitions 


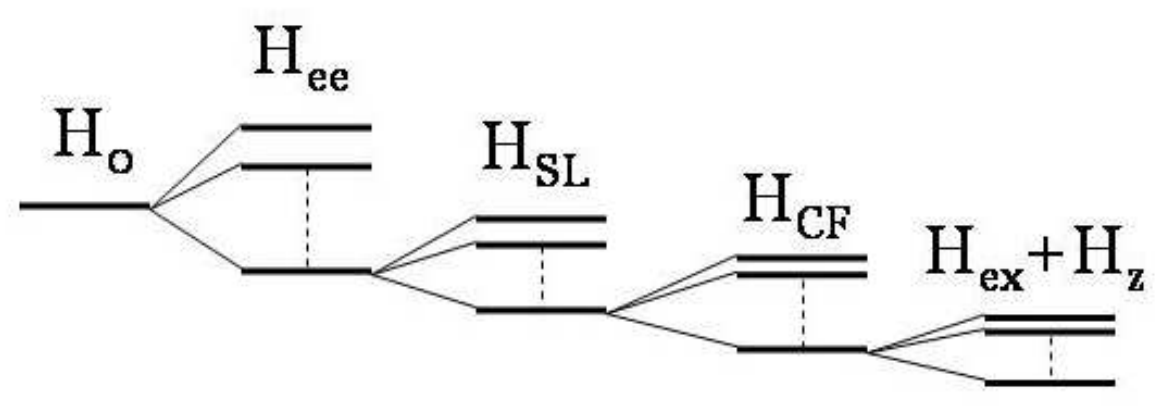

Figure 2.12: Energy levels split ordered in microscopic interactions decreasing strength.

related to $\mathrm{Mn} 3 d$ local moments, and effectively used to probe the system magnetization dynamics.

So far we have limited our discussion exclusively to polar MOKE configuration effects (i.e., when the incident light is along the $\hat{z}$ direction, perpendicular to the surface of the sample, and the magnetization vector lies along the same $\hat{z}$ direction). It must be mentioned that there are three different types of MOKE: polar, longitudinal and transverse, depending on the direction of the magnetization vector $\mathbf{M}$ with respect to the surface of the material and the plane of incidence of the input optical beam, as depicted in Figure 2.13.

In polar MOKE, the Kerr effect is simple and occurs for incident light polarized in the p-plane ( $\vec{E}$ parallel to the plane of incidence) or s-plane ( $\vec{E}$ perpendicular to the plane of incidence). Radiation incident in either of these linearly polarized states is, on reflection, converted into elliptically polarized light, and the major axis of the ellipse is rotated slightly with respect to the principal plane. Similar effects also occur in transmission, although their observation usually requires a thin film, since most magnetic materials are opaque in their magneto-optically active spectral regions. The sign and magnitude of these effects are 

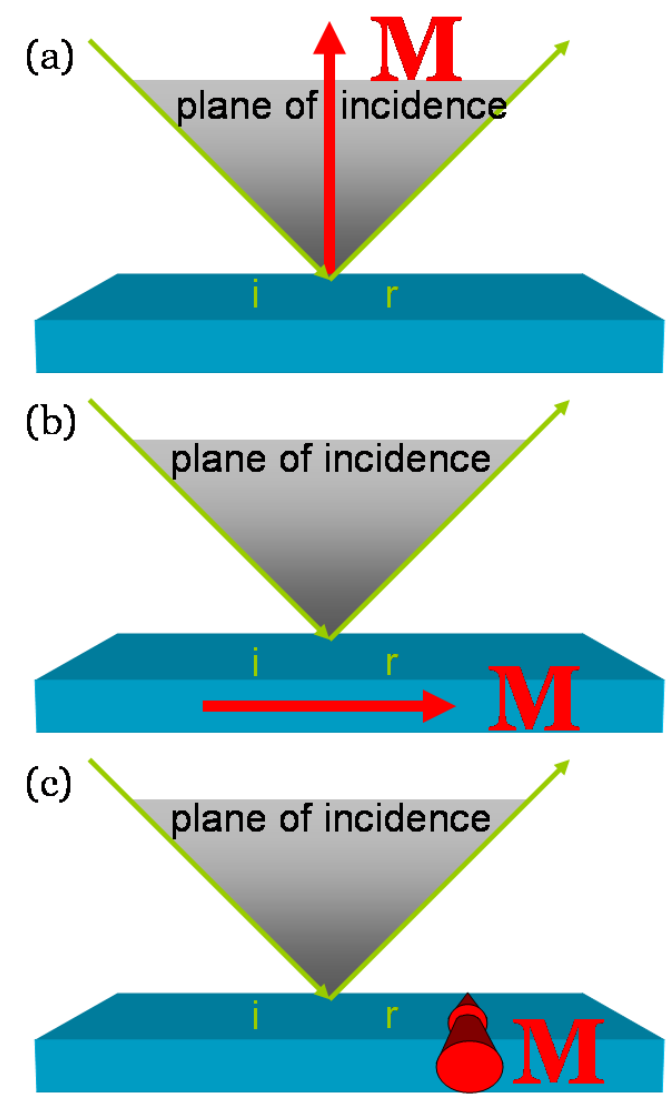

Figure 2.13: Three MOKE configurations: (a) polar, (b) longitudinal, and (c) transverse. 
proportional to $\mathrm{M}$ and its direction. In the longitudinal case, the magnetization vector is oriented along the sample surface, parallel to the plane of incidence. The effect is otherwise analogous to the effect in the polar MOKE configuration.

The transverse case is quite different from the previous two. First, there is only an effect for radiation polarized in the p-plane $(\vec{E}$ in the plane of incidence). Second, the reflected radiation remains linearly polarized and there is only a change in reflected (or transmitted) amplitude, such that as $\mathbf{M}$ changes sign from $+\mathbf{M}$ to $-\mathbf{M}$ the reflectivity changes from $\mathrm{R}+\Delta \mathrm{R}$ to $\mathrm{R}-\Delta \mathrm{R}$. No transverse MOKE effect is observed at normal incidence.

\subsection{Femtosecond magneto-optics}

In the following section, we give a short review of ultrafast magneto-optics and major results obtained over the past years. So far we have discussed static MOKE/MCD effects, but let's turn our attention to dynamic effects, due to pump-induced variations of the magnetization, and MOKE/MCD signals. It turns out that the interpretation of transient magneto-optical signals on the femtosecond (fs) timescale and under weak pump perturbations is not trivial. In a pump-probe experiment, the pump creates a transient carrier population, which can change the oscillator strength of the relevant optically-coupled states seen by the probe, thus changing the prefactors in the MOKE and MCD dependence on magnetization. As shown in the previous section, the complex MOKE vector is directly proportional to the magnetization $\mathrm{M}$ :

$$
\Theta_{K}=\theta_{K}+i \eta_{K}=\propto \epsilon_{x y}^{\prime}+i \propto \epsilon_{x y}^{\prime \prime}=f_{1}(\omega) M+f_{2}(\omega) M=\tilde{F} M(t)
$$


Due to the transient carrier population, the complex proportionality factor $\tilde{F}$ can also become time-dependent, such that the proportionality between MOKE and MCD signals is lost. In other words, if in the static case we have:

$$
\frac{\Delta \Theta}{\Theta_{0}}=\frac{\Delta \theta}{\theta_{0}}=\frac{\Delta \eta}{\eta_{0}}=\frac{\Delta M}{M_{0}}
$$

then in the dynamic case we have:

$$
\frac{\Delta \Theta}{\Theta_{0}}=\frac{\Delta \tilde{F}}{F_{0}}+\frac{\Delta M}{M_{0}} \neq \frac{\Delta M}{M_{0}}
$$

and the induced magneto-optical signals no longer reflect only magnetization dynamics. The equality and the time-independent proportionality of MOKE and MCD signals is only valid while $\Delta \tilde{F} / F_{0} \ll \Delta M / M_{0}$.

There have been experiments in which the time--resolved MOKE signal was shown not to be of magnetic origin alone, but rather from dichroic bleaching effects in the first 500 fs after pump excitation [45]. In this case, only after the initial 0.5 ps did the temporal profiles of MOKE and MCD data begin to overlap (Fig. 2.14). It was concluded that state filling effects induced by the pump prevailed during the first $0.5 \mathrm{ps}$ of the transient magneto-optical response, masking any change in magnetization. This is supported by a second experiment on Ni films [67], in which it was argued that such bleaching effects originate from the degenerate pump-probe techniques.

In a different set of experiments on ferromagnetic metals, in particular time-- and spectrally-resolved measurements on $\mathrm{CoPt}_{3}[29,9]$ the authors differentiate between the contributions of the spin and the charge populations in the magneto-optical signals (Fig. 2.15). This is achieved by carefully separating the dynamics of the diagonal and the nondiagonal elements of the time-dependent dielectric tensor. They conclude that after the 


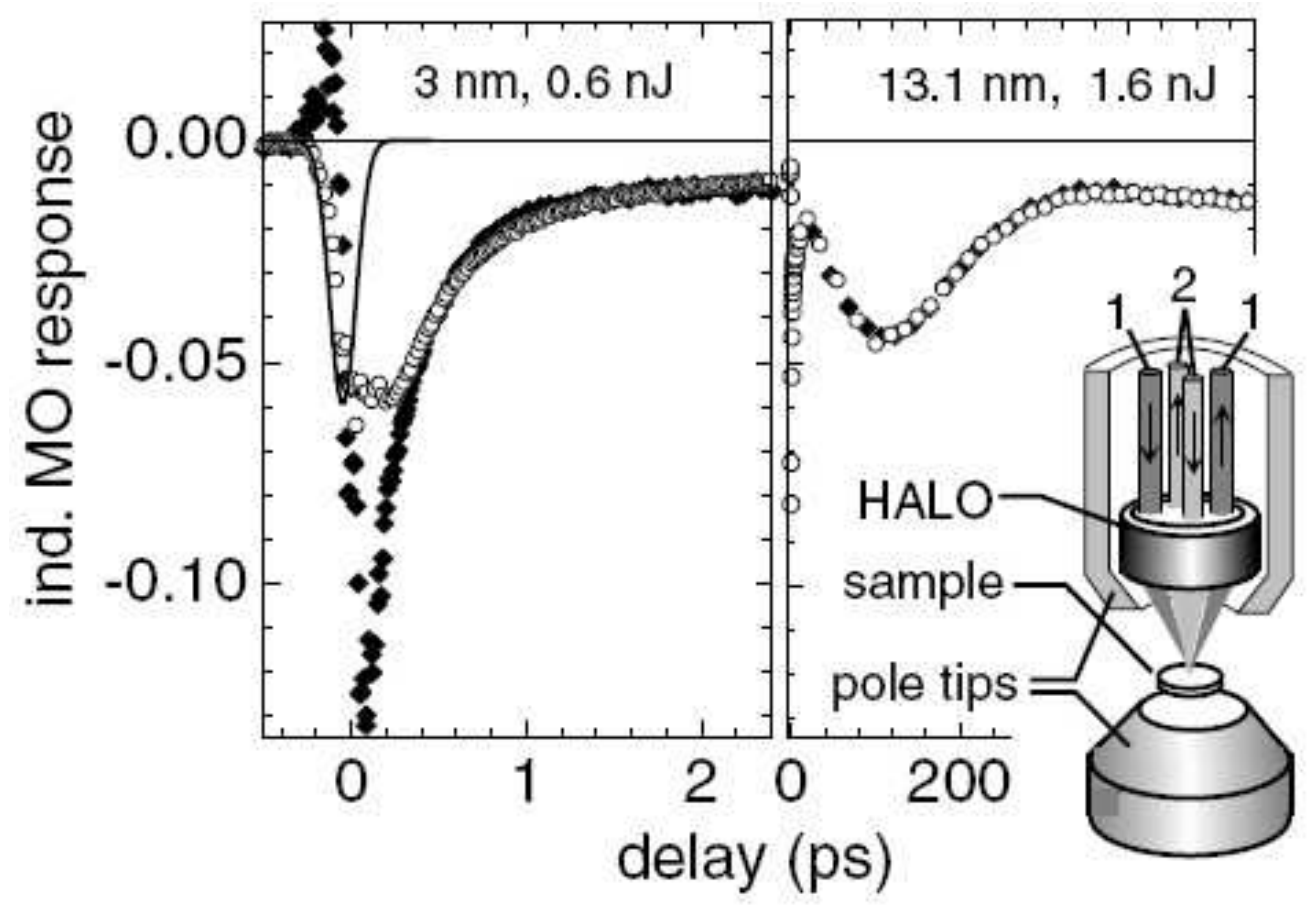

Figure 2.14: From ref. [45]: Comparison of the induced ellipticity (open circles) and rotation (filled diamonds) as a function of pump-probe delay time in Ni films. The thick line represents the pump-probe cross correlation trace. The inset depicts the experimental configuration with pump (1) and probe (2) beams. 
thermalization time of the electrons $(50 \mathrm{fs})$, the dynamics of the real and the imaginary parts of the Voigt vector are identical. In addition, their relative variation is 10 times larger than that of the diagonal elements of the tensor, which allows one to infer that the spins dominate the magneto-optical response. The lack of dichroic bleaching in $\mathrm{CoPt}_{3}$ indicates that the transient magneto-optical signals depend on the particular material studied.

One can conclude from these experiments that MOKE and MCD are very powerful tools to study ultrafast magnetization dynamics. However, for unambiguous conclusions, especially related to femtosecond spin phenomena, a non-degenerate pump-probe setup is desirable in order to avoid artifacts from transient optical effects such as dichroic bleaching. Moreover, one needs to be careful that the relative variations of the dielectric tensor offdiagonal elements are much greater than those of the diagonal elements (i.e., MOKE/MCD relative variations are much larger than differential reflectivity variations). 

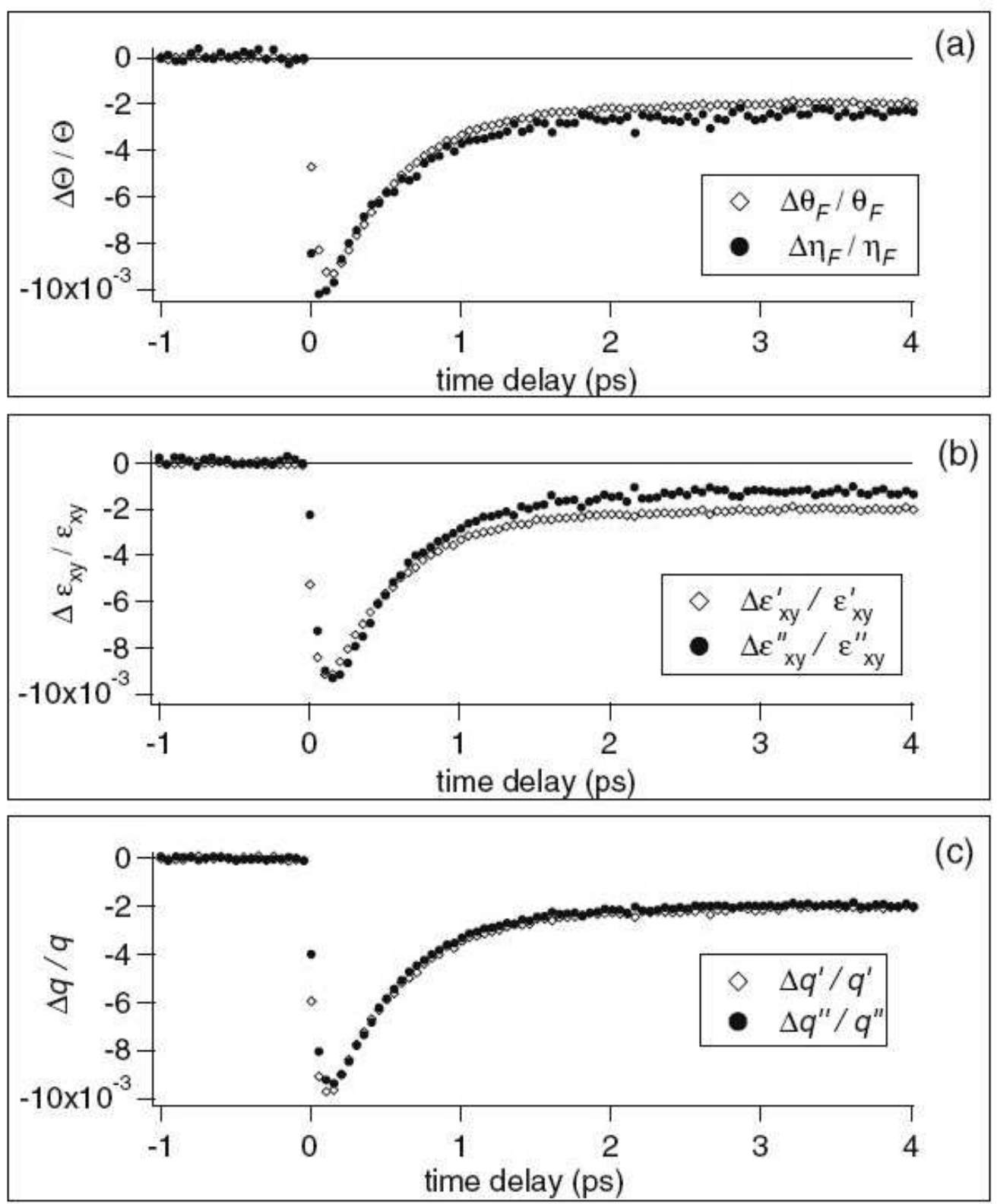

Figure 2.15: From ref. [29]: (a) Time-resolved Faraday magneto-optical signals $\Delta \theta / \theta$ and $\Delta \eta / \eta$ for long temporal delays. (b) Temporal evolution of the real part $\Delta \epsilon_{x y}^{\prime} / \epsilon_{x y}^{\prime}$ and the imaginary part $\Delta \epsilon_{x y}^{\prime \prime} / \epsilon_{x y}^{\prime \prime}$ of the nondiagonal element of the dielectric tensor. (c) Temporal evolution of $\Delta q^{\prime} / q^{\prime}$ and $\Delta q^{\prime \prime} / q^{\prime \prime}$ also retrieved from data in (a). The fact that $\Delta q^{\prime} / q^{\prime}$ and $\Delta q^{\prime \prime} / q^{\prime \prime}$ follow exactly the same dynamics for $\mathrm{t} \geq 150$ fs shows that the residual difference between real and imaginary parts observed in (b) can be attributed to the (small) nonmagnetic contributions to the dynamics of nondiagonal tensor elements. 


\subsection{Conclusions}

In this chapter, we reviewed properties of dilute magnetic semiconductors with a particular focus on III-Mn-V materials as promising candidates for spintronics applications and mutifunctional devices. We learned that the ferromagnetism in these systems is carrier-mediated, and discussed key experiments on ( $\mathrm{Ga}, \mathrm{Mn})$ As that motivated us in our

work. Even if (Ga,Mn)As is still far from becoming a mainstream spintronics material due to its low $\mathrm{T}_{C}$, basic research on this material helps develop applications for room temperature ferromagnetic semiconductors. In the second part of this chapter, we presented the concepts we used for probing dynamics in magnetic materials at ultrafast timescales, such as magneto-optical Kerr effect and femtosecond magneto-optics. 


\section{Chapter 3}

\section{Experimental Setup}

\subsection{Outline}

In this chapter, we present a practical description of our ultrafast magnetization dynamics experiments. First, we discuss the semiconductor samples under study and the characterization of their basic properties. At the heart of our experiments are a $\sim 100 \mathrm{fs}$ laser system and a $2 \mathrm{~K}, 7 \mathrm{~T}$ magneto-optical cryostat which are detailed next. This is followed with a presentation of our experimental layout, including a description of the alignment process. We then give an overview of our experiments and show how ultraviolet (UV) pump, near-infrared (NIR) probe MOKE spectroscopy directly reveals magnetization dynamics in the samples. Finally, a description of data collection is presented along with relevant calculations used for the interpretation of the experimental data. 


\subsection{Samples}

In order to study control of magnetism in ferromagnetic semiconductors by exciting transient carriers, we investigate several different samples. All the samples are of the $\mathrm{Ga}_{1-x} \mathrm{Mn}_{x} \mathrm{As}$ family, but are grown by different techniques, in different film thicknesses, and some have co-dopants. The equilibrium solubility of Mn in GaAs is known to be at most $10^{19} \mathrm{~cm}^{-3}$. If higher Mn concentrations are employed under equilibrium growth conditions, MnAs clusters begin to form within the GaAs host material. However, non-equilibrium growth techniques, such as low temperature molecular beam epitaxy (LT-MBE), or ionimplantation followed by pulsed laser melting (II-PLM) allow one to obtain alloys with Mn concentrations of up to nearly $9 \%$ [104]. $\mathrm{Ga}_{1-x} \mathrm{Mn}_{x}$ As becomes ferromagnetic for $x>1 \%$.

Two of our samples (called A and B in the following) were grown by LT-MBE by Professor Jacek K. Furdyna at the University of Notre Dame. Of these two samples, sample A was thin film $\mathrm{Ga}_{1-x} \mathrm{Mn}_{x} \mathrm{As}$, and sample $\mathrm{B}$ was even thinner film, quasi-2D nanolayer of $\mathrm{Ga}_{1-x} \mathrm{Mn}_{x}$ As. Three other samples (C, D, and E) were provided to us by Professor Oscar Dubon at the University of California at Berkeley. These thin film samples, grown by II-PLM, were $\mathrm{Ga}_{1-x} \mathrm{Mn}_{x} \mathrm{As}, \mathrm{P}$ co-doped $\mathrm{Ga}_{1-x} \mathrm{Mn}_{x} \mathrm{As}_{1-y} \mathrm{P}_{y}$ and $\mathrm{S}$ co-doped $\mathrm{Ga}_{1-x} \mathrm{Mn}_{x} \mathrm{As}_{1-y} \mathrm{~S}_{y}$. Figures 3.1 and 3.2 show typical structures for the LT-MBE and IIPLM samples, and Mn concentration profiles, while table 3.1 details the overall samples characteristics.

In the following, we describe details of the growth and characterization as performed by the Furdyna and Dubon groups. In the case of the two LT-MBE grown samples, A and B, first a GaAs buffer layer was grown on a semi-insulating [001] GaAs substrate un- 

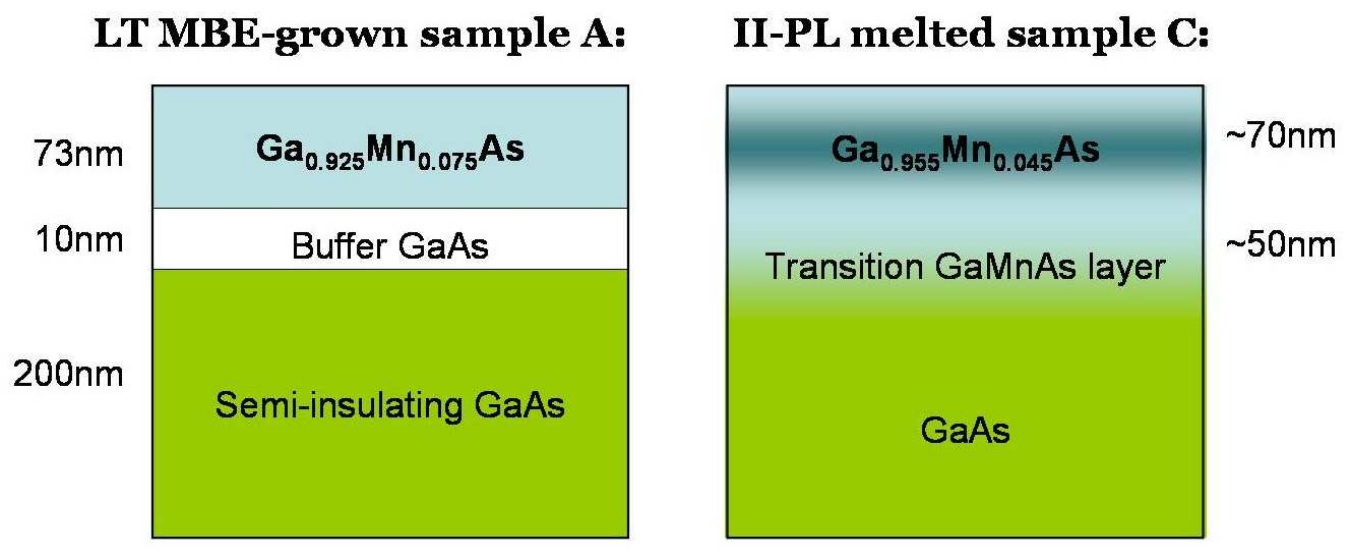

Figure 3.1: Structures of samples A and C

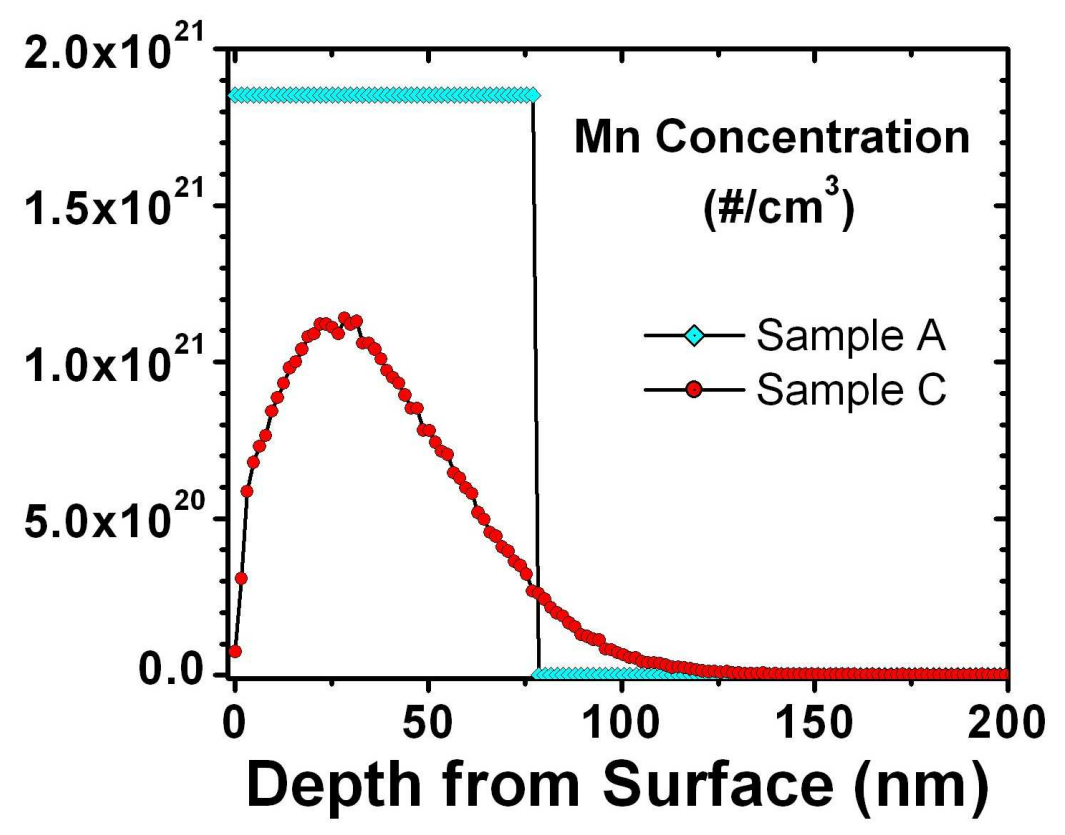

Figure 3.2: Comparison of Mn concentrations versus depth from the sample surface in LT-MBE sample A, and II-PLM sample C. 
Table 3.1: Properties of $\mathrm{Ga}_{1-x} \mathrm{Mn}_{x}$ As samples investigated in this work

\begin{tabular}{|c|c|c|c|c|c|c|c|}
\hline Sample & $\begin{array}{c}\text { Grown } \\
\text { by: }\end{array}$ & $\begin{array}{c}\mathrm{Mn} \\
\%\end{array}$ & $\begin{array}{c}\mathrm{p} \\
\left(10^{20} \mathrm{~cm}^{-3}\right)\end{array}$ & $\begin{array}{c}\mathrm{T}_{C} \\
(\mathrm{~K})\end{array}$ & $\begin{array}{c}\text { anisotropy } \\
\text { direction }\end{array}$ & $\begin{array}{c}\text { film thick- } \\
\text { ness }(\mathrm{nm})\end{array}$ & $\begin{array}{c}\text { co- } \\
\text { dopant }\end{array}$ \\
\hline $\mathrm{A}$ & LT-MBE & 7.5 & 3 & 77 & {$[100] /[1 \overline{10}]$} & 73 & none \\
$\mathrm{B}$ & LT-MBE & $\sim 9$ & 3 & 140 & {$[100] /[110]$} & 10 & none \\
$\mathrm{C}$ & $\mathrm{II}-\mathrm{PLM}$ & 4.5 & 4.4 & 90 & {$[110]$} & $\approx 100$ & none \\
$\mathrm{D}$ & II-PLM & 4.1 & $1-6$ & 80 & {$[001]$} & $\approx 100$ & $\mathrm{P} 2.2 \%$ \\
$\mathrm{E}$ & II-PLM & 3.6 & $<1$ & 70 & {$[100] /[110]$} & $\approx 100$ & $\mathrm{~S} 0.3 \%$ \\
\hline
\end{tabular}

der standard growth conditions $\left(590^{\circ} \mathrm{C}\right)$. After that the substrate temperature was reduced to $200-300^{\circ} \mathrm{C}$ and a $10 \mathrm{~nm}$ and $2 \mathrm{~nm}$, respectively, LT GaAs buffer layer was deposited. The $\mathrm{Ga}_{1-x} \mathrm{Mn}_{x}$ As layer was then grown on top of the buffer layer. For epitaxial films grown on [001] GaAs, the strain via the magnetoelastic interaction enforces the easy axis of magnetization to lie in the thin film layer plane. Reflection high-energy electron diffraction (RHEED) was used to monitor the surface reconstruction during growth, which was always carried out under As-stabilized conditions. Since the lattice constant of zinc-blende GaMnAs increases with the Mn concentration $\mathrm{x}$, the Mn concentration $\mathrm{x}$ was determined using $\mathrm{x}$-ray diffraction $(\mathrm{XRD})$ as discussed in $[64,76]$. The experimental determination of the hole concentration by room-temperature Hall effect measurements yielded $\mathrm{p} \approx 3 \cdot 10^{20} \mathrm{~cm}^{-3}$ for samples A and B. While Hall measurements on GaMnAs are affected by complications arising from the anomalous Hall effect (AHE), in the case of room temperature measurements on samples with low Mn and carrier concentrations these complications have been shown to be small and thus provide a useful estimate of the hole concentration. Sample A characterization measurements to determine doping profile, in-plane temperature dependence of magnetization along the four easy axes, and hysteresis loops along those respective directions at $5 \mathrm{~K}$ are presented in figures 3.2 through 3.4 . 


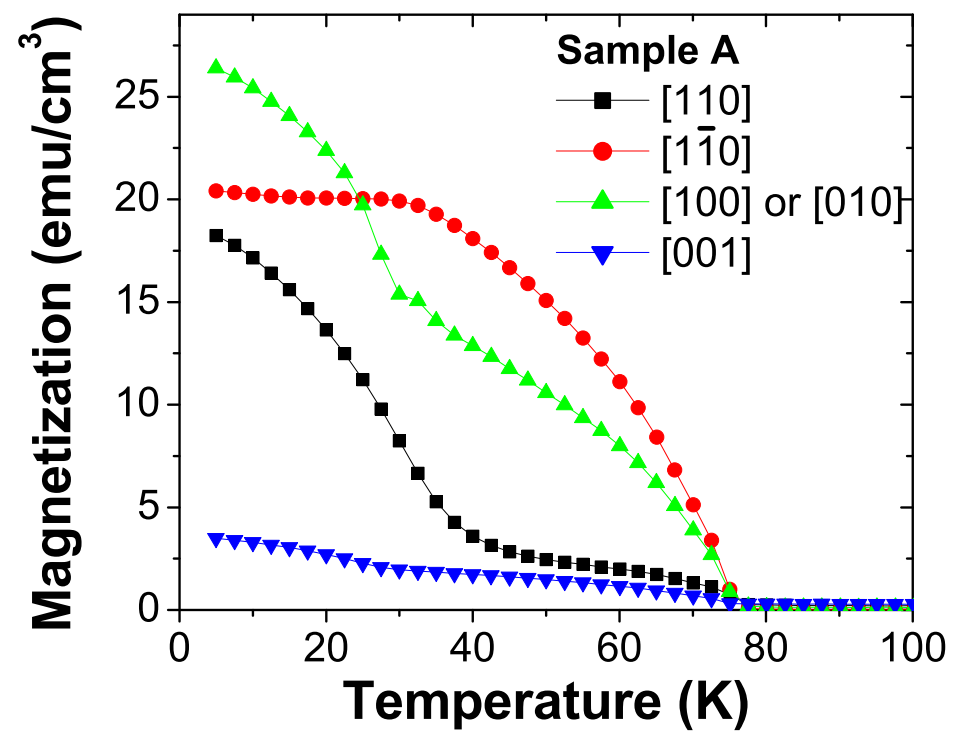

Figure 3.3: Temperature dependence of magnetization along the four in plane easy axes.

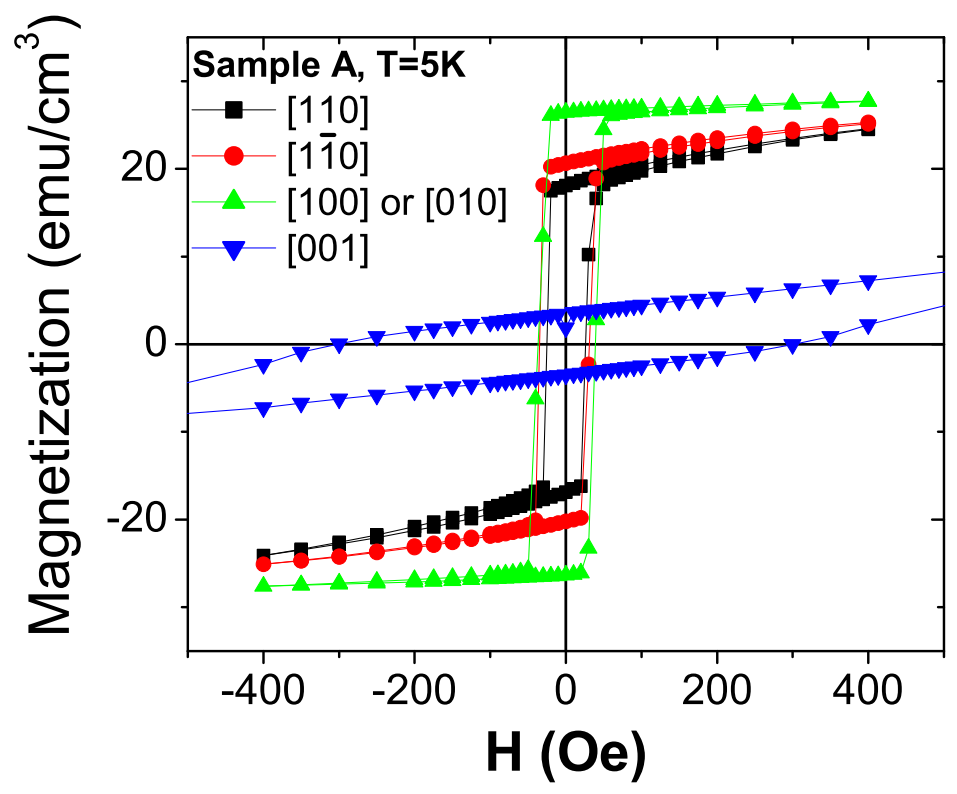

Figure 3.4: Hysteresis loops at $5 \mathrm{~K}$ with $\vec{H}$ along the four in-plane easy axes. 
The other three samples, C-E, were synthesized using the combination of ion implantation and pulsed-laser melting. In effect, $\mathrm{Ga}_{1-x} \mathrm{Mn}_{x} \mathrm{As}$ was synthesized by $50 \mathrm{keV}$, $1.5 \times 10^{16} / \mathrm{cm}^{2} \mathrm{Mn}^{+}$implantation into semi-insulating GaAs followed by irradiation with a single pulse from a $\operatorname{KrF}(\lambda \approx 248 \mathrm{~nm})$ excimer laser at a fluence of $0.3 \mathrm{~J} / \mathrm{cm}^{2}$. RBS/PIXE ion channeling analysis demonstrated that the fraction of Mn residing on substitutional sites (MnGa) was typically 75-85\% depending on the Mn implanted dose and laser fluence. During pulsed laser melting, the ion-implanted region of the film melts, solidifies epitaxially, and then cools to room temperature within a few hundred nanoseconds. As a result of this high-temperature processing $\left(\mathrm{T}_{\text {Melt }}=1511 \mathrm{~K}\right.$ for GaAs), films produced using II-PLM are free from interstitial $\mathrm{Mn}_{I}$. In fact, the remainder of the Mn atoms is incommensurate with the lattice. The $\mathrm{P}-$ and $\mathrm{S}-$-co-doped samples were synthesized by performing further implantation of $\mathrm{P}$ or $\mathrm{S}$ ions prior to pulsed-laser melting. Etching in concentrated $\mathrm{HCl}$ for 5-20 minutes was used to remove excess Mn from the surface that was present in Ga droplets and in surface oxides. It was verified that this etching did not affect the ferromagnetic or electrical transport properties of the films. Sample C characterization measurements of doping profile, in-plane temperature dependence of magnetization along the two easy axes, hysteresis loop at $5 \mathrm{~K}$, and sheet resistivity versus temperature are presented in figures 3.2 , and $3.5-3.7$.

Mn, $\mathrm{P}$ and $\mathrm{S}$ concentrations and substitutional fractions were determined by the combination of secondary ion mass spectrometry (SIMS) and ion beam analysis. Magnetization measurements were performed using a SQUID magnetometer in an applied field of 50 Oe while de transport was measured in the van der Pauw geometry. 


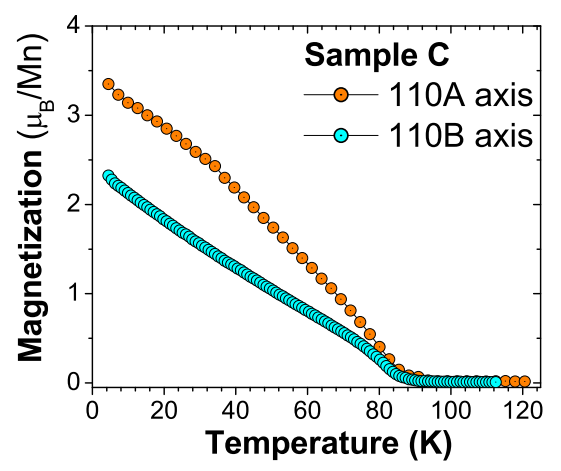

Figure 3.5: Temperature dependence of magnetization along the two in plane easy axes.

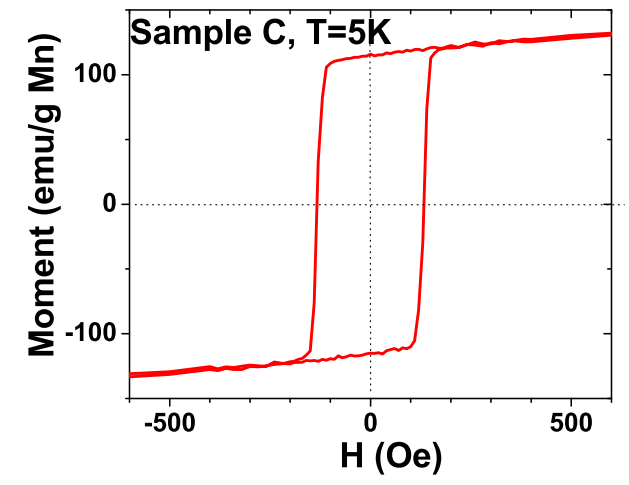

Figure 3.6: Hysteresis loop at $5 \mathrm{~K}$ with $\vec{H}$ along 110A.

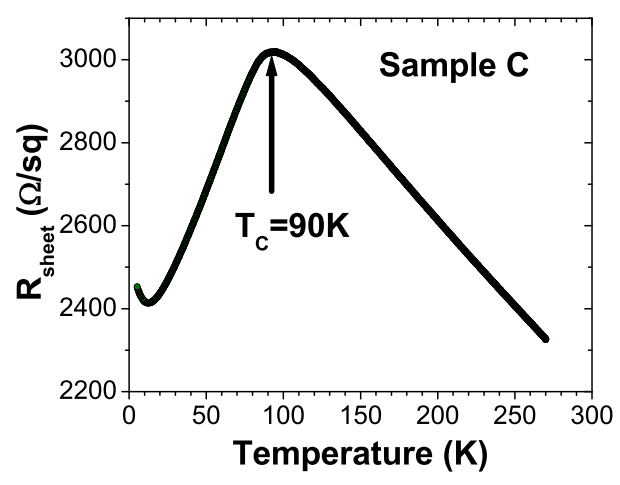

Figure 3.7: Temperature dependence of sheet resistivity showing a dip at $T \sim 15-20 K$. 


\subsection{The ultrafast laser system}

Transient, coherent optical experiments in ferromagnetic semiconductors with femtosecond time resolution necessitate a source of ultrashort light pulses. High repetition rates and a long-term stability of this source are also important for optimal signal-to-noise ratio. The laser system used in our experiments is a Coherent Mira 900. It uses a Ti-doped sapphire crystal, pumped by $8 \mathrm{~W}$ of continuous wave (CW) visible light from a Coherent Innova 300 Argon-ion laser. It produces 154 fs pulses, with the wavelength tunable from $720 \mathrm{~nm}$ to $810 \mathrm{~nm}$.

The Mira 900 is mode-locked by "Kerr lens modelocking", a technique that utilizes changes in the spatial profile of the beam produced by self focusing from the optical Kerr effect in the Ti:sapphire crystal. Self focusing is a non-linear optical effect where the refractive index $\mathrm{n}$ depends linearly on the light intensity I, $n=n_{0}+n_{2} I$. Accordingly, a Gaussian beam profile experiences a variation of the optical path length when passing through such a medium similar to that given by a lens. By adding an aperture into the

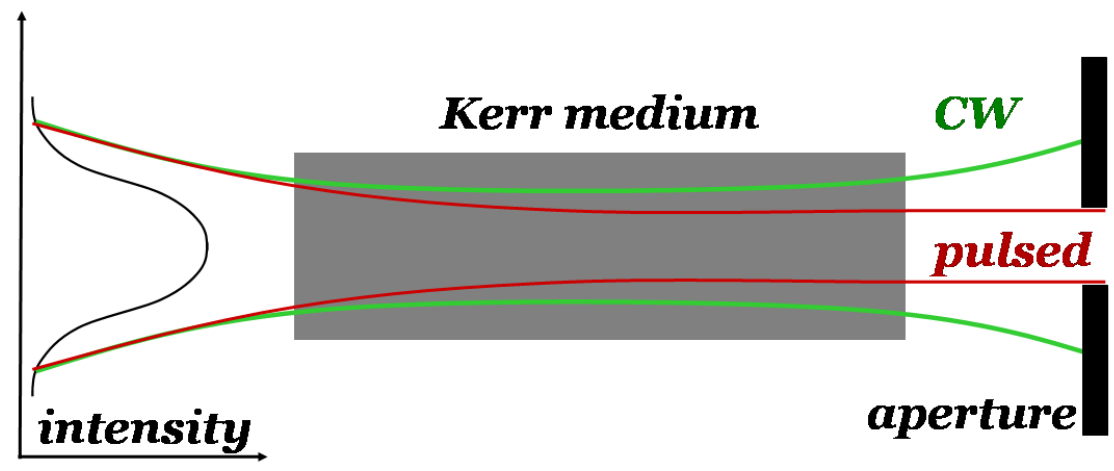

Figure 3.8: Schematics of Kerr mode-locking 
cavity that preferentially selects the self-focused mode (see schematics in figure 3.8), self focusing results in higher round trip gain for the modelocked (high peak power) versus the CW (low peak power) operation. The pulse repetition rate as determined by the laser cavity length is $80 \mathrm{MHz}$ (12.5 ns between pulses). The pulse train is continuously monitored for stability using a fast photodiode connected to a $400 \mathrm{MHz}$ Tektronix $2465 \mathrm{~B}$ oscilloscope.

The NIR laser pulses used in our experiments are obtained after minute alignment of the laser cavity (Fig. 3.9). The laser pulse spectrum is characterized using an Acton Research Corporation Spectra Pro 750 spectrometer. Typical pulse spectra were centered around a wavelength of $785 \mathrm{~nm}$, with a bandwidth of $7 \mathrm{~nm}$ (Fig. 3.10). At this wavelength, the Mira laser outputs a power of up to $600 \mathrm{~mW}$.

To measure the duration of the laser pulses, we employed intensity autocorrelation measurements, which lets the pulse measure itself. In this measurement, the pulse is split into two components, which are variably delayed with respect to each other, and spatially overlapped in a second-harmonic-generation (SHG) non-linear optical crystal. One obtains signal light at twice the frequency of the input light. The field envelope of the autocorrelation pulse is given by:

$$
E^{S H G}(t, \tau) \propto E(t) E(t-\tau)
$$

This field has an intensity proportional to the product of the intensities of the two input pulses, delayed by $\tau$. By varying the delay $\tau$ between the two pulses, we obtain the intensity envelope measured by the detector, which allows us to extract the pulse duration:

$$
A^{S H G}(\tau) \propto \int_{-\infty}^{\infty} I(t) I(t-\tau) d t
$$

In our setup, we utilized a $0.5 \mathrm{~mm}$ thick beta-barium borate $\beta \mathrm{BaB}_{2} \mathrm{O}_{4}$ (BBO) crystal, 

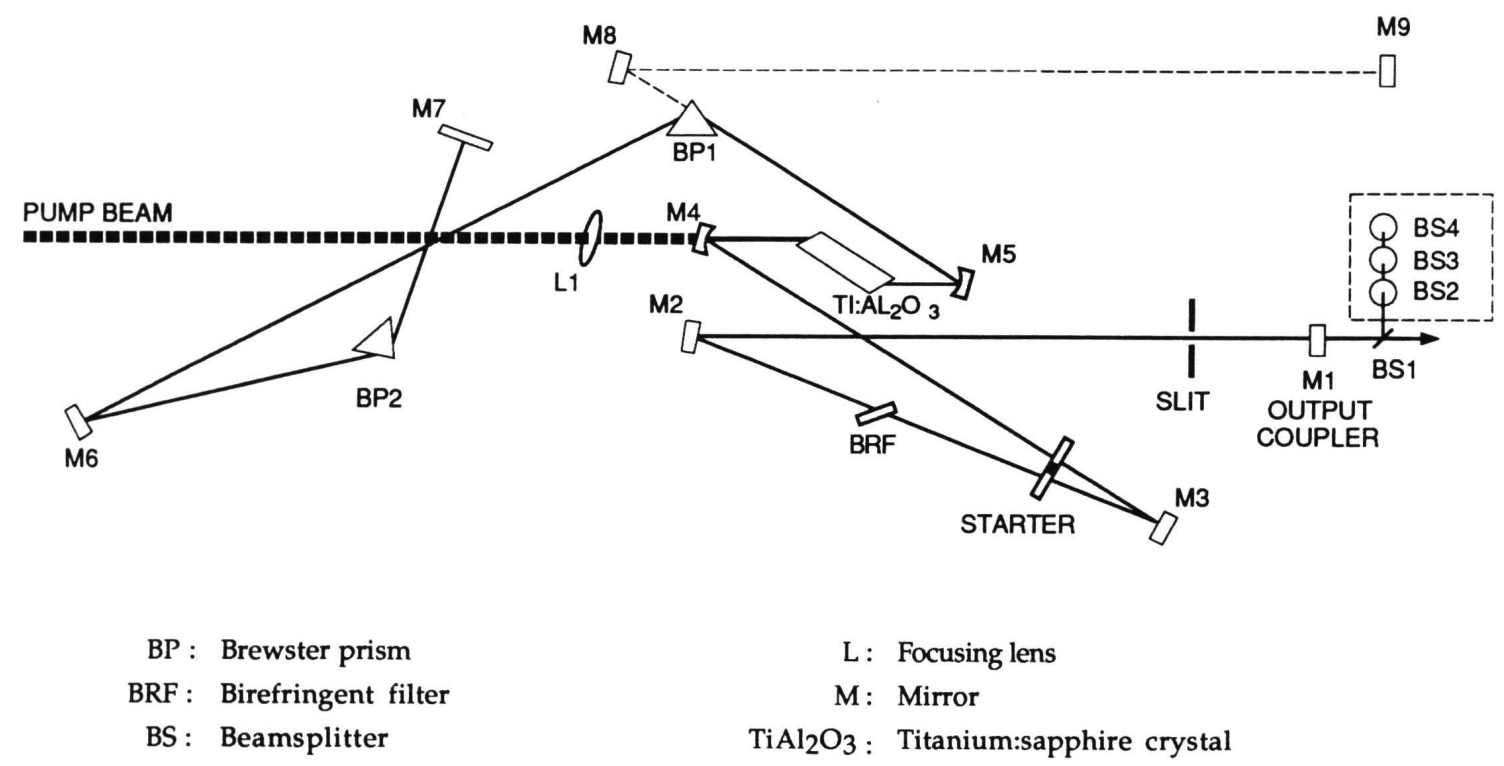

Figure 3.9: Optical schematics of Mira 900 laser cavity from the "Operator's Manual: The Coherent Mira Model 900 Laser" by Coherent, Inc.

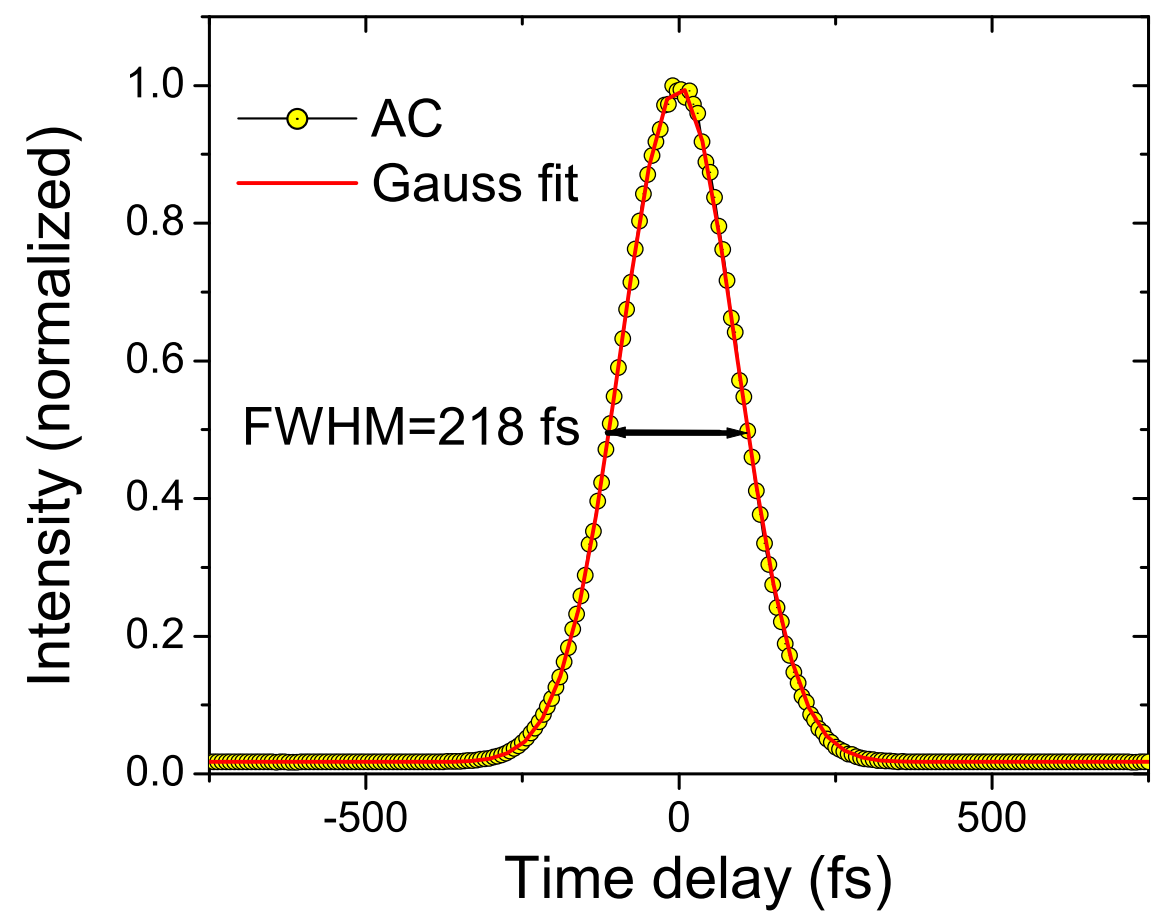

Figure 3.10: Typical laser autocorrelation shows FWHM $=218 \mathrm{fs}$. Since this is the convolution of identical laser pulses, the typical NIR pulse duration $\Delta \tau=\sigma / \sqrt{2}=154$ fs. 
designed for second harmonic generation of $800 \mathrm{~nm}$ light. The two beams were incident on the crystal at slightly different angles, so that the SHG beam at $400 \mathrm{~nm}$ propagated in a background-free direction in between the incident beam directions. The autocorrelation signal was detected with a Hammamatsu 931A photo-multiplier tube, and sent to a Stanford Research Systems SR830 lock-in amplifier. One of the two beams was chopped using an HMS Light Beam Chopper 221, whose frequency was then referenced by the lock-in amplifier. The result of a typical autocorrelation is shown in figure 3.10, along with a Gaussian fit. From this, we determined that the pulse has a typical temporal width $\Delta \tau$ of $154 \mathrm{fs}$. The spectral width is $7 \mathrm{~nm}$ at $785 \mathrm{~nm}$, corresponding to a frequency width $\Delta \nu=0.00341 \mathrm{fs}^{-1}$, such that the bandwidth product is $\Delta \nu \Delta \tau=0.5251$. This is within $18 \%$ percent of the transform limit $(0.4413)$. These temporal pulse characteristics were consistently reproduced throughout our experiments as long as the wavelength was kept fixed. 


\subsection{Magneto-optical cryostat}

At the heart of our experiments, we used a split-coil magneto-optical cryostat built by Janis Research Company, Inc. This piece of equipment is essential in ensuring low temperature control, high magnetic field, and optical access to our samples. The temperature control is enabled by use of a variable temperature insert built into the helium (He) reservoir of the dewar (Fig. 3.11). The lower portion of this insert, containing the sample, fits inside the bore of the split coil superconducting magnet. A split coil design is required for the magnet - as opposed to a simple solenoid design - in order to provide an axis for optical access to the sample, separate from the axis required for the sample holder.

All of our experiments were done using this magnet, which operates between 0 and $7 \mathrm{~T}$, and exhibits a field uniformity of better than $1 \%$ within a $1 \mathrm{~cm}$ diameter volume. The optical axis used was along the axis of the superconducting coils, with the magnetic field perpendicular to the sample plane. A second, separate optical axis was also available along the gap between the magnet coils, for experiments in the Voigt geometry with the magnetic field along the sample surface.

The superconducting coils of the magnet are enclosed in a stainless steel can, filled with liquid He at $4 \mathrm{~K}$ to ensure the magnet is superconducting. This He can is surrounded by a liquid Nitrogen (LN2) jacket at $77 \mathrm{~K}$, of which it is separated by a vacuum enclosure $\left(10^{-6}\right.$ Torr $)$. The LN2 jacket is separated from ambient conditions by a continuation of the vacuum chamber. The variable temperature insert (VTI) containing the sample runs along the axis of the helium can, but is separated from it by vacuum (Fig. 3.11). At the same time the VTI is connected to the He reservoir through a capillary tube which can be 


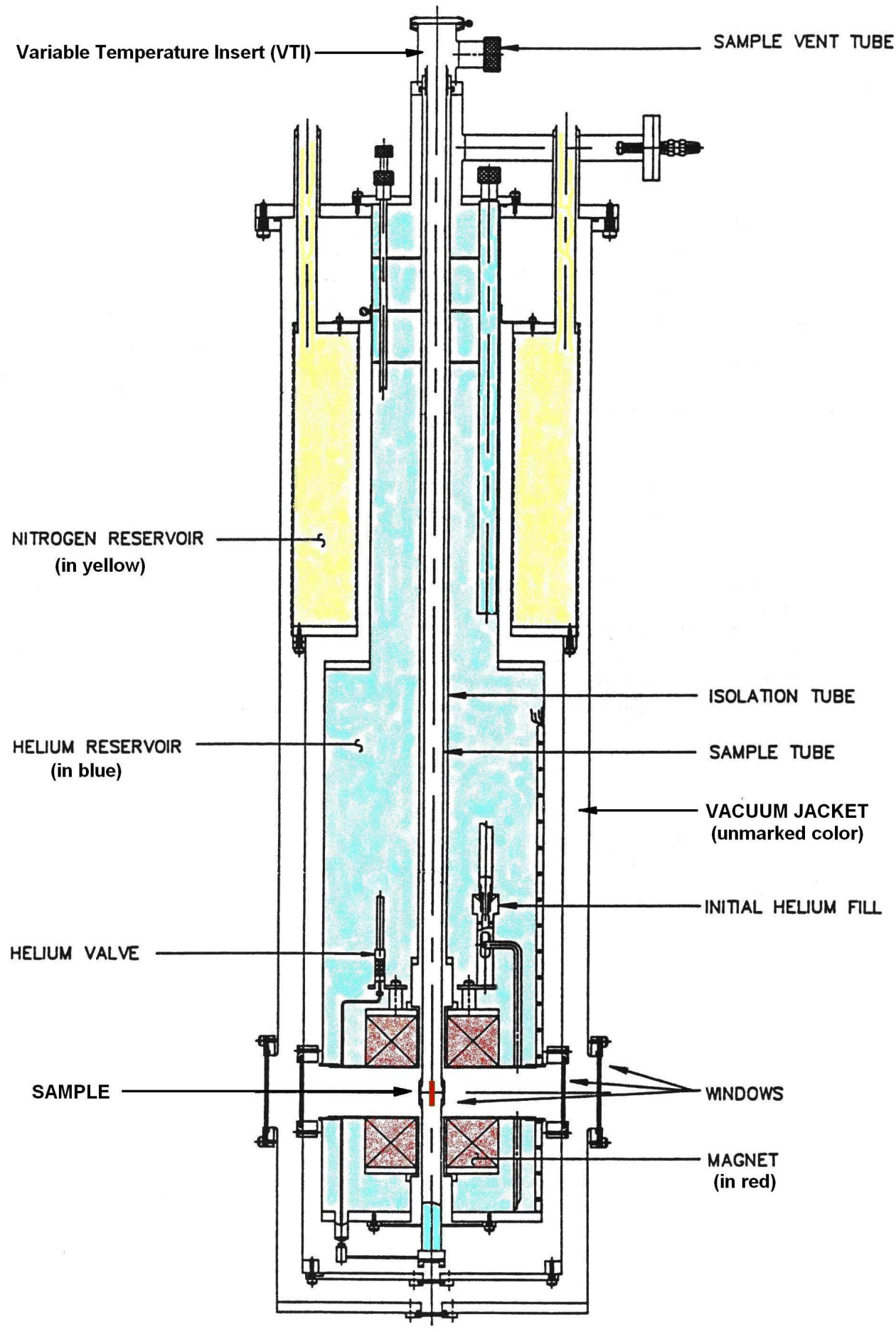

Figure 3.11: Magnet cryostat for magneto-optical experiments, including variable temperature insert (enhanced Janis cryostat manual drawing). 
opened and closed with an externally operated needle valve. This valve allows the flow of He from the reservoir to the VTI. The temperature of the sample cell inside the VTI can be controlled from as low as $1.5 \mathrm{~K}$ up to $300 \mathrm{~K}$ by a combination of He flow regulation through the needle valve, vacuum pumping on the sample cell, and heating of the sample stick. Once the He flow has been set, a temperature sensor on the sample stick can be used to automatically control the sample temperature via a feedback loop between the heater and the sample, and maintain a steady set temperature. The magnet itself is manually controlled via an external power source from 0 to $\pm 7 \mathrm{~T}$ with $0.01 \mathrm{~T}$ precision. Together with the He level monitors, these controllers are shown in figure 3.12.

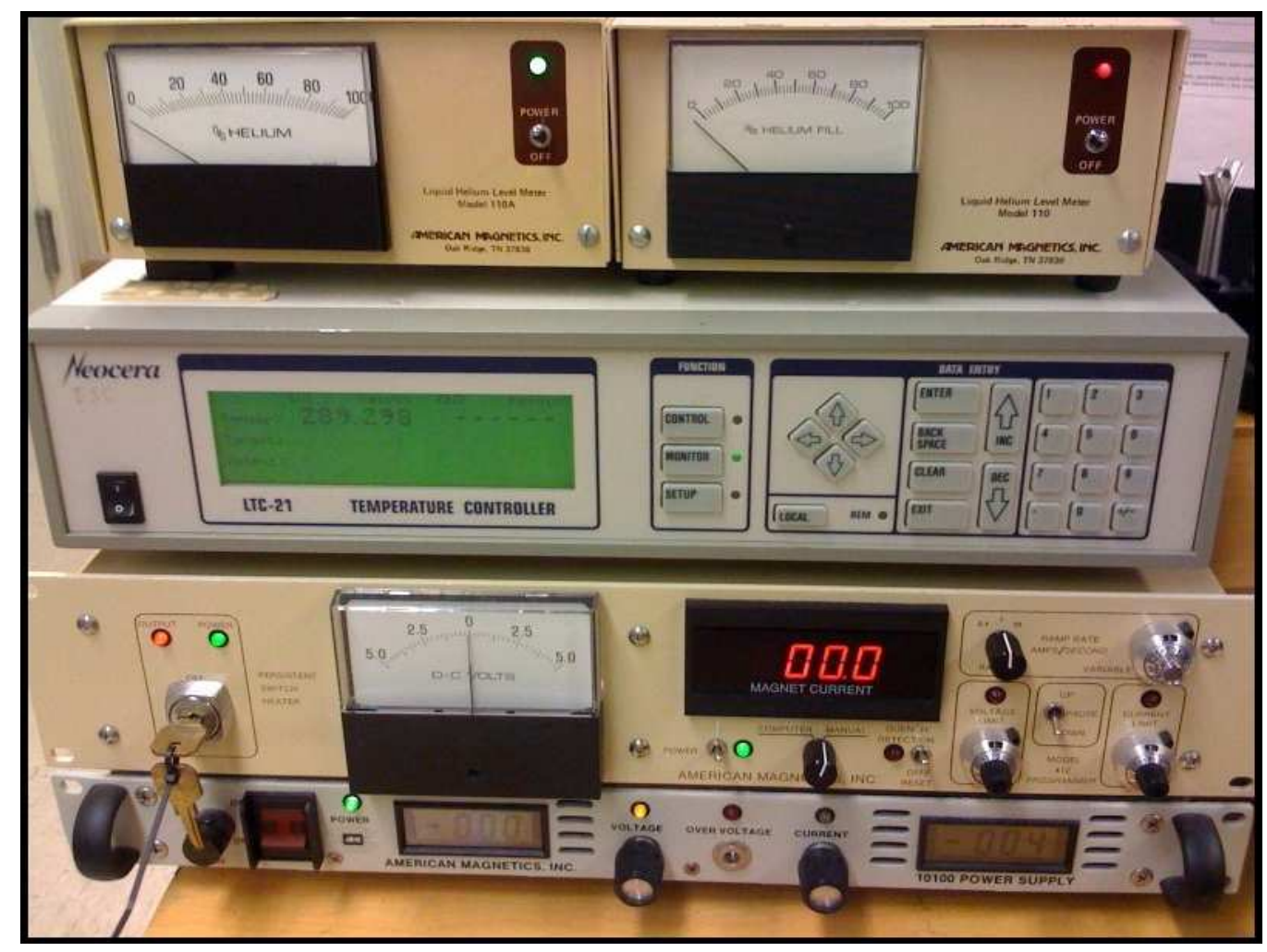

Figure 3.12: He level sensors at the top indicate when He needs to be refilled. Next down, the temperature controller allows for temperature readout and control via a feedback loopserved variable power heater. At the bottom, the magnet power source allows for $\pm 0.01 \mathrm{~T}$ control of the magnetic field from $0 \mathrm{~T}$ to $\pm 7 \mathrm{~T}$. 


\subsubsection{Cooling the magnet}

The cool down process begins with pumping on the outer vacuum chamber for 24 hours or more. This vacuum chamber is connected to all vacuum spaces inside the cryostat, such that they all get pumped simultaneously (Fig. 4.11). Next, in order to prepare the liquid He reservoir for precooling with LN2, we purge this space thoroughly with clean, dry nitrogen gas. Thus we ensure that no water, liquid or vapor, is left to freeze in the system or in the needle valve. In the next major step, we pre-cool the He reservoir with LN2 (as a cheaper alternative to directly cooling with He). While pumping on the sample cell side, the needle valve is opened, which should prompt the mechanical pump to "gurgle", indicating that LN2 is entering the sample cell. The needle valve is then closed and the pump quiets down. This operation is repeated a few times. After a 6-12 hours delay - in which time the magnet has reached thermal equilibrium - the LN2 is pumped out of the He can into the LN2 jacket by pressurizing the He can with He gas. The LN2 jacket is topped off with LN2, while the He can is left to warm up slightly for boil off of any remaining LN2 (another 6-12 hours). After thoroughly purging the He can with He gas, the magnet enclosure can finally be cooled down to $4 \mathrm{~K}$ by filling it up with liquid He. At the same time, the sample cell is cooled down by pumping on it while flowing He through the needle valve. The VTI and the superconducting magnet are now ready for operation. Both the He can and the LN2 jacket need to be refilled every $\approx 40$ hours. 


\subsection{Experimental layout}

In this section we detail the experimental setup used for UV pump, NIR probe MOKE spectroscopy, shown schematically in Figure 3.13. A list of essential equipment can be found at the end of this section in table 3.2. The Mira 900 laser output $(600 \mathrm{~mW}$, $\lambda=800 \mathrm{~nm}, 154 \mathrm{fs}$ ) is prepared as s-polarized, and then divided into two beams by a 90:10 beam splitter. The $10 \%$ beam eventually becomes the NIR probe beam, while the $90 \%$ beam eventually becomes the UV pump beam.

The $10 \%$ beam, used as the probe beam, passes through a half wave plate (HWP) mounted on a Sigma Koki rotation stage controlled by a computer, which allows us to control the angle of its polarization. It is then sent onto a retro-reflector (RR) mounted through a magnetic base onto a translational stage, also computer-controlled. This stage allows to induce the necessary time delay between the pump and the probe pulses to study the magnetization dynamics induced by the pump. The delay stage (Newport ILS 150 MVTP, controlled by a Newport Universal Motion Controller/ Driver ESP 300) has a $150 \mathrm{~mm}$ travel range corresponding to up to 1 ns pulse delay in double pass mode, and a $0.5 \mu \mathrm{m}$ positioning accuracy corresponding to 3.3 fs resolution. In practice, given our 154 fs pulse duration, the smallest step we typically use is $67 \mathrm{fs}$. For day-to-day alignment of the probe beam along the translational stage, two mirrors are used in conjunction with two pre-fixed irises. The $R R$ reflects the incident beam at exactly $180^{\circ}$, translated in space by a few $\mathrm{mm}$ from the incident beam, depending on the incidence spot with respect to the $R R$, and size of the RR. The beam reflected from a RR mounted on a translation stage will incur "travel" as the RR slides along the stage, if the incident beam and stage travel direction are not 


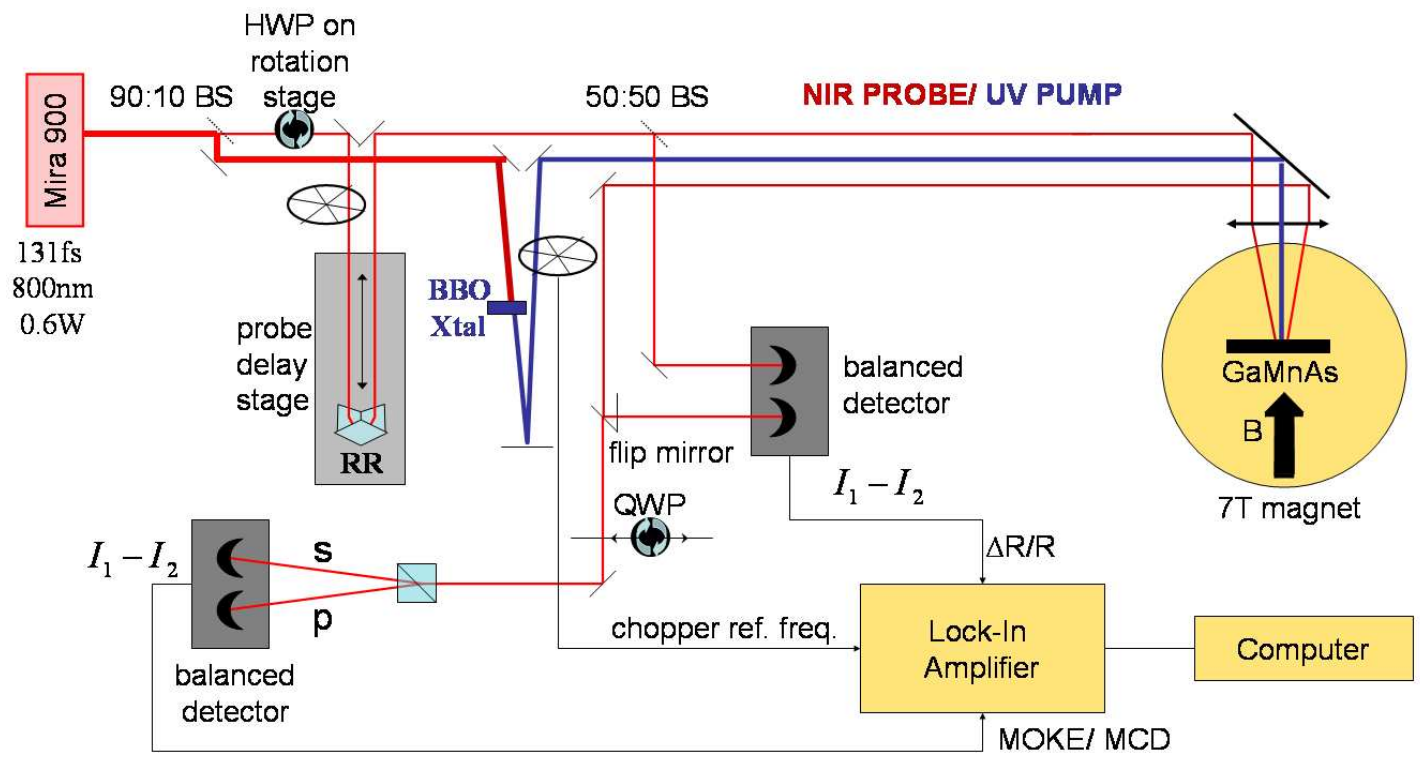

Figure 3.13: Ultrafast magnetization dynamics experimental setup, as explained in the text.

perfectly parallel. This is due to the fact that as the RR moves, the incident beam will hit different spots on it, resulting in different reflected beam positions. This displacement of the reflected beam can be horizontal or vertical, as evident for example on a paper card placed at a fixed location. To initially set the irises for the day-to-day alignment, we block about half of the retro-reflected beam with a sharp edged object (e.g., razor blade incurring extra safety measures), and send the transmitted beam onto a photodiode. We then monitor the photodiode signal while translating the stage along its entire range of motion, and adjust the direction of the incident probe beam onto the RR until any reflected beam "travel" is minimized - i.e., the photodiode signal is constant. We perform this operation for both directions the probe beam could travel by aligning the razor blade edge in each of the horizontal and the vertical directions. Once we have perfect alignment, we set the two irises in the path of the incident beam for day-to-day alignment verification. 
Daily, after aligning the probe beam with respect to the translational stage, we use the RR and a steering mirror to align the probe beam along the optical table towards the cryostat. For differential reflectivity measurements, the probe beam is further split with a 50:50 beam splitter, such that $50 \%$ is send directly to a balanced detector, while the other $50 \%$, acting as the probe beam, is sent to the sample, and in reflection, to the other arm of the balanced detector.

Meanwhile, the $90 \%$ fraction of the Mira laser output is focused onto a BBO crystal. The crystal frequency-doubles the $\approx 540 \mathrm{~mW}$ of incident NIR light, which is thus transformed into about $60 \mathrm{~mW}$ of $400 \mathrm{~nm}, 85 \mathrm{fs}$ light. This beam is used as the pump beam. To improve the signal to noise ratio, we mechanically chop the pump beam, and employ lock-in detection of the small pump-induced signals.

Both pump and probe beams are sent onto a two-mirror periscope to raise them to the appropriate height of the sample, and are focused into the cryostat with a 2 in diameter, $200 \mathrm{~mm}$ focal length lens. The pump beam is incident on the sample at normal incidence, while the probe beam makes a small, $\approx 4^{\circ}$ angle with respect to the normal, such that is reflected in a symmetrical direction with respect to the pump beam. The reflected beam then travels back through the lens and periscope, ending up in the same horizontal plane, parallel to the incident beams at $1 / 2$ in from the pump beam (see Fig. 3.14). Using a combination of steering mirrors and a flip-mirror, we can send the reflected probe beam either into a differential reflectivity $(\Delta R / R)$ setup, or into a MOKE or MCD setup.

To clarify how our measurements are made, we briefly note the principles of operation of a balanced photoreceiver, which eliminates many noise sources that can interfere 


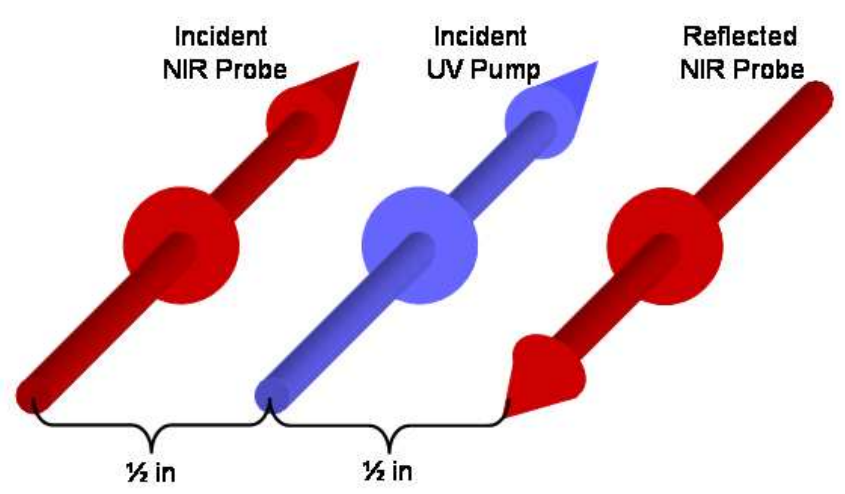

Figure 3.14: Beam geometry.

with our precision measurements. A balanced detector contains two photodiodes, with one photodiode receiving a reference beam and a second photodiode receiving a signal beam. In "autobalanced" mode our near-IR balanced detector subtracts the reference and signal photocurrents, canceling noise signals that are common to both channels. At the same time, an electronic gain compensation ensures that the reference signal is properly weighted in the subtraction, even if the average optical intensities on the two photodiodes are different or slowly time-varying due to drifts in the light source. In "traditional" balance detection mode, the photocurrents are directly subtracted, and the voltage output is directly proportional to the difference in input optical powers.

For differential reflectivity measurements, we send the probe beam reflected off the sample into the "signal" photodiode of a balanced detector, and the component directly reflecting off of the 50:50 BS into the "reference" photodiode (Fig. 3.13). In this configuration, the detector works in autobalanced mode, with the reference intensity at about twice the signal intensity. We are thus able to eliminate practically all laser intensity noise. 
For MOKE measurements, the probe beam is instead sent into a Wollaston polarizer prism, which separates the s- and p-polarized components of the probe beam. The detector is used in traditional balanced mode, and can read differences in the s and p intensities of the reflected probe induced by the pump beam. These intensities directly reflect the change of rotation angle of the probe polarization vector, which is proportional to the magnetization dynamics in the sample. MCD measurements use the same setup as for MOKE, but with a quarter wave plate (QWP) in the reflected probe path. In this case, the detector output voltage reflects ellipticity changes of the probe polarization, again proportional to the magnetization dynamics (see Appendix A). In all of these measurements, we control the magnetic field and temperature of the sample as previously described. For a description of the data acquisition codes used, please see Appendix B.

Table 3.2: Essential equipment list

\begin{tabular}{|l|l|l|}
\hline Equipment & Model and Manufacturer & Used to... \\
\hline Ar pump laser & Coherent Innova 300 & pump the Mira laser \\
Ultrafast laser & Coherent Mora 900 & produce 800nm laser pulses \\
Digital oscilloscope & Tektronix 2465B & monitor the pulses \\
Mechanical choppers & HMSLight BeamChopper 221 & improve S/N \\
Translation stage & Newport ILS 150 MVTP & delay probe w.r.t pump \\
Stage controller & Newport ESP 300 & computer control the stage \\
Balance detectors & New Focus Nirvana \# 2017 & read optical signal \\
15V Power Supply & New Focus \# 0901 & power the balance detectors \\
Rotational stage + HWP & Sigma Koki sgSP + HWP & control probe polarization \\
Stage controller & Sigma Koki PAT 001 & computer control the stage \\
Lock-in amplifier & Stanford Research Systems & read electrical signal from \\
& .. SR 830 DSP & ...balanced detectors \\
Video camera & Cohu Solid State & see incidence spot on sample \\
Power meter & Ophir AN/2 & measure beam intensity \\
\hline
\end{tabular}




\subsection{Time-resolved spectroscopy}

In this section, we detail how ultrafast time-resolved MOKE, MCD, and $\Delta R / R$ measurements are performed, and how we interpret the resulting data. As shown in Chapter 2, an appropriately spectrally-tuned, linearly polarized beam reflecting off the surface of a magnetic semiconductor will undergo rotation and ellipticity changes of its polarization vector. These effects allow us access to visualize magnetization dynamics following excitation. Moreover, $\Delta \mathrm{R} / \mathrm{R}$ can measure changes in the index of refraction of the sample, giving us insight into pump-induced charge dynamics.

\subsubsection{Magneto-optical Kerr effect measurements}

To measure the magneto-optical Kerr effect signal, the probe beam is prepared linearly polarized. We computer-control the direction of the polarization vector via a HWP mounted on a rotation stage. The reflected probe beam is sent into a Wollaston prism that separates the $\mathrm{s}^{-}$and p-polarized components into different directions. Each component is then sent into one of the two photodiodes of the balanced detector (reference and signal). In traditional balanced mode, the signal is directly proportional to the difference between the two photocurrents. To balance the detector, we block off the pump beam, chop the probe beam, feed this chopping frequency to the lock-in amplifier, and rotate the HWP establishing the direction of the probe polarization vector until the balance detector reads

0 (i.e., $\mathrm{s}$ intensity - $\mathrm{p}$ intensity $=0$ ). In this case, the polarization vector points along the $(\vec{s}+\vec{p}) / \sqrt{2}$ direction, or at $45^{\circ}$. Thus, the pre-sample probe polarization vector undergoing rotation at reflection off of the magnetized sample will be aligned exactly at $45^{\circ}$ when 
entering the Wollaston prism, and will be decomposed into two beams of exactly equal intensity.

After balancing the detector, we stop chopping the probe beam, unblock the pump beam, mechanically chop it, and reference this new frequency to the lock-in amplifier. By reading the electrical signal the balance detector outputs at different time delays between pump and probe (proportional to pump-induced changes in the rotation angle of the probe polarization), we effectively get a picture of the transient changes in the magnetic order in the sample induced by the pump.

\subsubsection{Magnetic circular dichroism measurements}

For MCD measurement, the libear polarization of the probe beam, upon reflection off of the sample, undergoes a finite retardation and becomes slightly elliptical. This elliptical polarization can be decomposed into two orthogonal linear polarizations, one with an amplitude significantly larger than the other. When passing through the QWP, if properly aligned, the two linear polarization components turn into $\sigma+$ and $\sigma-$ circular polarizations respectively, as explained in the following. Light polarized along the "fast axis" of a QWP crystal propagates faster than light polarized along the perpendicular, slow axis. Light with polarization components along both axes will emerge with the slow axis component retarded by $90^{\circ}$ with respect to the fast axis component. Therefore an input beam with a polarization oriented at $45^{\circ}$ with respect to the fast axis will emerge with circular polarization. As shown in figure 3.15, the elliptically polarized light entering the QWP can be decomposed into two linear components, each making a $\pm 45^{\circ}$ angle with the fast axis. Thus the two components emerge from the QWP as circular polarized light of opposite polarizations. 


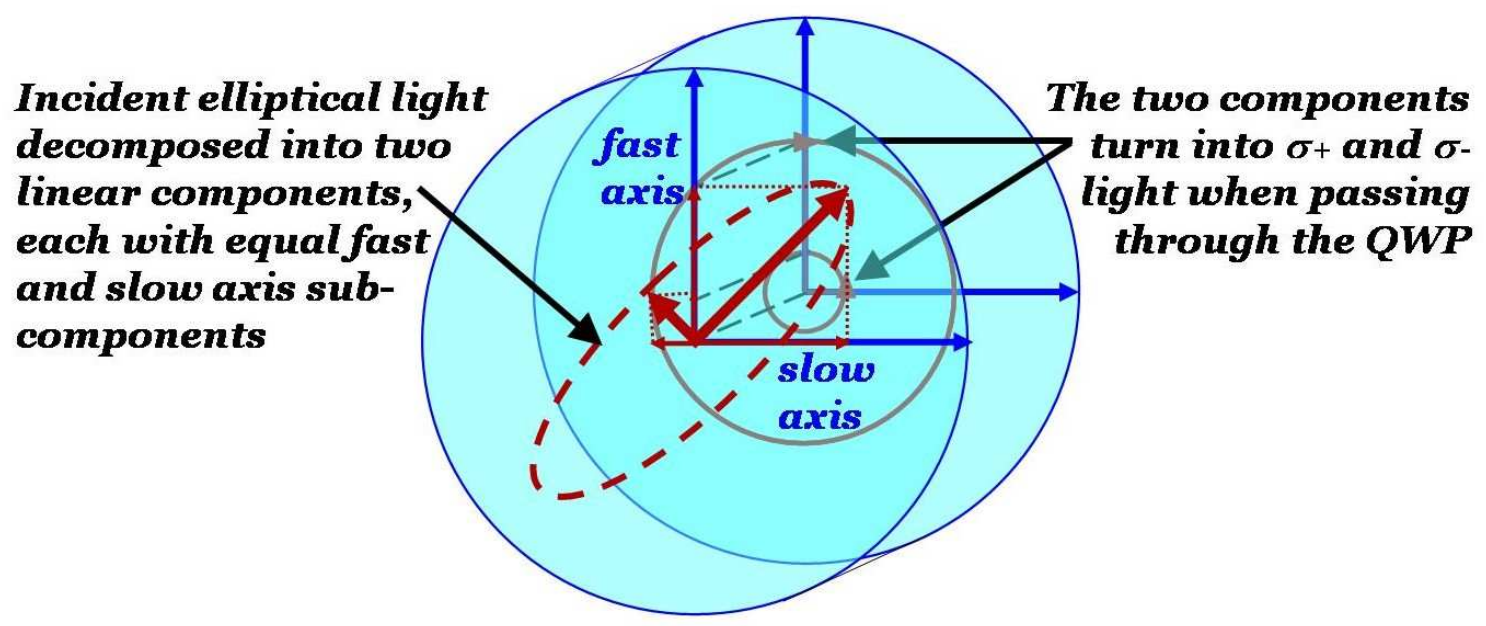

Figure 3.15: An elliptical beam passing through a QWP emerges as a combination of two circularly polarized beams.

Passing through the Wollaston prism, these circularly polarized components are again decomposed into equal intensities of $\mathrm{s}^{-}$and $\mathrm{p}$-polarized beams in two different directions. Each component is sent into one of the two photodiodes of the balanced detector (reference and signal). As in the MOKE measurement, the measured signal is directly proportional to the difference between the two photocurrents. To balance the detector chop the probe beam with the pump beam blocked. The QWP mounted on a rotation stage is then adjusted until the balanced detector output reads 0 . This condition is achieved when the reflected beam ellipsoid major axis makes a $\pm 45^{\circ}$ angle with the fast axis of the QWP. To measure the photo-induced magnetization dynamics, reflected in transient ellipticity changes of the probe polarization, we now chop the pump beam and detect the balance detector output at different time delays between pump and probe. 


\subsubsection{Differential reflectivity measurements}

In addition to the previous two techniques, which capture the magnetization dynamics, we employ two-color transient reflectivity measurements to capture the charge dynamics. Since ferromagnetism in our samples is hole-mediated, the charge dynamics is fundamental to understanding magnetization dynamics. Time-resolved differential reflectivity measurements $(\Delta \mathrm{R} / \mathrm{R})$ provide insight into charge carrier dynamics by following the transient response of the refraction index of the sample, which is connected to inter-band transitions. The NIR probe beam thus measures the bandgap response after UV photoexcitation of carriers across the gap. In this experiment, we initially block off the pump beam from hitting the sample. As explained above, we send half of the probe beam (from the 50:50 beam splitter) directly into the "Reference" photodiode of a balanced detector. The other half reflects off of the sample surface and is sent into the "Signal" photodiode of the same balanced detector. The electrical signal from this "Signal" channel corresponds to the equilibrium reflectivity $\mathrm{R}$. The balanced detector is used in autobalanced mode, with the reference channel to signal channel intensity ratio of about $2: 1$. We adjust this ratio by means of a variable optical density filter placed in the path of the reference beam.

By mechanically chopping the pump beam, when the pump is unblocked, the lockin amplifier now detects the pump-induced changes in the reflectivity of the probe beam. The ratio of the transient electrical signal read by the autobalanced detector to the previously

determined reflectivity $\mathrm{R}$ gives the transient reflectivity change $\Delta \mathrm{R} / \mathrm{R}$ we are interested in, in effect measuring pump-induced transient charge dynamics. 


\subsection{Experimental parameters and signal analysis}

We now describe relevant calculations necessary for the data analysis (spot size measurement, absorption depths, excited carrier density), and the method of extracting the MOKE, MCD and $\Delta \mathrm{R} / \mathrm{R}$ signals.

\subsubsection{Excitation density}

In order to estimate the number of carriers created by the laser pulse in our experiments, we need to estimate the laser focus diameter at the sample. To do so, we image the sample using a video camera, and estimate the laser spot size relative to the sample size. Since we can measure the real size of the sample outside the cryostat, this yields an estimate of the laser spot diameter, $2 \mathrm{r}_{0} \approx 50 \mu \mathrm{m}$.

We must also determine the absorption coefficient $\alpha(\omega)$ of GaMnAs, which we approximate to that of GaAs for the two wavelengths of interest. This yields the respective absorption depths [85, 14]:

$$
\begin{gathered}
\alpha_{(800 \mathrm{~nm})} \approx 1 \times 10^{4} \mathrm{~cm}^{-1} \\
\delta_{(800 \mathrm{~nm})} \approx 1000 \mathrm{~nm} \\
\alpha_{(400 \mathrm{~nm})} \approx 7 \times 10^{5} \mathrm{~cm}^{-1} \\
\delta_{(400 \mathrm{~nm})} \approx 14 \mathrm{~nm}
\end{gathered}
$$

We can now calculate the density of carriers excited by the laser during our experiments as follows. For almost all of our samples (A, C, D, and E), the film thickness is much larger than the $400 \mathrm{~nm}$ absorption depth, and much smaller than the $800 \mathrm{~nm}$ absorption 
depth. That means that it is practical to estimate that all of the UV light is absorbed by the sample (neglecting any reflection), while practically almost all NIR light is reflected (or absorbed in the sample substrate). The average number of excited carriers created by the UV pulse, per unit area, is:

$$
N=\frac{1}{f} \int \frac{I(\omega)}{\hbar \omega} d \omega \mathrm{cm}^{-2} \approx \frac{1}{f} \frac{I_{T}}{\hbar \bar{\omega}} \mathrm{cm}^{-2}
$$

where $\mathrm{f}$ is the laser repetition rate $\left(80 \times 10^{6} \mathrm{pulses} / \mathrm{sec}\right)$ and $I_{T}$ is the average beam intensity. While the laser beam intensity is not uniform in the radial direction (having a Gaussian profile), we approximate the intensity $I_{T}$ by a constant average intensity within a radius $r_{0}$ :

$$
I_{T}=\frac{P_{T}}{\pi r_{0}^{2}} \frac{W}{\mathrm{~cm}^{2}}
$$

where $P_{T}$ is the total beam power, as measured by a power meter, and $2 r_{0}=50 \mu \mathrm{m}$. Combining the above, we obtain the total excited carrier sheet density:

$$
N=\frac{1}{f} \frac{P_{T}}{\pi r_{0}^{2}} \frac{1}{\hbar \omega}=\frac{1}{80 M H z} \frac{30 m W}{\pi(25 \mu m)^{2}} \frac{1}{3.1 e V} \approx 3.85 \times 10^{13} \mathrm{~cm}^{-2}
$$

For $P_{T}=30 \mathrm{~mW}$, this represents the average sheet density of carriers excited by one $\mathrm{UV}$ pulse. Since we know the light is absorbed by the sample according to

$$
I(x)=I_{0} e^{-\alpha x}
$$

we can set the spatial density of excited carriers to follow the exponential intensity decay, such that the total sheet density equals $\mathrm{N}$ as previously calculated:

$$
N=\int_{0}^{\infty} n_{0} e^{-\alpha x} d x=\frac{n_{0}}{\alpha_{(400 n m)}}=n_{0} \delta_{(400 n m)}
$$


Thus, for most of our samples, the density of excited carriers varies exponentially from $n_{0}$ to 0 into the sample, where:

$$
n_{0}=N_{(30 m W)} \alpha_{(400 n m)} \approx 2.7 \times 10^{19} \frac{\text { carriers }}{\mathrm{cm}^{3}}
$$

for a $30 \mathrm{~mW}$ input UV beam.

\subsubsection{Signal analysis}

\section{MOKE}

For MOKE signals, the lock-in amplifier reads the transient pump-induced changes received from the balanced detector. Consider the polarization schematics in figure 3.16. Initially, the detector is balanced, equivalent to $\alpha=45^{\circ}$. When the pump hits the sample and induces magnetization dynamics, the probe polarization vector rotates to a new angle $\beta(t)$. In polar MOKE, $\Delta M_{z}(t) \propto(\beta(t)-\alpha)$, so we calculate the change in angle $\beta(t)-\alpha$ as a function of intensities measured by the balanced detector:

$$
\begin{gathered}
\beta-\alpha \approx \sin (\beta-\alpha)=\sin \beta \cos \alpha-\cos \beta \sin \alpha \\
\beta-\alpha \approx \frac{I_{p}+\Delta I_{p}}{I} \frac{\sqrt{2}}{2}-\frac{I_{s}+\Delta I_{s}}{I} \frac{\sqrt{2}}{2}
\end{gathered}
$$

When the detector is initially balanced, the two channels receive equal intensity beams $I_{s}=I_{p}=I / \sqrt{2}$. Thus, we obtain:

$$
\Delta \theta_{M O K E}(t)=\beta-\alpha \approx \frac{\Delta\left(I_{p}-I_{s}\right)_{(t)}}{2 I_{p}}
$$

The lock-in amplifier reads exactly the change in (s channel - p channel) difference. Therefore, it follows that to obtain the MOKE signal (in radians) we must divide the lock-in signal 


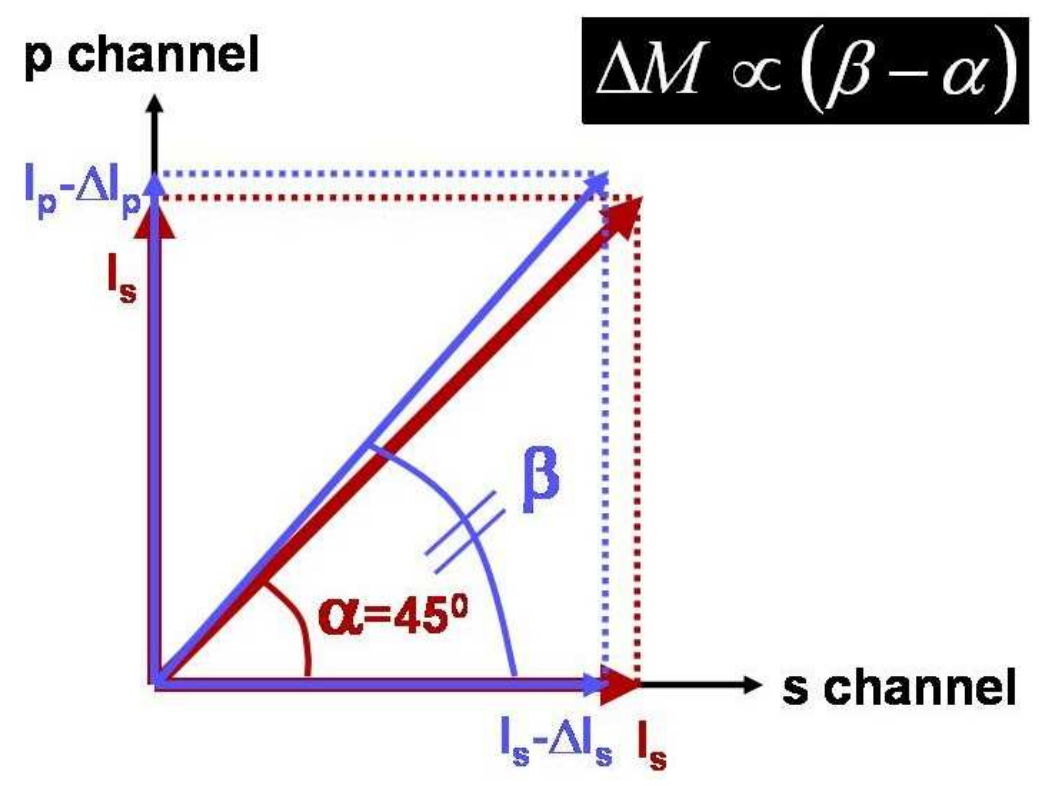

Figure 3.16: The $45^{\circ}$ reflected probe polarization is split into equal $\mathrm{s}$ and $\mathrm{p}$ components into each of the two balanced detector channels. Magnetization dynamics induce rotation of the polarization, which translates into a net detector signal, proportional to the angle of rotation and change in magnetization.

$\Delta\left(I_{p}-I_{s}\right)$ by twice the balanced detector signal per channel, $2 I_{p}$. Typically $\Delta \theta_{M O K E}$ is on the order of $1-100 \mu \mathrm{rad}$, such that the above small angle approximation is valid.

\section{MCD}

For MCD signals, the lock-in amplifier also reads the transient pump-induced changes received from the balanced detector. The probe polarization, however, is a slightly elliptical p polarization when it impinges on the QWP. The ellipticity, defined as ratio of the minor axis to the major axis of the ellipse, is on the order of $10^{-3}$ and is proportional to the sample magnetization. As shown in figure 3.15, each of the two linear components of this ellipse turns into a circularly polarized beam when emerging from the QWP. We sketch this phenomenon in figure 3.17, where we depict the probe polarization pre-QWP, 
post-QWP, and as it arrives at the balanced detector, after being separated into s and $\mathrm{p}$ components by the Wollaston prism.

As we stated earlier, the ellipticity of the reflected probe beam polarization is proportional to the magnetization in the sample, i. e.:

$$
\eta=\frac{b}{a} \propto M
$$

To measure magnetization dynamics, we aim to measure transient changes in ellipticity. Since we can assume that the intensity of the reflected beam is pretty much constant, it follows that:

$$
\begin{gathered}
a^{2}+b^{2}=\text { const }=>\Delta a=-\frac{b}{a} \Delta b<<\Delta b \\
\Delta M \propto \Delta \eta \approx \frac{\Delta b}{a}
\end{gathered}
$$

Now, the balanced detector measures pump-induced changes in the difference between the signals in the $\mathrm{p}$ channel and the s channel as marked in figure 3.17 , i.e., $\Delta\left(I_{p}-\right.$ $\left.I_{s}\right)$. The changes in magnetization are much smaller than the magnetization amplitude, i.e., changes per channel are much smaller than signal per channel, hence we can write:

$$
\Delta\left(I_{p}-I_{s}\right)=\Delta\left[\frac{1}{2}\left(\frac{(a+b)^{2}}{2}-\frac{(a-b)^{2}}{2}\right)\right]=\Delta(a b)
$$

which, because $\Delta a<<\Delta b$, turns into:

$$
\Delta\left(I_{p}-I_{s}\right) \approx a \Delta b
$$

The signal per channel, for $b<<a$, is $I_{s} \approx I_{p} \approx a^{2} / 4$. It follows that to obtain the MCD signal, i.e., transient changes of the probe polarization ellipticity, we need to divide the lock-in amplifier signal $\Delta\left(I_{p}-I_{s}\right)$ by four times the signal per channel:

$$
\Delta \eta_{M C D}(t)=\frac{\Delta b}{a}=\frac{\Delta\left(I_{p}-I_{s}\right)_{(t)}}{4 I_{p}}
$$



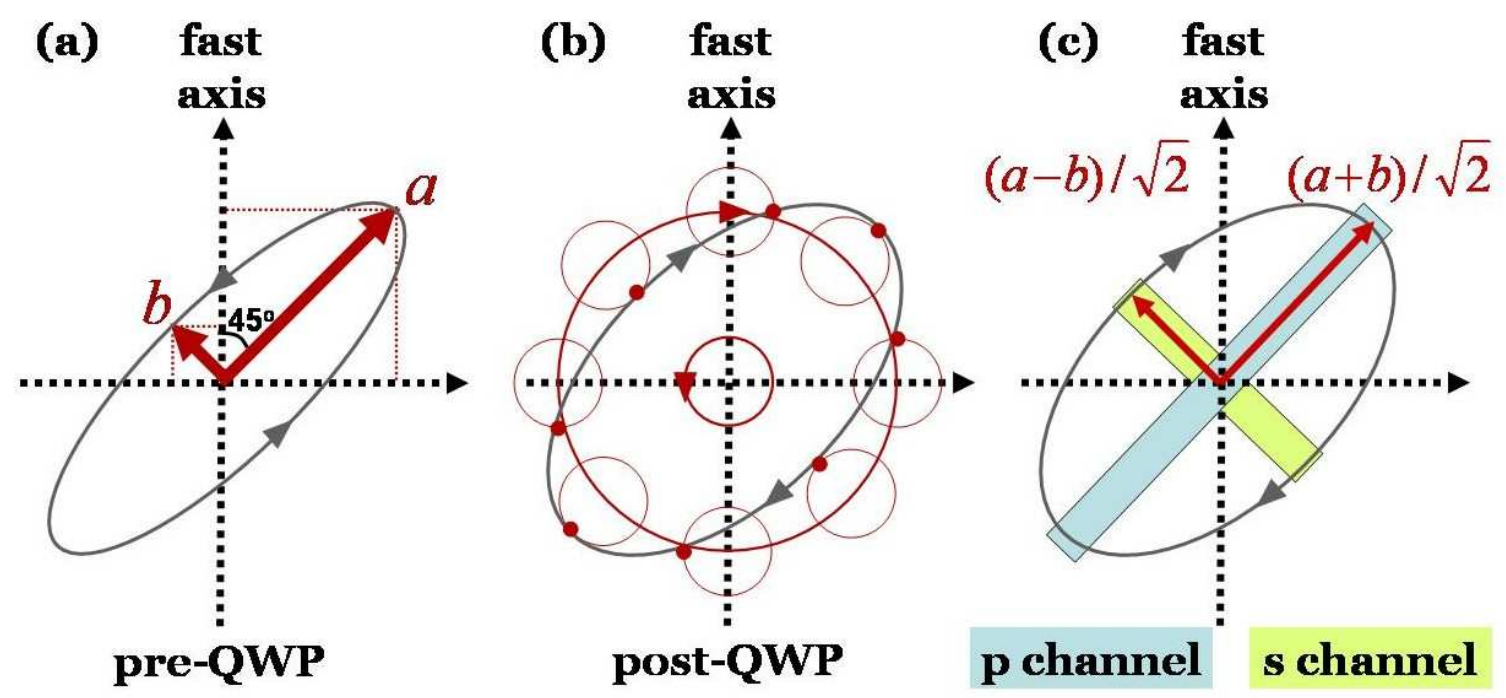

Figure 3.17: (a) The slightly elliptical p probe polarization is composed of two linear polarization components of amplitude $\mathbf{a}$ and $\mathbf{b}$, out of phase by $90^{\circ}$. The major axis subtends a $45^{\circ}$ angle with the fast axis of the QWP. (b) Post-QWP, each linear component turns into a circular polarization, of opposite helicity, which together form an almost perfectly circular polarization (ellipticity exaggerated in schematics). (c) Light arrives at the balanced detector split into $\mathrm{s}$ and $\mathrm{p}$ components, almost equal to each other (within the detection precision). Magnetization dynamics induce changes of the ellipticity, which translates into a net detector signal. 


\section{Differential reflectivity}

$\Delta \mathrm{R} / \mathrm{R}$ measurements are generally simpler to interpret. The reflected probe beam intensity $\mathrm{R}$ is recorded as the intensity of light in the balanced detector "signal" channel. In autobalanced mode, transient changes of intensity of the signal channel are recorded through the lock-in amplifier as $\Delta \mathrm{R}$. It follows that to measure differential reflectivity, one needs to divide the transient changes of the signal channel by the initial signal channel intensity:

$$
\frac{\Delta R_{(t)}}{R}=\frac{\Delta I_{\text {sig }}(t)}{I_{\text {sig }}}
$$




\subsection{Conclusion}

In this chapter, we described the experimental setup, including the samples, ultrafast laser system, magneto-optical cryostat, and experimental layout, along with the time-resolved spectroscopy techniques and signal evaluation. We included alignment procedures, control parameters, and details on how MOKE, MCD, and $\Delta R / R$ signals are interpreted to extract the physically relevant information. It turns out that if we employ a suitable combination of polar MOKE and different probe polarization $\Delta \mathrm{R} / \mathrm{R}$ measurements (see Appendix C), we gain full access to the dynamics of the $3 \mathrm{D}$ magnetization vector. Thus, we can visualize its 3D trajectory as it evolves in time (Fig. 3.18):

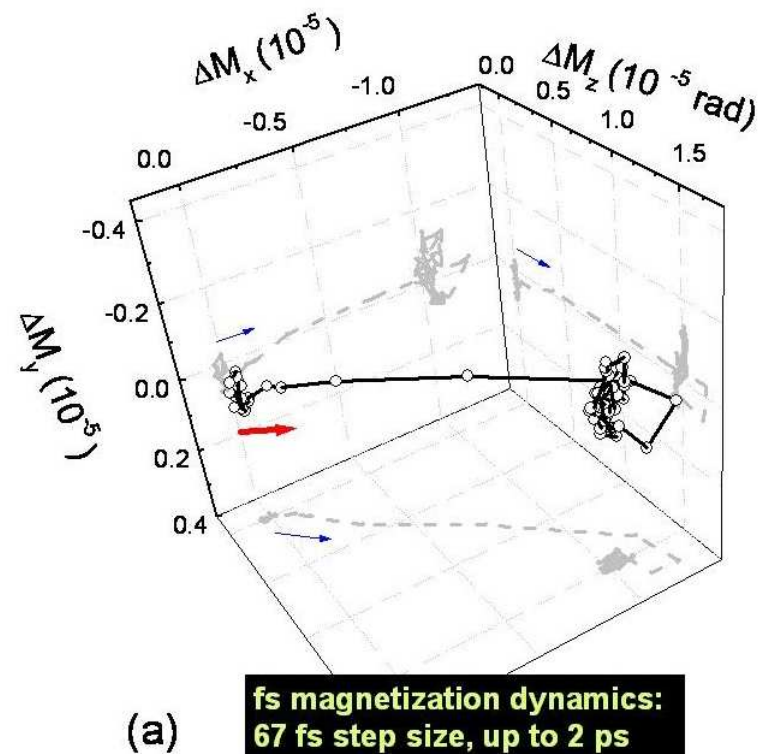

(a) 67 fs step size, up to 2 ps

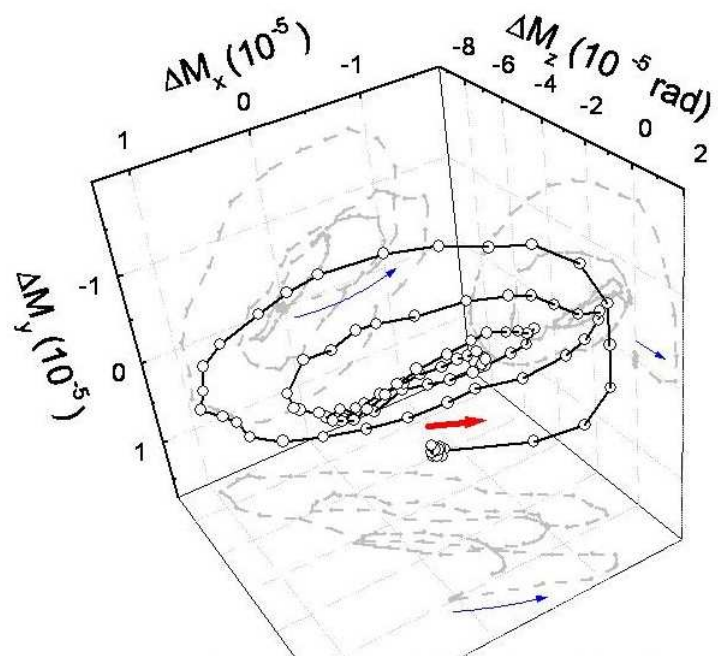

ps magnetization dynamics:

(b) 9.3 ps step size, up to $1 \mathrm{~ns}$

Figure 3.18: Three dimensional trajectory of magnetization vector transient changes induced by the pump pulse, evolving on the (a) fs and (b) ps timescales.

Armed with these techniques, we are ready to proceed with presenting our results on ultrafast magnetization dynamics in ferromagnetic semiconductors. 


\section{Chapter 4}

\section{Ultrafast Ferromagnetic Phase}

\section{Enhancement}

\subsection{Outline}

We investigate the possibility to enhance the ferromagnetic phase in GaMnAs via time-resolved, ultra-violet pump, near-infrared probe MOKE spectroscopy. Closely related to this enhancement of the FM phase, we search for a paramagnetic to ferromagnetic phase transition. In our experiments, the time evolution of the magnetization vector amplitude is monitored after irradiation with ultrashort UV laser pulses. The amplitude of the spontaneous magnetization acts as a measure of the FM interaction strength. 


\subsection{Magnetization amplitude dynamics in MBE-grown}

\section{$(\mathrm{Ga}, \mathrm{Mn}) \mathrm{As}$}

The experimental setup and sample details were described in previous chapters. We present here the experimental results obtained by two color, transient polar MOKE spectroscopy on sample A [94]. We first study charge dynamics in our sample by differential reflectivity. We will find that after photo-generation of electron-hole pairs, the electrons are quickly trapped by deep defect states. The holes, on the other hand, are long lived $(\tau \sim 2 \mathrm{~ns})$, participating in the mediation of the FM interaction and strengthening it. At temperatures below $\mathrm{T}_{C}$, the spontaneous magnetization lies in the sample plane, along an easy axis direction. In our polar MOKE experiments, in order to monitor amplitude dynamics of the magnetization vector, we apply an external magnetic field of $1 \mathrm{~T}$ perpendicular to the sample plane (i.e., along the $\hat{z}$ axis) which is strong enough to keep the magnetization aligned along it at all times. Thus, measuring $\Delta M_{z}(t)$ provides the amplitude dynamics of the magnetization vector $\Delta M(t)$. Our data clearly show photoinduced magnetization enhancement on a 100 ps time scale, after initial sub-picosecond demagnetization. As we will show, the dynamic magnetization enhancement exhibits a maximum below $\mathrm{T}_{C}$ and dominates the demagnetization component when approaching $\mathrm{T}_{C}$. Our analysis and theoretical simulations based on the $\mathrm{H}_{p-d}$ interaction between photoexcited holes and $\mathrm{Mn}$ spins explain the salient features of the experiment showing, in particular, a correlationinduced peak around $20 \mathrm{~K}$ and a transient increase in $\mathrm{T}_{C}$. In order to reveal signatures of a transient para- to FM phase transition, we present magnetization dynamics near the transition temperature $\mathrm{T}_{C}$. 


\subsubsection{Charge dynamics}

Typical room temperature data showing ultrafast carrier dynamics in ferromagnetic GaMnAs is presented in figures 4.1 and 4.2. A complex carrier decay dynamics is observed. First, the initial change in the differential reflectivity is negative for times less than 1 ps after photo-excitation (Fig. 4.1). Second, a rapid reflectivity rise is observed, leading to a sign change. Next, a build-up of the reflectivity signal is observed. Finally, the signal shows a very slow decay towards zero (Fig. 4.2, several hundred ps). This behavior agrees with previous experiments on III-V DMS [96].

The fast $(\sim 2 \mathrm{ps})$ decay of the initial negative signal reflects a unique feature of low-temperature MBE growth, and can be attributed to the ultrafast trapping of electrons (by $\mathrm{As}_{G a}$ antisite defects) and holes (by Ga vacancies), by mid-bandgap states due to defects. Because of these trapping processes, the free carrier lifetimes in III-V ferromagnetic semiconductors at low temperature are less than 1 ps.

The initial sharp dip in the differential reflectivity results from free carrier Drude absorption by the hot photo-generated carriers. The photo-generated hot carriers relax to quasi-equilibrium distributions through emission of LO phonons and the ultrafast trapping

of electrons (by $\mathrm{As}_{G a}$ antisite defects) and holes (by Ga vacancies). This alters the dielectric function of the heterostructure through changes in the electron and hole distribution functions, and gives rise to the sharp increase and sign change in the differential reflectivity seen in figure 4.1. The long decay time after the rise in reflectivity suggests a slow recombination of the trapped carriers, which finally vacates the carrier traps and causes the differential reflectivity to return to zero. Part of this slow decay in the differential reflectivity can be 


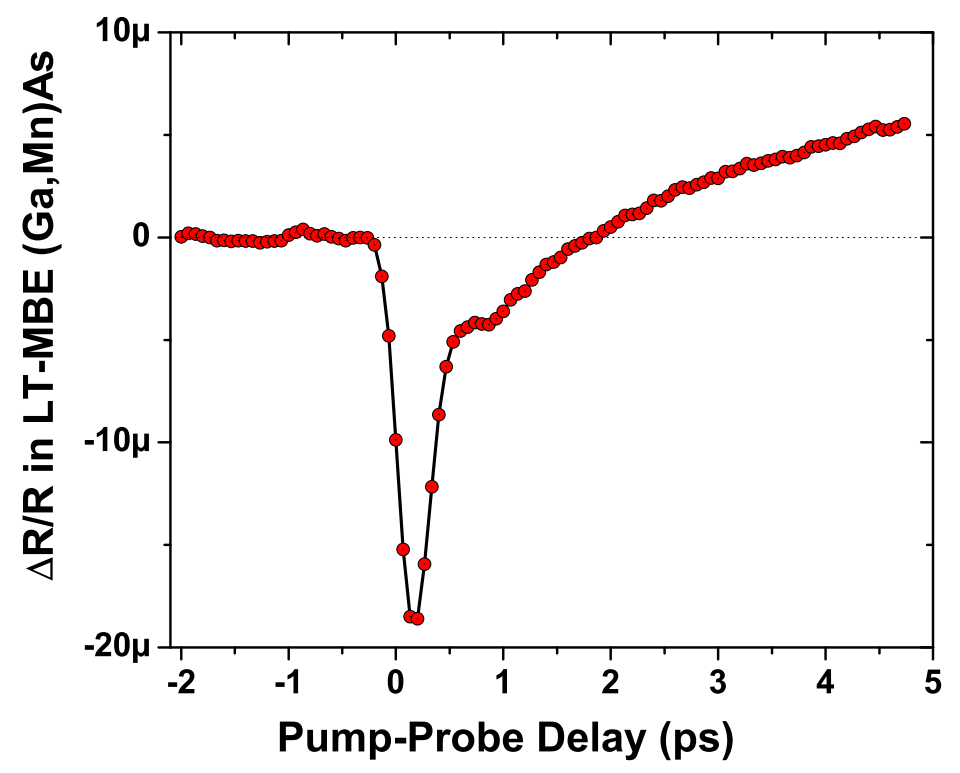

Figure 4.1: Above $\mathrm{T}_{C}$ differential reflectivity measurements in LT-MBE (Ga,Mn)As, (sample $\mathrm{A}$ ) at the ps timescale. $\Delta \mathrm{R} / \mathrm{R}$ is initially negative, due to initial absorption, followed by a rapid reflectivity rise due to ultrafast trapping of electrons.

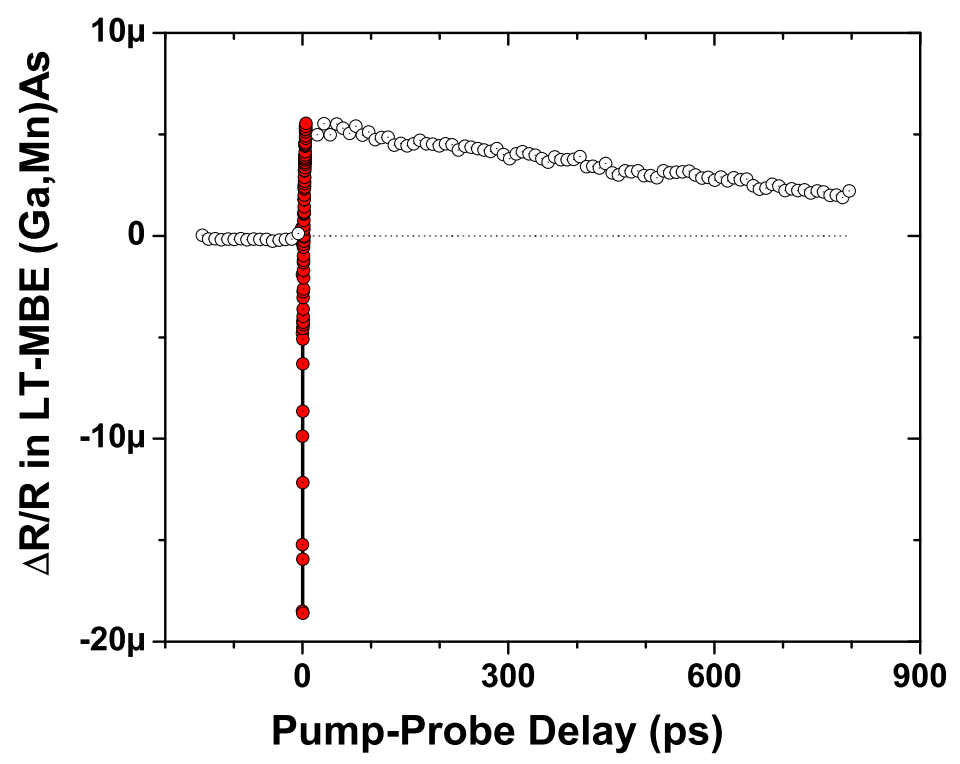

Figure 4.2: Above $\mathrm{T}_{C}$ reflectivity changes $\Delta \mathrm{R} / \mathrm{R}(\mathrm{t})$ on the ns timescale in sample $\mathrm{A}$. 
seen in the long time signal in the differential reflectivity in figure 4.2.

\subsubsection{Ferromagnetic phase enhancement}

We will show that our measurements involving UV pump - NIR probe MOKE spectroscopy demonstrate a clear enhancement of magnetization and increase of $\mathrm{T}_{C}$, as illustrated in figure 4.3(a). The experimental setup is as described in Chapter 3, with the $3.1 \mathrm{eV}$ pump beam linearly polarized. Peak fluences are $\sim 10 \mu \mathrm{J} / \mathrm{cm}^{2}$. As illustrated in figure 4.3(b), femtosecond pump pulses create a large density of holes in the valence band of GaMnAs. The low pump peak fluences and the high pump photon energy minimize spurious effects such as two-photon absorption and pump scattering. Additionally, the "magnetic origin" of the transient MOKE response is confirmed by separate measurements showing the overlap of the pump-induced rotation and ellipticity through the entire measured temporal range (see Appendix A) [45].

A typical temporal profile of transient MOKE changes $\Delta \theta_{K}(t) \propto \Delta M(t)$ at $70 \mathrm{~K}$ is shown in figure 4.3(c), with a field of $1 \mathrm{~T}$ perpendicular to the sample surface to align the magnetization along it. Two mutually competing dynamic magnetization processes are observed: an initial sub-picosecond demagnetization $\left(\Delta \theta_{K}<0\right)$, followed by a distinct magnetization rise on a $100 \mathrm{ps}$ time scale $\left(\Delta \theta_{K}>0\right)$. The two processes show different temperature dependences, as shown in figure $4.4(\mathrm{a}-\mathrm{b})$. At elevated lattice temperature, the 200 fs demagnetization components (inset of Fig. 4.4(b)) quickly diminish and nearly disappear above $\mathrm{T}_{C}$. This is also seen in the $600 \mathrm{fs}$ and 3 ps traces in figure 4.4(c). More intriguingly, an enhancement of the transient magnetization begins to dominate the demagnetization component at high temperatures. The temporal traces of $\Delta \theta_{K}$ at temperatures from 40 

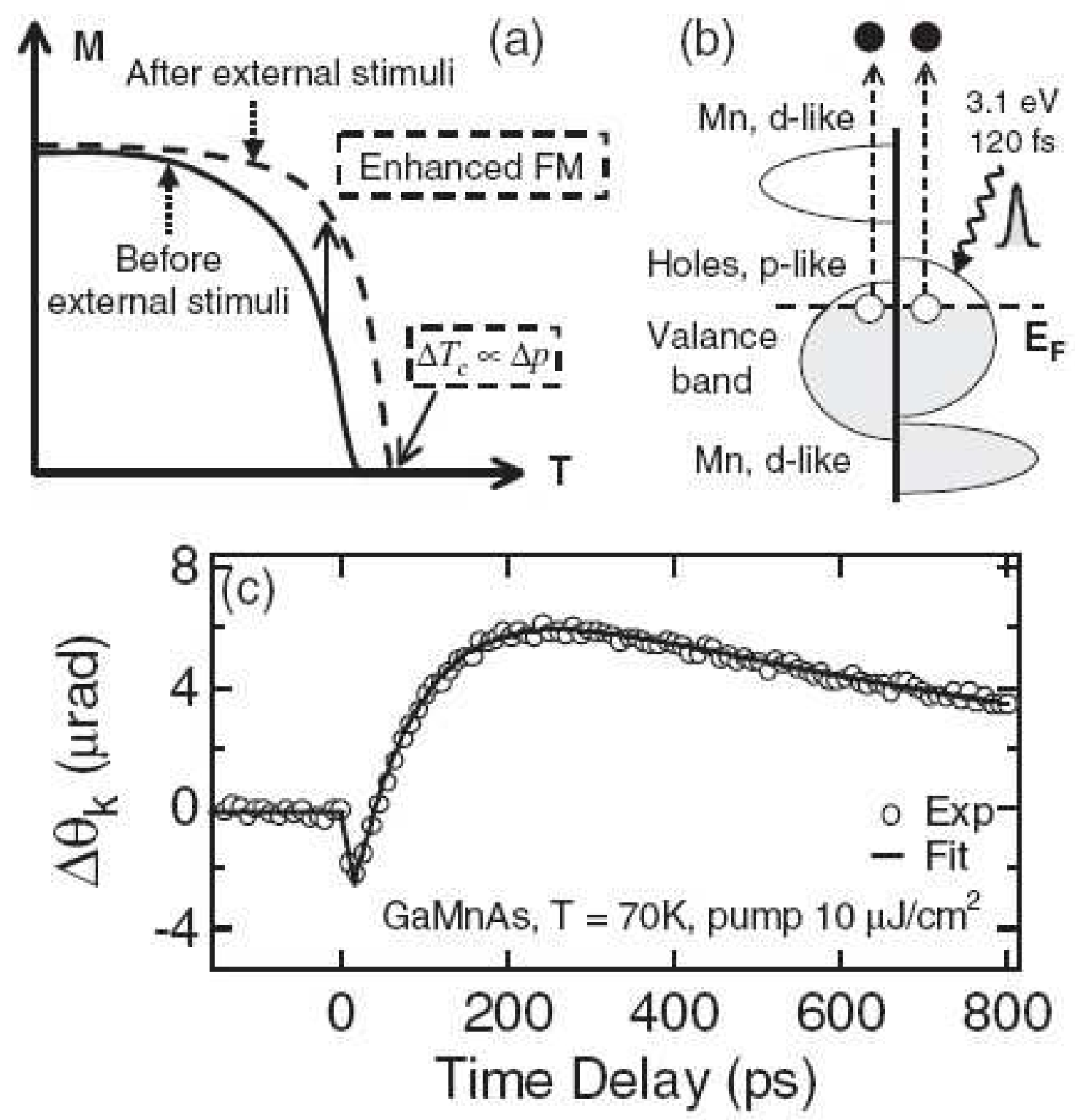

Figure 4.3: (a) Illustration of hole-density-tuning effects via external stimuli in III-V DMS seen in the static experiments $[47,61] . \Delta \mathrm{p}$ is the hole density change. (b) Schematic diagram of the spin-dependent density of states in GaMnAs. Femtosecond pump pulses create a transient population of holes in the valence band. (c) Time-resolved MOKE dynamics for $\mathrm{T}=70 \mathrm{~K}$ and $1 \mathrm{~T}$ external magnetic field. A transient enhancement of magnetization, with $\sim 100$ ps rise time, is clearly seen after initial fast demagnetization. 
to $70 \mathrm{~K}$ in figure 4.4(b) clearly show that the net magnetization changes become positive at long time delays. Figure 4.4(c) summarizes the photoinduced magnetization changes at 240 ps revealing $\Delta \theta_{K}>0$ above $40 \mathrm{~K}$. The photoinduced magnetization persists above $\mathrm{T}_{C}$ - as is clearly visible in the $80 \mathrm{~K}$ trace (Fig. 4.4(b)) - and gradually vanishes at higher temperatures.

MOKE signals, measured with the $1.55 \mathrm{eV}$ probe, arise from the macroscopic magnetization $\mathrm{M}$ - average localized Mn spins - through the coupling of the spin-split electronic states near the band edge. The background carrier spin contribution to $\mathrm{M}$ is negligible, and photoexcited transient carriers are not spin polarized since the pump contains no net angular momentum. Thus the positive MOKE signals, rising on a $100 \mathrm{ps}$ time scale, clearly indicate an ultrafast alignment of Mn spins and an enhancement of ferromagnetic order. Our results reveal the ultrafast time scale of this process, not accessible in previous static measurements $[47,61]$.

We attribute the observed ultrafast photoenhanced ferromagnetism to the transient hole-Mn interaction via the $\mathrm{H}_{p d}$ exchange. At early pump-probe delays $(\Delta \mathrm{t} \sim 0 \mathrm{fs})$, the ultrafast laser pulses generate a non-equilibrium distribution of hot spin-unpolarized electron-hole pairs in GaMnAs under a finite external H field. During the first picosecond $(\Delta t<1 \mathrm{ps})$, the photoexcited hot holes will experience efficient spin-flip scattering with the localized Mn moments, which will manifest as the sub-picosecond demagnetization component. This results from the off-diagonal elements of the exchange Hamiltonian:

$$
H_{p d}=J_{p d} \overline{\mathbf{S}}^{M n} \overline{\mathbf{s}}^{h}=J_{p d} \overline{\mathbf{S}}_{z}^{M n} \overline{\mathbf{s}}_{z}^{h}+\left[J_{p d} \overline{\mathbf{S}}_{+}^{M n} \overline{\mathbf{S}}_{-}^{h}+J_{p d} \overline{\mathbf{S}}_{-}^{M n} \overline{\mathbf{s}}_{+}^{h}\right]
$$

which cause the spin polarization of the Mn ions to transfer to the holes within several 

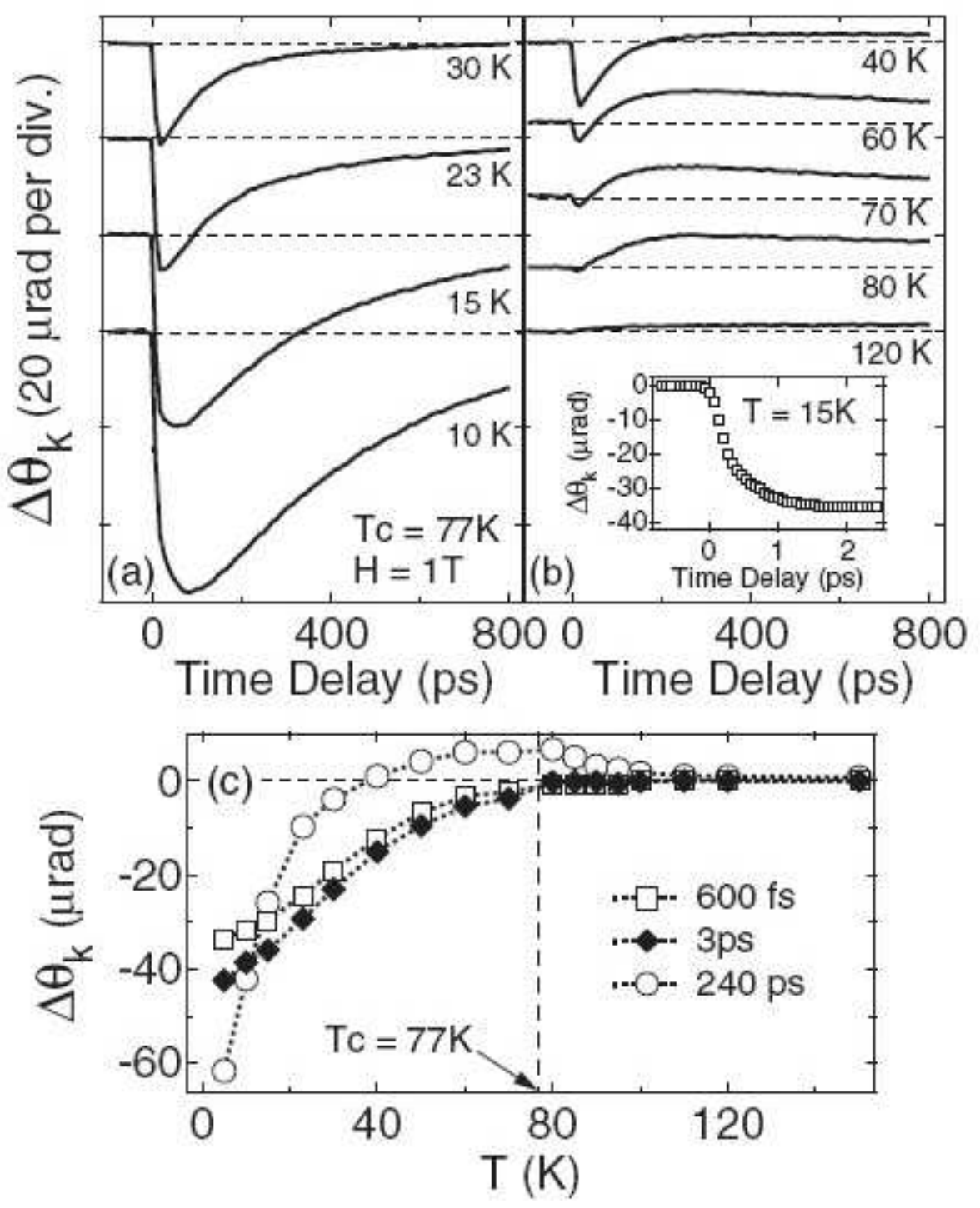

Figure 4.4: (a),(b) Temporal traces of photoinduced magnetization changes at different temperatures. All traces are intentionally offset for clarity. Inset: the first 2 ps demagnetization dynamics at $15 \mathrm{~K}$. (c) Temperature dependence of magnetization changes at different time delays: $600 \mathrm{fs}$ (squares), 3 ps (diamonds) and $240 \mathrm{ps}$ (circles), respectively. 
hundred femtoseconds, similar to the femtosecond demagnetization first reported in InMnAs [97]. Meanwhile, the hot hole distribution quickly loses its energy via carrier-phonon scattering (optical phonon energy $\sim 36 \mathrm{meV}$ ), resulting in a rapid termination of demagnetization (within the first picosecond). At longer pump-probe delays of $\Delta t>1 \mathrm{ps}$, the photoexcited, thermalized holes settle down in the spin-split bands at the center of the Brillouin zone. Therefore, they can now participate mediating the ferromagnetic order. These extra holes enhance the Mn-Mn exchange correlation and polarize the Mn spins via the mean field (diagonal) elements of the exchange Hamiltonian $H_{p d}^{m f}=J_{p d} \overline{\mathbf{S}}_{z}^{M n} \overline{\mathbf{s}}_{z}^{h}$, thereby increasing the macroscopic magnetization.

In order to elucidate the salient features of the photoenhanced ferromagnetism, we decompose the transient MOKE changes shown in figure 4.4 into demagnetization $\left(-\Delta \theta_{K}^{d}\right)$ and enhanced magnetization $\left(\Delta \theta_{K}^{m}\right)$ components, based on their different time scales. The temporal profile of $\Delta \theta_{K}$ is well described by:

$$
\Delta \theta_{K}=\Delta \theta_{K}^{d} \exp \left(-t / \tau_{d}\right)+\Delta \theta_{K}^{m}\left[1-\exp \left(-t / \tau_{m}\right)\right] \exp \left(-t / \tau_{c}\right)
$$

Here $\Delta \theta_{K}^{d}$ and $\tau_{d}$ in the first term are the magnitude of demagnetization and the recovery time determined by the slow heat diffusion process, respectively. In the second term, $\Delta \theta_{K}^{m}$ and $\tau_{m}$ are the magnitude and buildup time of the enhanced magnetization component, respectively, while $\tau_{c}$ accounts for the final decay of the magnetization enhancement via hole diffusion and recombination. The time constant $\tau_{c}$ is on the order of a few nanoseconds, as seen in the decay of the positive MOKE signal. As an example, the thick line in figure 4.3(c) represents the fit of the MOKE dynamics at $70 \mathrm{~K}$.

Figure 4.5 plots the temperature dependence of $-\Delta \theta_{K}^{d}$ and $\Delta \theta_{K}^{m}$. The $-\Delta \theta_{K}^{d}$ pro- 
file resembles the static magnetization curve of the sample (inset of Fig. 4.5(a)), exhibiting a strong deviation from the classical mean-field convex shape. This non-classical behavior of magnetization arises from the existence of two strongly interacting spin ensembles, Mn and holes, as discussed in [19]. More intriguingly, the magnetization enhancement $\Delta \theta_{K}^{m}$ in figure 4.5 (b) shows a distinctly different temperature profile, with a peak of $\sim 0.5 \%$ of the saturation magnetization $\mathrm{M}_{0}$ around $20 \mathrm{~K}$ (static $\theta_{K}$ at $5 \mathrm{~K} \sim 4 \mathrm{mrad}$ ) and a prolonged tail extended to $\sim 120 \mathrm{~K}$. The extended profile beyond $\mathrm{T}_{C}$ is expected as the combined effect of an applied external field of $1 \mathrm{~T}$ and of hole-enhanced magnetic susceptibility in the paramagnetic state. The most salient feature of the photoenhanced ferromagnetism is the peak near $20 \mathrm{~K}$, which is a manifestation of the Mn-hole correlation $\mathrm{H}_{p d}$.

Qualitatively, we explain this by the different characteristic temperature dependence of the molecular fields of holes and Mn spins, respectively, as illustrated in figure 4.6. The ferromagnetic molecular field acting on the Mn ions (holes) is determined by the average spin polarizations of the holes (Mn ions) via the Mn-hole exchange coupling $J_{p d}$. The effective field acting on the holes is much larger than that acting on the Mn ions, because of the large density of Mn ions compared to the holes, and larger spin. As a consequence, the hole polarization will remain saturated at a temperature $\mathrm{T}_{h}$ much higher than that needed for saturating the Mn magnetization $\left(T_{M} \sim 0 \mathrm{~K}\right)$. As the lattice temperature increases above $\mathrm{T}_{M n}$ but less than $\mathrm{T}_{h}$, the Mn spins with partial alignment begin to be efficiently polarized via photoexcited holes with near-unity magnetization. However, as the lattice temperature rises above $\mathrm{T}_{h}$, because of the reduced hole polarization, the efficiency of the magnetization enhancement process quickly drops, resulting in a magnetization enhancement maximum 


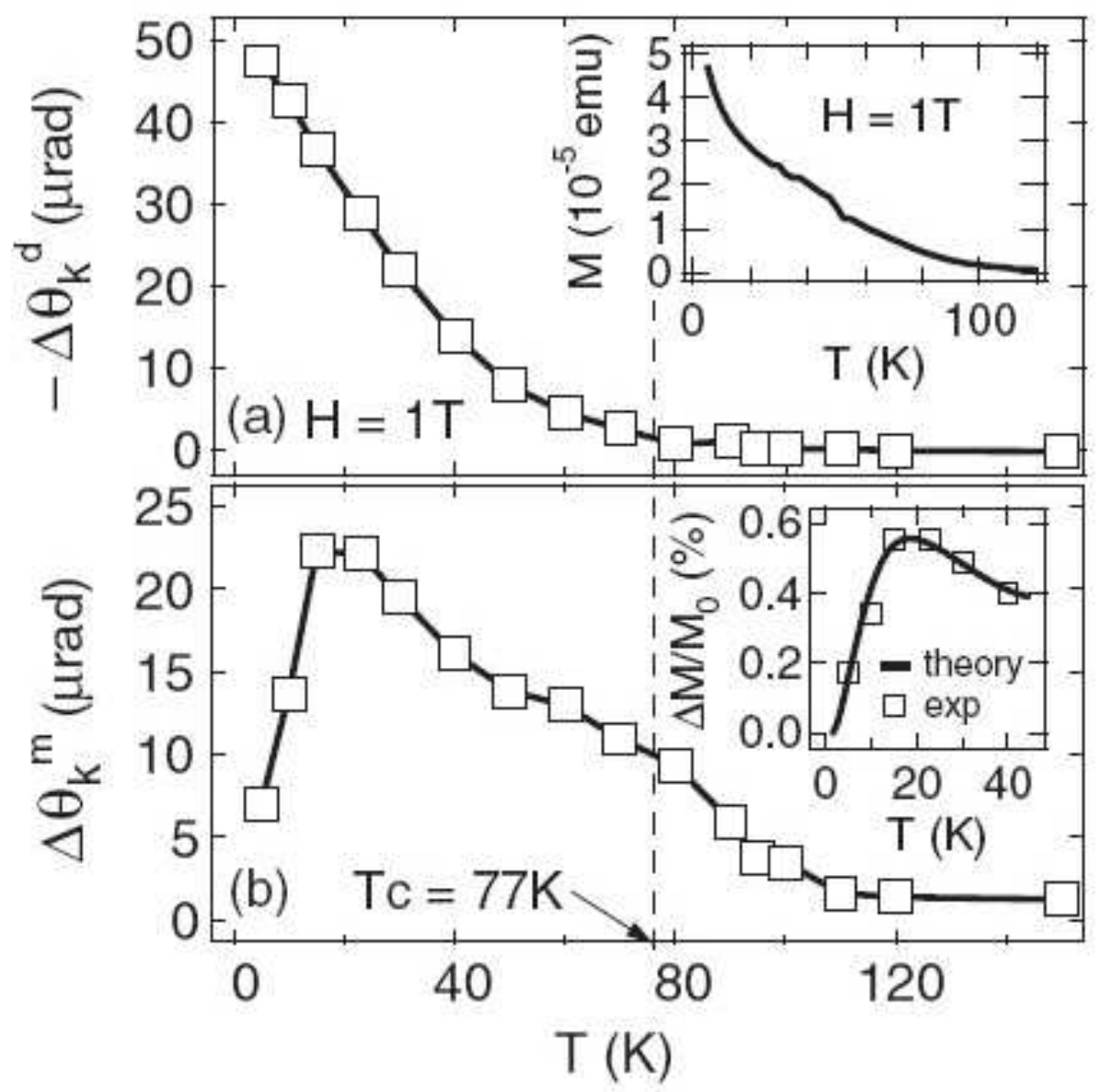

Figure 4.5: Decomposed demagnetization $-\Delta \theta_{K}^{d}(\mathrm{a})$ and enhanced magnetization $\Delta \theta_{K}^{m}(\mathrm{~b})$ components are plotted as a function of temperature. The static magnetization curve under a $1 \mathrm{~T}$ field is shown as the inset in (a). The simulation $\Delta M / M_{0}$ (thick line, $\Delta T_{C}=1.1 \mathrm{~K}$ and the hole/Mn ratio is 0.06) and experimental values (circles, normalized by static $\theta_{K}$ at $5 \mathrm{~K}$ ) of the photo-enhancement peak $\sim 20 \mathrm{~K}$ are shown as the inset of (b). 


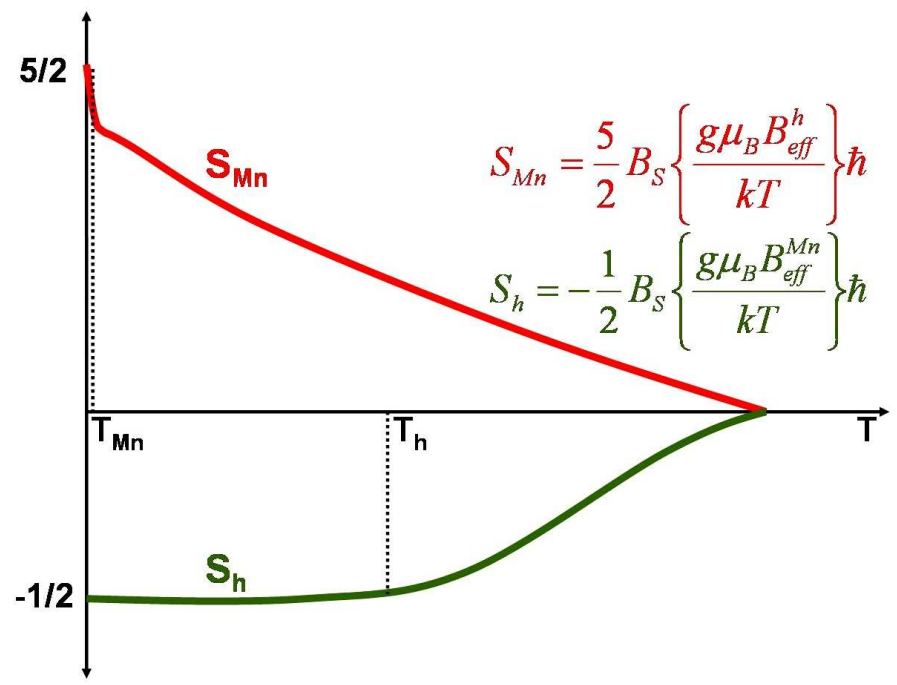

Figure 4.6: Qualitative temperature dependence of the Mn and hole spin polarization. The Mn spins are saturated only at $\mathrm{T}_{M n} \sim 0 \mathrm{~K}$, due to a small holes molecular field acting on them, $B_{\text {eff }}^{h} \ll B_{\text {eff }}^{M n}$. The holes, seeing a much larger Mn molecular field, are saturated bellow $\mathrm{T}_{h} \sim 20 \mathrm{~K}$.

at a temperature on the order of $\mathrm{T}_{h}$.

Next we present a simple theoretical calculation to simulate the Mn-hole correlationinduced peak around $20 \mathrm{~K}$. As we discussed, the hole-enhanced ferromagnetic correlation results in an increase of $\mathrm{T}_{C}\left(\Delta T_{C}>0\right)$. We calculate the experimental non-classical magnetization curve (inset of Fig. 4.5(b)) based on a modified Weiss mean-field model to take into account the $H_{p d}$ correlation [17]:

$\frac{M\left(T, \Delta T_{C}\right)}{M_{0}}=B_{S}\left[-3 \frac{T_{C}+\Delta T_{C}}{T} \gamma S^{\star} B_{s}\left(-3 \frac{T_{C}+\Delta T_{C}}{T} \gamma^{-1} S^{\star} \frac{M\left(T, \Delta T_{C}\right)}{M_{0}}+\frac{g_{h} \mu_{B} H}{k_{B} T}\right)-\frac{g_{i} \mu_{B} H}{k_{B} T}\right]$

where $B_{S, s}(x)$ is the Brillouin function, $\gamma$ is the square root of the hole and Mn density ratio $\sqrt{p / n_{i}}, g_{i}\left(g_{h}\right)$ is the Mn (hole) $g$ factor, and $S^{\star}=\sqrt{s S /(s+1)(S+1)}$. The Mn and hole spins, $\mathrm{S}$ and $\mathrm{s}$, are $5 / 2$ and $1 / 2$, respectively. At each given $\Delta T_{C}$, the magnetization 
enhancement $\Delta M / M_{0}$ is solved self-consistently as a function of lattice temperature. The calculated temperature-dependent $\Delta M / M_{0}$ and experimental $\Delta \theta_{K}^{m}$ (normalized by static $\theta_{K}$ at $5 \mathrm{~K}$ ) up to $40 \mathrm{~K}$ are shown in the inset of figure $4.5(\mathrm{~b})$ for $\Delta T_{C}=1.1 \mathrm{~K}$. The results of the calculation compare well with the experimental photo-enhancement peak. In addition, we can also estimate $\Delta T_{C}$ using an analytical expression derived from the Zener model, $\Delta T_{C}=\frac{1}{3} T_{C} \times \Delta p / p[22]$. Knowing the ratio of photoexcited and background hole densities $\Delta p / p \sim 2 \%$, we estimate $\Delta T_{C} \sim 0.5 \mathrm{~K}$, in reasonable agreement with the value used in the simulation. The photoexcited transient electrons have little effect on $\Delta \theta_{K}$, because of the short free electron lifetime $(<1 \mathrm{ps})$ [95]. In addition, the standard thermal demagnetization seen at temperatures below $15 \mathrm{~K}$ (Fig. 4.4(a)) via heat transfer from phonons, is similar to the slow demagnetization component reported in [97, 44].

\subsubsection{Signatures of phase transition}

Thus far our discussion has been based on a situation involving a fixed magnetic field of $1 \mathrm{~T}$, which is important at low temperatures in order to eliminate other distractions (such as magnetization reorientation effects). It is worthwhile, however, to consider the field dependence of the magnetization enhancement at temperatures just above $\mathrm{T}_{C}$ and possible transient signatures of photoinduced critical phenomena, e.g., a paramagnetic to ferromagnetic phase transition via the pump-induced increase of $\mathrm{T}_{C}$. Figure 4.7 presents the field dependence of the magnetization enhancement $\Delta \theta_{K}^{m}$ and demagnetization $-\Delta \theta_{K}^{d}$ at $78 \mathrm{~K}$. Here the most interesting aspects lie in the fact that $\Delta \theta_{K}^{m}$ is relatively constant across a wide field range $(0.2-1 \mathrm{~T})$ and only drops off close to $0 \mathrm{~T}$. It is critical to note that in the absence of a magnetic field, even though spontaneous symmetry breaking (i.e., ferromagnetic 
correlation) is present due to $\Delta \mathrm{T}_{C}>0$, the transient pump-induced magnetization above $\mathrm{T}_{C}$ always reduces to zero at small external field, because of the lack of ordering between individual magnetic domains. A below-threshold $\mathrm{H}$ field is not able to activate subsequent growth of magnetic domains, even though they are nucleated via photoinduced long-range ferromagnetic correlation. Therefore, the $1.1 \mathrm{~K}$ increase of $\mathrm{T}_{C}$ and the substantial transient magnetization enhancement at a small field of $0.05 \mathrm{~T}$ suggest a transient photoinduced paramagnetic to ferromagnetic phase transition. Finally, the decrease (increase) of $\Delta \theta_{K}^{m}$ $\left(-\Delta \theta_{K}^{d}\right)$ observed from 2 to $7 \mathrm{~T}$ is expected from the larger static magnetization achieved at higher fields, resulting in the smaller photoinduced changes, as indeed shown by the data.

Next, we briefly discuss the observed 100 ps time scale delay for the establishment of the FM phase above $\mathrm{T}_{C}$. The photo-induced magnetization changes at $78 \mathrm{~K}\left(>T_{C}\right)$, are plotted as function of time at different magnetic fields in figure 4.8(a). Very importantly, the build-up time $\tau$ is found to strongly depend on the applied magnetic field (Fig. 4.8(b)). Two distinct regimes are identified for FM phase formation: a formation time decreasing with increasing field up to $0.8 \mathrm{~T}$, and a rather constant, fast formation time at higher magnetic fields up to $6.5 \mathrm{~T}$. In the first regime, the experimental data (Fig. 4.8(b) inset) indicates that the build-up time of the FM correlation is roughly on the same order as the calculated inverse magnon frequency - the collective response time of the many-body spin system. This is consistent with the implication that the build-up of spin correlations and the formation of the FM phase take a least one oscillation cycle of the collective response in the non-equilibrium many body spin system [36]. On the other hand, the system exhibits a much faster and relatively constant formation time of the FM phase at high magnetic 


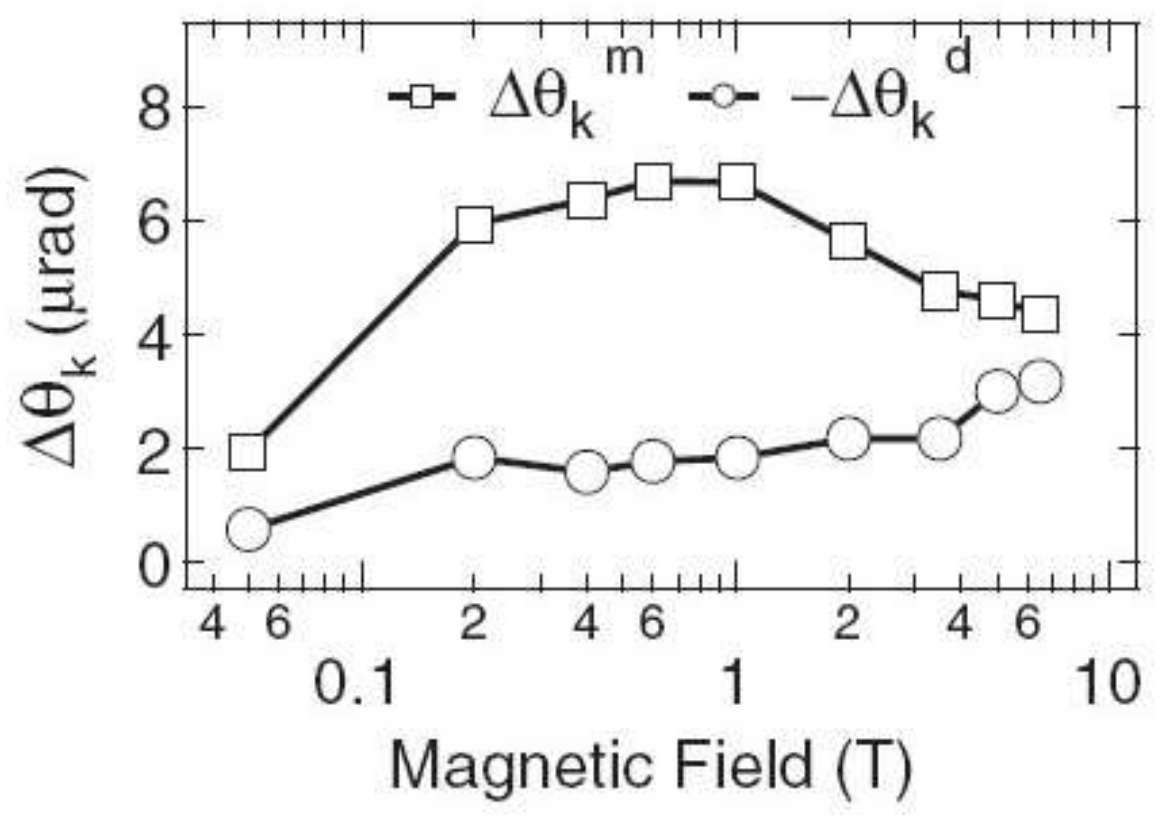

Figure 4.7: Magnetic field dependence of photoinduced magnetization enhancement $\Delta \theta_{K}^{m}$ and demagnetization $-\Delta \theta_{K}^{d}$ components $1 \mathrm{~K}$ above $\mathrm{T}_{C}$, at $78 \mathrm{~K}$.
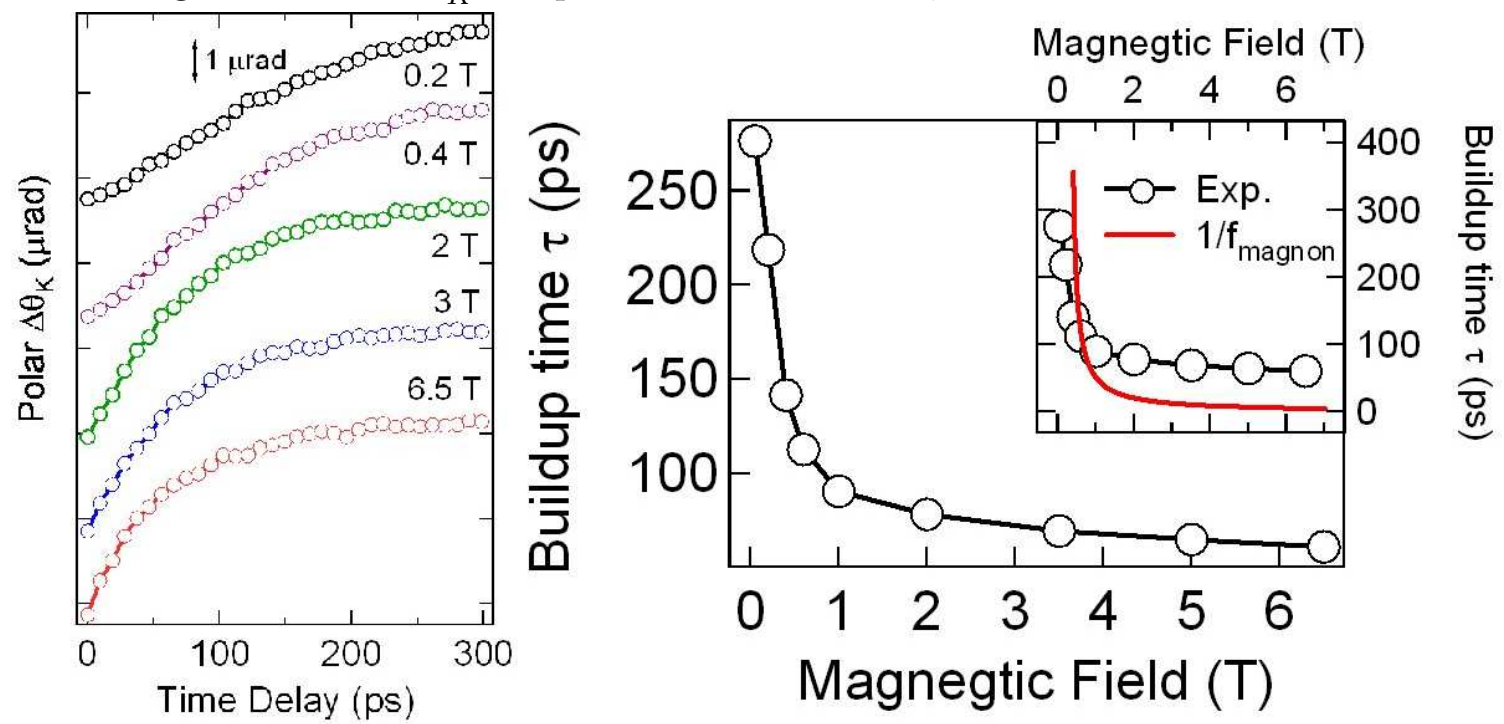

Figure 4.8: Magnetic field dependence of the establishment for FM phase above critical temperature $\mathrm{T}_{C}(77 \mathrm{~K})$. (left) The photo-induced magnetization at $78 \mathrm{~K}$ versus time at different magnetic fields. (right) The build-up time $\tau$ for FM phase versus magnetic field up to $6.5 \mathrm{~T}$. Inset: calculated inverse magnon frequency as a function of magnetic field is plotted together with experimental build-up times for FM phase. 
fields, when the collective excitations evolve on significantly shorter time scales. This observation may point to extrinsic factors affecting the micro-magnetic processes, expected from inhomogeneous magnetic domain nucleation due to disorder, and continuing growth of magnetic domains, which become dominant at high field.

Figure 4.9 shows the transient signature of ultrafast photo-creation of ferromagnetic order above $\mathrm{T}_{C}$. We plot the transient magnetization dynamics, emphasizing magnetization enhancement, under $0 \mathrm{~T}$ and $0.3 \mathrm{~T}$ magnetic fields, at three different characteristic temperatures: below $\mathrm{T}_{C}(70 \mathrm{~K})$, slightly above $\mathrm{T}_{C}(78 \mathrm{~K})$, and above $\mathrm{T}_{C}(85 \mathrm{~K})$. Most intriguingly, the photoinduced magnetization enhancement at $78 \mathrm{~K}$ is almost identical to the below $-\mathrm{T}_{C}$ response at $70 \mathrm{~K}$ under a small magnetic field - both in terms of amplitude and of magnetization build-up time (Fig. 4.9 (d) and (e)). In the absence of the magnetic

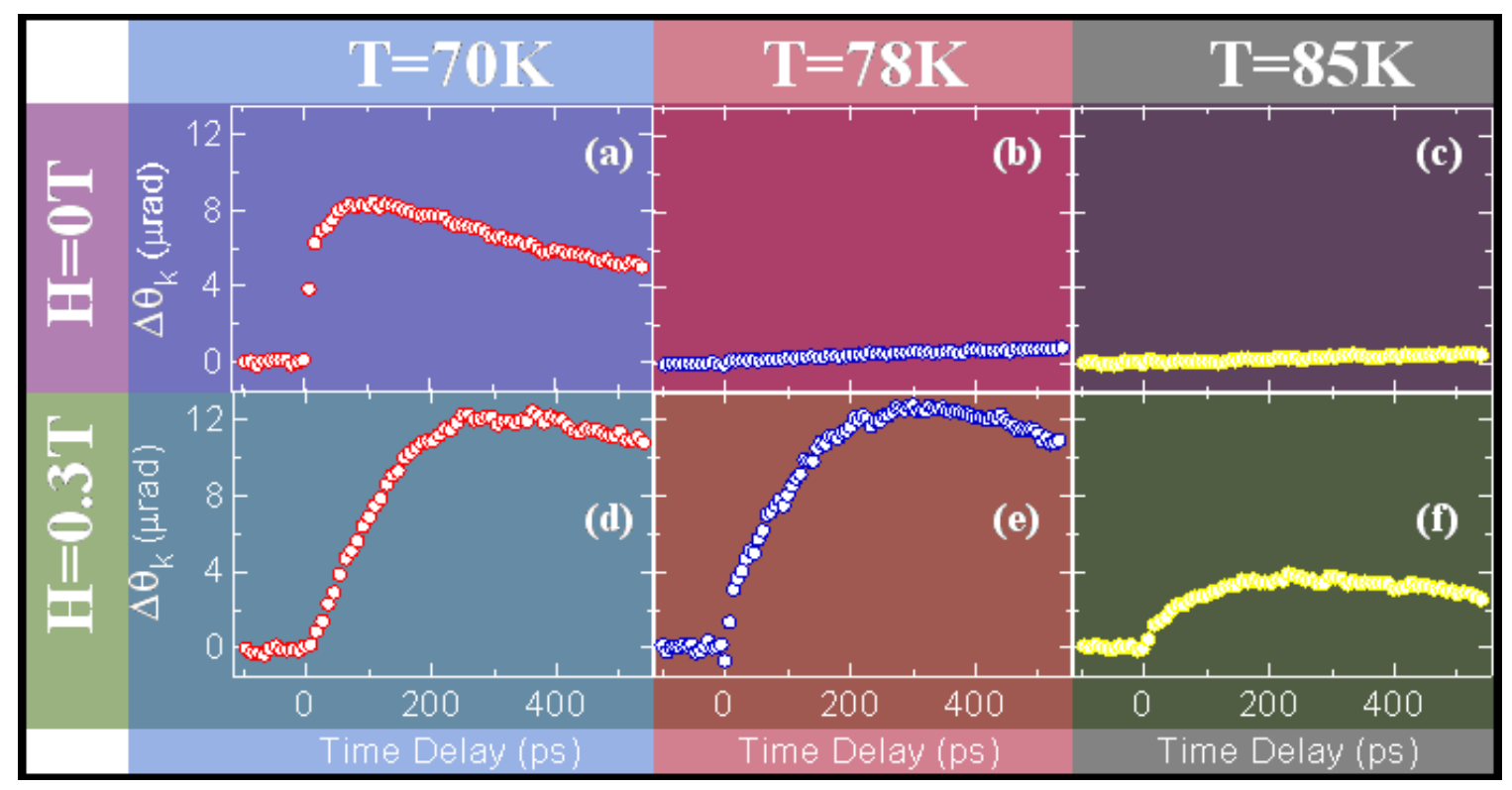

Figure 4.9: Transient magnetization enhancement at $70 \mathrm{~K}, 78 \mathrm{~K}$, and $85 \mathrm{~K}$, under $0 \mathrm{~T}$ (a-c) and $0.3 \mathrm{~T}(\mathrm{~d}-\mathrm{f})$ external magnetic field. The response at $78 \mathrm{~K}$ resembles the below- $\mathrm{T}_{C}$ response at $70 \mathrm{~K}$ under a small magnetic field, both in terms of amplitude and build-up time. 
field, the $78 \mathrm{~K}$ response is negligible, and similar to the above- $\mathrm{T}_{C}, 85 \mathrm{~K}$ response (Fig. 4.9 (b) and (c)). These results clearly reveal that the photo-excited magnetic state at $78 \mathrm{~K}$ is similar to the $70 \mathrm{~K}$ ferromagnetic state, even though the unexcited, ground state at $78 \mathrm{~K}$ is paramagnetic, just like the state at $85 \mathrm{~K}$. The data also corroborates our interpretation that in order to detect macroscopic magnetization changes, an above-threshold, external magnetic field is needed to activate the growth of magnetic domains. The single phase in these domains is nucleated via long range correlation enabled by photoexcited holes.

\subsubsection{Studies on quasi-2D MBE-grown GaMnAs}

Since the results on our thin film MBE grown sample were very intriguing, we expanded our experiments to study enhancement of FM on a quasi-2D layer of GaMnAs (sample B). Since the interaction between substitutional Mn atoms and free holes generates

FM in GaMnAs, it is necessary to increase both Mn and free hole concentrations to achieve high $\mathrm{T}_{C}$. However, the hole concentration in heavily Mn-doped GaMnAs is much lower than the Mn concentration, due to the formation of interstitial Mn atoms which act as double donors in GaAs. At the same time, interstitial Mn and substitutional Mn easily form complex antiferromagnetic complexes. In an effort to increase $\mathrm{T}_{C}$, by decreasing the interstitial Mn concentration and increasing the hole concentration, $\delta$-doped (Ga,Mn)As has been grown [58]. These samples have a maximum $\mathrm{T}_{C}$ of $172 \mathrm{~K}$, achieved with optimum overlap of a modest Mn concentration and the wavefunction of 2-dimensional hole gas (2DHG) provided by a remote layer of Be-doped AlGaAs (Fig. 4.10).

We expect that in the quasi-2D sample the enhancement of FM is much stronger than in the previous thicker layers, due to confinement effects and optimum overlap of Mn 


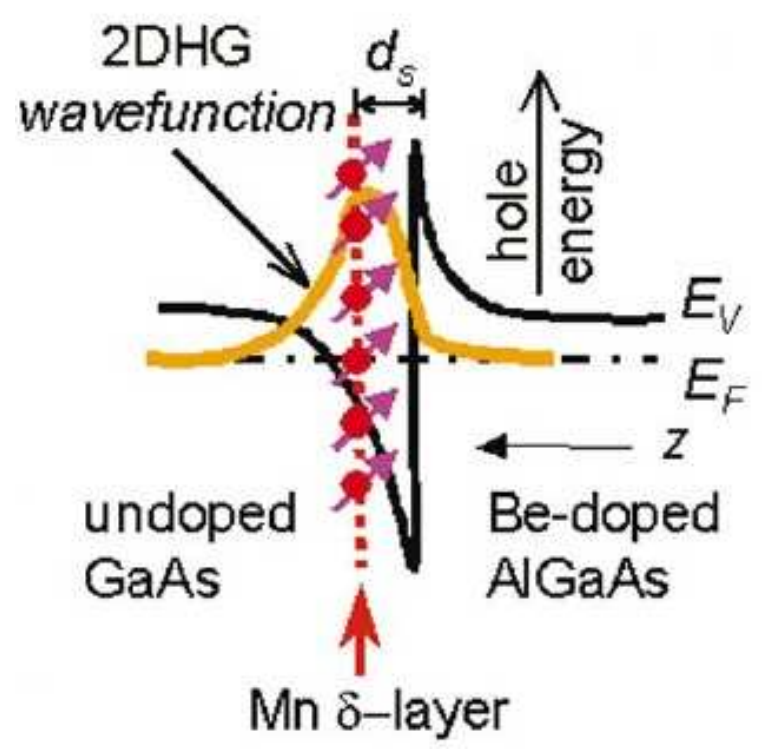

Figure 4.10: From ref. [58]: Schematic diagram of the valence band profile of the Mn $\delta-$ doped GaAs/Be-doped p-type AlGaAs heterostructure, the 2DHG wavefunction, and Mn dopants. $\mathrm{E}_{V}$ and $\mathrm{E}_{F}$ are the valence band top and the Fermi energy, respectively. $\mathrm{z}$ is the growth direction.

concentration and hole wavefunction. Sample B consists of $10 \mathrm{~nm}$ of $\mathrm{Ga}_{0.92} \mathrm{Mn}_{0.08} \mathrm{As}$ grown by LT-MBE on a GaAs buffer layer on top of GaAs. The magnetization easy axes are in plane, [100] up to $10 \mathrm{~K}$, and [100]at higher temperatures up to $\mathrm{T}_{C}$ of $140 \mathrm{~K}$ (Fig. 4.11).

As with sample A, we start our study with room temperature differential reflectivity measurements, shown in figures 4.12 and 4.13. The results are somewhat different than sample A's. Although the penetration depth of $400 \mathrm{~nm}$ radiation is only about $14 \mathrm{~nm}$, in this sample it is enough to reach the substrate and photo-excite carriers in it. Therefore, photo-excited charges are likely transferred from the underlying GaAs layer into the magnetic layer. Thus, we expect a completely different magnetization dynamics in sample B than in sample A, which is indeed, what we observe.

First, unlike in sample A, the MOKE and MCD signals do not coincide (Fig. 


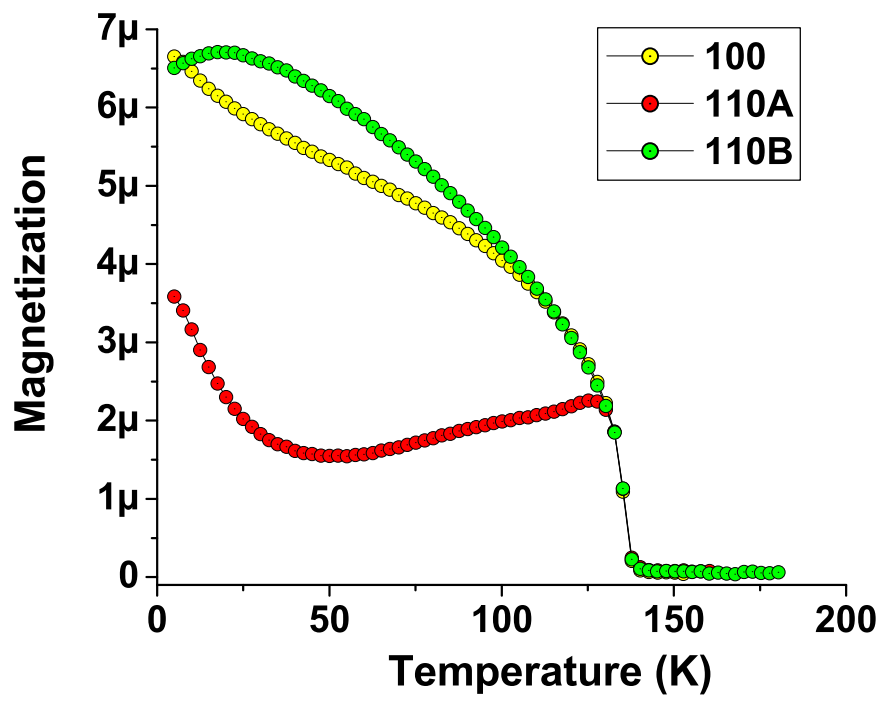

Figure 4.11: Sample B magnetization versus temperature along two in-plane easy axes shows a switch from [100] to [110] around $10 \mathrm{~K}$

4.14). This means that the real and imaginary part of the off diagonal susceptibility tensor component, $\theta_{K}$ and $\eta_{K}$, have different, time-varying pre-factors in their proportionality to M (Eqns. 2.16-2.17). These pre-factors depend on the charge dynamics in the sample, and are heavily influenced by the charge transfer from GaAs. As a consequence, neither MOKE nor MCD signals are purely magnetic, but are also influenced by the charge dynamics. Therefore, we choose to conduct our studies by monitoring both MOKE, $\theta_{K}$, and $\Delta R / R$ dynamics.

We saturate the magnetization perpendicular to the sample with an external magnetic field of $1 \mathrm{~T}$, and measure its dynamics reflected in the magneto-optical signals versus temperature. The results are presented in figure 4.15. Strikingly, there is an abrupt change around $72 \mathrm{~K}$ in the MOKE signal. We verify that it does not depend on the applied magnetic field. Since the MOKE signal does not represent only magnetization dynamics, but 


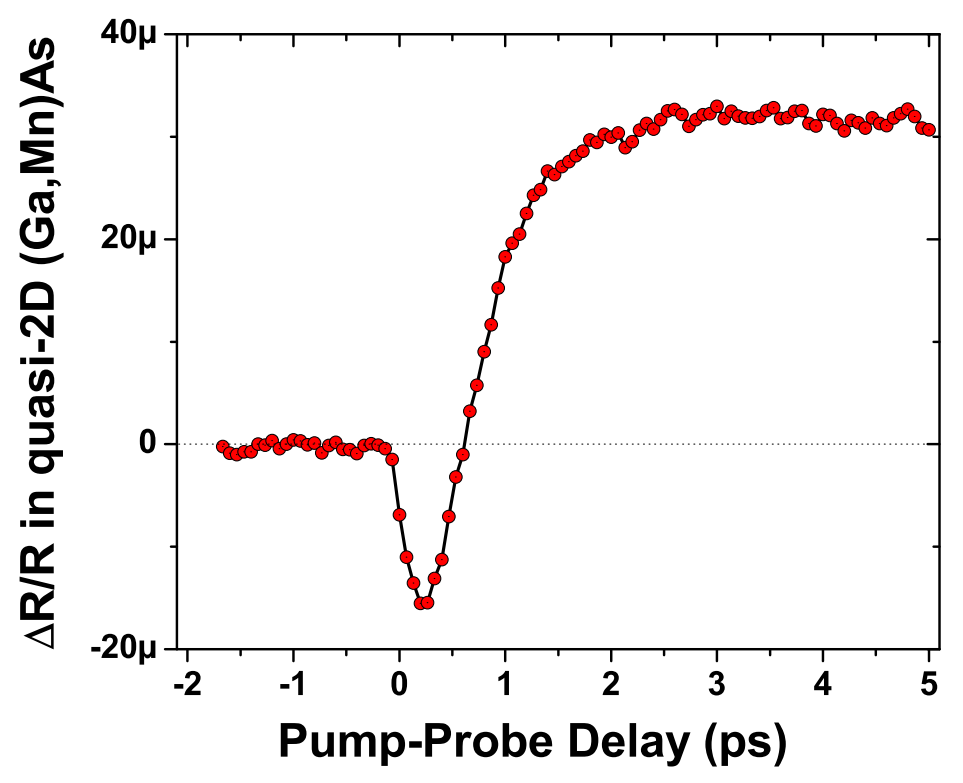

Figure 4.12: Room temperature differential reflectivity measurements in quasi-2D (Ga,Mn)As, (i.e., sample B) at the ps timescale. $\Delta \mathrm{R} / \mathrm{R}$ is initially negative, due to initial absorption, followed by a rapid reflectivity rise.

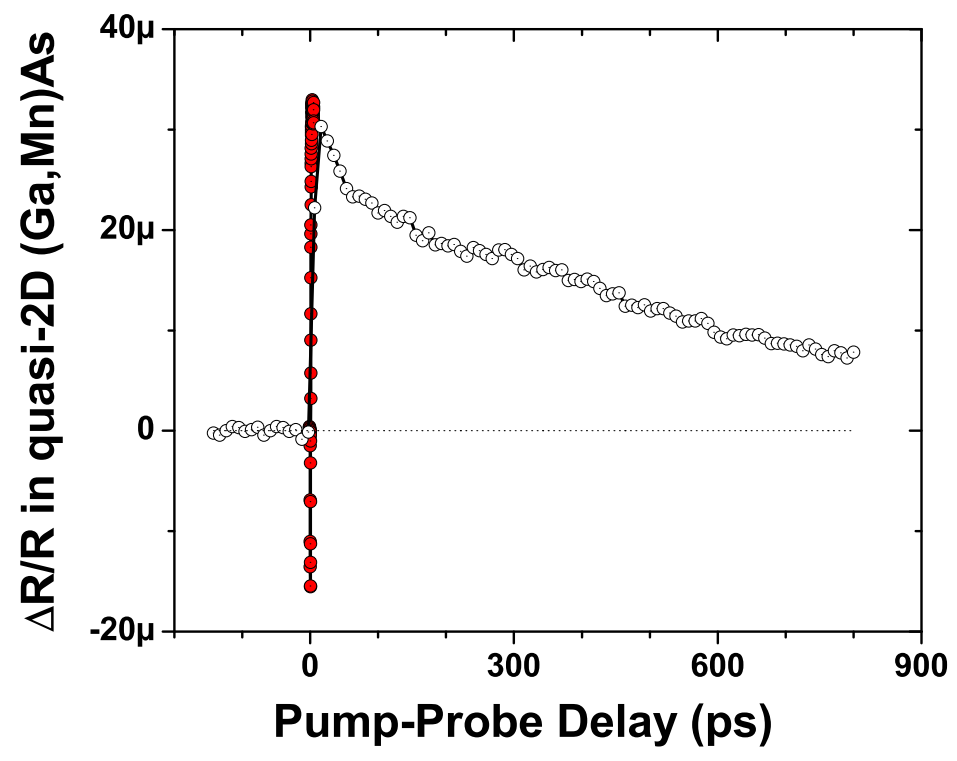

Figure 4.13: Room-temperature $\Delta \mathrm{R} / \mathrm{R}(\mathrm{t})$ at the ns timescale. Following the rapid dip and recovery of the differential reflectivity signal, $\Delta \mathrm{R} / \mathrm{R}(\mathrm{t})$ begins its final decay process towards zero at the ns timescales. 


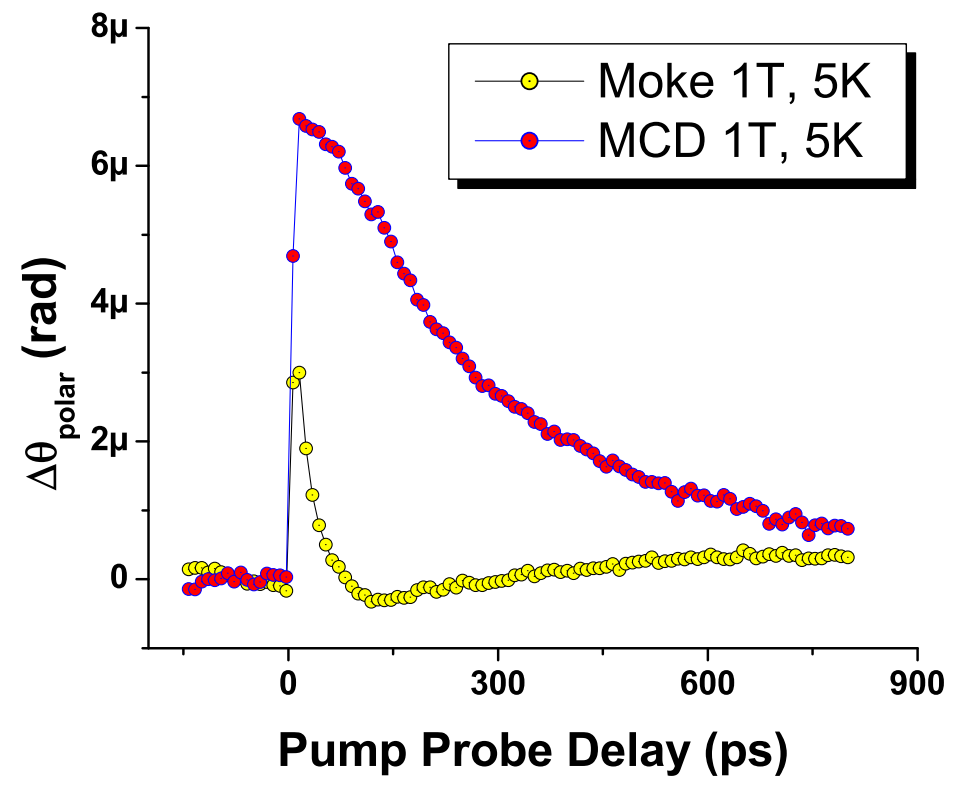

Figure 4.14: A comparison of MOKE and MCD signals in quasi-2D (Ga,Mn)As shows considerable differences due to charge transfer from underlying layer of GaAs.

charge dynamics as well, we also study the temperature dependence of the differential reflectivity dynamics, in figure 4.16. Surprisingly, a significant change in the dynamic $\Delta R / R$ signal is again observed around $\mathrm{T}=72 \mathrm{~K}$. This clearly indicates that there is a very strong interplay between charge dynamics and spins in the system, hidden in the static measurements. Since GaAs alone does not show any signs of a sudden transition in the differential reflectivity measurement around $72 \mathrm{~K}$, we conclude that there is a subtle, sudden change in the magnetic properties of this quasi-2D ( $\mathrm{Ga}, \mathrm{Mn})$ As system. One possibility for this is the transition from a half-filled to beyond half-filled valence band (VB). Before the transition, the photoexcited holes decay to the center of the Brillouin zone and find themselves spin-polarized, since the VB is less than half-full. After the transitions, the same holes decay to the top of the VB spin unpolarized. Hence, even though the overall static mag- 


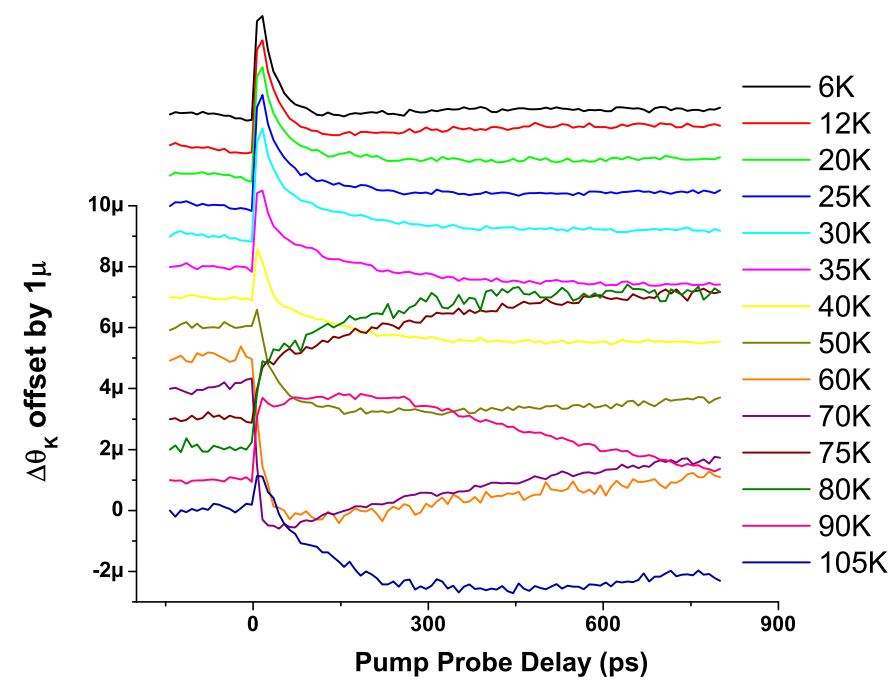

Figure 4.15: Dynamics of the MOKE signal $\Delta \theta_{K}$ for different temperatures show abrupt changes around $72 \mathrm{~K}$, absent in the static magnetization curve.

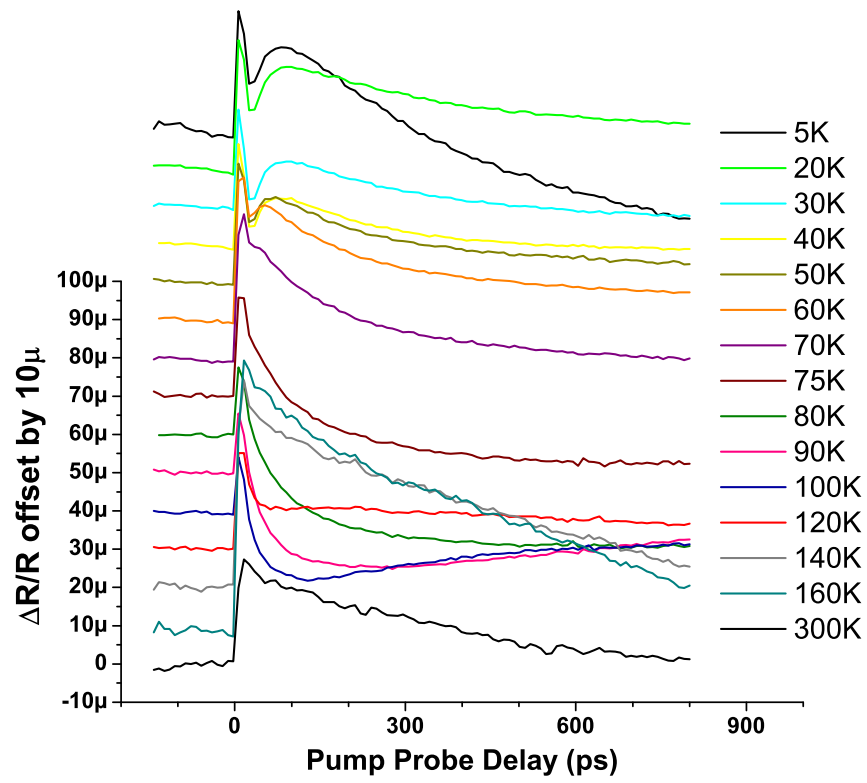

Figure 4.16: Dynamics of the differential reflectivity signal $\Delta R / R$ for different temperatures also show abrupt changes around $72 \mathrm{~K}$. 
netic properties do not change much (since the static holes polarization varies smoothly across the transition), the change in the photoexcited holes polarization would be enough to drastically change the magnetization dynamics and the transient differential reflectivity, sensitive to the distribution in the VB.

Comparing the MOKE and the $\Delta \mathrm{R} / \mathrm{R}$ signals at particular representative time delays ( $<1 \mathrm{ps}, 100 \mathrm{ps}$, and $0.5 \mathrm{~ns}$, respectively) with the static properties, certain trends become more clear (Fig. 4.17 and 4.18). For example, the signals are pretty much constant up to $30 \mathrm{~K}$, when they begin to gradually change (MOKE drops, $\Delta \mathrm{R} / \mathrm{R}$ increases). At around $72 \mathrm{~K}$, both MOKE and $\Delta \mathrm{R} / \mathrm{R}$ experience a sudden transition. Beyond this temperature, they experience an extreme (MOKE has a minimum, $\Delta \mathrm{R} / \mathrm{R}$ has a maximum) up to $\mathrm{T}_{C}$

We have uncovered some very interesting aspects of the charge-spin interplay in the quasi-2D (Ga,Mn)As sample, visible only in the dynamical magneto-optical properties of the system but not in static measurements. We observed an unexpected transition around $72 \mathrm{~K}$, which we tentatively attribute to a half-metal to metal transition. 


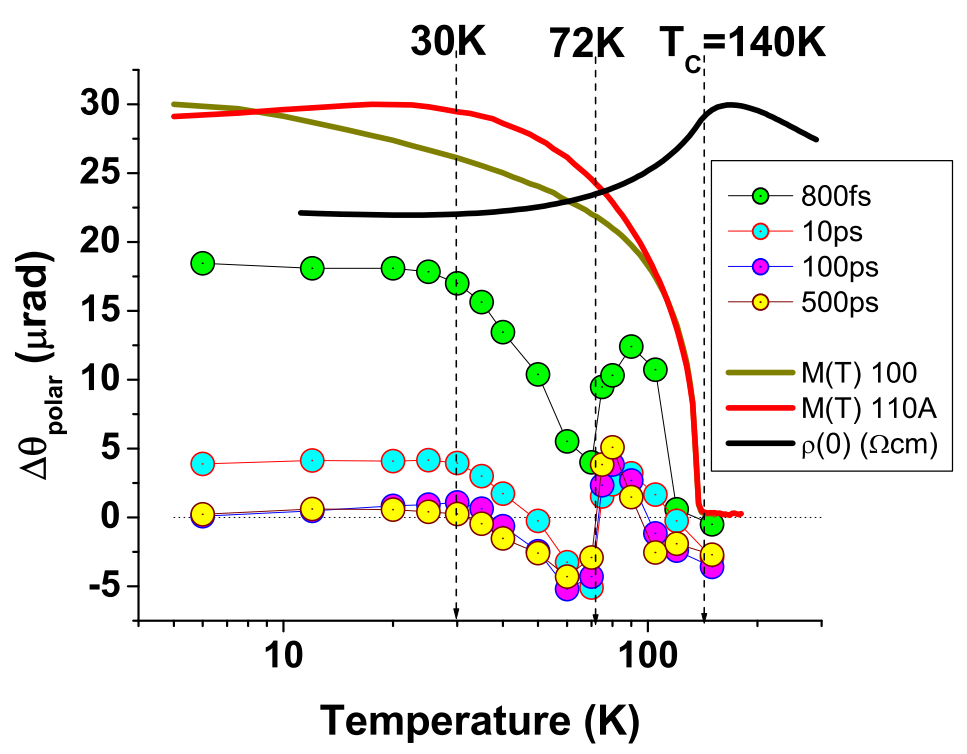

Figure 4.17: The temperature dependence of the MOKE signal at time delays $\tau<1 \mathrm{ps}, 100$ ps, and $0.5 \mathrm{~ns}$, shows an abrupt transition at $72 \mathrm{~K}$; the static properties $\mathrm{M}(\mathrm{T})$ and $\rho(\mathrm{T})$ do now show such changes.

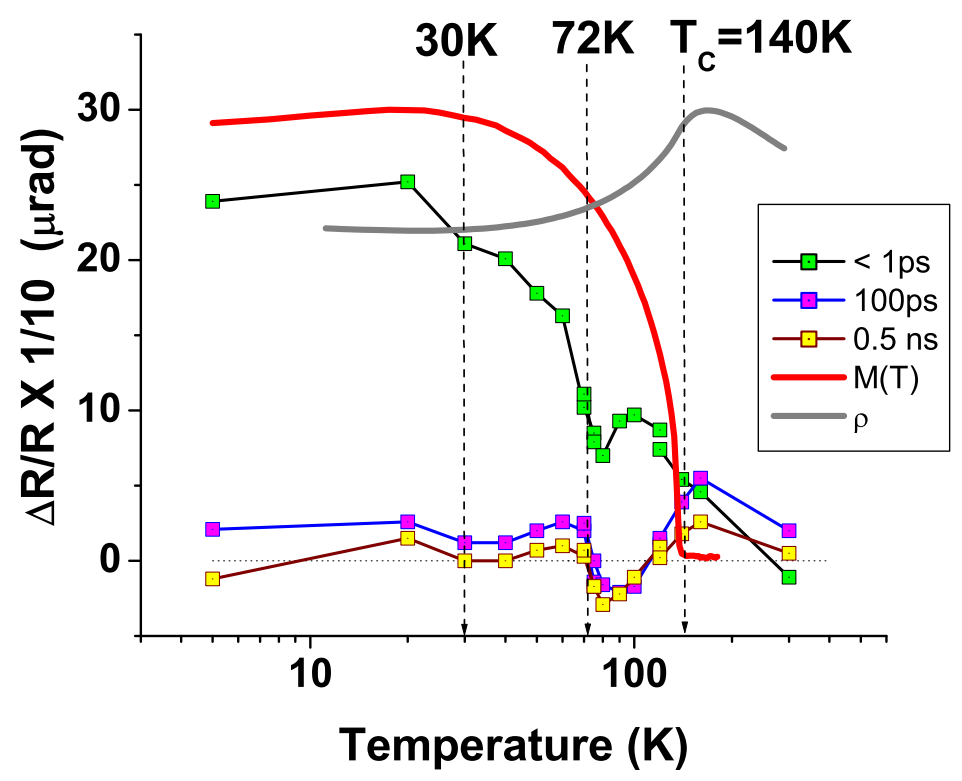

Figure 4.18: The temperature dependence of the $\Delta \mathrm{R} / \mathrm{R}$ signal at time delays $\tau<1 \mathrm{ps}, 100$ ps, and $0.5 \mathrm{~ns}$. It also shows a transition around $72 \mathrm{~K}$, unlike the static properties. 


\subsection{Magnetization dynamics in II-PLM (Ga,Mn)As}

The use of ion implantation and pulsed-laser melting (II-PLM) to incorporate dopants in semiconductors was introduced in the late 1970s and remains an ongoing topic of research today $[75,74,83]$. Compared to MBE, this approach has fewer restrictions on the choice of host semiconductor and alloying species, enabling thus the investigation of a wide range of systems. PLM has been used to synthesize both equilibrium phases and metastable phases, due to the $10^{9}-10^{10} \mathrm{~K} / \mathrm{s}$ quench rates achievable using nanosecond laser pulses. The incorporation of impurities in semiconductors at concentrations exceeding maximal solubility limits without precipitation or the formation of second phases is due to this fast quenching.

II-PLM is a simple and highly versatile method for the formation of ferromagnetic $\mathrm{Ga}_{1-x} \mathrm{Mn}_{x} \mathrm{As}$. The general features of temperature and field dependencies of magnetization, sheet resistivity, and Hall resistivity in II-PLM samples agree with results reported for annealed $\mathrm{Ga}_{1-x} \mathrm{Mn}_{x}$ As films synthesized using LT-MBE. Furthermore, there is good quantitative agreement found between results from II-PLM films characterized by their maximum substitutional Mn concentration $\mathrm{x}$, and from LT-MBE films of equivalent substitutional Mn concentration. This shows that the II-PLM films' magnetic properties are governed by the film region with the maximum $\mathrm{Mn}_{G a}$ concentration.

The rapid melting and recrystallization associated with PLM leads to a high level of $\mathrm{Mn}$ incorporation in the regrown layer, while suppressing the formation of second phases. During PLM, the ion-implanted region of the film melts, solidifies epitaxially, and cools to room temperature within a few hundred nanoseconds. As a result of this high-temperature 
processing $\left(\mathrm{T}_{\text {Melt }}=1511 \mathrm{~K}\right.$ for GaAs), films produced using II-PLM are free from interstitial Mn. Interstitial Mn act as donors in $\mathrm{Ga}_{1-x} \mathrm{Mn}_{x} \mathrm{As}$ and reduce the strength of the $\mathrm{FM}$ order by lowering the hole concentrations. By contrast, post-growth annealing is typically carried out in LT-MBE films to remove interstitial Mn and achieve high $\mathrm{T}_{C}$.

Besides being a much more versatile growth technique, with potential prospect of large scale application, II-PLM allows for doping of $\mathrm{Ga}_{1-x} \mathrm{Mn}_{x}$ As samples with various other co-dopants, without having a dedicated piece of equipment (such as an effusion oven in the MBE growth chamber). For example, elements such as $\mathrm{S}$, or $\mathrm{P}$, can be easily implanted to replace As in $\mathrm{Ga}_{1-x} \mathrm{Mn}_{x}$ As to control the sample compensation (and thus, hole concentration) or easy axis direction.

\subsubsection{Charge dynamics}

We expand our study of FM phase enhancement via photoexcited transient holes to an II-PLM (Ga,Mn)As sample (sample C). Room temperature differential reflectivity measurements in this sample, shown in figure 4.19, differ substantially from those in the MBE-grown sample A, indicating a considerable difference in photoexcited charge dynamics. We observe an initial sharp dip in $\Delta \mathrm{R} / \mathrm{R}$, followed by slow recovery of the signal on a $250 \mathrm{ps}$ timescale. The initial negative change results from free carrier absorption. The lack of a sub-ps decay of the initial negative signal, which in LT-MBE was attributed to the ultrafast trapping of electrons (by $\mathrm{As}_{G a}$ antisite defects) and holes (by Ga vacancies), supports the claim that II-PLM (Ga,Mn)As has far fewer $\mathrm{As}_{G a}$ antisite defects than LTMBE (Ga,Mn)As. The subsequent recovery of the signal represents the recombination of the photo-excited carriers. It must be noted that in II-PLM (Ga,Mn)As, the charge carrier 
dynamics is further complicated by the fact that the charge density is not uniform within the sample, nor does it follow the Mn concentration profile precisely. In fact, more carriers are created close to the surface, where the pre-existing charge density is quite low, than at $50 \mathrm{~nm}$ within the sample, where the charge density reaches a maximum. Hence, we expect larger effects in magnetization ordering to come from the first few $\mathrm{nm}$ at the sample surface than from within.

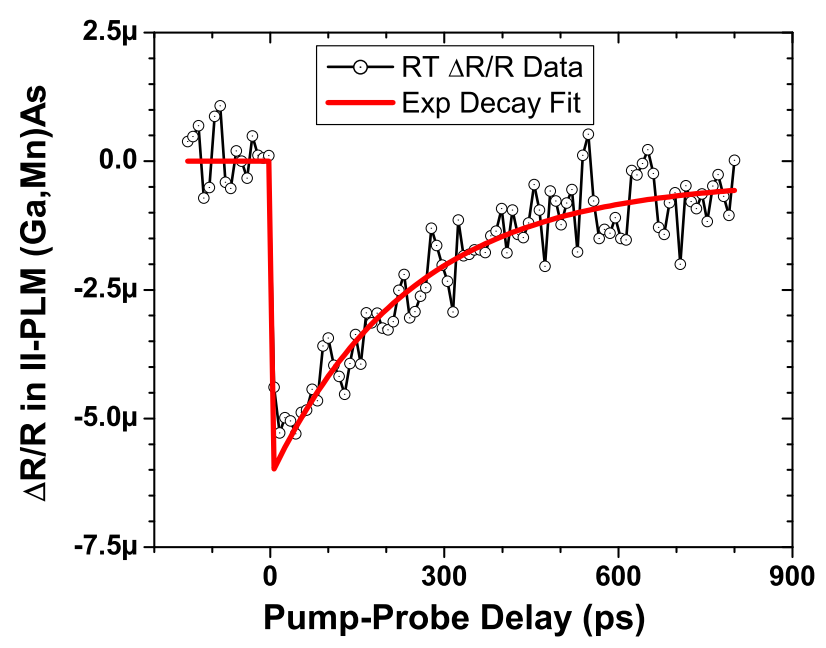

Figure 4.19: Room temperature differential reflectivity measurements in II-PLM (Ga,Mn)As, i.e., sample C. The transient pump-induced changes indicate ultrafast excitation of electron-hole pairs into high momentum states, followed by recombination with $\tau \sim 242$ ps.

\subsubsection{Giant ferromagnetic phase enhancement}

We first verify the overlap of the MOKE and MCD signals, on both the subps and ns timescales, to ensure the validity of our data interpretation. Shown in figure 4.20 are UV pump/NIR probe, polar MOKE spectroscopy measurements of magnetization amplitude temporal scans at $12 \mathrm{~K}$ and $20 \mathrm{~K}$. The photoinduced magnetization changes 
$\Delta \theta_{K}$ (normalized by total magnetization) are measured under $1 \mathrm{~T}$ magnetic field along the $\hat{z}$ direction, perpendicular to the sample surface. Again, the external magnetic field ensures that the magnetization is aligned along it and that by Kerr rotation angle one measures dynamics of the magnetization amplitude, not reorientation of the magnetization vector. Two mutually competing dynamic magnetization processes are observed, with very different temperature dependences. After the laser excitation, a negative $\Delta \theta_{K}$ is observed, indicating initial fast photoinduced demagnetization. On the hundreds of ps timescale, a distinct transient enhancement of magnetization, signaled by the positive $\Delta \theta_{K}$, follows. The dynamic enhancement of magnetic ordering is well explained by a transient strengthening of

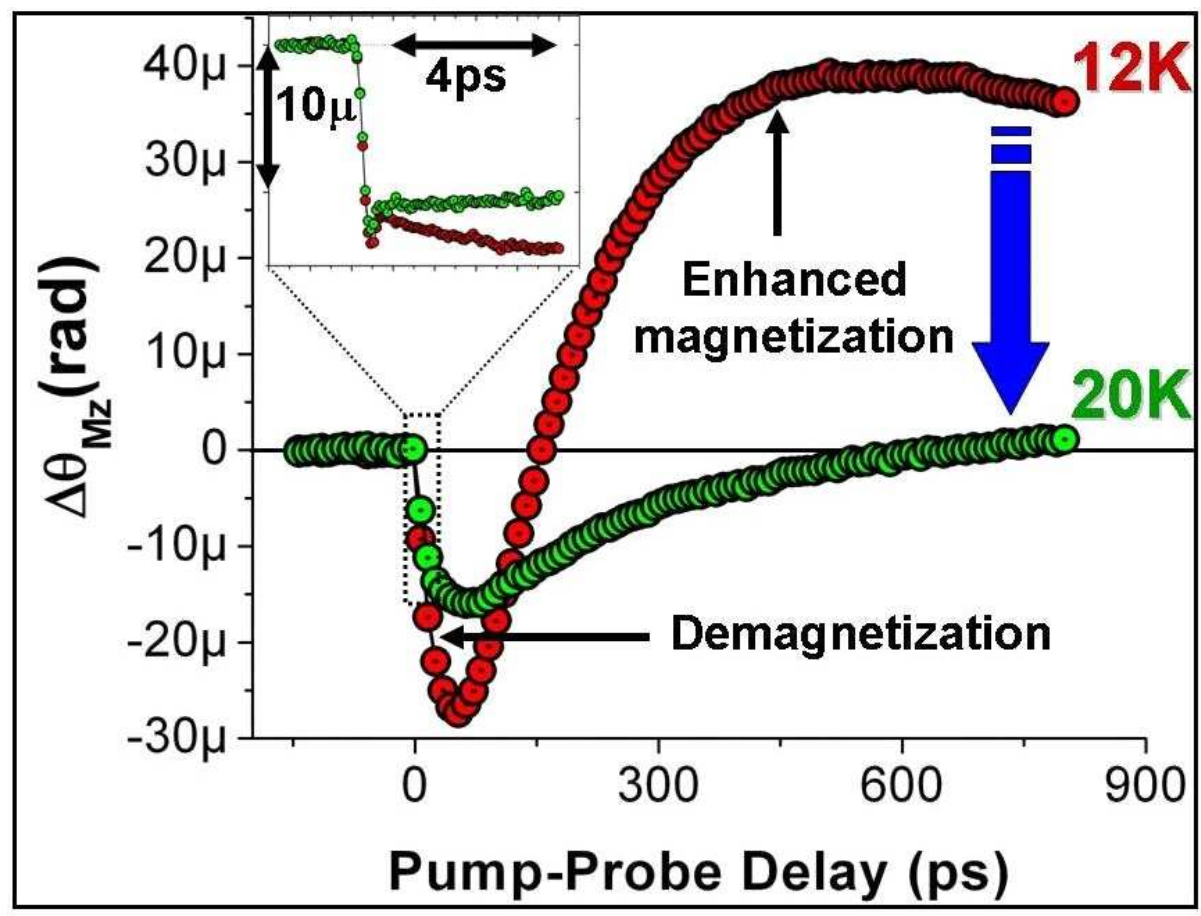

Figure 4.20: The dynamic traces of Kerr rotation angle, measuring magnetization amplitude, are fitted for demagnetization and enhanced magnetization components. The enhanced magnetization, visible on the hundreds of ps timescale of the $12 \mathrm{~K}$ trace (red), quickly disappears below $20 \mathrm{~K}$ (green). In contrast, the demagnetization (see inset) follows the static magnetization curve, and only disappears around $\mathrm{T}_{C} \sim 90 \mathrm{~K}$. 
the hole-mediated Mn-Mn interaction via the population of photoexcited holes, as previously observed in the LT-MBE sample, but there are subtle differences. Surprisingly, we find that the temperature dependence of the dynamic magnetization enhancement in this sample exhibits unexpected abrupt quenching at low temperatures, which is a strikingly different behavior than that observed in sample A.

Fitting the temporal scans taken at different lattice temperatures (Fig. 4.22) with these two components $\left(\Delta \theta_{K}^{d}\right.$ and $\left.\Delta \theta_{K}^{m}\right)$, and allowing each of the components a build-up $\left(\tau_{d}\right.$ and $\left.\tau_{m}\right)$ and a decay/ recovery time $\left(\tau_{d r}\right.$ and $\left.\tau_{c}\right)$, one can extract the magnitude of the demagnetization and magnetization enhancement components, plotted in figure 4.22:

$$
\Delta \theta_{K}=-\Delta \theta_{K}^{d}\left[1-\exp \left(-t / \tau_{d}\right)\right] \exp \left(-t / \tau_{d r}\right)+\Delta \theta_{K}^{m}\left[1-\exp \left(-t / \tau_{m}\right)\right] \exp \left(-t / \tau_{c}\right)
$$

Though the magnetization enhancement component is very large in magnitude at low temperatures $(\sim 10 \%$ of static magnetization at $6 \mathrm{~K})$, it shows unexpected quenching with increasing temperature, and disappears completely above $20 \mathrm{~K}$. In stark contrast to this behavior, the demagnetization component does not experience the same changes (inset of Fig. 4.20 ), but follows the static magnetization curve and disappears only around $\mathrm{T}_{C}=90 \mathrm{~K}$.

The hole-mediated exchange interaction between $\mathrm{Mn}$ ions is at the core of the observed ultrafast magnetization enhancement. The mechanism responsible for it is the photo-excitation of transient holes into high momentum states, from which they decay to the top of the valence bands, and strengthen the hole-mediated Mn-Mn exchange interaction on timescales of hundreds of ps. This is explained, as in the case of sample A, by a transient increase of the Curie temperature. However, the rapid termination of this process below $20 \mathrm{~K}$ is a completely new and puzzling phenomenon. 


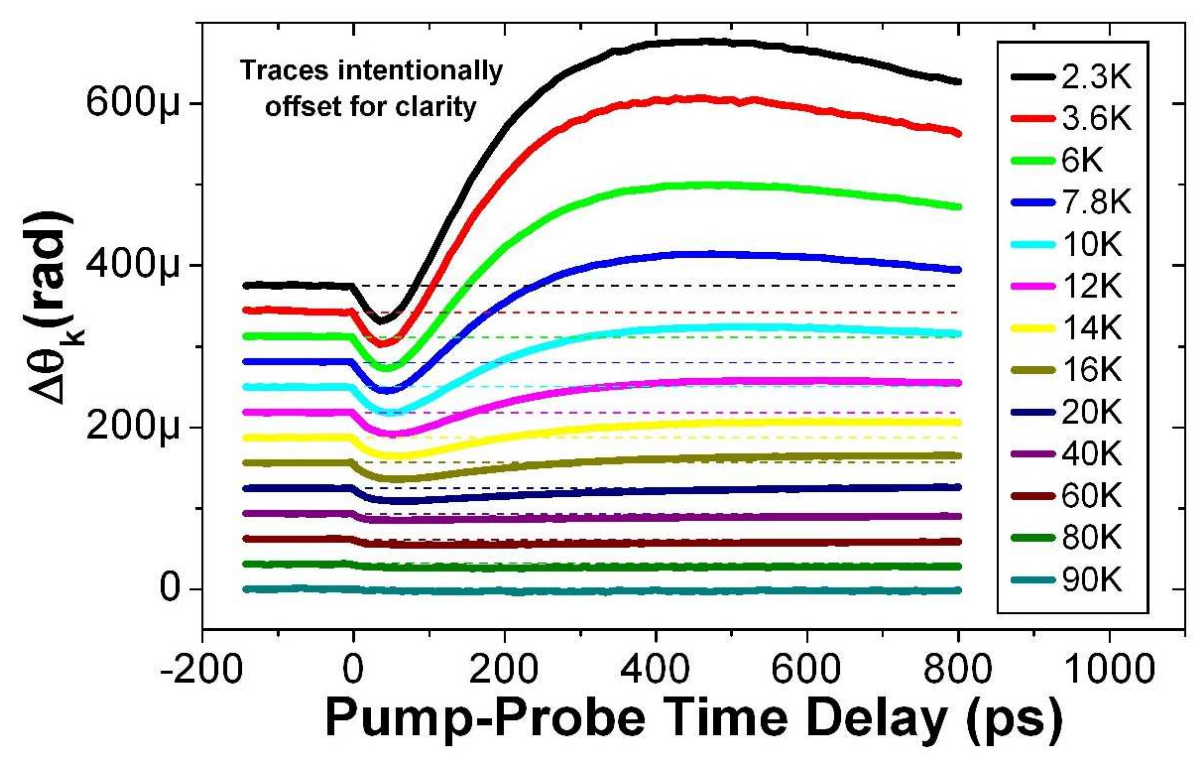

Figure 4.21: Photo-induced magnetization amplitude dynamics versus temperature up to $\mathrm{T}_{C}$ of $90 \mathrm{~K} . \Delta \theta_{K}^{m}$ disappears around $\mathrm{T}=20 \mathrm{~K}$, while $\Delta \theta_{K}^{d}$ persists all the way up to $\mathrm{T}_{C}$.

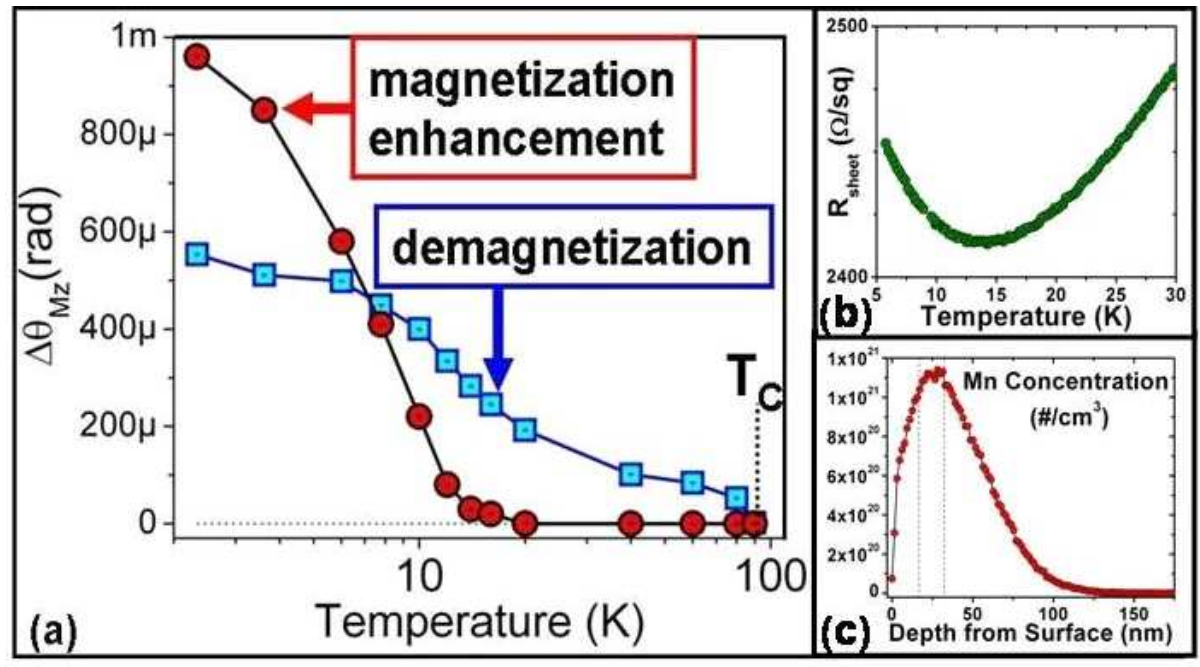

Figure 4.22: (a) Magnitude of magnetization enhancement (red circles) shows unexpected quenching below $20 \mathrm{~K}$, as opposed to demagnetization (X10, blue squares), which disappears around $\mathrm{T}_{C}$ of $90 \mathrm{~K}$. (b) Resistivity measurements show an insulator to metal transition below 20 K. (c) Non-uniform Mn doping profile. The densest Mn ions in the $\sim 10 \mathrm{~nm}$ layer about $30 \mathrm{~nm}$ deep into the sample are responsible for most of the magnetic signal observed. 


\subsubsection{Discussion}

One plausible explanation for the observed giant photo-enhanced FM phase is a photo-induced paramagnetic-to-FM phase transition in the top surface layers of the sample. Qualitatively, we explain this scenario as follows. Due to the non-uniform doping (Fig. 4.22(c)), some of the top layers are in a paramagnetic (PM) phase even at low temperatures, below $20 \mathrm{~K}$. These are the layers with low Mn and hole concentrations, i.e., close to the surface or deep into the sample, past the Mn peak. The pump pulse is absorbed by the sample following eqn. 3.8 , creating an exponentially decreasing population of hot holes. These extra photo-excited holes can induce a PM to FM phase transition. We must take notice that in this scenario, the photoexcited holes would be able to induce a phase transition only in those layers already close to the critical temperature point. Practically, the photoexcited holes do not penetrate deep enough to induce phase transitions in the deeper layers. Thus, as the temperature increases, the transition layers will be further from the surface, with a higher local $\mathrm{T}_{C}$, and higher $\mathrm{Mn}$ and hole density, as illustrated in figure 4.23. However, the relative density of photoexcited holes to background holes decreases further from the surface, and thus the holes have a smaller effect, and less strength in inducing phase transitions. Therefore, the size of the $\mathrm{PM} \rightarrow \mathrm{FM}$ transition layer decreases substantially as the temperature increases. As a consequence, $\Delta \mathrm{M}$ is quickly quenched. At any given temperature, at a specific depth, the material is FM before excitation. In this

case, the photoexcited holes only strengthen the FM order, inducing a smaller increase in $\Delta \mathrm{M} / \mathrm{M}$ comparable to the values observed in LT-MBE (Ga,Mn)As (i.e., maximum $0.5 \%$ ). 


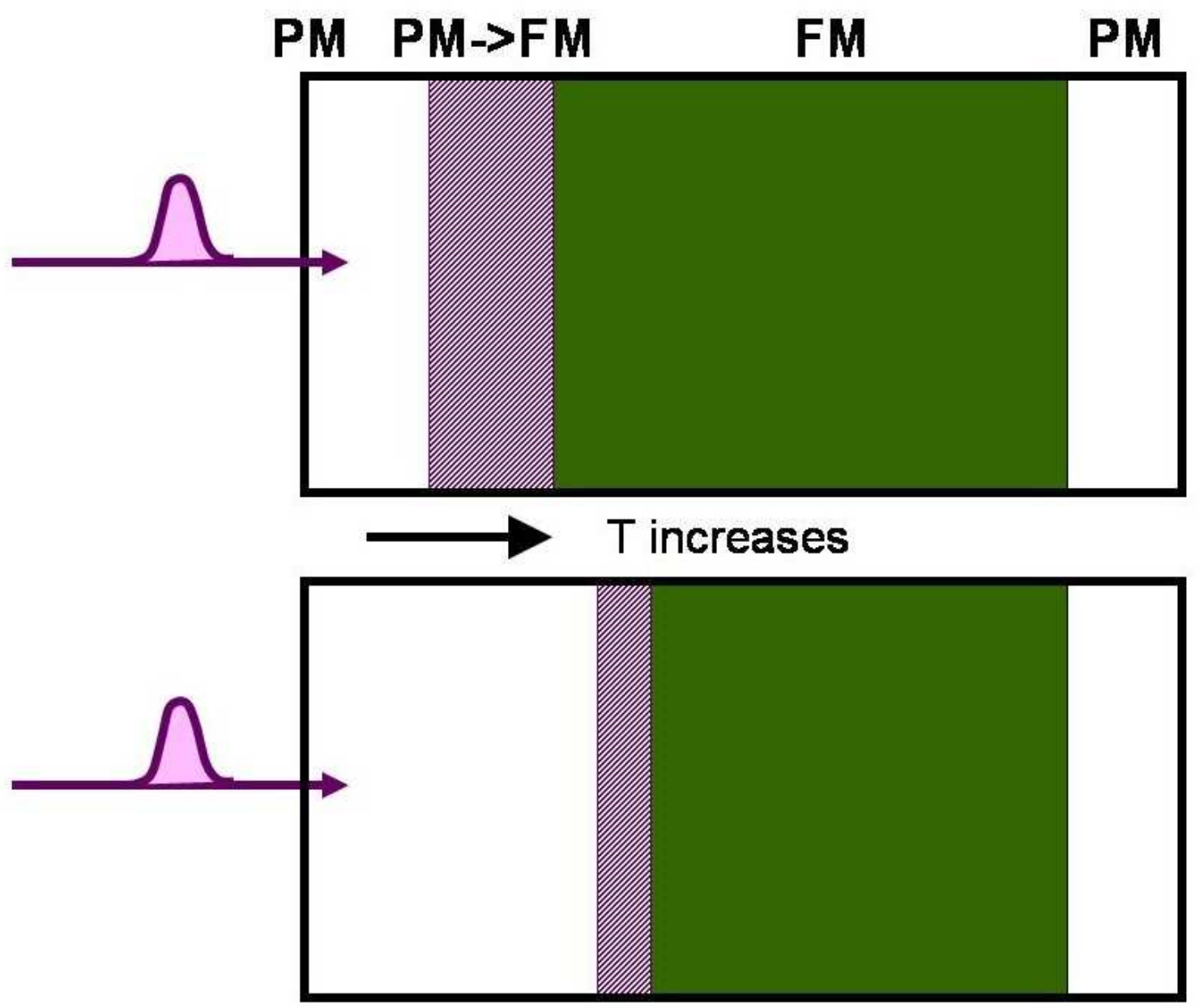

Figure 4.23: Schematics of top layer phase transition in II-PLM (Ga,Mn)As: light purple shaded areas indicate the PM $\rightarrow$ FM transition layer. As the temperature increases (from the top to the bottom panel), the photoexcited holes have a smaller effect on the closeto-transition, higher $\mathrm{T}_{C}$ regions deeper in the sample. As a result, the layer that actually undergoes the $\mathrm{PM} \rightarrow \mathrm{FM}$ transition is substantially narrower, and $\Delta \mathrm{M}$ is substantially smaller.

Calculations to verify this hypothesis require estimates of $\mathrm{T}_{C}$ vs. depth as a function of $\mathrm{Mn}$ and hole concentration, as well as estimations of $\Delta T_{C}$ increase vs. depth as a function of pre-existent conditions and photoexcited holes. According to [48, 53], experimental measurements of $\mathrm{T}_{C}$ as a function of hole density in a set of consistently 
annealed samples of $\mathrm{Ga}_{1-x} \mathrm{Mn}_{x}$ As with a wide range of $\mathrm{Mn}$ content and of various thickness, show the empirical relationship $\mathrm{T}_{C} \sim p^{1 / 3}$, independent of other physical parameters such as the Mn content or sample thickness:

$$
T_{C}=40 K\left(\frac{p}{3 \cdot 10^{19} \mathrm{~cm}^{-3}}\right)^{0.3133}
$$

However, to estimate $\mathrm{p}$ we use the Mn concentration profile and assume a constant $\mathrm{x} / \mathrm{p}$ ratio of $\sim 40 \%$. The photoexcited hole density vs. depth can be calculated using eqn. 3.11, $\Delta p=n_{0} e^{-\alpha d}$, and transient $\Delta \mathrm{T}_{C}$ increases calculated using eqn. 4.5 and the new hole density, $p+\Delta p$. We thus estimate local $\mathrm{T}_{C}$ before and after photoexcitation. When calculating $\Delta \mathrm{M}(\mathrm{T})$, we only consider the layers that undergo the $\mathrm{PM} \rightarrow \mathrm{FM}$ transition (i.e., $\mathrm{T}_{C}<\mathrm{T}<\left(\mathrm{T}_{C}+\Delta \mathrm{T}_{C}\right)$, where $\mathrm{T}$ is the operating temperature), and layers which are already in a FM state.

Though secondary in magnitude, the enhancement of FM order of layers already FM can also be estimated from $\mathrm{T}_{C}$ and $\Delta \mathrm{T}_{C}$. We assume that the local saturation magnetization per layer, $m_{0}$, is linearly proportional to the local Mn concentration $m_{0}(x) \propto x$. We calculate the proportionality constant using the known $\mathrm{M}(\mathrm{T}), \mathrm{Mn}$ concentration $\mathrm{x}$, and calculated $\mathrm{T}_{C}$ vs. depth profiles: $m_{0}(x)=0.84 \cdot 10^{-22} x$. We also assume that the shape of the local magnetization curve $m\left(\mathrm{~T}, \mathrm{~T}_{C}\right)$ is identical to the shape of the macroscopic magnetization curve $\mathrm{M}(\mathrm{T})$ - which we fit with a second order polynomial:

$$
m\left(T, T_{C}\right)=m_{0}\left[1-0.73 \frac{T}{T_{C}}-0.279\left(\frac{T}{T_{C}}\right)^{2}\right]
$$

If we assume that hole concentration follows linearly the Mn concentration and calculate the enhanced magnetization versus temperature, $\Delta \mathrm{M} / \mathrm{M}(\mathrm{T})$, the simulation does 
not match our data - see figure 4.24. The maximum $\Delta \mathrm{M} / \mathrm{M}$ is on the order of $0.5 \%$, just like in the LT-MBE grown sample, and occurs nearby $\mathrm{T}_{C}$. This is mainly because this $p \propto x$ dependence renders the sample FM at the surface for all temperatures below $20 \mathrm{~K}$, and the $\mathrm{M}$ enhancement comes exclusively from strengthening of the FM order, not from a $\mathrm{PM} \rightarrow$ FM transition.

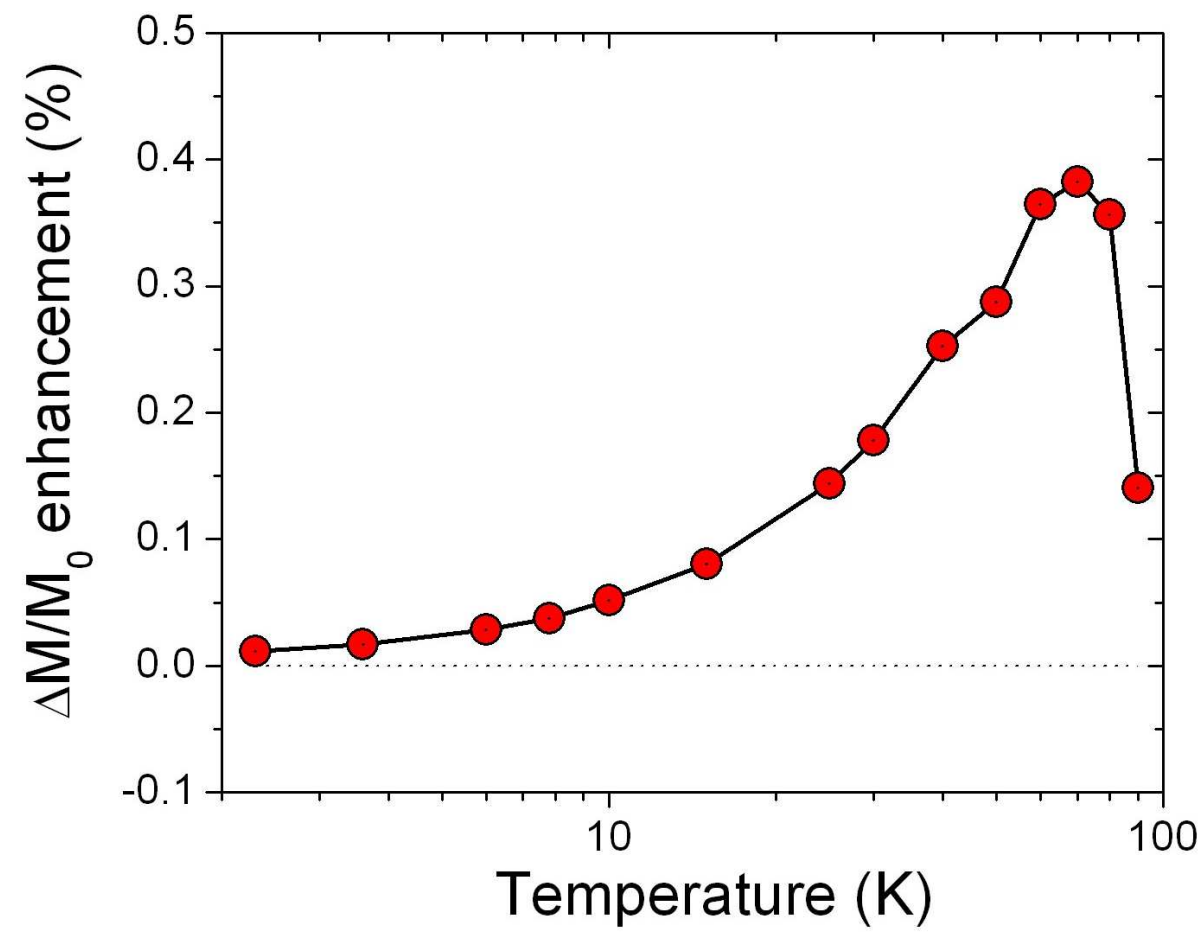

Figure 4.24: Simulation of $\Delta \mathrm{M} / \mathrm{M}(\mathrm{T})$ enhancement in II-PLM (Ga,Mn)As for $p \propto x$ show modest values at low temperatures (since most of the FM sample is close to magnetization saturation), and a maximum close to the critical point $\mathrm{T}_{C}$.

However, it is highly plausible that the holes actually segregate in the high $\mathrm{Mn}$ concentration regions due to a bending of the energy bands by the Mn presence. In this case, $p \propto x^{11}$ simulations (Fig. 4.25) yield data very close to the experimental values (Fig. $4.22(\mathrm{a}))$. In fact, at low temperatures we see a giant $\Delta \mathrm{M}$ enhancement of $\sim 10 \%$, rapidly decreasing to $\sim 5 \%$ by $10 \mathrm{~K}$. Unlike in our experiment, the enhancement is not completely 
quenched at $20 \mathrm{~K}$, but continues to decrease at a slower pace up to $25 \mathrm{~K}$, then again quickly to under $1 \%$ by $40 \mathrm{~K}$.

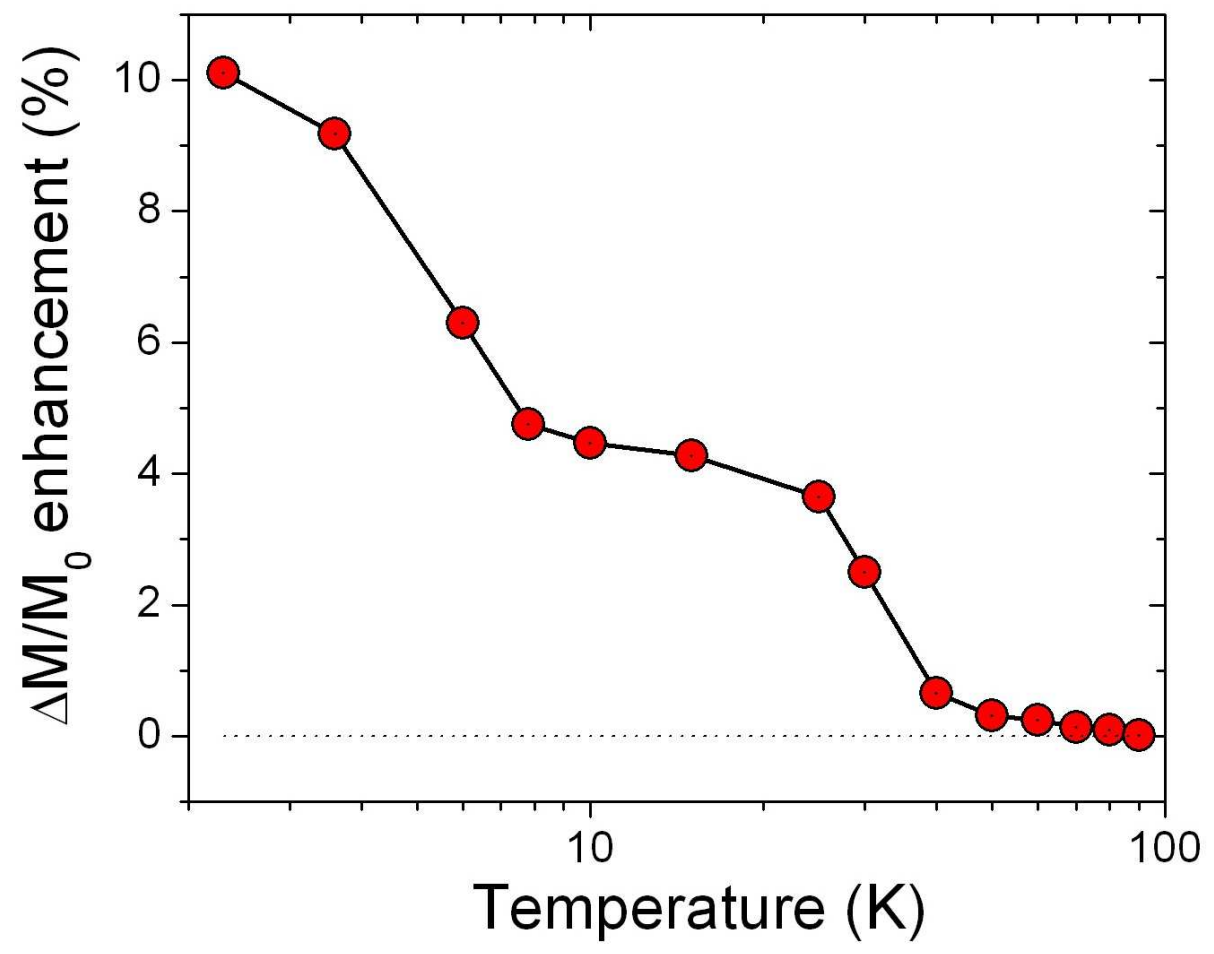

Figure 4.25: Simulation of $\Delta \mathrm{M} / \mathrm{M}(\mathrm{T})$ enhancement in II-PLM (Ga,Mn)As for $p \propto x^{11}$. Giant magnetization enhancement is observed at low temperatures $(\sim 10 \%)$, decreasing quickly with temperature up to $10 \mathrm{~K}$. Between $10 \mathrm{~K}$ and $25 \mathrm{~K}$, the $\Delta \mathrm{M} / \mathrm{M}$ falls off slower, then quickly drops off to under $1 \%$ by $40 \mathrm{~K}$.

Though our simulations give a reasonable fit for the phenomena we observe, it is possible that there are some other important effects that occur around $20 \mathrm{~K}$, which would explain the differences in the $10 \mathrm{~K}-40 \mathrm{~K}$ temperature range. For example, the quasi-2D confinement of Mn spins in the magnetic layer (Fig. 4.22(c)) could be responsible for a change in spin scattering mechanism, as indicated by transport measurements (Fig. 4.22(b)), which show an insulator-to-metal transition around $20 \mathrm{~K}$. Though not visible in the static magnetization curve, the dynamic magnetic response of the system seems to be 
sensitive to the carrier mobility decrease above $10-15 \mathrm{~K}$. This could explain the different dynamic response of Mn spins to photoexcitation of transient holes above this temperature, and could shed new light on the interpretation of the controversial transport measurements.

\subsubsection{Ferromagnetic phase enhancement in co-doped samples}

Samples D and E were also studied for effects of $(\mathrm{Ga}, \mathrm{Mn})(\mathrm{As}, \mathrm{X})$ co-doping on FM phase enhancement. In $(\mathrm{Ga}, \mathrm{Mn})(\mathrm{As}, \mathrm{P})$ sample $\mathrm{D}$, the $\mathrm{P}$ induces tensile stress in the lattice, which changes the easy axis direction to a perpendicular direction with respect to the sample plane. Figure 4.26 presents dynamic magnetization amplitude traces in sample D at representative temperatures ranging from $3 \mathrm{~K}$ to $70 \mathrm{~K}$. Since the $1 \mathrm{~T}$ external magnetic field applied to the sample renders the easy axis direction irrelevant, we do not observe major differences to the data obtained on sample C. A rather large magnetization enhancement is observed at small temperatures (Fig. 4.27), quickly quenched by $30 \mathrm{~K}$. The differences to the sample $\mathrm{C}$ magnetization dynamics consist of a smaller absolute magnetization enhancement and a longer timescale for the magnetization buildup ( $\sim 800$ ps vs. $\sim 300$ ps). This could be tied to localization of the carriers due to $\mathrm{P}$-doping.

In $(\mathrm{Ga}, \mathrm{Mn})(\mathrm{As}, \mathrm{S})$ sample $\mathrm{E}, \mathrm{S}_{A s}$ acts as a donor, compensating the $\mathrm{p}$-doping of $\mathrm{Mn}_{G a}$. As a consequence, the hole concentration in this sample is considerably reduced. Also, the $\mathrm{S}$ doping profile is much flatter than the Mn doping profile, implying that the compensation is not uniform - rather, at the tails of the Mn distribution, the sample is completely compensated, while it is less compensated in the higher Mn density regions. In figure 4.28, we present the transient magnetization amplitude dynamics in sample E. For temperatures from $4.2 \mathrm{~K}$ to above $\mathrm{T}_{C}$, no distinct photoenhancement is observed. Only the 


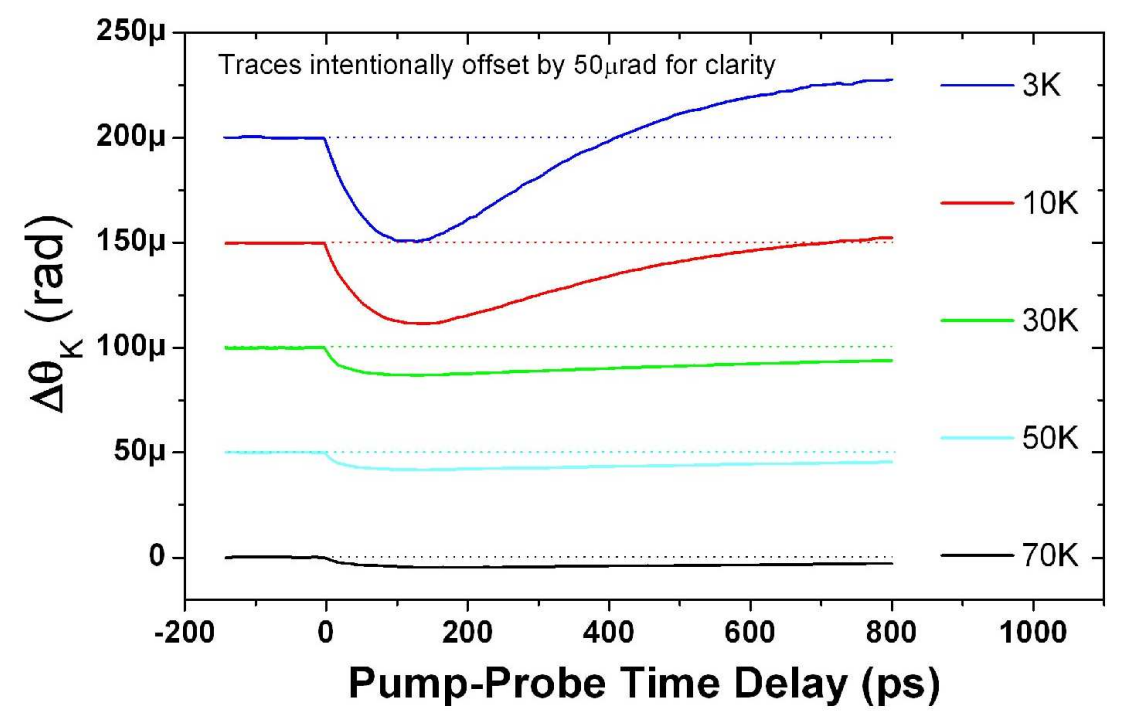

Figure 4.26: Photo-enhanced FM phase in sample $\mathrm{D}, \mathrm{Ga}_{1-x} \mathrm{Mn}_{x} \mathrm{As}_{1-y} \mathrm{P}_{y}$, shows a large magnetization enhancement at small temperatures, quickly quenched by $30 \mathrm{~K}$.

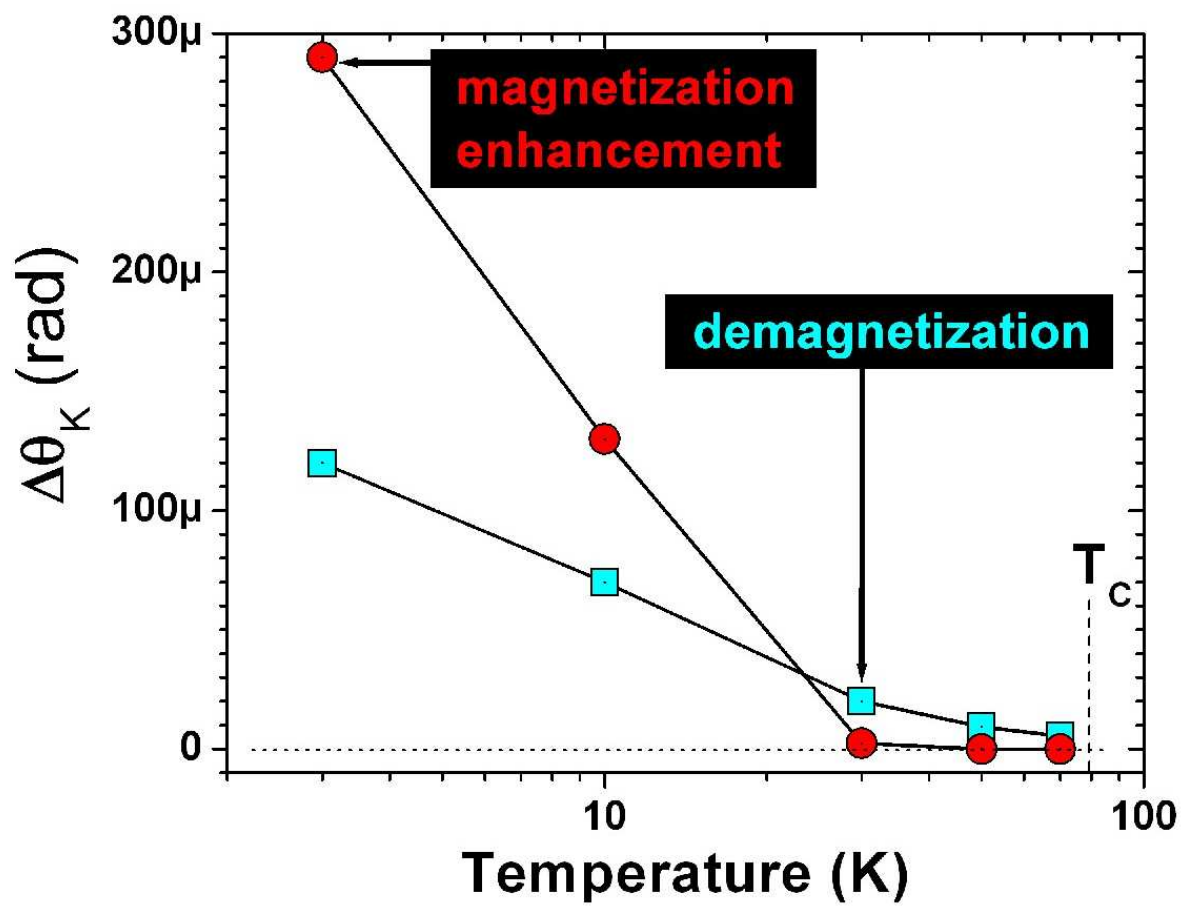

Figure 4.27: In sample D, the magnitude of magnetization enhancement (red circles) shows the same unexpected quenching behavior as in sample $\mathrm{C}$, while the demagnetization component (blue squares) disappears around $\mathrm{T}_{C}$ of $80 \mathrm{~K}$. 
very low temperature traces show a complete recovery of the initial demagnetization within the maximum 1 ns timescale given by our delay stage. This is not surprising given that in sample $\mathrm{C}$ the giant FM phase enhancement came from surface layer undergoing $\mathrm{PM} \rightarrow \mathrm{FM}$ transition. Since in sample E the surface layers are completely compensated, as discussed above, the photoexcitation of holes is not enough to support the creation of a FM phase.

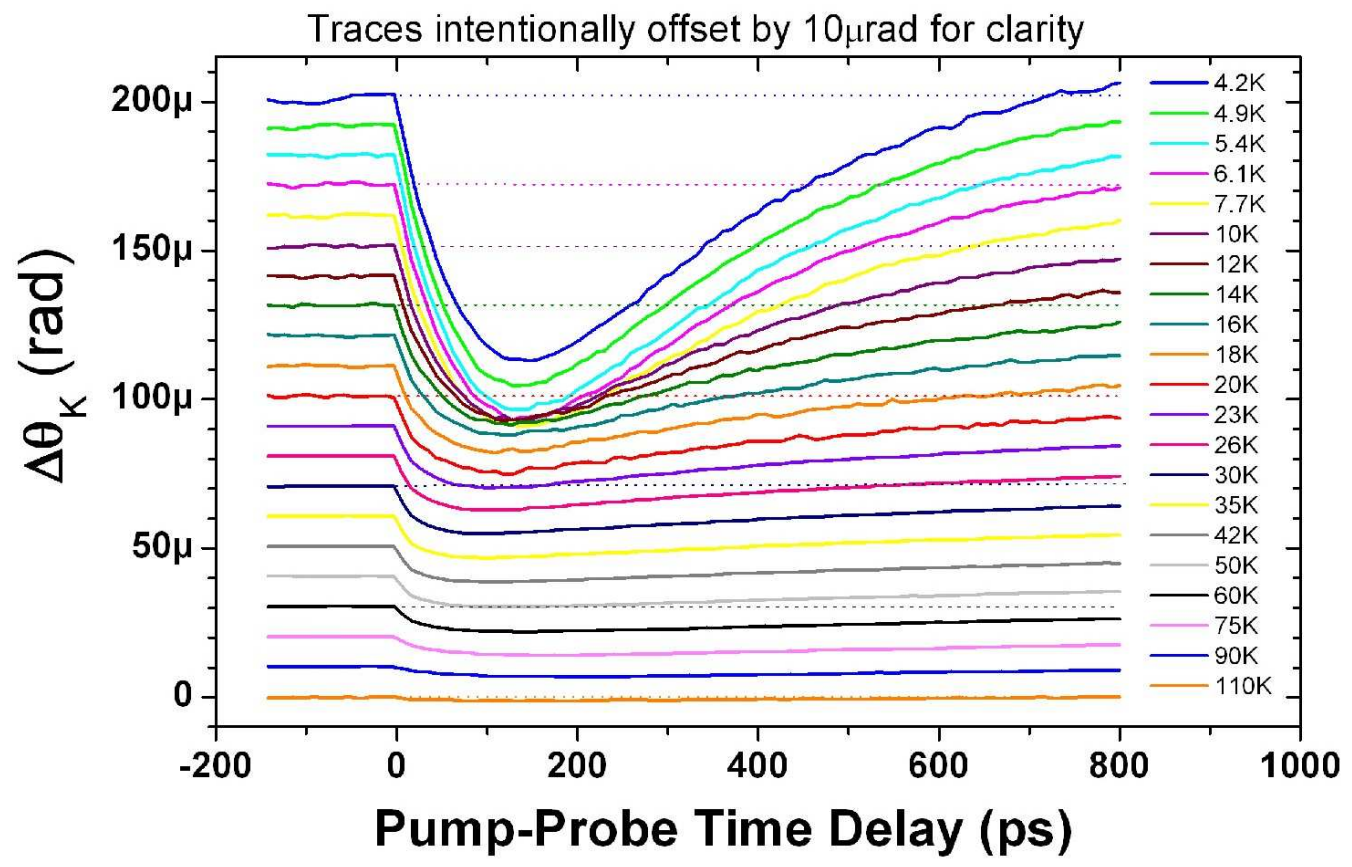

Figure 4.28: Time-resolved MOKE signals in sample E, $\left(\mathrm{Ga}_{1-x} \mathrm{Mn}_{x} \mathrm{As}_{1-y} \mathrm{~S}_{y}\right)$, for different temperatures. A buildup of the FM phase is not apparent in this sample. 


\subsection{Conclusion}

We have observed ultrafast photoenhanced ferromagnetism in LT-MBE grown thin film GaMnAs. Our data clearly show that the dynamic magnetization buildup occurs on a 100 ps time scale and exhibits a maximum below $\mathrm{T}_{C}$. Our analysis and theoretical simulations, based on $H_{p d}$ interaction between photoexcited holes and Mn spins, explain the salient features of the experimental observations, demonstrating in particular a correlation-induced peak below $\mathrm{T}_{C}$ around $20 \mathrm{~K}$ and a transient $\sim 1 \mathrm{~K}$ increase of $\mathrm{T}_{C}$. We have also observed transient signatures of an ultrafast photoinduced phase transition, resulting from the transient strengthening of the Mn-hole correlation via photoexcited holes. Exploring the magnetic field dependence of the transient magnetization enhancement, we find that at ultrafast time scales a small but finite magnetic field is needed to ensure single domain growth, in order to observe the magnetic phase transition.

In quasi-2D LT-MBE (Ga,Mn)As, in search for a considerable stronger enhancement of FM, we surprisingly find an unexpected transition around $72 \mathrm{~K}$ (considerably below $\mathrm{T}_{C}$ ). This transition is only visible in the dynamical magneto-optical properties of the system, but not in the static ones, and underlies very interesting aspects of the charge-spin interplay. We tentatively attribute it to a half-metal to metal transition.

In II-PLM GaMnAs, we have observed giant ultrafast photoenhancement of ferromagnetism. Our data show a dynamic buildup of the FM order on a 300 ps timescale. At temperatures below $20 \mathrm{~K}$, the magnitude of the enhancement is on the order of $10 \%$, much larger than in the LT-MBE grown sample. However, the amplitude of the dynamic magnetization enhancement is quickly quenched and disappears above $20 \mathrm{~K}$. This differs 
from the initial demagnetization component, which follows the static magnetization curve. We interpret the observed giant photoenhanced ferromagnetism to be due to a PM-to-FM phase transition taking place in the top layers of the sample. Simulations based on this model yield a very fit to the experimental data. In the $15 \mathrm{~K}$ to $40 \mathrm{~K}$ temperature range, we suggest that the dynamic enhancement of ferromagnetism is hindered by a decrease in the carrier mobility. II-PLM samples of (Ga,Mn)As co-doped with P or S were further studied to extend our understanding of magnetization dynamics. In particular, selectively decreasing the hole concentration in the regions of low local $\mathrm{T}_{C}$ (that would normally undergo phase transition) effectively quenches the giant enhancement of ferromagnetism observed in the non-compensated sample $\mathrm{C}$.

Our measurements reveal new fundamental transient collective magnetic processes on ultrafast time scales in $(\mathrm{Ga}, \mathrm{Mn}) \mathrm{As}$ and identify the critical role of the non-thermal Mn-hole exchange correlation for this photoinduced cooperative behavior. The new functionalities in the sub-ns timescale reported here provide exciting prospects for studying competing phases in strongly correlated magnetic materials and may open future opportunities for high-speed spin-photon-charge integrated devices. 


\section{Chapter 5}

\section{Coherent Magnetization Dynamics}

\section{and Magnetic Memory Effects}

\subsection{Outline}

(Ga,Mn)As shows rich magnetic memory effects, i.e., four-state magnetic memory, and exhibits "giant" magneto-optical and magneto-transport effects, allowing for ultrasensitive magnetic memory readout $[87,43]$. However, all demonstrated detection schemes so far have been static measurements. Achieving an understanding of collective magnetic phenomena at the fs time scale is critical for terahertz detection of magnetic memory, and essential for developing realistic "spintronic" devices and large-scale functional systems. To this end, we investigate the coherent magnetization dynamics in LT-MBE-grown (Ga,Mn)As and search for magnetic memory effects that allow for magnetic memory readout at the fs timescale. As will be shown, we uncover both thermal and non-thermal effects. 


\subsection{Ultrafast detection of magnetic memory states}

In the following, we present our findings on photoinduced femtosecond collective magnetization rotation, which allows for femtosecond detection of magnetic memory in GaMnAs [93]. We performed time-resolved polar MOKE spectroscopy with the UV pump beam at $3.1 \mathrm{eV}$ linearly polarized with peak fluences on the order of $\simeq 10 \mu \mathrm{J} / \mathrm{cm}^{2}$. The NIR beam at $1.55 \mathrm{eV}$ was used as a probe. We used this pump-probe MOKE technique to detect the out-of-plane magnetization component dynamics $\Delta M_{z}$. Intriguingly, we found that our technique directly reveals four-state magnetic hysteresis via 1 quasi-instantaneous response in the magnetization rotation. We were able to clearly identify an initial temporal regime of the magnetization rotation within the first $200 \mathrm{fs}$, during photo-excitation and the highly non-equilibrium, non-thermal carrier redistribution times. We attributed this to a carrier-mediated magnetic field pulse, arising without assistance from either lattice heating or demagnetization.

Our sample A structure exhibits four-state magnetic memory functionality, as shown in figure 5.1. By sweeping an external magnetic field B aligned nearly perpendicular to the sample normal, but with small components in both the $\mathrm{X}$ and the $\mathrm{Y}$ directions in the sample plane, one can sequentially access four magnetic states $\mathrm{X}+\rightarrow \mathrm{Y}-\rightarrow \mathrm{X}$ $\rightarrow \mathrm{Y}+$ by abrupt $90^{\circ} \mathrm{M}$ switching between the $\mathrm{XZ}$ and $\mathrm{YZ}$ planes (Fig. 5.1(a)). In these states, $\mathrm{M}$ aligns along a direction arising as a combination of the external magnetic field and the anisotropy field along the in-plane easy axes ([100] and [010]). The multistep magnetic switching manifests itself as abrupt jumps in the four-state hysteresis of Hall magneto-resistivity $\rho_{\text {Hall }}$ at coercivity fields $\mathrm{B}_{c 1}$ and $\mathrm{B}_{c 2}$ (Fig. 5.1(b)) (the planar Hall 

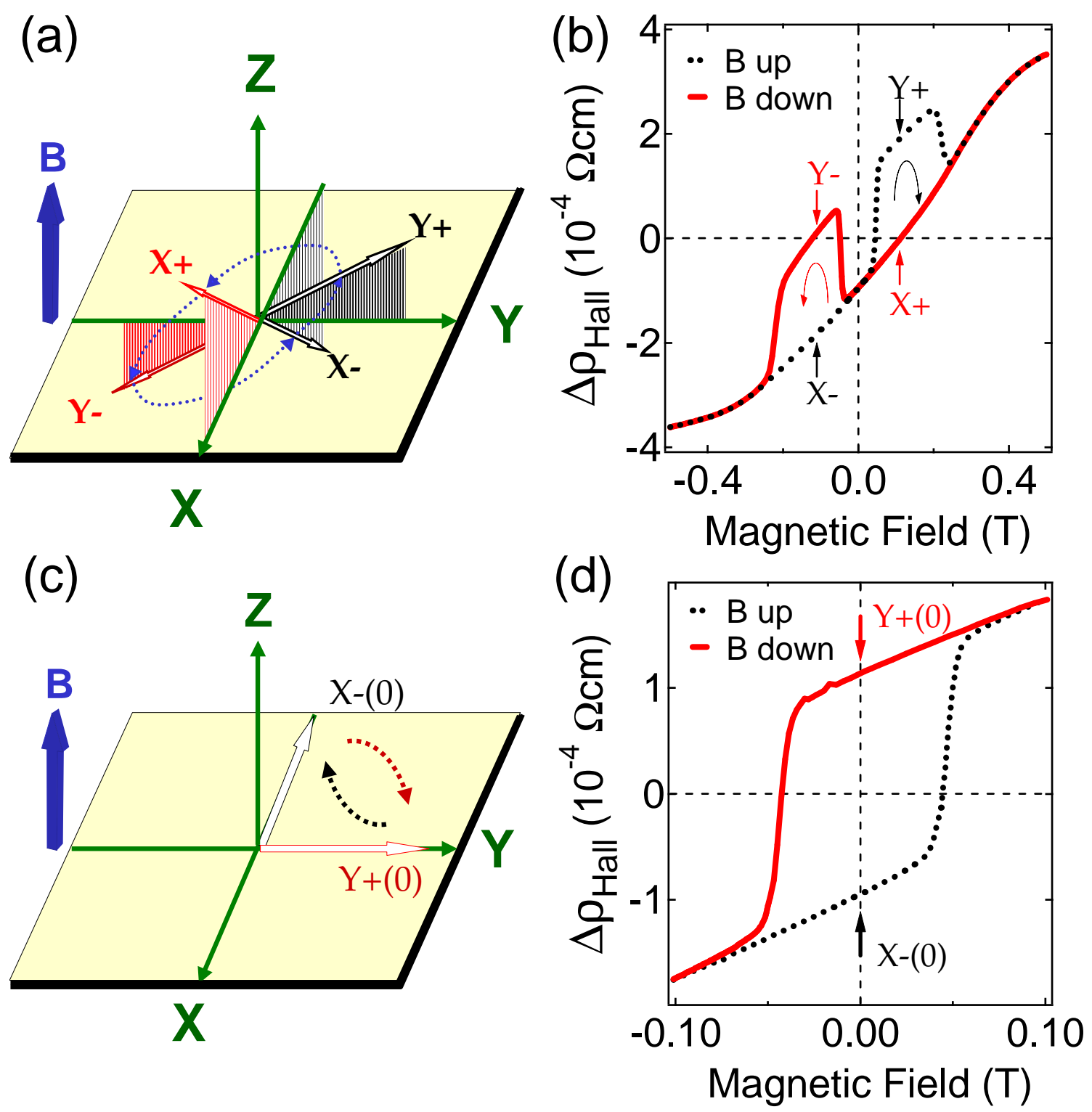

Figure 5.1: (a)-(b): Sweeping a slightly tilted B field ( $5^{\circ}$ from the Z-axis and $33^{\circ}$ from the Xaxis) up (dash) and down (solid) leads to consecutive $90^{\circ}$ magnetization switching between the XZ and YZ planes, manifesting as a "major" hysteresis loop in the Hall magnetoresistivity. (c)-(d): The "minor" hysteresis loop with B field sweeping in the vicinity of $0 \mathrm{~T}$. The magnetic memory state $\mathrm{X}-(0)$ or $\mathrm{Y}+(0)$ is parallel to one of the easy axis directions in the XY plane. 
effect [51]). The continuous slopes of $\rho_{\text {Hall }}$ indicate a coherent out-of-plane M rotation during the perpendicular magnetization reversal (the anomalous Hall effect [60]). Figure 5.1(c)-(d) show the B scans in the vicinity of $0 \mathrm{~T}$, with the field turning points between the coercivity fields, i.e., $\mathrm{B}_{c 1}<|\mathrm{B}|<\mathrm{B}_{c 2}$. This produces a "minor" hysteresis loop, accessing two magnetic memory states at $\mathrm{B}=0 \mathrm{~T}$ : $\mathrm{X}-(0)$ and $\mathrm{Y}+(0)$.

Figure 5.2(a) shows the magnetic field scan traces of photoinduced $\Delta \theta_{K}$ at three time delays, $-1 \mathrm{ps}, 600 \mathrm{fs}$ and $3 \mathrm{ps}$, respectively. $\Delta \theta_{K}$ is negligible at $\Delta \mathrm{t}=-1 \mathrm{ps}$ since the pump pulse has not excited the sample yet. However, a mere 600 fs after photo-excitation, a clear photoinduced four-state magnetic hysteresis is observed, with four abrupt switchings, arising as a direct consequence of magnetic memory effects. Four distinct magnetic states $\mathrm{X}+, \mathrm{X}-, \mathrm{Y}-, \mathrm{Y}+$ for $|B|=0.2 \mathrm{~T}$ give different $\Delta \theta_{K}$ signals, as marked in figure 5.2(a).

It is critical to note that the steady-state MOKE curve, without the pump laser excitation, doesn't show any sign of magnetic switching or memory behavior (right inset, Fig. 5.2(a)). Furthermore, the B field scans also show a saturation behavior at a relatively high field $|B|>0.6 \mathrm{~T}$, which we will discuss later. In addition, the photo-induced hysteresis loops at $\Delta \mathrm{t}=3 \mathrm{ps}$ sustain similar shapes, with only slightly larger amplitudes than those at $\Delta \mathrm{t}=600 \mathrm{fs}$, indicating that the dynamic magnetic processes responsible for the abrupt jumps occur on a femtosecond time scale.

Figure 5.2(b) presents the photoinduced $\Delta \theta_{K}$ dynamics for the four initial states $\mathrm{X}+, \mathrm{X}-, \mathrm{Y}-$, and $\mathrm{Y}+$. There is an extremely fast photo-induced $\Delta \theta_{K}$ within $200 \mathrm{fs}$, whose magnitude and sign distinctly differ, depending on the initial prepared state, consistent with figure 5.2(a). Particularly, the substantial difference in the $\Delta \theta_{K}$ under the same B field - 

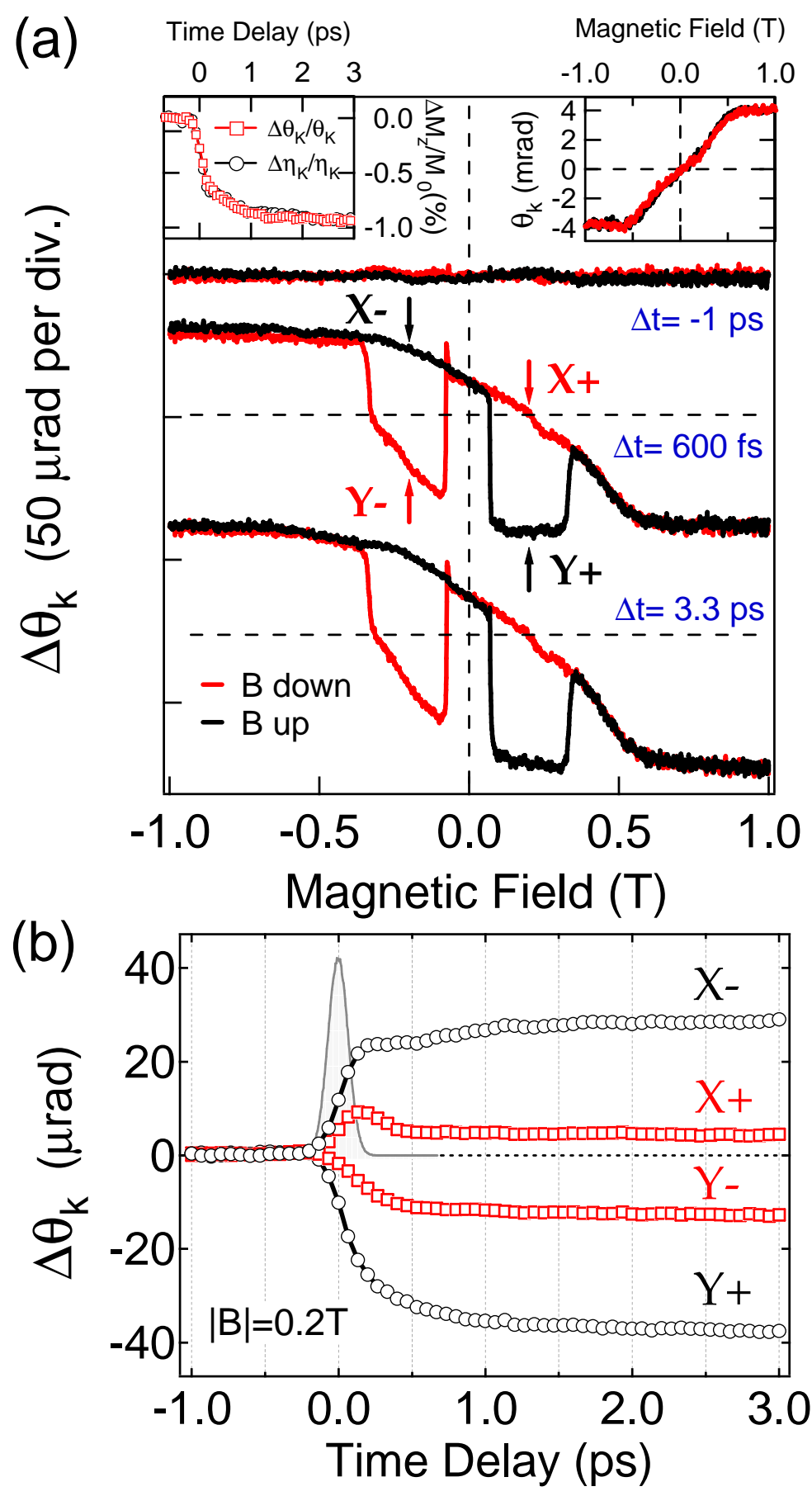

Figure 5.2: (a) Magnetic field scans of $\Delta \theta_{K}$ at $5 \mathrm{~K}$ for three time delays: $\Delta \tau=-1 \mathrm{ps}, 600 \mathrm{fs}$, and 3.3 ps. The traces are vertically offset for clarity. Inset (left): the temporal profiles of normalized Kerr $\left(\theta_{K}\right)$ and ellipticity $\left(\eta_{K}\right)$ angle changes at $1.0 \mathrm{~T}$; Inset (right): the static magnetization curve at $5 \mathrm{~K}$. (b): Temporal profiles of photoinduced $\Delta \theta_{K}$ for the four distinct magnetic states for $|B|=0.2 \mathrm{~T}$. The shaded area is the pump-probe cross-correlation. 
for instance between the $\mathrm{X}+$ and $\mathrm{Y}+$ states - shows that the magnetic dynamics contains contributions beyond demagnetization $[97,96,7]$.

The photoinduced dynamics of the zero-field memory states (Fig. 5.1(c)) elucidates the salient features of the femtosecond dynamic magnetization processes. Figure 5.3(a) shows the temporal profiles of the photo-induced $\Delta \theta_{K}$ for the $\mathrm{X}-(0)$ and $\mathrm{Y}+(0)$ states. These are "pure" magnetic memory states, since the magnetic field used to prepare the magnetization direction is now turned off. Since the initial magnetization vector is strictly in the sample plane, $\Delta \theta_{K}$ in the first 200 fs clearly reveals out-of-plane spin rotation (with negligible contribution from demagnetization). More intriguingly, the $\mathrm{M}$ in the $\mathrm{X}-(0)$ and $\mathrm{Y}+(0)$ states rotates to different Z-axis directions, as illustrated in figure 5.3(b), which leads to opposite signs of the photo-induced signals (and is responsible for the observed four-state magnetic switchings).

Furthermore, the observation of an initial discontinuity in the temporal profiles of the $\mathrm{M}$ tilt reveals two distinct temporal regimes, as marked in figure 5.3(a): a substantial magnetization rotation concluded after the first $200 \mathrm{fs}$, followed by a much slower magnetization rotation change afterwards.

Next, we discuss the origin of the femtosecond magnetization rotation effect. In the previously held picture of optically excited magnetization rotation in ferromagnetic materials, the photo-excitation alters the anisotropy fields via quasi-equilibrium mechanisms such as heating of the lattice (magneto-crystalline anisotropy) or heating of the spins (shape anisotropy) [90]. Since the in-plane magnetic memory states (Fig. 5.1(c)) have negligible shape anisotropy, an effective B field can only occur on a time scale of several picoseconds via 


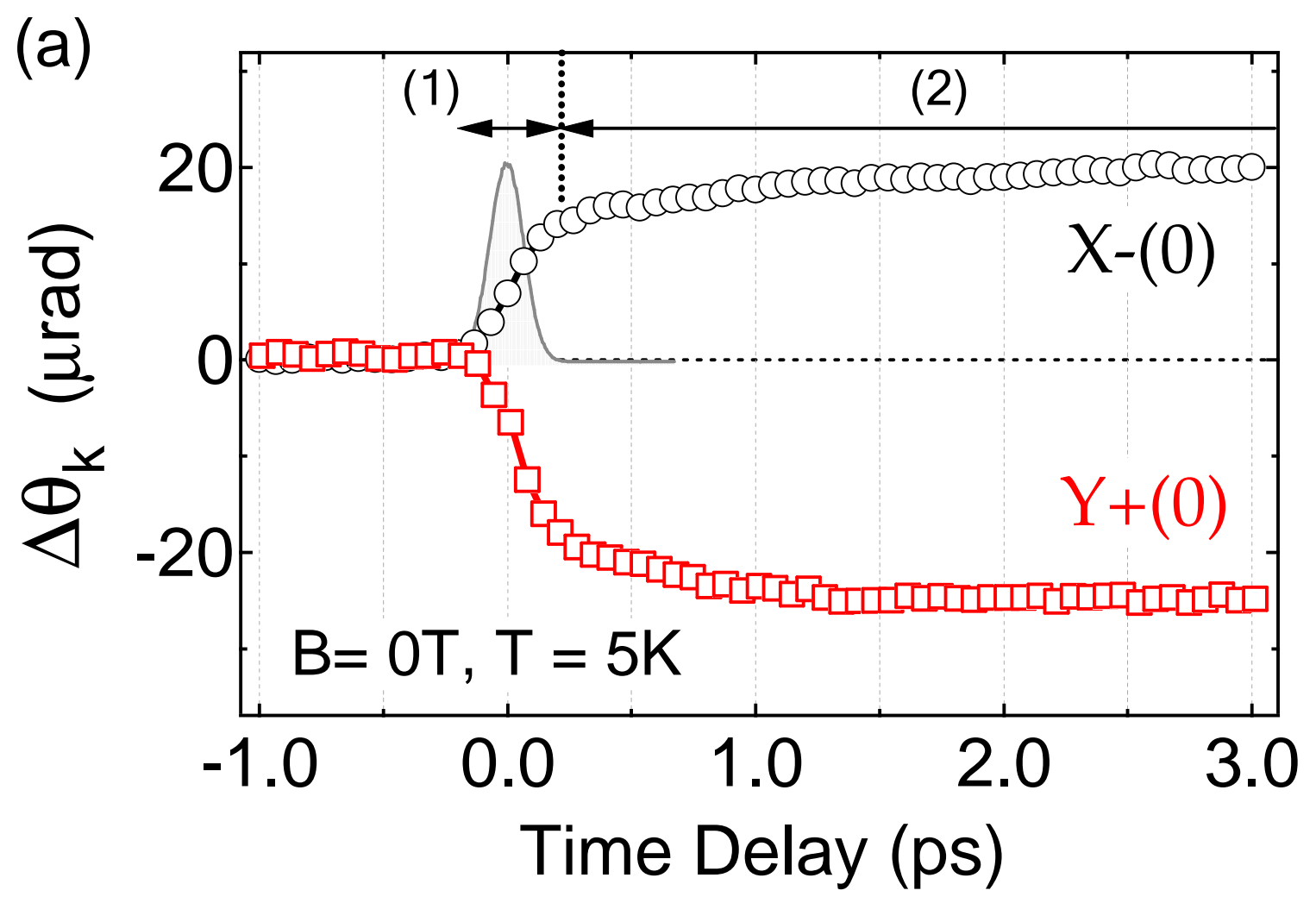

(b)

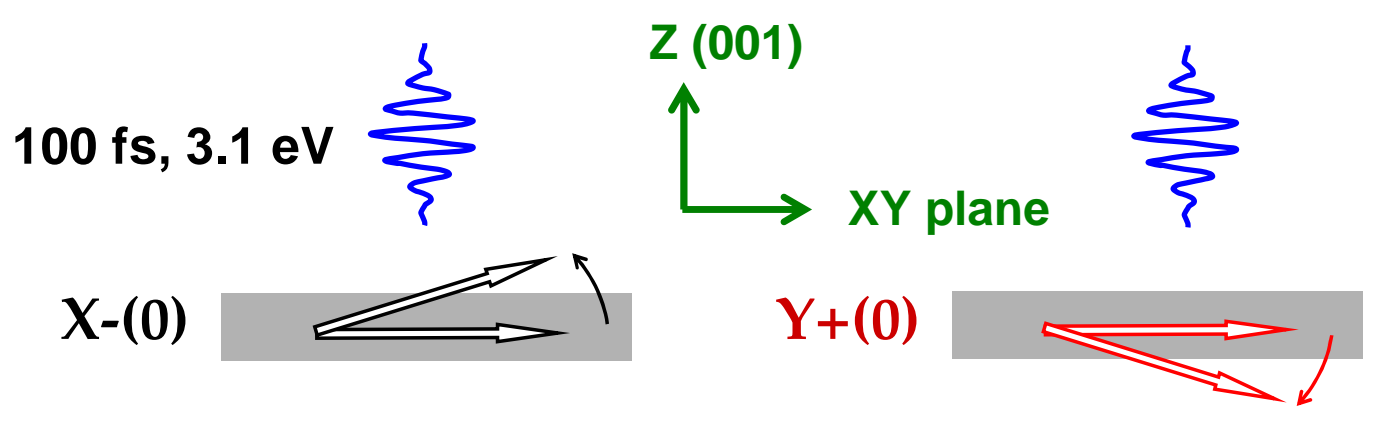

Figure 5.3: (a) The photo-induced $\Delta \theta_{K}$ for two in-plane magnetic memory states, showing two regimes of $\mathrm{M}$ rotation. The shaded area is the pump-probe cross-correlation. The opposite, out-of-plane M rotations for $\mathrm{X}-(0)$ and $\mathrm{Y}+(0)$ states are illustrated in (b). 
the lattice heating mechanism. However, it has been shown theoretically [16] that the Mn spin in GaMnAs can respond quasi-instantaneously to a femtosecond effective magnetic field pulse generated by the photoexcited hole spin and second-order nonlinear optical excitation. Such an effective magnetic field pulse may be thought as a femtosecond modification of the magnetic anisotropy field during the photo-excitation [17]. To describe the realistic system, one needs to treat microscopically the magnetic anisotropy from the complex valence band structure and non-thermal carrier distribution in the initial femtosecond regime, which strongly affects the photoexcited carrier spins.

Due to the hole-mediated effective exchange interaction between Mn spins, the anisotropy fields in GaMnAs are a direct consequence of the coupling of several valence bands by the spin-orbit interaction. Consequently, a phenomenological understanding of the anisotropy fields reveals that they are sensitive functions of the transient hole distribution and coherences. In the static case, recent experimental $[50,30]$ and theoretical [21] investigations have shown that increasing the hole density significantly reduces the cubic anisotropy $\left(\mathrm{K}_{c}\right)$ along the $[100]$ direction, while enhancing the uniaxial anisotropy $\left(\mathrm{K}_{u}\right)$ along $[1 \overline{1} 0]$. One therefore expects an effective magnetic field pulse $\left(\Delta B_{c}\right)$ along the $[1 \overline{1} 0]$ direction to be turned on by the optical excitation (Fig. 5.4(a)). The photo-triggered $\Delta B_{c}$ then exerts a spin torque on the $\mathrm{M}$ vector, $\Delta \vec{B}_{c} \times \vec{M}$, and pulls it away from the sample plane. The directions of the spin torques for the $\mathrm{X}-(0)$ and $\mathrm{Y}+(0)$ states are opposite to each other, leading to different $M$ rotation paths for the two memory states (Fig. 5.3). Since this mechanism is carrier-mediated, the appearance of $\Delta B_{c}$ is quasi-instantaneous, limited only by the pulse duration of $\sim 100$ fs [17]. This femtosecond magnetic anisotropy 
contribution from the non-thermal photoexcited carriers should be contrasted to the quasithermal changes in the magnetic anisotropy, arising from the transient lattice temperature elevation on the picosecond time scale $[66,92,31]$.
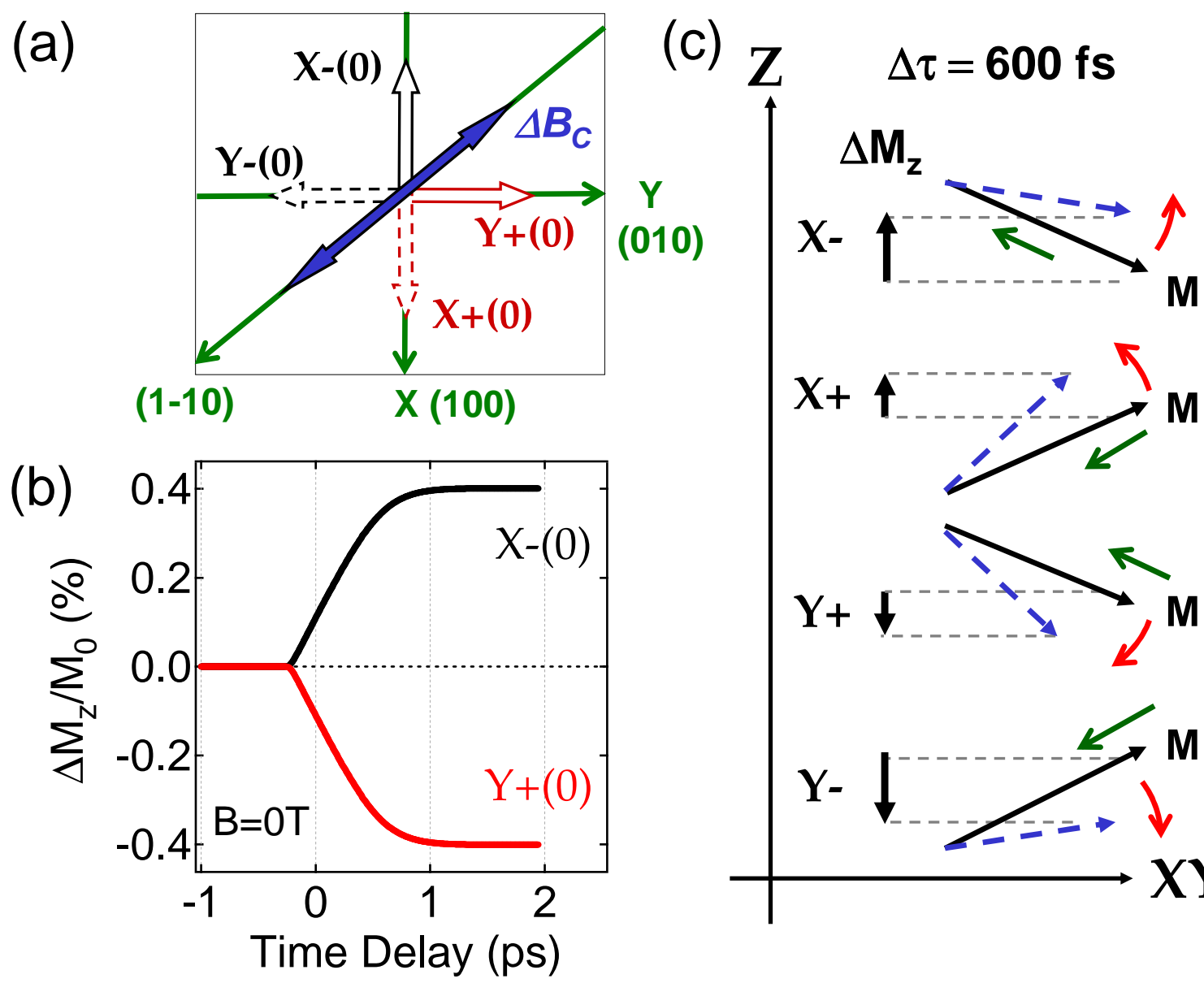

Figure 5.4: (a) Schematics of the photoexcited carrier induced anisotropy field $\Delta B_{c}$. (b) Simulations of $\Delta M_{z} / M_{0}$ for the two magnetic memory states. Parameters used in calculation are $K_{c}=1.19810^{-2} \mathrm{meV}, K_{u}=0.37310^{-2} \mathrm{meV}, K_{3}=0.74610^{-2} \mathrm{meV}, \mathrm{T}_{1}=330 \mathrm{fs}$ and $3 \%$ excess of photoexcited carriers. (c) Schematics of the photoinduced $M_{z}$ changes for the $\mathrm{X}-, \mathrm{X}+, \mathrm{Y}-$, and $\mathrm{Y}+$ states at $|B|=0.2 \mathrm{~T}$.

Next we discuss the origin of the discontinuity that separates the two temporal regimes in the collective magnetization rotation [Fig. 5.3]. The quick termination of the initial magnetization tilt implies that the effective $\Delta B_{c}$ pulse induced by the photo-excitation 
decays within the first hundreds of femtoseconds. A large $\Delta B_{c}$ requires an extensive nonthermal distribution of transient holes in the high momentum states of the valence band [21], which are not populated in the unexcited sample. This is due to the large spin anisotropy of these hole states via their strong spin-orbit interaction. In our experiment, immediately following photo-excitation at $3.1 \mathrm{eV}$, a large density of transient holes distributes themselves over almost half of the Brillouin zone along the L [111] direction. Coincidently, the Mn-hole exchange interaction is also believed to be enhanced along [111] via the strong hybridization of their orbitals [12]. Consequently, these photoexcited hole states contribute strongly to the magnetic anisotropy fields. The following rapid relaxation and thermalization of these high momentum holes to the bottom of the band, due to carrier-carrier and carrier-phonon scattering, will reduce the $\Delta B_{c}$ within a few hundred femtoseconds. The subsequent, picosecond rotation process arises from the change in magnetic anisotropy induced by the lattice temperature elevation. Our results thus directly reveal a complex scenario of collective spin rotation marked by the transition from a non-equilibrium, carrier-mediated regime $(<200 \mathrm{fs})$ to a thermal, lattice-heating regime on the ps time scale.

We modeled this effect phenomenologically [17] by deriving $\Delta B_{c}$ from the magnetic free energy,

$$
W_{\text {anis }}=-\frac{K_{c}}{S^{4}} S_{x}^{2} S^{2} y+\frac{K_{u}}{S^{2}} S_{x}^{2}+\frac{K_{3}}{S^{2}} S_{z}^{2}
$$

that describes the cubic $\left(K_{c}\right)$ and uniaxial $\left(K_{u}\right)$ contributions with the additional time dependent modification to $K_{c} / K_{u}$ from the strongly photoexcited anisotropic hole states. The corresponding contribution to the equations of motion is $\partial_{t} S=S \times F_{\text {anis }}$, where $F_{\text {anis }}=$ $-\partial W_{\text {anis }} / \partial S$. The decrease in the magnitude of $K_{c} / K_{u}$ grows with pulse duration and 
then decreases with the energy relaxation time of the high-momentum photoexcited holes $\left(\mathrm{T}_{1}\right)$. The results of our calculation are shown in figure $5.4(\mathrm{~b})$, which gives a similar time dependence of the normalized $\Delta M_{z}$ with magnitude of about $0.4 \%$ of the total magnetization $M_{0}(\sim 4 \mathrm{mrad}$ at $5 \mathrm{~K})$, which compares well with the experiment. A microscopic theoretical treatment would have to include the strong valence band spin-orbit coupling effects.

Finally, figure 5.4(c) illustrates the femtosecond detection of the four-state magnetic hysteresis shown in figure 5.2. By incorporating both the photo-induced rotation (red arrows) and the demagnetization (green arrows) effects, we can visualize different $M_{z}$ changes for the four magnetic states, consistent with our observation. Note that the demagnetization is also consistent with the high field saturation behavior in figure 5.2(a). At $|B|>0.6 \mathrm{~T}, \mathrm{M}$ is aligned almost perpendicular to the sample. Thus the photo-induced signals at these fields represent decreasing of the M amplitude, which is more or less constant with respect to the field.

Thus, the femtosecond response of the photoinduced magnetization rotation in GaMnAs allows for ultrafast detection of four-state magnetic memory. Our observations unequivocally identify a non-thermal, carrier-mediated mechanism relevant in the femtosecond magnetization rotation regime. It occurs without assistance of either lattice heating or demagnetization, and may represent an as-yet-undiscovered universal principle in all carrier-mediated ferromagnetic materials. This has especially significant potential for future applications, in particular concerning oxides, which hold promise of achieving Curie temperatures far above room temperature. 


\subsection{Nanosecond timescale coherent magnetization dynamics}

Due to the hole-mediated effective exchange interaction between Mn spins, the anisotropy fields in GaMnAs are a direct consequence of the coupling of several valence bands by the spin-orbit interaction. The lattice constant of $(\mathrm{Ga}, \mathrm{Mn}) \mathrm{As}$ is larger than that of GaAs depending on the Mn concentration, and thin film GaMnAs is tetragonally distorted along the growth direction [001] by compressive strain when grown on GaAs. The electronic structure of the hole sub-bands depend on the epitaxial strain. At high hole concentrations, for compressive strains, the in-plane component of the hole spin is greater than the perpendicular component. As a consequence, a stronger exchange interaction will occur for the in-plane orientation of the magnetization, equivalent to a larger in-plane anisotropy or an in-plane easy axis direction. Magnetic anisotropy fields are sensitive functions of not only the hole concentration and polarization, depending on their distribution in the energy bands, but also on temperature and on transient carrier effects.

In this section, we present the observation of magnetic memory effects in timeresolved coherent magnetization dynamics in GaMnAs on the ns timescale. Under zero or finite external magnetic field perpendicular to the sample plane, we observe precession of the magnetization vector around a direction arising as a combination of the external magnetic field and the in-plane anisotropy fields. These trajectories depend unambiguously on the initial magnetization state the sample is prepared in, and can be explained by changes in the anisotropy field direction due to both thermal and transient carrier effects. The initiation part of the magnetization dynamics (fast components) is attributed to changes in the anisotropy field due to photoexcited transient carriers (non-thermal effects). The 
slow components following (tens of ps) are attributed to thermal effects on the anisotropy fields. In order to assess the effect of temperature on the easy axis reorientation, we study the temperature dependence of the ferromagnetic resonance. The experiment shows a disappearance of the oscillations around $30 \mathrm{~K}$, which we associate with a change in easy axis orientation.

\subsubsection{Magnetic memory}

At low temperatures, $\mathrm{M}$ naturally aligns along one of two easy axes directions within the film plane, $\mathrm{X}$ [100] or $\mathrm{Y}$ [010]. As we have shown in the previous section, we can manipulate the orientation of $\mathrm{M}$ by adding an external magnetic field $\mathrm{B}$ almost perpendicular to the sample plane. We investigate the ns-timescale coherent magnetization dynamics under various external field and temperature conditions by measuring the polar MOKE angle $\Delta \theta_{K}(\mathrm{t})$ corresponding to changes in the magnetization component along [001], i.e. the $\hat{z}$ direction perpendicular to the sample plane.

Figure 5.5 shows the $\mathrm{M}$ dynamics at a temperature of $5 \mathrm{~K}$ and zero external field, when prepared in two different initial magnetic states both lying in the sample plane, each along one of the easy axis directions (i.e., zero-field memory states $\mathrm{X}-(0)$ and $\mathrm{Y}+(0)$ ). As shown before, figures 5.5(a)-(b) illustrate the $\rho_{\text {Hall }}$ measurements that indicate the magnetic state the system is in, depending on external magnetic field applied and sweep history. Figure 5.5(c) presents a schematics of the magnetic field pulse triggered by the pump pulse along [11̄0]. Finally, figure 5.5(d) shows that depending on which state the system is prepared in, $\mathrm{X}-(0)$ or $\mathrm{Y}+(0)$, the coherent magnetization dynamic response at the ns-timescale is strikingly different. As revealed by the ps-timescale dynamics, the 
magnetization dynamics for these two magnetic memory states is initiated very differently (Fig. 5.3(a)). This difference continues to manifest itself on the ns timescale, where large contrast oscillations of the polar MOKE angle $\Delta \theta_{K}$ indicate precession of the $\mathrm{M}$ vector around a new anisotropy field direction induced by the pump. As evident in the data, the oscillations are not symmetrical and take place around significantly different mean values.
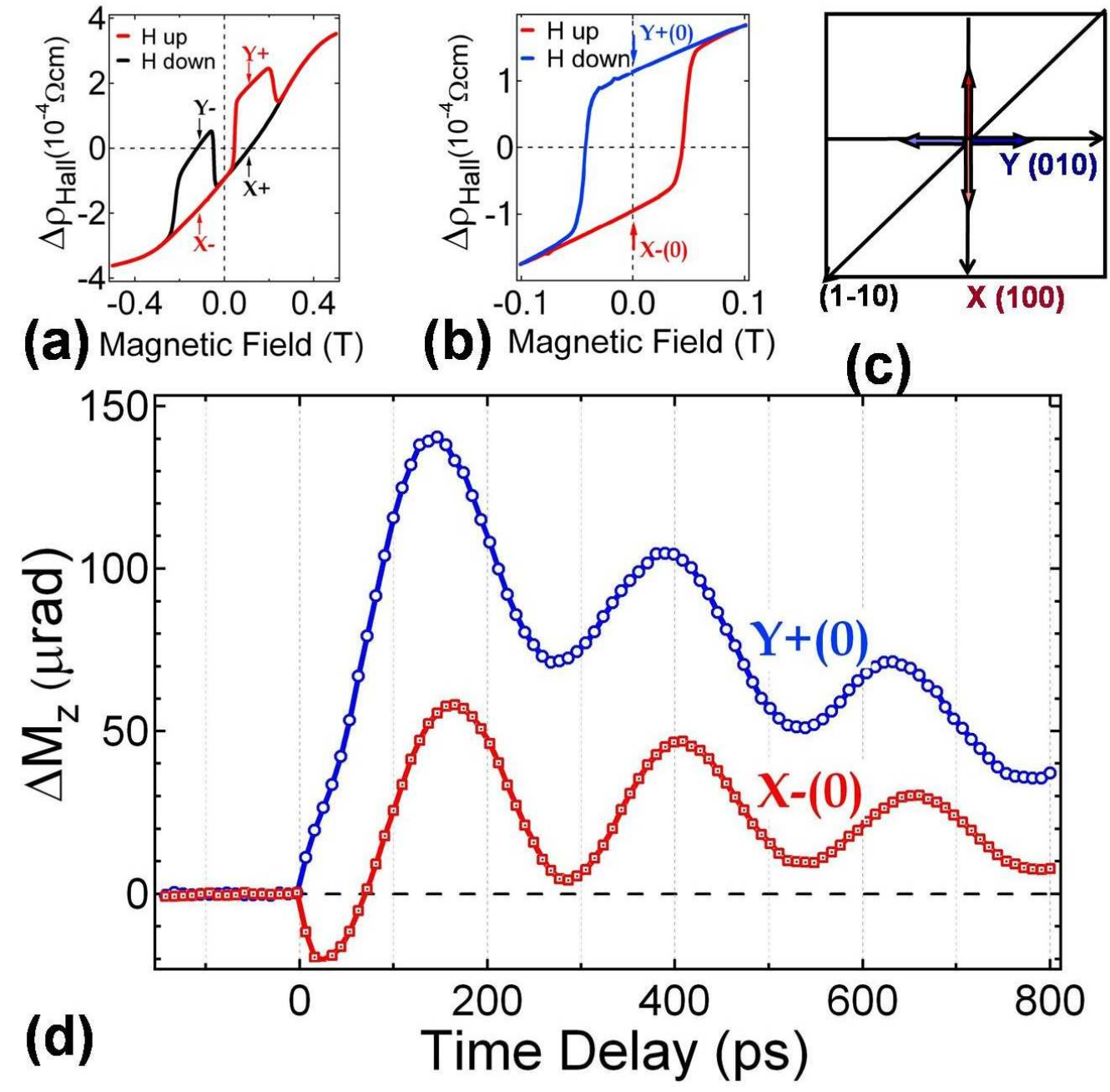

Figure 5.5: Magnetic memory as observed in coherent magnetization dynamics at the ns timescale. (a) Hall resistivity hysteresis loop. (b) Minor hysteresis loop. (c) Magnetic field pulse triggered along [110] by the pump pulse. (d) Coherent magnetization dynamics for two "pure" magnetic memory states, initiated by non-thermal effects but driven by thermal effects at the ns timescale. 
The pump arriving at the sample creates a population of transient carriers: electrons that get quickly trapped by deep defect states $\left(\mathrm{As}_{G a}\right)$, and long-lived holes. Heating of the lattice cannot be the only explanation for the magnetization behavior observed. When preparing $\mathrm{M}$ along $\mathrm{X}-(0)$ or $\mathrm{Y}+(0)$, if only thermal effects were at play, one would expect equal reorientations of these easy axes towards the [1ํㅣㄹ diagonal direction. This would kick off the M precession around the new effective field. Because of the symmetry of the two initial states with respect to [11̄0], one would expect the observed oscillations to be mirroring each other with respect to zero. What we observe instead are fast responses with opposite sign, followed by almost in-phase oscillations around different mean values of the same sign. The initial response is, as we showed in the previous section, due to a magnetic field pulse $\Delta \mathrm{B}_{c}$ turned on by the excitation of a non-equilibrium population of hot holes deep into the valence band. This magnetic field pulse is quasi-instantaneous, appears along the $[1 \overline{1} 0]$ direction, and is short lived: as soon as the holes thermalize $(\sim 1 \mathrm{ps})$ and begin to cool down, the magnetic field pulse disappears. On a longer timescale, upon release of energy from the thermalized holes to the lattice, the local temperature and hole concentration transiently increase, resulting in a change of the in-plane magnetic anisotropies and easy axis orientation again towards [1ํㅣㄹ. As a result, the effective magnetic field experienced by the Mn spins changes, thereby triggering the observed precession. The system then relaxes back to the equilibrium temperature via a slow (ns) thermal diffusion process, and the easy axis returns to the [100] direction. The M displacement due to $\Delta \vec{B}_{c} \times \vec{M}$ initially takes place along the $\hat{z}$-axis, showing quick initial opposite signs in the polar MOKE angle response. After the magnetic field pulse disappears, the now-displaced M vector begins to 
precess along the slowly varying, temperature dependent easy axis direction.

Memory effects are embedded in how the initial state is prepared. In the previous case, by following a minor hysteresis loop, we prepared $\mathrm{M}$ along the $\mathrm{X}-(0)$ or $\mathrm{Y}+(0)$ direction, leading to different magnetization dynamics. We present evidence to support the existence of memory effects by following the $\mathrm{M}$ dynamics along the entire hysteresis loop. The $\mathrm{M}$ dynamics at $5 \mathrm{~K}$ under different perpendicular external magnetic fields is shown in figures 5.6(a) $-(\mathrm{g})$. We sweep the magnetic field from $-1 \mathrm{~T}$ to $1 \mathrm{~T}$ and back to $-1 \mathrm{~T}$ (Fig. 5.6(h)), such that the in-plane magnetization component $\left(\mathrm{M}_{I P}\right)$ aligns sequentially along $\mathrm{X}+, \mathrm{Y}-$, $\mathrm{X}-$, and $\mathrm{Y}+$. The $\mathrm{M}$ dynamics illustrated corresponds to complete and almost complete alignment of $\mathrm{M}$ along the $\hat{z}$-axis at $|B|=1$ or $0.5 \mathrm{~T}$, history dependent alignment of $\mathrm{M}_{I P}$ along the in-plane easy axis directions at $|B|=0.2 \mathrm{~T}$, or total alignment of $\mathrm{M}$ along $\mathrm{X}+(0)$ or $\mathrm{X}-(0)$ at $\mathrm{B}=0 \mathrm{~T}$. One should note the difference to the figure 5.5(d) M preparation, in which we access only minor loops by sweeping the field between $-0.2 \mathrm{~T}$ and $0.2 \mathrm{~T}$, and thus have $\mathrm{M}$ lie along $\mathrm{X}+(0)$ or $\mathrm{Y}-(0)$.

At high fields, when $\mathrm{M}$ is almost completely aligned along the external field, and the small $\mathrm{M}_{I P}$ component is along $\mathrm{X}+/ \mathrm{X}-$, we see history-independent $\mathrm{M}$ dynamics, showing quick demagnetization at $|B|=1 \mathrm{~T}$, as well as rapid quenching of the oscillations at $|B|=0.5 \mathrm{~T}$. The largest history-dependent difference is evident for $B=0.2 \mathrm{~T}$, when $\mathrm{M}_{I P}$ is along $\mathrm{X}+$ or $\mathrm{Y}+$, and for $B=-0.2 \mathrm{~T}$ when $\mathrm{M}_{I P}$ is along $\mathrm{X}$ - or $\mathrm{Y}$-. Analogous to fig. 5.5(d), the large-contrast oscillations occur about very different mean values, and are somewhat phase shifted. At $B=0 \mathrm{~T}$, when $\mathrm{M}$ lies along $\mathrm{X}+(0) / \mathrm{X}-(0)$, the $\mathrm{M}$ dynamics shows only a small history dependence. The "anomalous" jumps in the coherent magnetization dynamics 


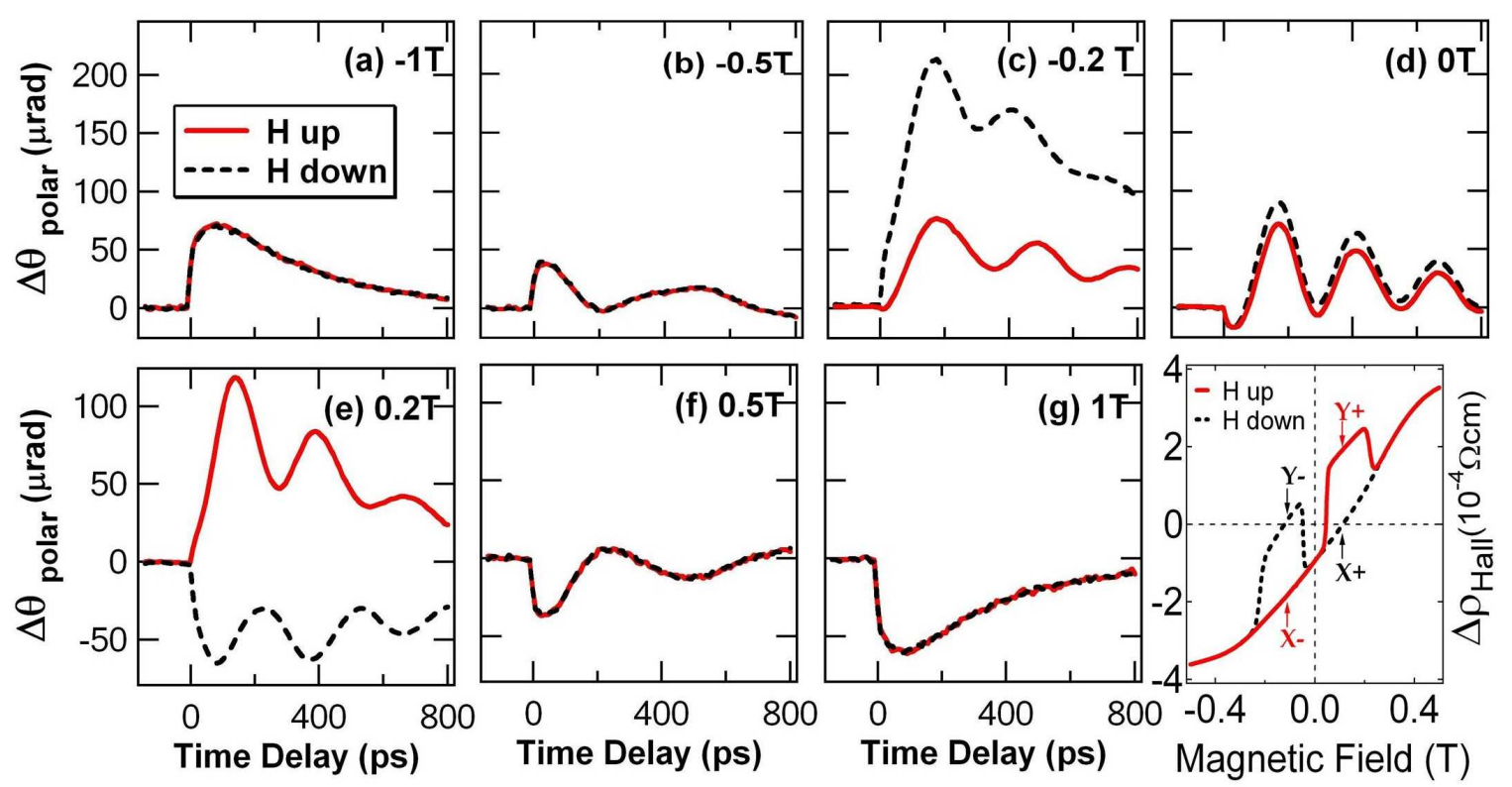

Figure 5.6: Magnetic memory shows hysteresis effects in the coherent magnetization dynamics.

correspond to $\mathrm{M}_{I P}$ jumps from $\mathrm{X}+$ to $\mathrm{Y}+$ to $\mathrm{X}+$ (i.e., on the up sweep, from $0 \mathrm{~T}$ to $0.2 \mathrm{~T}$ to $0.5 \mathrm{~T}$ ), and to $\mathrm{M}_{I P}$ jumps from $\mathrm{X}$ - to $\mathrm{Y}$ - to $\mathrm{X}$ - (i.e., on the down sweep, from $0 \mathrm{~T}$ to $-0.2 \mathrm{~T}$ to $-0.5 \mathrm{~T})$, respectively.

These results, together with our magnetic field scans of $\Delta \theta_{K}$ on a ps timescale presented in the previous section (Fig. 5.2(a)), support the picture of non-thermal effects (i.e., $\left.\Delta B_{c}\right)$ initiating the magnetization dynamics. As seen in the zero-field magnetic memory states, the difference in the initiation of coherent magnetization dynamics between states with $\mathrm{M}_{I P}$ components aligned along different directions turns out to have long-lasting effects persisting at the ns-timescale. 


\subsubsection{Thermal and non-thermal effects in coherent magnetization dynamics}

In order to further elucidate the coherent magnetization dynamics at the ns timescale, we need to understand the dependence of the magnetic anisotropy fields on temperature. To shed insight into temperature effects on easy axes directions, we follow the variation with temperature of the M polar component dynamics, when $\mathrm{M}$ is initially prepared in the $\mathrm{X}+(0)$ state.

Figure 5.7 shows the polar component $\Delta \theta_{K}(\mathrm{t})$ of magnetization dynamics for temperatures as low as $5 \mathrm{~K}$, and as high as $90 \mathrm{~K}$, above $\mathrm{T}_{C}$ of $77 \mathrm{~K}$. For traces below $30 \mathrm{~K}$, the magnetization is initially kicked off by the magnetic field pulse $\Delta B_{c}$ to a negative $\hat{z}$-axis direction, after which it begins to precess. This precession takes place with a frequency and amplitude decreasing with temperature. At $35 \mathrm{~K}$ the oscillations completely disappear. Above $35 \mathrm{~K}$, we only observe a small M reorientation, which gradually disappears when approaching $\mathrm{T}_{C}$.

The behavior observed below $30 \mathrm{~K}$, when the easy axis is [100], is well described by the mechanism we presented above. The M is initially kicked off by the short-lived, ps magnetic field pulse $\Delta B_{c}$. Following transfer of energy from the photoexcited hot hole population to the lattice, the easy axis direction changes from [100] (at low temperatures, as shown in fig. 5.7 inset) towards [11̄0]. Consequently, $\mathrm{M}$ begins precession around this slowly varying easy axis direction. Qualitatively, at higher temperatures the amplitude of the magnetic field pulse induced by the hot holes is lower, as is the strength of the anisotropy field in general. As the temperature increases, the initial easy axis direction changes from 


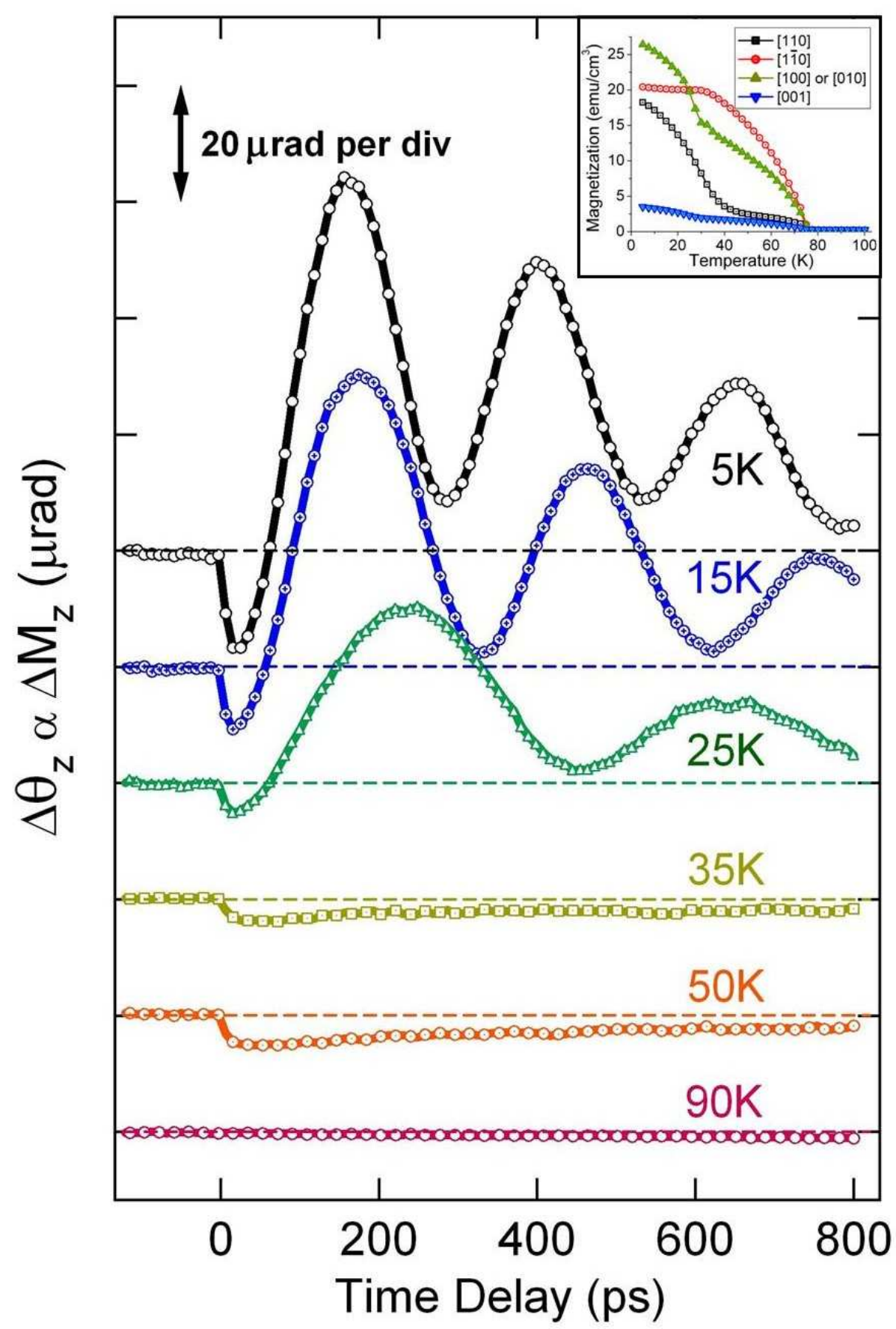

Figure 5.7: Temperature dependence of $\hat{z}$ axis projection of magnon precession. Oscillations are visible up to $\sim 30 \mathrm{~K}$, after which they disappear due to the change in easy axis direction from [100] to [110] (shown in the inset). 
purely along [100], to between [100] and [110]. This explains both the decreased $\Delta \theta_{K}(\mathrm{t})$ initial displacement, and the lower amplitude and frequency of the precession observed. Around $30 \mathrm{~K}$, an easy axis transition takes place from [100] type to [110] type, as shown in the figure 5.7 inset. The new [110] direction persists until the temperatures reach $\mathrm{T}_{C}$. Therefore, above $30 \mathrm{~K}, \mathrm{M}$ lies initially along [11̄0]. Consequently, a magnetic field pulse or stronger anisotropy field along this direction will not initiate precession. The only effect seen is a much smaller reorientation of $\mathrm{M}$ along the $\hat{z}$-axis direction, which can be explained by a small transient magnetic field perpendicular to the sample induced by the long-lived holes. As previously discussed in $[98,66]$ this easy axis transition takes place at a temperature of the order of about half of $\mathrm{T}_{C}$.

We phenomenologically fit the magnetization precession we observe along the $\hat{z}^{-}$ axis with an equation including a damped precessional term, and a damped non-oscillatory term. An example of the fit is presented in figure 5.8:

$$
\Delta \theta_{K}=A e^{-t / \tau_{1}} \cos (\omega t+\phi)+B\left(1-e^{-t / \tau_{2}}\right) e^{-t / \tau_{3}}
$$

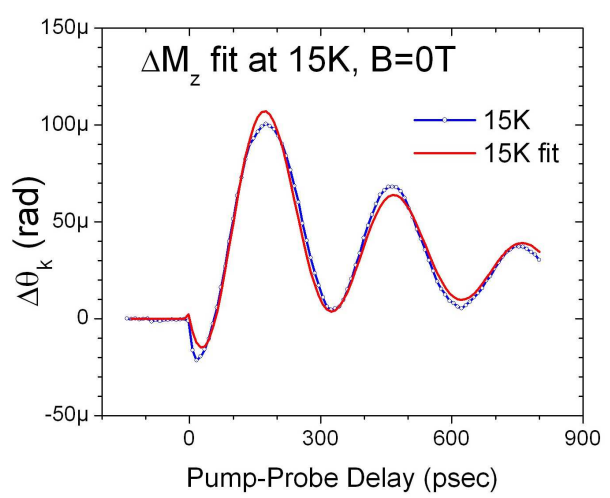

Figure 5.8: Phenomenological fit of coherent magnetization dynamics includes a damped precessional term, with a time constant $\tau_{1}$, as well as a non-oscillatory term that takes $\tau_{2}$ to build up, and decays on $\tau_{3}$ timescale. 
In figure 5.9, we show the temperature dependence of precession frequency $\omega$. As we can clearly see in the dynamic traces of the magnetization along the $\hat{z}$-axis, the oscillations slow down with increasing temperature, as a sign of weakening magnetic anisotropy fields [52] (Fig. 5.10). Above $30 \mathrm{~K}$, the oscillations are quickly quenched, due to the reorientation of the easy axis along the [110] direction, as discussed above.

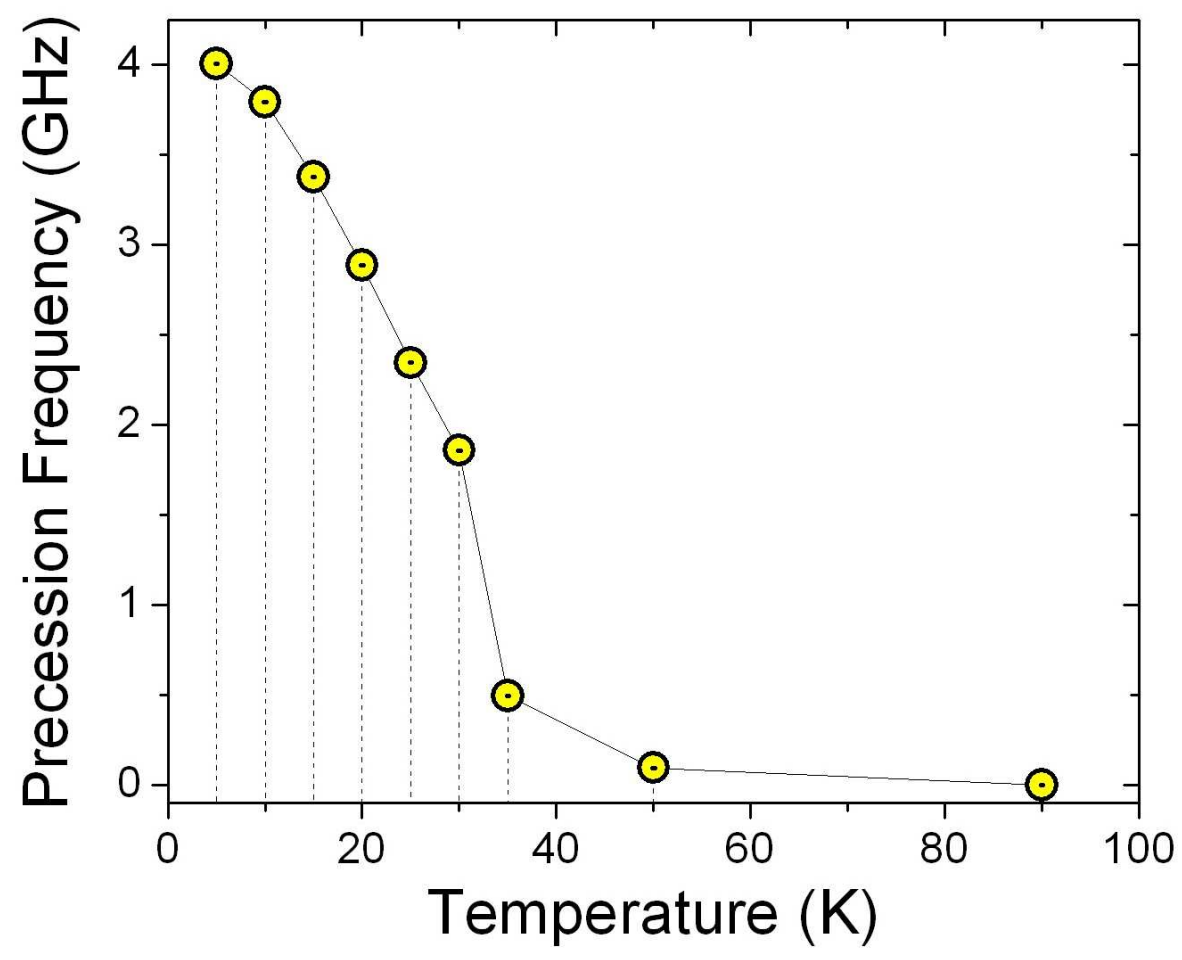

Figure 5.9: Magnetization precession frequency $\nu=\omega /(2 \pi)$ decreases with increasing temperature in sample A due to weakening magnetic anisotropy fields. Above $30 \mathrm{~K}$, a transition in the easy axis direction takes place from [100] to [110], which quickly quenches all oscillations.

We thus confirm the expected ferromagnetic resonance due to both thermal and non-thermal effects, manifesting itself as initial $\mathrm{M}$ reorientation due to a hot-carrier induced magnetic field pulse, precession around an effective magnetocrystalline anisotropy field, and easy axis reorientation. 


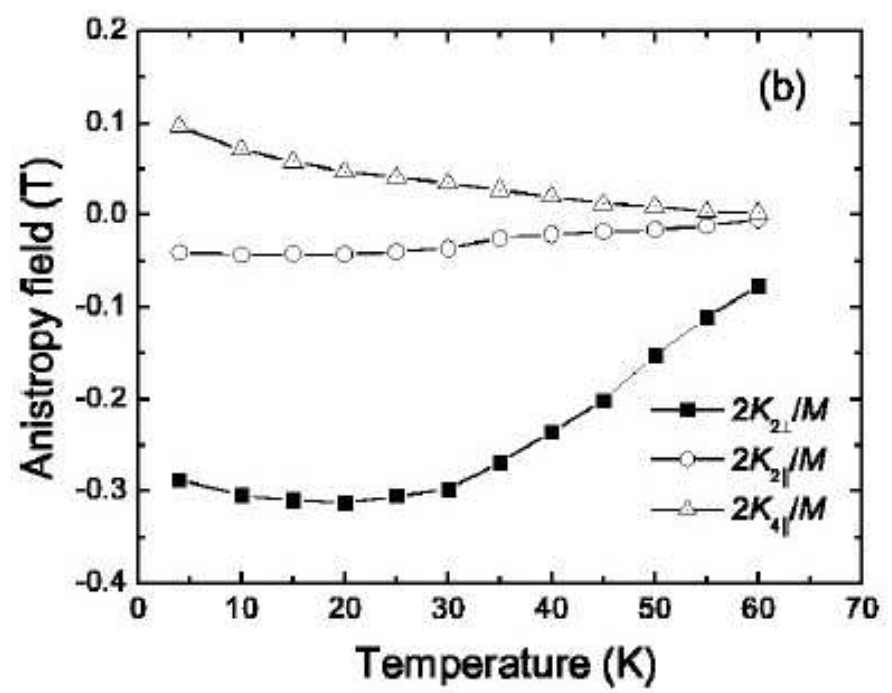

Figure 5.10: From ref. [52]: Magnetic anisotropy fields $2 \mathrm{~K}_{2 \perp} / \mathrm{M}, 2 \mathrm{~K}_{2 \|} / \mathrm{M}$, and $2 \mathrm{~K}_{4 \|} / \mathrm{M}$ as a function of temperature for a $\mathrm{Ga}_{0.97} \mathrm{Mn}_{0.03} \mathrm{As} / \mathrm{GaAs}$ sample. The solid lines are guides for the eye.

In general, the precessional behavior is due to a photoexcited FM resonance, or magnon, which represents the fundamental quantum of the collective magnetization motion. The magnetization dynamics is well described by the Landau-Lifshitz-Gilbert equation of motion $[99,26]$ :

$$
\frac{d \mathbf{M}}{d t}=-\gamma\left[\mathbf{M} \times\left(H+H_{e f f}\right)\right]+\frac{G}{\gamma M^{2}} \mathbf{M} \times \frac{d \mathbf{M}}{d t}
$$

where $\mathrm{H}$ is the externally applied DC magnetic field $\mathrm{H}, \mathrm{H}_{\text {eff }}$ is the effective magnetic field including the magnetic field pulse, demagnetizing field and magnetocrystalline anisotropy fields, $\gamma=g \mu_{B} \hbar^{-1}$ is the gyromagnetic ratio (g: spectroscopic splitting factor), and $\mathrm{G}$ the Gilbert damping parameter. The precession frequency is determined from the magnetic free energy density, which describes the easy axis of magnetization [52]:

$$
\begin{array}{r}
F=-M H\left[\cos \theta \cos \theta_{H}+\sin \theta \sin \theta_{H} \cos \left(\phi-\phi_{H}\right)\right]-2 \pi M^{2} \sin ^{2} \theta-K_{2 \perp} \cos ^{2} \theta \\
\ldots-\frac{1}{2} K_{4 \perp} \cos ^{4} \theta-\frac{1}{2} K_{4 \|} \frac{1}{4}(3+\cos 4 \phi) \sin ^{4} \theta-K_{2 \|} \sin ^{2} \theta \sin ^{2}\left(\phi-\frac{\pi}{4}\right)
\end{array}
$$


where the first term describes the Zeeman energy, the second term is the demagnetizing energy (shape anisotropy), $\mathrm{K}_{2 \perp}$ and $\mathrm{K}_{4 \perp}$ are constants representing the perpendicular uniaxial and cubic anisotropy, respectively, while $\mathrm{K}_{2 \|}$ and $\mathrm{K}_{4 \|}$ are the planar uniaxial and cubic anisotropy, respectively. The angles $\theta$ and $\phi$ are polar coordinates with respect to the $\hat{z}$-axis (perpendicular to the sample plane) and the $\hat{x}$-axis (along [100]). The in-plane anisotropy term $K_{2 \|}$ reflects the fact that in a zinc blende structure the [110] and [1ํㅣ directions are not equivalent. The ferromagnetic resonance frequency is then given by the double derivatives $\mathrm{F}_{\theta \theta}, \mathrm{F}_{\phi \phi}$, and $\mathrm{F}_{\theta \phi}$ of the anisotropic part of magnetic free energy density F [26]:

$$
\omega=\frac{\gamma}{M \sin \theta} \sqrt{F_{\theta \theta} F_{\phi \phi}+F_{\theta \phi}^{2}}
$$

The magnon frequency in the low field limit is well described analytically by the formula:

$$
\omega_{m a g}=\gamma \sqrt{H_{4 \|}\left(H_{4 \|}+H_{2 \perp}\right)}
$$

where $H_{2 \perp}$ and $H_{4 \|}$ are defined in terms of anisotropy energies $\mathrm{Ki}$ as $4 \pi M-2 K_{2} / M$ and $2 K_{4 \|} / M$, representing the effective uniaxial and cubic anisotropy fields, respectively. We estimate $H_{2 \perp}$ and $H_{4 \|}$ to be $-0.3 \mathrm{~T}$ and $0.06 \mathrm{~T}$ at $5 \mathrm{~K}$ in our sample, from the experimentally measured hard axis magnetization curve. This yields a magnon frequency $\omega_{m a g}=4.12 \mathrm{GHz}$, in close agreement with the measured value (Fig. 5.11).

However, at high external magnetic fields, damping of the magnetization precession is considerable, quenching all precession within much less than a single cycle. In figure 5.12 , we follow the field dependence of the magnetization precession at a low temperature, $\mathrm{T}=5 \mathrm{~K}$, along an entire hysteresis loop between $\pm 1 \mathrm{~T}$. The magnetization trajectories and frequencies are independent of magnetic field history, except for $|B|=0.1 \mathrm{~T}$ and $0.2 \mathrm{~T}$, 


\section{Frequency $(\mathrm{GHz})$}

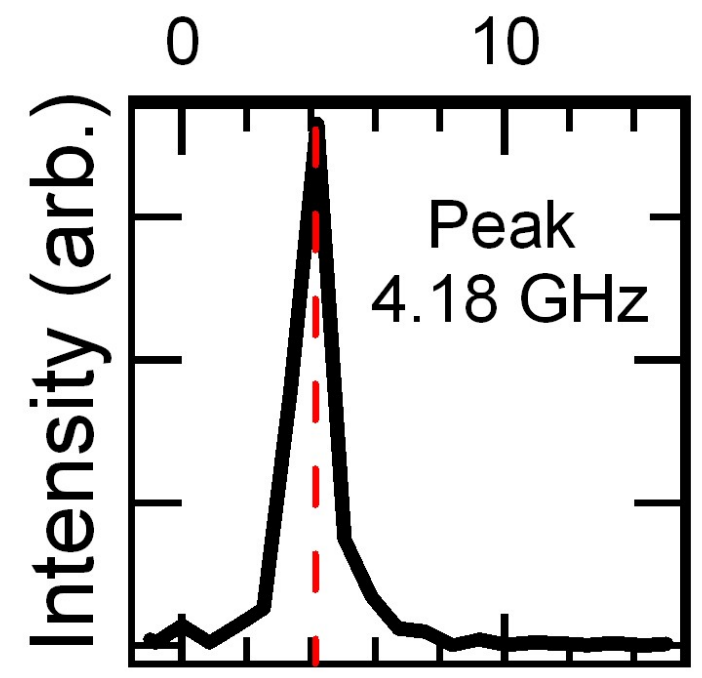

Figure 5.11: The Fourier transform of the coherent magnetization oscillation at $\mathrm{B}=0 \mathrm{~T}$ and $\mathrm{T}=5 \mathrm{~K}$ yields a magnon frequency of $4.18 \mathrm{GHz}$, in close agreement with the calculated value.
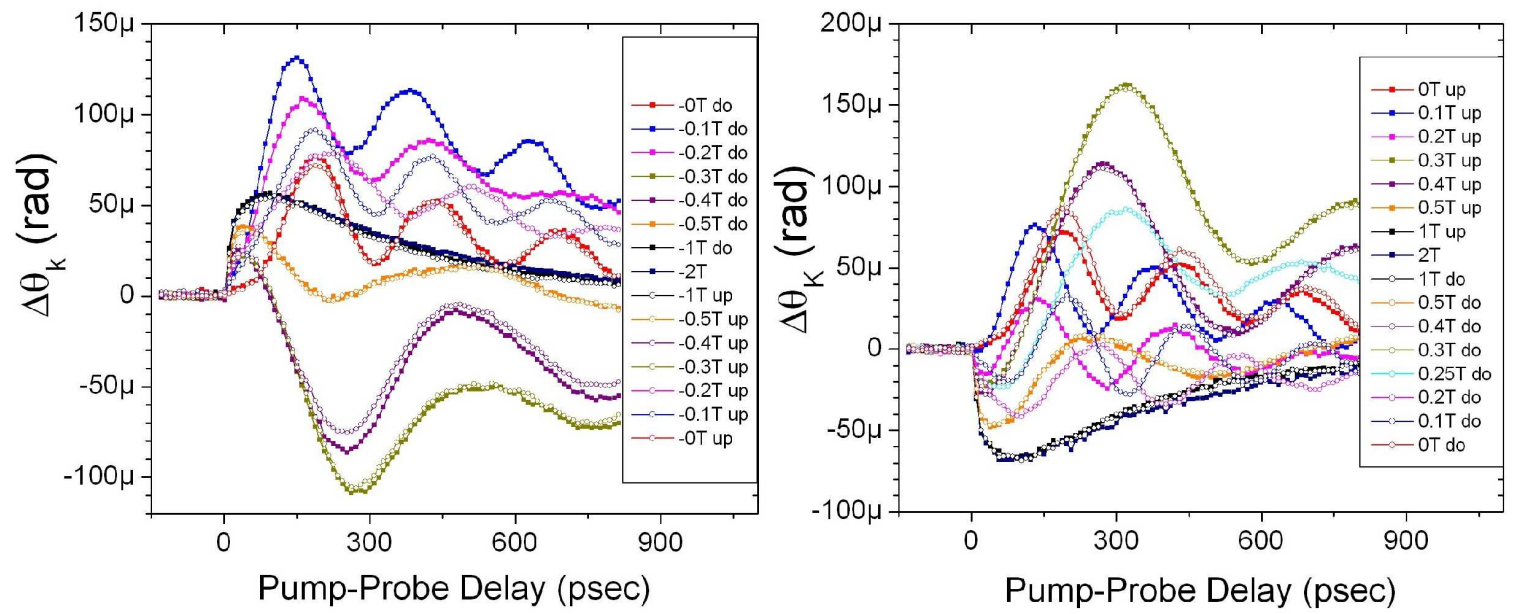

Figure 5.12: Magnetic field dependence of magnetization dynamics at a low temperature $\mathrm{T}=5 \mathrm{~K}$. Left and right panels present $\mathrm{M}$ dynamic traces taken along the negative/ positive field hysteresis loop $(-2 \mathrm{~T}<B<2 \mathrm{~T})$; up/do = up/down sweep. The traces are fairly identical when $\mathrm{M}_{I P}$ is aligned along the same direction, independent of $\mathrm{B}$ field history, but they differ when $\mathrm{M}_{I P}$ is aligned along different, $\mathrm{X} \pm / \mathrm{Y} \pm$ directions. 
when they display memory effects.

We extract the frequency of the oscillations observed as a function of external magnetic field applied using eqn. 5.2 in figure 5.13. If we compare this frequency to the rough estimate of magnon frequency obtained using eqn. 5.6, in which $H_{2 \perp}$ is replaced by $H_{2 \perp}+H_{e x t}$, we find that qualitatively, the results are very similar. At low magnetic fields, the magnon frequency initially decreases as a function of $H_{\text {ext }}$ because $H_{2 \perp}$ is negative. Therefore, we only observe an increase in $\omega_{\text {mag }}$ above $0.3 \mathrm{~T}$, when $H_{\text {ext }}$ overcomes $H_{2 \perp}$. At large magnetic fields (i.e., $H_{\text {ext }}>0.5 \mathrm{~T}$ ), the oscillations are completely quenched. As the external magnetic field applied increases, it forces the magnetization to align almost perfectly along it. Therefore, when the pump pulse hits the sample, it is unable to significantly tilt $\mathrm{M}$ away from this position. Thus, with extremely small initial M reorientations, oscillations are not observed.

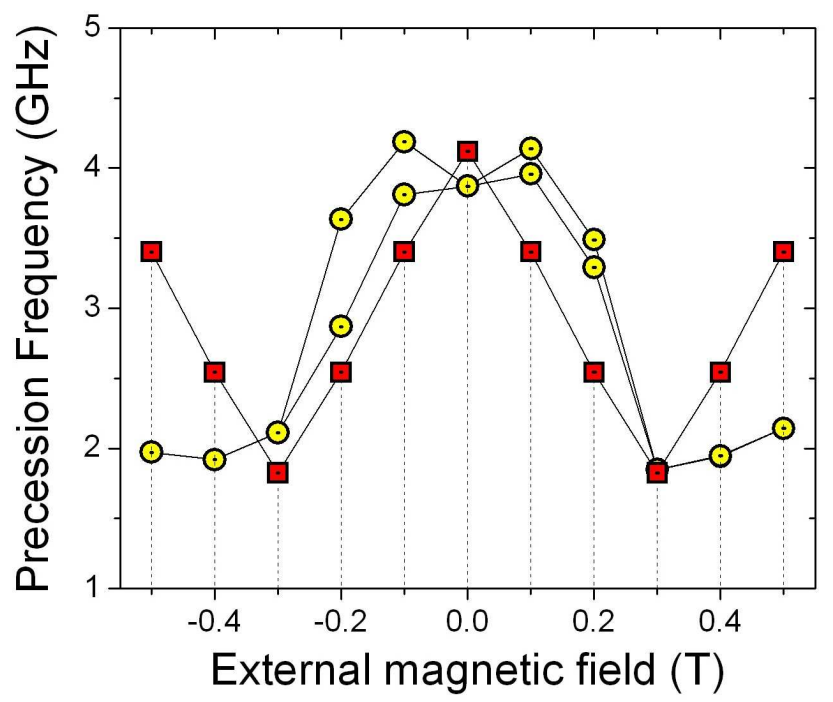

Figure 5.13: As expected, the magnetic field dependence of precession frequency indicates that the magnon frequency decreases with increasing external field for $\left|H_{e x t}\right|=0-0.3 \mathrm{~T}$, from $\sim 4 \mathrm{GHz}$ to $\sim 2 \mathrm{GHz}$. Above $0.3 \mathrm{~T}$, the magnon frequency begins to increase. At high magnetic fields, oscillations cannot be observed due to a very small initial $\mathrm{M}$ tilt. 


\subsection{Conclusion}

We have investigated the coherent magnetization dynamics in (Ga,Mn)As both at the sub-ps and at the ns timescale, and have uncovered both thermal and non-thermal effects responsible for the behavior observed.

At the sub-ps timescale, we observed an ultrafast reorientation of the magnetization vector at UV photo-excitation, sensitive to the orientation of the in-plane magnetization component. We identified a non-thermal, carrier-mediated mechanism responsible for this fs magnetization rotation, and employed it to design an ultrafast scheme for detection of magnetic memory. Magnetic field scans obtained using this method show hysteresis behavior at the fs timescale, which allowed us to unequivocally detect four state magnetic memory with sub-ps speed.

At the ns timescale, we observed coherent magnetization dynamics driven by both non-thermal and thermal effects. The non-thermal effects are responsible for the initial displacement of the magnetization vector, and cease within the first 1 ps. On longer timescales, the UV pump pulse energy transfers to the lattice and changes the magnetic anisotropy fields, initiating precession of the magnetization. This photo-excited FM resonance, or magnon, carries along signatures of the non-thermal effects, allowing to differentiate mag-

netic memory states also at the ns timescale. We identified a change in easy axis direction in ns coherent magnetization dynamics around $\mathrm{T}=30 \mathrm{~K} \sim \mathrm{T}_{C} / 2$, and confirmed the magnon frequency magnetic field dependence expected at low temperatures and low external magnetic fields. 


\section{Chapter 6}

\section{Conclusion}

We studied ultrafast magneto-optical processes in ferromagnetic (Ga,Mn)As induced by UV femtosecond laser pulses. We focused our attention on two main challenges facing spintronics applications: ultrafast, technologically relevant optical control of $\mathrm{T}_{C}$ and magnetic memory detection.

We have made the first observation of photo-enhanced ferromagnetism in dilute magnetic semiconductors on ultrafast timescales. Our data taken on a LT-MBE-grown (Ga,Mn)As sample clearly shows that a dynamic magnetization buildup occurs on a 100 ps timescale, associated with a transient $\sim 1 \mathrm{~K}$ increase of $\mathrm{T}_{C}$. Our analysis and theoretical simulations explain the salient features of the experimental observations, demonstrating in particular a correlation-induced peak below $\mathrm{T}_{C}$ around $20 \mathrm{~K}$. We have observed transient signatures of an ultrafast photoinduced $\mathrm{PM} \rightarrow \mathrm{FM}$ phase transition above $\mathrm{T}_{C}$, resulting from the transient strengthening of the Mn-hole correlation via photoexcited holes. We found that at ultrafast timescales, a small but finite magnetic field is needed to ensure single 
domain growth, in order to observe the magnetic phase transition. To further our understanding, we expanded our study to include quasi-2D LT-MBE (Ga,Mn)As. In search for a considerably stronger enhancement of FM, we surprisingly found an unexpected transition at a temperature considerably below $\mathrm{T}_{C}$, evident only in the dynamical magneto-optical properties of the system, but not in the static measurements. We tentatively attributed this transition to a half-metal to metal transition, underlying the very interesting dynamics aspects of the charge-spin interplay.

Another novel observation is the giant ultrafast photoenhancement of ferromagnetism in II-PLM GaMnAs. At temperatures below $20 \mathrm{~K}$, we found that the magnitude of the dynamic magnetization enhancement is on the order of $10 \%$ of the static value, much larger than in the LT-MBE samples. We interpreted the observed giant photoenhanced ferromagnetism to be due to a PM $\rightarrow$ FM phase transition occurring in the top layers of the sample. However, the amplitude of the dynamic magnetization enhancement is quickly quenched and disappears above $20 \mathrm{~K}$. The subtle nature of magnetization enhancement, highly sensitive to the uneven $\mathrm{Mn}$ and hole distributions in this sample, is responsible for the quenching of this phenomenon above $20 \mathrm{~K}$. We confirmed in a compensated sample that selectively decreasing the hole concentration in the regions of low local $\mathrm{T}_{C}$ (that would normally undergo phase transition) effectively kills the giant enhancement of ferromagnetism.

Switching themes, we showed for the first time that the rich magnetic memory effects in (Ga,Mn)As manifest themselves not only in static measurements, but also at the ultrafast timescales, and can be utilized for magnetic memory detection with sub-ps speed. We have investigated the coherent magnetization dynamics in $(\mathrm{Ga}, \mathrm{Mn})$ As at the sub-ps and 
ns timescales, and have uncovered both thermal and non-thermal effects responsible for the behavior observed. At the sub-ps timescale, we have observed an ultrafast reorientation of the magnetization vector following UV photo-excitation, sensitive to the orientation of the in-plane magnetization component. Based on this effect, we designed an ultrafast magnetic memory detection scheme. We used this scheme to observe hysteretic magnetic field scans at the fs timescale, which allowed us to unequivocally detect four state magnetic memory with sub-ps speed in $(\mathrm{Ga}, \mathrm{Mn})$ As. We identified that a non-thermal, carriermediated mechanism, manifesting itself as a magnetic field pulse, is responsible for the fs magnetization rotation observed. This mechanism may represent an universal principle in all carrier-mediated ferromagnetic materials, and is especially promising for future applications using, for example, oxides (which hold promise of far-above room temperature $\mathrm{T}_{C}$ ). At the ns timescale, we observed coherent magnetization dynamics driven by both non-thermal and thermal effects.

Our measurements reveal new fundamental transient collective magnetic processes on ultrafast time scales in (Ga,Mn)As and identify the critical role of the non-thermal Mnhole exchange correlation in photoinduced phenomena. The new functionalities in the ps and sub-ps timescales reported here provide exciting prospects for studying competing phases in strongly correlated magnetic materials and may open future opportunities for high-speed spin-photon-charge integrated devices. 


\section{Appendix A}

\section{MOKE: Probe Polarization}

\section{Rotation vs. Ellipticity Change}

We give an example of the MOKE-MCD signal overlap in sample A, showing that the transient signal changes are of magnetic origin. The normalized signals show an almost perfect overlap (especially in the short, ps-timescale). According to eqns. 2.14, 2.16, and 2.20 , this means that either the complex proportionality prefactor $\tilde{F}$ is not time dependent, or if it is, its transient relative change over the course of $1 \mathrm{~ns}$ is much smaller than the relative magnetization changes (see Eqn. 2.22). 


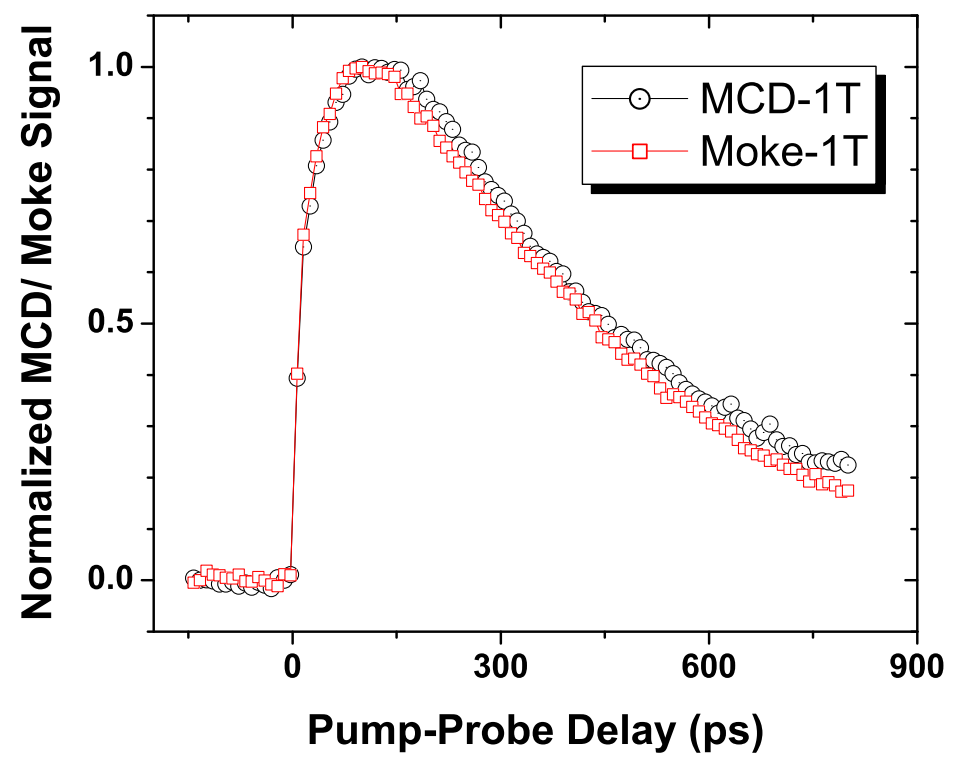

Figure A.1: Overlap of the MOKE-MCD signals on a "long" timescale up to 900 ps.

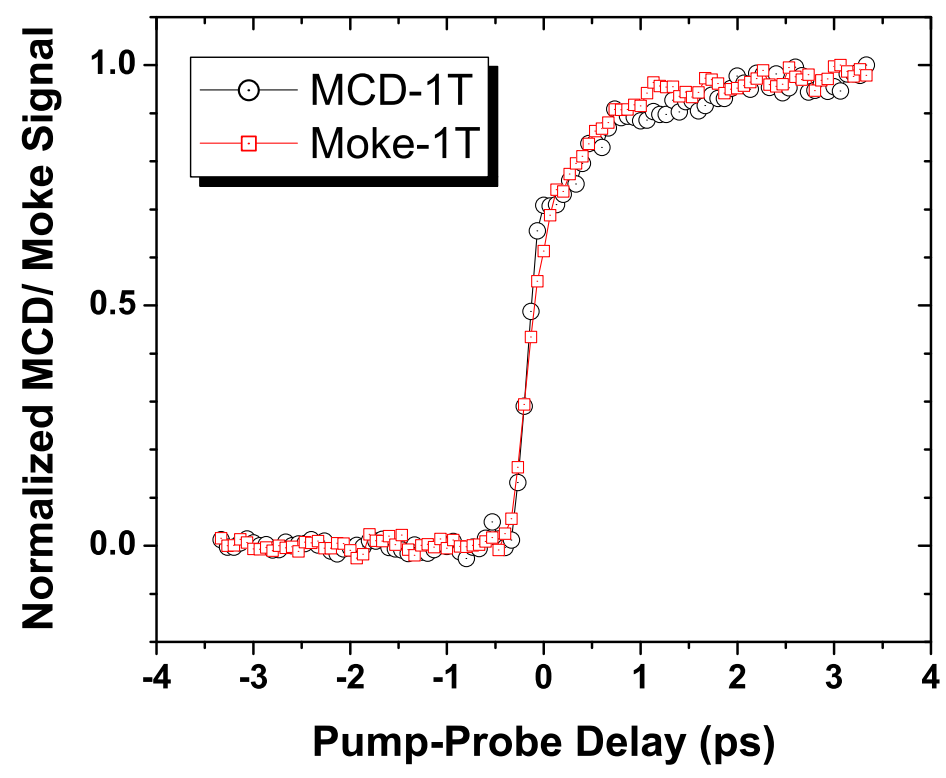

Figure A.2: Overlap of the MOKE-MCD signals on the "short", ps timescale. 


\section{Appendix B}

\section{Data Acquisition}

To accurately collect transient data, the lock-in amplifier, linear stage controller, and rotational stage controller are connected to a PC via GPIB and connectors. Various electronics can be thus controlled remotely from a central computer. The data collection is substantially simplified by using LabVIEW (by National Instruments, Inc.), a visual programming language commonly used for data acquisition and instrument control.

The rotation stage position (and thus probe beam polarization) is controlled via vendor supplied software, Sigma Koki SG Commander. Used in conjunction with an inhouse LabVIEW code, "testdetector.vi" (Fig. B.1), it allows for balancing the MOKE/MCD detector. Testdetector.vi reads in the lock-in amplifier signal from the balanced detector at specified time-intervals (in $100 \mathrm{~ms}$ units). To balance the detector, the rotation stage is moved until testdetector.vi reads, on average, a 0 signal. Another in-house LabVIEW code, "StageMove.vi", is used to control the linear stage position (Fig. B.2) to enable precision positioning of the translation stage for specific pump-probe time delays. 


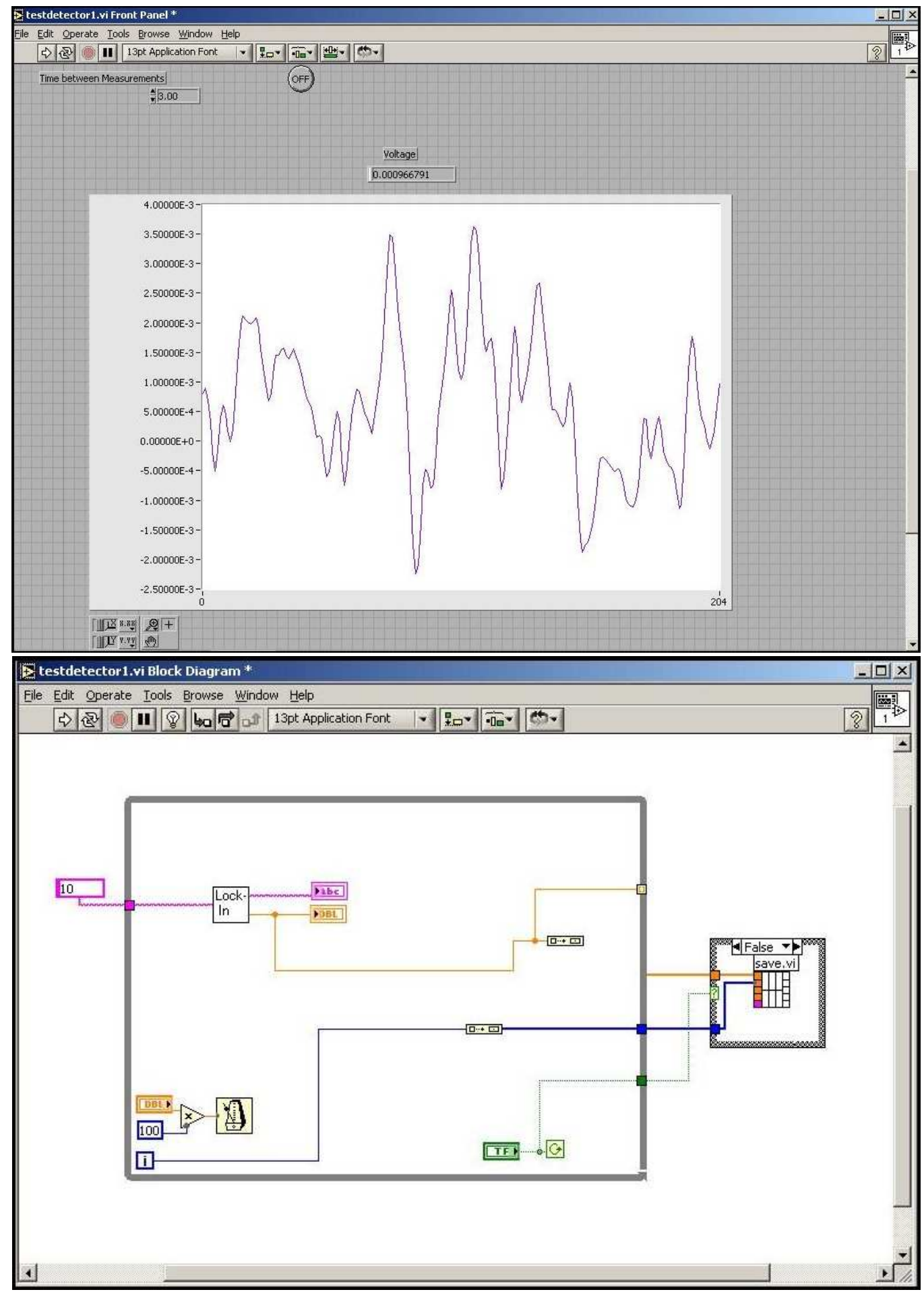

Figure B.1: Testdetector.vi user interface, with LabVIEW block diagram depicted below. 


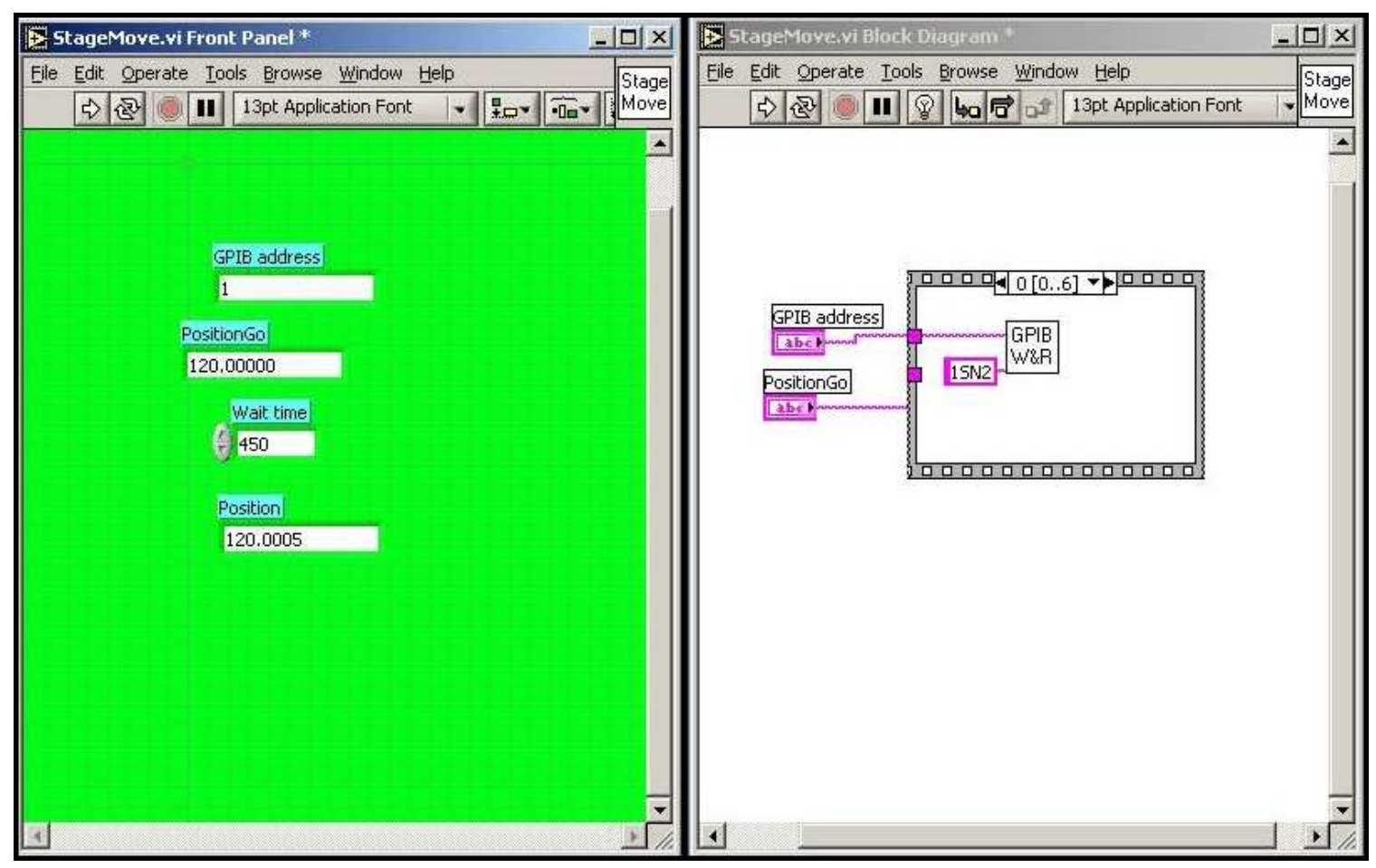

Figure B.2: Stagemove.vi user interface and LabVIEW block diagram.

Perhaps the most useful LabVIEW code, "PP.vi" (i.e., pump-probe), allows us to record transient signals (MOKE, MCD, $\Delta \mathrm{R} / \mathrm{R}$ ). The user can specify the measured time range, interval time-step, and the total number of points to be recorded. Moreover, the user can set a particular integration time for the lock-in amplifier; PP.vi will wait for a time usually three times as long before reading the value from by the lock-in (Fig. B.3). The output files are saved in a format that is imported into Origin or other scientific graphing and data analysis software. 


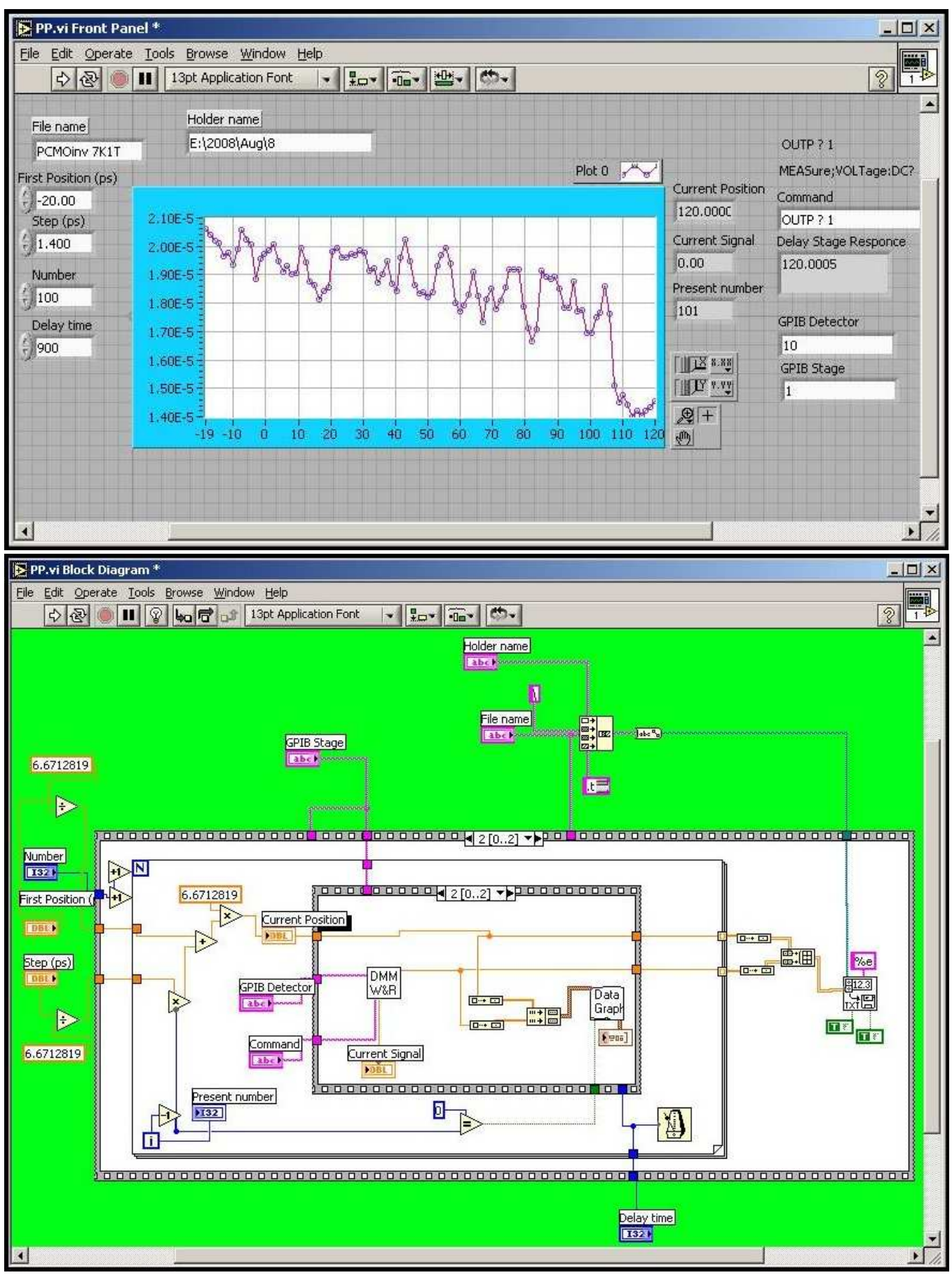

Figure B.3: PP.vi user interface with LabVIEW block diagram depicted below. 


\section{Appendix $\mathrm{C}$}

\section{Magnetization Dynamics in 3D}

At $\mathrm{B}=0 \mathrm{~T}$, the $3 \mathrm{D}$ trajectory of the magnetization vector can be visualized by a combination of polar and transverse MOKE measurements:

- polar MOKE yields $\Delta \theta_{z}(t) \propto \Delta M_{z}(t)$.

- transverse MOKE (i.e., in the $\Delta \mathrm{R} / \mathrm{R}$ setup, but below $\mathrm{T}_{C}$ ), with incident light $\mathrm{p}$ polarized $\left(\operatorname{tMOKE}(\hat{p})\right.$, yields $\Delta M_{x}(t)$ :

$$
\Delta \theta_{x}(t)=\frac{1}{2 R_{x}}[t M O K E(\hat{p}, 0 T)-t M O K E(\hat{p}, 2 T)] \propto \Delta M_{x}(t)
$$

We subtract the $2 \mathrm{~T} \operatorname{tMOKE}(\hat{p})$ signal from the $0 \mathrm{~T}$ signal to eliminate the nonmagnetic contributions to $\operatorname{tMOKE}(\hat{p}, 0 \mathrm{~T})$, since we know that at $2 \mathrm{~T}$, the magnetization is saturated along $\hat{z}$, i.e. there is no $M_{x}$ component.

- a combination of transverse MOKE signal with incident light polarized at $45^{\circ} \hat{s}$ (i.e., $\hat{s}+\hat{p})$ and $45^{\circ} \hat{p}$ (i.e., $\hat{s}-\hat{p}$ ), gives $\Delta M_{y}(t)$ :

$$
\Delta \theta_{y}(t)=\frac{1}{2 R_{y}}\left[\operatorname{MOK} E\left(45^{o} \hat{s}\right)-t \operatorname{MOKE}\left(45^{o} \hat{p}\right)\right] \propto \Delta M_{y}(t)
$$


The $t M O K E\left(45^{\circ} \hat{s}\right)$ signal is proportional to $I_{45 s}=R_{0}+R_{x} M_{x}+R_{y} M_{y}$ while the $t M O K E\left(45^{\circ} \hat{p}\right)$ signal is proportional to $I_{45 p}=R_{0}+R_{x} M_{x}-R_{y} M_{y}$, hence

$$
M_{y}=\frac{I_{45 s}-I_{45 p}}{2 R_{y}}
$$

and the transient $\Delta M_{y}(t)$ is given by the transient two intensities.

This technique is relevant mostly at $0 \mathrm{~T}$, since even at small fields, the magnetization gets tilted perpendicular to the sample plane, along the hard axis. One such example of a magnetization trajectory is illustrated in figure C.1 and shows a magnon precession.

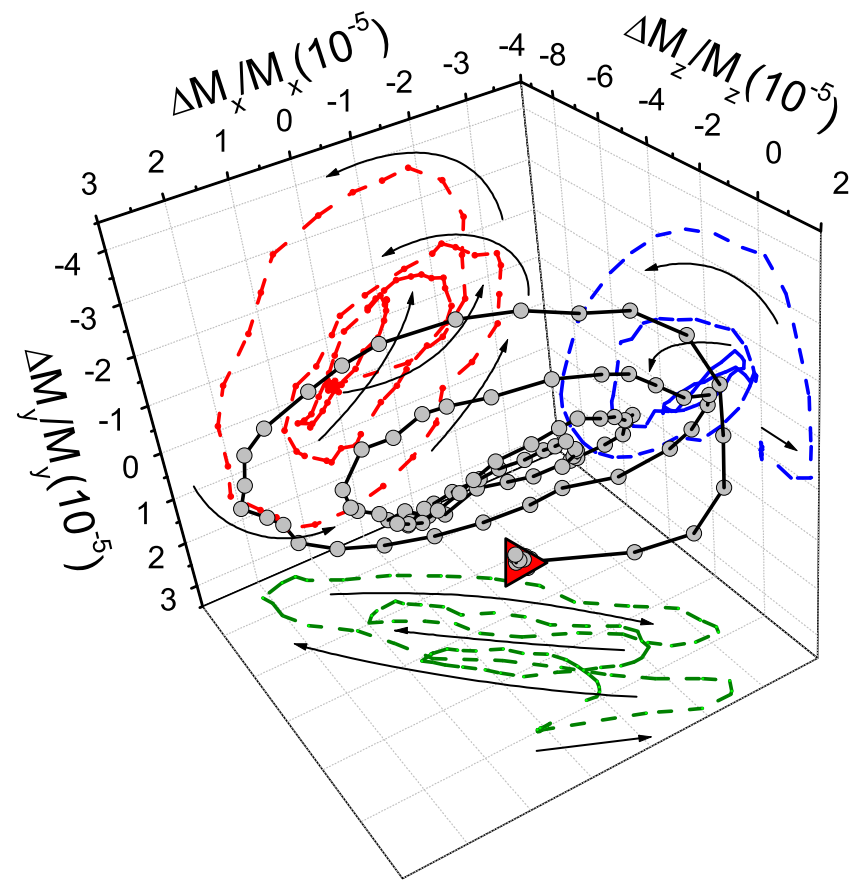

Figure C.1: Visualizing magnon precession by following the 3D magnetization vector dynamics. The main curve (grey dots) shows the pump-induced trajectory of the magnetization vector (at $\mathrm{B}=0 \mathrm{~T}$ ) on a $900 \mathrm{ps}$ timescale. Dashed lines are the $2 \mathrm{D}$ projections. 


\section{Bibliography}

[1] H. Åsklund, L. Ilver, J. Kanski, J. Sadowski, and R. Mathieu. "Photoemission studies of $\mathrm{Ga}_{1-x} \mathrm{Mn}_{x}$ As: Mn concentration dependent properties" Phys. Rev. B, 66, 115319 (2002).

[2] H. Akinaga and H. Ohno. "Semiconductor Spintronics" IEEE Transactions on nanotechnology, 1(19), 2002.

[3] D. D. Awschalom, M. R. Freeman, N. Samarth, H. Luo, and J. K. Furdyna. "Observation of polaron dynamics in magnetic quantum wells" Phys. Rev. Lett., 66, 1212 (1991).

[4] David D. Awschalom and Michael E. Flatté. "Challenges for semiconductor spintronics" Nature Phys., 3, 153 (2007).

[5] M. N. Baibich, J. M. Broto, A. Fert, F. Nguyen Van Dau, F. Petroff, P. Etienne, G. Creuzet, A. Friederich, and J. Chazelas. "Giant Magnetoresistance of (001)Fe/(001)Cr Magnetic Superlattices" Phys. Rev. Lett., 61, 2472 (1988).

[6] T. Baron, S. Tatarenko, K. Saminadayar, N. Magnea, and J. Fontenille. "Plasma 
nitrogen doping of $\mathrm{ZnTe}, \mathrm{Cd}_{1-x} \mathrm{Zn}_{x} \mathrm{Te}$, and CdTe by molecular beam epitaxy" Appl. Phys. Lett., 65, 1284 (1994).

[7] E. Beaurepaire, J.-C. Merle, A. Daunois, and J.-Y. Bigot. "Ultrafast Spin Dynamics in Ferromagnetic Nickel" Phys. Rev. Lett., 76, 4250 (1996).

[8] L. Bergqvist, P. A. Korzhavyi, B. Sanyal, S. Mirbt, I. A. Abrikosov, L. Nordström, E. A. Smirnova, P. Mohn, P. Svedlindh, and O. Eriksson. "Magnetic and electronic structure of $\mathrm{Ga}_{1-x} \mathrm{Mn}_{x} \mathrm{As} "$ Phys. Rev. B, 67, 205201 (2003).

[9] J.-Y. Bigot, L. Guidoni, E. Beaurepaire, and P. N. Saeta. "Femtosecond Spectrotemporal Magneto-optics" Phys. Rev. Lett., 93, 077401 (2004).

[10] G. Binasch, P. Grünberg, F. Saurenbach, and W. Zinn. "Enhanced magnetoresistance in layered magnetic structures with antiferromagnetic interlayer exchange" Phys. Rev. B, 39, 4828 (1989).

[11] J. Blinowski and P. Kacman. "Spin interactions of interstitial Mn ions in ferromagnetic GaMnAs" Phys. Rev. B, 67, 121204 (2003).

[12] K. S. Burch, J. Stephens, R. K. Kawakami, D. D. Awschalom, and D. N. Basov. "Ellipsometric study of the electronic structure of $\mathrm{Ga}_{1-x} \mathrm{Mn}_{x} \mathrm{As}$ and low-temperature GaAs" Phys. Rev. B, 70, 205208 (2004).

[13] R. E. Camley and J. Barnaś. "Theory of giant magnetoresistance effects in magnetic layered structures with antiferromagnetic coupling" Phys. Rev. Lett., 63, 664 (1989).

[14] H. C. Casey Jr., D. D. Sell, and K. W. Wecht. "Concentration dependence of the 
absorption coefficient for $\mathrm{n}$ - and p-type GaAs between 1.3 and $1.6 \mathrm{eV}$ " J. Appl. Phys., 46, 250 (1975).

[15] A. Chattopadhyay, S. Das Sarma, and A. J. Millis. "Transition Temperature of Ferromagnetic Semiconductors: A Dynamical Mean Field Study" Phys. Rev. Lett., 87, 227202 (2001).

[16] J. Chovan, E. G. Kavousanaki, and I. E. Perakis. "Ultrafast Light-Induced Magnetization Dynamics of Ferromagnetic Semiconductors" Phys. Rev. Lett., 96, 057402 (2006).

[17] J. Chovan and I. E. Perakis. "Femtosecond control of the magnetization in ferromagnetic semiconductors" Phys. Rev. B, 77, 085321 (2008).

[18] J. M. D. Coey, M. Venkatesan, and C. B. Fitzgerald. "Donor impurity band exchange in dilute ferromagnetic oxides" Nature Mat., 4, 173 (2005).

[19] S. Das Sarma, E. H. Hwang, and A. Kaminski. "Temperature-dependent magnetization in diluted magnetic semiconductors" Phys. Rev. B, 67, 155201 (2003).

[20] Y. Merle d'Aubigné, A. Arnoult, J. Cibert, T. Dietl, A. Haury, P. Kossacki, S. Tatarenko, and A. Wasiela. "Ferromagnetic transition induced by a twodimensional hole gas in semimagnetic quantum wells" Physica E, 3, 169 (1998).

[21] T. Dietl, H. Ohno, and F. Matsukura. "Hole-mediated ferromagnetism in tetrahedrally coordinated semiconductors" Phys. Rev. B, 63, 195205 (2001).

[22] T. Dietl, H. Ohno, F. Matsukura, J. Cibert, and D. Ferrand. "Zener Model Description 
of Ferromagnetism in Zinc-Blende Magnetic Semiconductors" Science, 287, 1019 (2000).

[23] K. W. Edmonds, P. Bogusławski, K. Y. Wang, R. P. Campion, S. N. Novikov, N. R. S. Farley, B. L. Gallagher, C. T. Foxon, M. Sawicki, T. Dietl, M. Buongiorno Nardelli, and J. Bernholc. "Mn Interstitial Diffusion in (Ga,Mn)As" Phys. Rev. Lett., 92, $037201(2004)$.

[24] S.A. Wolf et. al. "Spintronics: A Spin-Based Electronics Vision for the Future" Science, 294, 1488 (2001).

[25] M. Faraday. "Experimental researches in electricity" Phil. Trans. Roy. Soc., 136(1), 1846.

[26] Michael Farle. "Ferromagnetic resonance of ultrathin metallic layers" Rep. Prog. Phys., 61, 755 (1998).

[27] J. K. Furdyna. "Diluted magnetic semiconductors" J. Appl. Phys., 64, R29 (1988).

[28] P. Grünberg, R. Schreiber, Y. Pang, M. B. Brodsky, and H. Sowers. "Layered Magnetic Structures: Evidence for Antiferromagnetic Coupling of Fe Layers across Cr Interlayers" Phys. Rev. Lett., 57, 2442 (1986).

[29] Luca Guidoni, Eric Beaurepaire, and Jean-Yves Bigot. "Magneto-optics in the Ultrafast Regime: Thermalization of Spin Populations in Ferromagnetic Films" Phys. Rev. Lett., 89, 017401 (2002).

[30] K. Hamaya, T. Watanabe, T. Taniyama, A. Oiwa, Y. Kitamoto, and Y. Yamazaki. 
"Magnetic anisotropy switching in (Ga,Mn)As with increasing hole concentration" Phys. Rev. B, 74, 045201 (2006).

[31] Y. Hashimoto, S. Kobayashi, and H. Munekata. "Photoinduced Precession of Magnetization in Ferromagnetic (Ga,Mn)As" Phys. Rev. Lett., 100, 067202 (2008).

[32] A. Haury, A. Wasiela, A. Arnoult, J. Cibert, S. Tatarenko, T. Dietl, and Y. Merle d'Aubigné. "Observation of a Ferromagnetic Transition Induced by TwoDimensional Hole Gas in Modulation-Doped CdMnTe Quantum Wells" Phys. Rev. Lett., 79, 511 (1997).

[33] J. Hayakawa, S. Ikeda, Y.M. Lee, R. Sasaki, T. Meguro, F. Matsukura, and H. Ohno. "Current-Driven Magnetization Switching in $\mathrm{CoFeB} / \mathrm{MgO} / \mathrm{CoFeB}$ Magnetic Tunnel Junctions" Japan J. Appl. Phys., 44, 1267 (2005).

[34] T. Hibiya. "Growth and Characterization of Thick Liquid Phase Epitaxial Garnet Films and Their Magneto-optical Applications" Current Topics in Crystal Growth Research, 3, 199 (1997).

[35] Kimin Hong and N. Giordano. "Approach to mesoscopic magnetic measurements" Phys. Rev. B, 51, 9855 (1995).

[36] R. Huber, F. Tauser, A. Brodschelm, M. Bichler, G. Abstreiter, and A. Leitenstorfer. "How many-particle interactions develop after ultrafast excitation of an electron-hole plasma" Nature, 414, 286 (2001).

[37] Igor Žutić, Jaroslav Fabian, and S. Das Sarma. "Spintronics: Fundamentals and applications" Rev. Mod. Phys., 76, 323 (2004). 
[38] S. Ikeda, J. Hayakawa, Y.M. Lee, R. Sasaki, T. Meguro, F. Matsukura, and H. Ohno. "Dependence of Tunnel Magnetoresistance in MgO Based Magnetic Tunnel Junctions on Ar Pressure during MgO Sputtering” Japan J. Appl. Phys., 44, 1442 (2005).

[39] Y. Ishiwata, T. Takeuchi, R. Eguchi, M. Watanabe, Y. Harada, K. Kanai, A. Chainani, M. Taguchi, S. Shin, M. C. Debnath, I. Souma, Y. Oka, T. Hayashi, Y. Hashimoto, S. Katsumoto, and Y. Iye. "Direct observation of a neutral Mn acceptor in $\mathrm{Ga}_{1-x} \mathrm{Mn}_{x}$ As by resonant x-ray emission spectroscopy" Phys. Rev. B, 71, 121202 (2005).

[40] T. Jungwirth, Jairo Sinova, J. Masek, J. Kucera, and A. H. MacDonald. "Theory of ferromagnetic (III,Mn)V semiconductors" Rev. Mod. Phys., 78, 809 (2006).

[41] T. Jungwirth, K. Y. Wang, J. Mašek, K. W. Edmonds, Jürgen König, Jairo Sinova, M. Polini, N. A. Goncharuk, A. H. MacDonald, M. Sawicki, A. W. Rushforth, R. P. Campion, L. X. Zhao, C. T. Foxon, and B. L. Gallagher. "Prospects for high temperature ferromagnetism in (Ga,Mn)As semiconductors" Phys. Rev. B, 72, 165204 $(2005)$.

[42] Tadao Kasuya. "A Theory of Metallic Ferro- and Antiferromagnetism on Zener's Model" Prog. Theor. Phys., 16, 45 (1956).

[43] A. V. Kimel, G. V. Astakhov, A. Kirilyuk, G. M. Schott, G. Karczewski, W. Ossau, G. Schmidt, L. W. Molenkamp, and Th. Rasing. "Observation of Giant Magnetic Linear Dichroism in (Ga,Mn)As" Phys. Rev. Lett., 94, 227203 (2005).

[44] E. Kojima, R. Shimano, Y. Hashimoto, S. Katsumoto, Y. Iye, and M. Kuwata- 
Gonokami. "Observation of the spin-charge thermal isolation of ferromagnetic $\mathrm{Ga}_{0.94} \mathrm{Mn}_{0.06} \mathrm{As}$ by time-resolved magneto-optical measurements" Phys. Rev. B, 68, $193203(2003)$.

[45] B. Koopmans, M. van Kampen, J. T. Kohlhepp, and W. J. M. de Jonge. "Ultrafast Magneto-Optics in Nickel: Magnetism or Optics?" Phys. Rev. Lett., 85, 844 (2000).

[46] P. A. Korzhavyi, I. A. Abrikosov, E. A. Smirnova, L. Bergqvist, P. Mohn, R. Mathieu, P. Svedlindh, J. Sadowski, E. I. Isaev, Yu. Kh. Vekilov, and O. Eriksson. "DefectInduced Magnetic Structure in $\mathrm{Ga}_{1-x} \mathrm{Mn}_{x} \mathrm{As}$ " Phys. Rev. Lett., 88, 187202 (2002).

[47] S. Koshihara, A. Oiwa, M. Hirasawa, S. Katsumoto, Y. Iye, C. Urano, H. Takagi, and H. Munekata. "Ferromagnetic Order Induced by Photogenerated Carriers in Magnetic III-V Semiconductor Heterostructures of (In,Mn)As/GaSb” Phys. Rev. Lett., 78, 4617 (1997).

[48] K. C. Ku, S. J. Potashnik, R. F. Wang, S. H. Chun, P. Schiffer, N. Samarth, M. J. Seong, A. Mascarenhas, E. Johnston-Halperin, R. C. Myers, A. C. Gossard, and D. D. Awschalom. "Highly enhanced Curie temperature in low-temperature annealed [Ga,Mn]As epilayers" Appl. Phys. Lett., 82, 2302 (2003).

[49] M. Linnarsson, E. Janzén, B. Monemar, M. Kleverman, and A. Thilderkvist. "Electronic structure of the GaAs: $\mathrm{Mn}_{G a}$ center" Phys. Rev. B, 55, 6938 (1997).

[50] X. Liu, W. L. Lim, M. Dobrowolska, J. K. Furdyna, and T. Wojtowicz. "Ferromagnetic resonance study of the free-hole contribution to magnetization and magnetic 
anisotropy in modulation-doped GaMnAs/GaAlAs:Be" Phys. Rev. B., 71, 035307 (2005).

[51] X. Liu, W. L. Lim, L. V. Titova, M. Dobrowolska, J. K. Furdyna, M. Kutrowski, and T. Wojtowicz. "Perpendicular magnetization reversal, magnetic anisotropy, multistep spin switching, and domain nucleation and expansion in $\mathrm{Ga}_{1-x} \mathrm{Mn}_{x}$ As films" J. Appl. Phys., 98, 063904 (2005).

[52] X. Liu, Y. Sasaki, and J. K. Furdyna. "Ferromagnetic resonance in $\mathrm{Ga}_{1-x} \mathrm{Mn}_{x} \mathrm{As}$ : Effects of magnetic anisotropy" Phys. Rev. B, 67, 205204 (2003).

[53] A. H. MacDonald, P. Schiffer, and N. Samarth. "Ferromagnetic semiconductors: moving beyond (Ga,Mn)As" Nature Mat., 4, 195 (2005).

[54] Priya Mahadevan, Alex Zunger, and D. D. Sarma. "Unusual Directional Dependence of Exchange Energies in GaAs Diluted with Mn: Is the RKKY Description Relevant?" Phys. Rev. Lett., 93, 177201 (2004).

[55] F. Matsukura, H. Ohno, A. Shen, and Y. Sugawara. "Transport properties and origin of ferromagnetism in (Ga,Mn)As" Phys. Rev. B, 57, R2037 (1998).

[56] A. Mikkelsen, J. Gustafson, J. Sadowski, J.N. Andersen, J. Kanski, and E. Lundgren. "A study of the surface structure and composition of annealed $\mathrm{Ga}_{0.96} \mathrm{Mn}_{0.04} \mathrm{As}$ (100)" Appl. Surf. Sci., 222, 23 (2004).

[57] H. Munekata, H. Ohno, S. von Molnar, Armin Segmüller, L. L. Chang, and L. Esaki. "Diluted magnetic III-V semiconductors" Phys. Rev. Lett., 63, 1849 (1989). 
[58] Ahsan M. Nazmul, S. Sugahara, and M. Tanaka. "Ferromagnetism and high Curie temperature in semiconductor heterostructures with $\mathrm{Mn} \delta$-doped GaAs and p-type selective doping” Phys. Rev. B, 67, 241308 (2003).

[59] S. B. Ogale, R. J. Choudhary, J. P. Buban, S. E. Lofland, S. R. Shinde, S. N. Kale, V. N. Kulkarni, J. Higgins, C. Lanci, J. R. Simpson, N. D. Browning, S. Das Sarma, H. D. Drew, R. L. Greene, and T. Venkatesan. "High Temperature Ferromagnetism with a Giant Magnetic Moment in Transparent Co-doped $\mathrm{SnO}_{2-\delta}$ " Phys. Rev. Lett., 91, 077205 (2003).

[60] H. Ohno. "Making Nonmagnetic Semiconductors Ferromagnetic" Science, 281, 951956 (1998).

[61] H. Ohno, D. Chiba, F. Matsukura, T. Omiya, E. Abe, T. Dietl, Y. Ohno, and K. Ohtani. "Electric-field control of ferromagnetism" Nature, 408, 944 (2000).

[62] H. Ohno, F. Matsukura, and Y. Ohno. "Spintronics: A Spin-Based Electronics Vision for the Future" JSAP International, 5, 4 (2002).

[63] H. Ohno, A. Shen, F. Matsukura, A. Oiwa, A. Endo, S. Katsumoto, and Y. Iye. "(Ga,Mn)As: A new diluted magnetic semiconductor based on GaAs" Appl. Phys. Lett., 69, 363 (1996).

[64] Hideo Ohno. "Properties of ferromagnetic III-V semiconductors" J. Mag. Mag. Mat., 200, 110 (1999).

[65] Stuart S. P. Parkin, Christian Kaiser, Alex Panchula, Philip M. Rice, Brian Hughes, 
Mahesh Samant, and See-Hun Yang. "Giant tunnelling magnetoresistance at room temperature with $\mathrm{MgO}$ (100) tunnel barriers" Nature Mat., 3, 862 (2004).

[66] J. Qi, Y. Xu, N. H. Tolk, X. Liu, J. K. Furdyna, and I. E. Perakis. "Coherent magnetization precession in GaMnAs induced by ultrafast optical excitation" Appl. Phys. Lett., 91, 112506 (2007).

[67] H. Regensburger, R. Vollmer, and J. Kirschner. "Time-resolved magnetizationinduced second-harmonic generation from the Ni(110) surface" Phys. Rev. B, 61, $14716(2000)$.

[68] K. Rode, A. Anane, R. Mattana, J.-P. Contour, O. Durand, and R. LeBourgeois. "Magnetic semiconductors based on cobalt substituted ZnO" J. Appl. Phys., 93, $7676(2003)$.

[69] M. A. Ruderman and C. Kittel. "Indirect Exchange Coupling of Nuclear Magnetic Moments by Conduction Electrons" Phys. Rev., 96, 99 (1954).

[70] Hiromasa Saeki, Hitoshi Tabata, and Tomoji Kawai. "Magnetic and electric properties of vanadium doped ZnO films" Solid State Commun., 120, 439 (2001).

[71] H. Saito, V. Zayets, S. Yamagata, and K. Ando. "Room-Temperature Ferromagnetism in a II-VI Diluted Magnetic Semiconductor $\mathrm{Zn}_{1-x} \mathrm{Cr}_{x}$ Te" Phys. Rev. Lett., 90, 207202 (2003).

[72] V. F. Sapega, M. Moreno, M. Ramsteiner, L. Däweritz, and K. H. Ploog. "Polarization of Valence Band Holes in the (Ga,Mn)As Diluted Magnetic Semiconductor" Phys. Rev. Lett., 94, 137401 (2005). 
[73] Y. Satoh, N. Inoue, Y. Nishikawa, and J. Yoshino. "Proceedings of the $3^{\text {rd }}$ Symposium on Physics and Application of Spin-Related Phenomena in Semiconductors" pages $23-25$ (1997).

[74] M. A. Scarpulla, B. L. Cardozo, R. Farshchi, W. M. Hlaing Oo, M. D. McCluskey, K. M. Yu, and O. D. Dubon. "Ferromagnetism in $\mathrm{Ga}_{1-x} \mathrm{Mn}_{x} \mathrm{P}$ : Evidence for InterMn Exchange Mediated by Localized Holes within a Detached Impurity Band” Phys. Rev. Lett., 95, 207204 (2005).

[75] M. A. Scarpulla, O. D. Dubon, K. M. Yu, O. Monteiro, M. R. Pillai, and M. J. Aziz. "Ferromagnetic $\mathrm{Ga}_{1-x} \mathrm{Mn}_{x}$ As produced by ion implantation and pulsed-laser melting" Appl. Phys. Lett., 82, 1251 (2003).

[76] G. M. Schott, W. Faschinger, and L. W. Molenkamp. "Lattice constant variation and complex formation in zincblende gallium manganese arsenide" Appl. Phys. Lett., 79, 1807 (2001).

[77] A. Shen, H. Ohno, F. Matsukura, Y. Sugawara, N. Akiba, T. Kuroiwa, A. Oiwa, A. Endo, S. Katsumoto, and Y. Iye. "Epitaxy of (Ga, Mn)As, a new diluted magnetic semiconductor based on GaAs" J. Cryst. Growth, 175/176, 1069 (1997).

[78] Noriyoshi Shibata, Akira Ohki, and Akinori Katsui. "Iodine-doped low-resistivity n-type ZnSe films grown by MOVPE” J.Cryst. Growth, 93, 703 (1988).

[79] Avinash Singh, Animesh Datta, Subrat K. Das, and Vijay A. Singh. "Ferromagnetism in a dilute magnetic semiconductor: Generalized RKKY interaction and spin-wave excitations" Phys. Rev. B, 68, 235208 (2003). 
[80] I. P. Smorchkova, N. Samarth, J. M. Kikkawa, and D. D. Awschalom. "Spin Transport and Localization in a Magnetic Two-Dimensional Electron Gas" Phys. Rev. Lett., 78, 3571 (1997).

[81] Saki Sonoda, Saburo Shimizu, Takahiko Sasaki, Yoshiyuki Yamamoto, and Hidenobu Hori. "Molecular beam epitaxy of wurtzite $(\mathrm{Ga}, \mathrm{Mn}) \mathrm{N}$ films on sapphire( $\left.\begin{array}{llll}0 & 0 & 0 & 1\end{array}\right)$ showing the ferromagnetic behaviour at room temperature" J. Cryst. Growth, 237, 1358 (2002).

[82] B. S. Sorensen, P. E. Lindelof, J. Sadowski, R. Mathieu, and P. Svedlindh. "Effect of annealing on carrier density and Curie temperature in epitaxial (Ga,Mn)As thin films" Appl. Phys. Lett., 82, 2287 (2003).

[83] P. R. Stone, M. A. Scarpulla, R. Farshchi, I. D. Sharp, E. E. Haller, O. D. Dubon, K. M. Yu, J. W. Beeman, E. Arenholz, and J. D. Denlinger. "Mn $\mathrm{L}_{3,2}$ x-ray absorption and magnetic circular dichroism in ferromagnetic $\mathrm{Ga}_{1-x} \mathrm{Mn}_{x} \mathrm{P} "$ Appl. Phys. Lett., 89, 012504 (2006).

[84] T. Story, R. R. Galazka, R. B. Frankel, and P. A. Wolff. "Carrier-concentrationinduced ferromagnetism in PbSnMnTe" Phys. Rev. Lett., 56, 777 (1986).

[85] M. D. Sturge. "Optical Absorption of Gallium Arsenide between 0.6 and 2.75 eV" Phys. Rev., 127, 768 (1962).

[86] S. Sugano and N. Kojima. "Magneto-Optics" Springer, 2000.

[87] H. X. Tang, R. K. Kawakami, D. D. Awschalom, and M. L. Roukes. "Giant Planar Hall Effect in Epitaxial (Ga,Mn)As Devices" Phys. Rev. Lett., 90, 107201 (2003). 
[88] F. Tuomisto, K. Pennanen, K. Saarinen, and J. Sadowski. "Ga Sublattice Defects in (Ga,Mn)As: Thermodynamical and Kinetic Trends" Phys. Rev. Lett., 93, 055505 (2004).

[89] W. G. van der Wiel, S. De Franceschi, J. M. Elzerman, T. Fujisawa, S. Tarucha, and L. P. Kouwenhoven. "Electron transport through double quantum dots" Rev. Mod. Phys., 75, 1 (2002).

[90] M. van Kampen, C. Jozsa, J. T. Kohlhepp, P. LeClair, L. Lagae, W. J. M. de Jonge, and B. Koopmans. "All-Optical Probe of Coherent Spin Waves" Phys. Rev. Lett., 88, $227201(2002)$.

[91] S. von Molnar, H. Munekata, H. Ohno, and L.L. Chang. "New diluted magnetic semiconductors based on III-V compounds" J. Magn. Magn. Mater., 93, 356 (1991).

[92] D. M. Wang, Y. H. Ren, X. Liu, J. K. Furdyna, M. Grimsditch, and R. Merlin. "Lightinduced magnetic precession in (Ga,Mn)As slabs: Hybrid standing-wave DamonEshbach modes" Phys. Rev. B, 75, 233308 (2007).

[93] J. Wang, I. Cotoros, D. S. Chemla, X. Liu, J. K. Furdyna, J. Chovan, and I. E. Perakis. "Memory Effect in Photoinduced Femtosecond Magnetization Rotation in GaMnAs" Appl. Phys. Lett., 93, TBD (2008).

[94] J. Wang, I. Cotoros, K. M. Dani, X. Liu, J. K. Furdyna, and D. S. Chemla. "Ultrafast Enhancement of Ferromagnetism via Photoexcited Holes in GaMnAs" Phys. Rev. Lett., 98, 217401 (2007). 
[95] J. Wang, Y. Hashimoto, J. Kono, A. Oiwa, H. Munekata, G. D. Sanders, and C. J. Stanton. "Propagating coherent acoustic phonon wave packets in $\operatorname{In}_{x} \mathrm{Mn}_{1-x} \mathrm{As} / \mathrm{GaSb}$ " Phys. Rev. B, 72, 153311 (2005).

[96] J. Wang, C. Sun, Y. Hashimoto, J. Kono, G. A. Khodaparast, L. Cywinski, L. J. Sham, G. D. Sanders, C. J. Stanton, and H. Munekata. "Ultrafast magneto-optics in ferromagnetic III-V semiconductors" J. Phys.: Condens. Matter., 18, R501 (2006).

[97] J. Wang, C. Sun, J. Kono, A. Oiwa, H. Munekata, Ł. Cywiński, and L. J. Sham. "Ultrafast Quenching of Ferromagnetism in InMnAs Induced by Intense Laser Irradiation" Phys. Rev. Lett., 95, 167401 (2005).

[98] U. Welp, V. K. Vlasko-Vlasov, X. Liu, J. K. Furdyna, and T. Wojtowicz. "Magnetic Domain Structure and Magnetic Anisotropy in $\mathrm{Ga}_{1-x} \mathrm{Mn}_{x} \mathrm{As}$ " Phys. Rev. Lett., 90, 167206 (2003).

[99] P. E. Wigen. "Dipole-Narrowed Inhomogeneously Broadened Lines in Ferromagnetic Thin Films" Phys. Rev., 133, A1557 (1964).

[100] S. A. Wolf, D. D. Awschalom, R. A. Buhrman, J. M. Daughton, S. von Molnar, M. L. Roukes, A. Y. Chtchelkanova, and D. M. Treger. "Spintronics: A Spin-Based Electronics Vision for the Future" Science, 294, 1488 (2001).

[101] Stephen Y. Wu, H. X. Liu, Lin Gu, R. K. Singh, L. Budd, M. van Schilfgaarde, M. R. McCartney, David J. Smith, and N. Newman. "Synthesis, characterization, and modeling of high quality ferromagnetic Cr-doped AlN thin films" Appl. Phys. Lett., 82, 3047 (2003). 
[102] Kei Yosida. "Magnetic Properties of Cu-Mn Alloys" Phys. Rev., 106, 893 (1957).

[103] C. Y. You and S. C. Shin. "Derivation of simplified analytic formulae for magnetooptical Kerr effects" Appl. Phys. Lett., 69, 1315 (1996).

[104] K. M. Yu, W. Walukiewicz, T. Wojtowicz, I. Kuryliszyn, X. Liu, Y. Sasaki, and J. K. Furdyna. "Effect of the location of Mn sites in ferromagnetic $\mathrm{Ga}_{1-x} \mathrm{Mn}_{x} \mathrm{As}$ on its Curie temperature" Phys. Rev. B, 65, 201303 (2002).

[105] Shinji Yuasa, Taro Nagahama, Akio Fukushima, Yoshishige Suzuki, and Koji Ando. "Giant room-temperature magnetoresistance in single-crystal $\mathrm{Fe} / \mathrm{MgO} / \mathrm{Fe}$ magnetic tunnel junctions" Nature Mat., 3, 868 (2004).

[106] J. M. Ziman. "Principles of the Theory of Solids" Cambridge University Press, 1999. 